\title{
Methods in perioperative medicine research
}
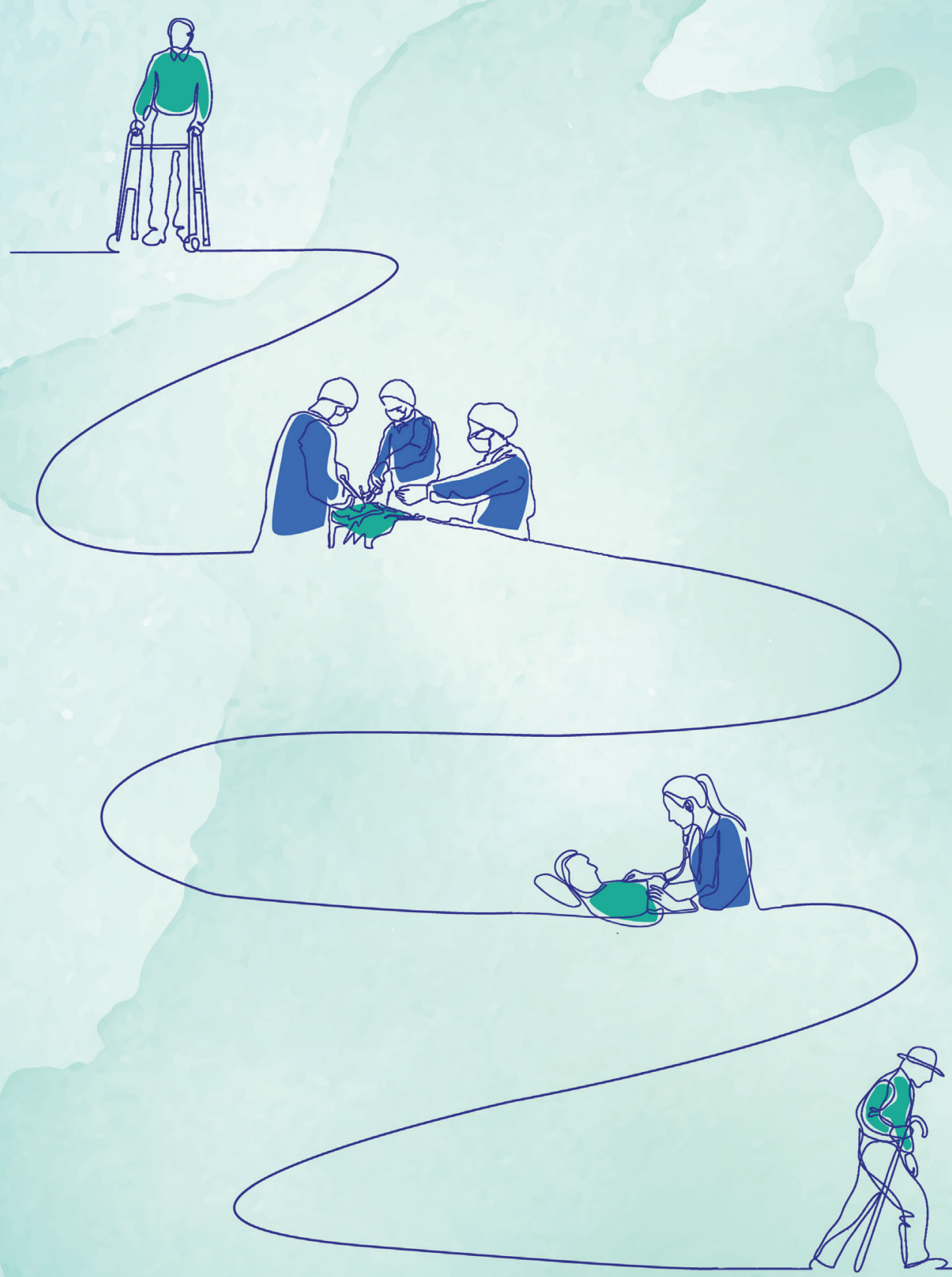

Lisette Vernooij 


\section{Methods in Perioperative Medicine Research}

Lisette Margaretha Vernooij 


\section{Colofon}

Methods in Perioperative Medicine Research

ISBN/EAN: 978-94-6375-882-6

Copyright $\odot 2020$ Lisette Vernooij

All rights reserved. No part of this thesis may be reproduced, stored or transmitted in any way or by any means without the prior permission of the author, or when applicable, of the publishers of the scientific papers.

Cover design and Layout by Birgit Vredenburg | persoonlijkproefschrift.nl.

Printed by Ridderprint | www.ridderprint.nl

Financial support by the department of Anesthesiology of the UMC Utrecht and Dutch Heart Foundation for the publication of this thesis is gratefully acknowledged. 
Methods in Perioperative Medicine Research

\section{Onderzoeksmethodologie in de perioperatieve periode}

(met een samenvatting in het Nederlands)

\section{Proefschrift}

ter verkrijging van de graad van doctor aan de

Universiteit Utrecht

op gezag van de

rector magnificus, prof.dr. H.R.B.M. Kummeling,

ingevolge het besluit van het college voor promoties

in het openbaar te verdedigen op

dinsdag 29 september 2020 des ochtends te 9.15 uur

door

\section{Lisette Margaretha Vernooij}

geboren op 23 mei 1991

te Bunnik 


\section{PROMOTOREN:}

Prof. dr. W.A. van Klei

Prof. dr. K.G.M. Moons

COPROMOTOREN:

Dr. L.M. Slingerland-Peelen

Dr. J.A.R. van Waes 


\section{TABLE OF CONTENTS}

\section{PART 1 INTRAOPERATIVE HYPOTENSION}

Chapter 2

Methods to express intraoperative hypotension exposure in anaesthesia literature

Different methods of modelling intraoperative hypotension and

Chapter 3 their association with postoperative complications in patients undergoing non-cardiac surgery

\section{PART 2 ADDED VALUE OF BIOMARKERS TO THE RCRI}

The added value of biomarkers to the Revised Cardiac Risk Index

Chapter 4 to predict major adverse cardiac events and all-cause mortality after non-cardiac surgery: protocol of a systematic review and meta-analysis

The added value of biomarkers to the Revised Cardiac Risk

Chapter 5 Index to preoperatively predict major adverse cardiac events in patients who undergo non-cardiac surgery: a systematic review

\section{PART 3 TROPONIN ELEVATION AFTER MAJOR NON-CARDIAC SURGERY}

Postoperative visits by dedicated anaesthesiologists in patients

Chapter 6

Chapter 7 with elevated troponin: a retrospective cohort study evaluating postoperative care utility and early detection of complications

Association between postoperative myocardial injury phenotypes and disability-free survival in patients undergoing non-cardiac surgery

Chapter 8

General discussion

Appendices

Publication list

Dankwoord 


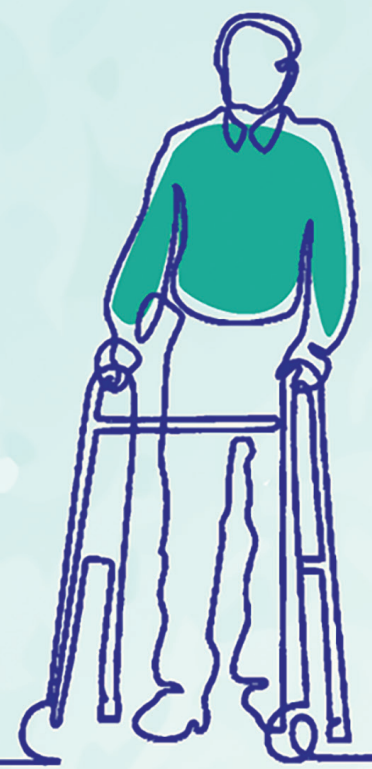




\section{CHAPTER}

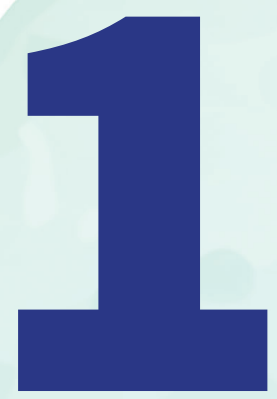

General introduction 
Worldwide, over 320 million patients undergo non-cardiac surgery every year and this number is increasing continuously. $(1,2)$ Obviously, the ultimate goal of surgery is to improve quality of life and to maintain or increase life expectancy by curing disease or relieving symptoms. However, surgery is not without risks and approximately one out of six patients undergoing surgery will develop a postoperative complication. (3) Infectious complications occur most frequently (33\%), followed by cardiovascular complications (19\%). (3) Cardiovascular complications are a leading cause of postoperative morbidity and mortality (4-7) and are associated with prolonged hospitalization and increased medical costs. (8) Preoperative risk stratification and early identification of patients at risk for such complications is therefore important to direct care towards patients who need it the most in order to prevent such adverse events and possibly even death.

\section{Perioperative cardiac complications}

As a result of the physiological stress response to surgery, patients experience sympathetic hyperactivity, which is characterized by an increased release of plasma catecholamines. This physiological stress test in turn may result in hemodynamic instability and coronary vasoconstriction. $(9,10)$ These conditions occur commonly in the perioperative phase and can lead to myocardial ischemia due an imbalance of oxygen supply and demand. $(9,10)$ Postoperative myocardial injury (PMI) after non-cardiac surgery occurs in approximately 11-19\% of patients, depending on the study population and emergency status of the surgery. $(4-7,11) \mathrm{PMI}$ is defined as an elevated serum troponin value above the 99th percentile of the upper reference limit. (12) Troponin is a protein that is involved in the contraction of cardiac muscle and is released by injured cardiomyocytes. Release of troponin may be due to myocardial cell death caused by ischemia, but also by normal turnover of myocardial cells, apoptosis or increased permeability of the cell membrane. (13) PMI is an independent predictor for morbidity and mortality, in a concentrationdependent manner. $(4-7,11)$

The most common phenotype of PMI is myocardial infarction. Postoperative myocardial infarction occurs in 3-5\% of patients undergoing major non-cardiac surgery. (4-7) Myocardial infarction is defined as myocardial cell death due to prolonged ischemia. (12) It is diagnosed as acute myocardial injury with clinical evidence of acute myocardial ischemia and with detection of a rise and/or fall of cardiac enzymes with at least one value above the 99th percentile upper reference limit. In addition, patients must present with at least one of the following: symptoms of myocardial ischemia, new ischemic ECG changes, development of pathological Q waves, imaging evidence of new loss of viable myocardium or new regional wall motion abnormality in a pattern consistent with an ischemic aetiology. (12) However, recognition of myocardial infarction in the perioperative phase is difficult because most patients do not experience typical symptoms. $(6,14)$ Chest pain may for example be masked by the analgesic and sedative effects of painkillers and anaesthetics, and distracting pain from the surgical wound. Other symptoms, such as dyspnoea, nausea and 
fatigue could be interpreted as other common postoperative problems, such as pneumonia, hypovolemia or medication side effects. (15) Consequently, myocardial injury and infarction are commonly not recognized and hence not treated although patients experiencing these conditions are known to have an increased risk of mortality. $(4-7,16)$

\section{Preoperative risk stratification}

In the ideal world, a treating physician knows before surgery which patients will develop complications. As clinical judgement based on intuition is often inaccurate and inconsistent, (17) prediction models can be used to determine an individual's risk profile. Such models may inform patients and their treating physicians on the probability of a certain prognostic outcome, in this case postoperative cardiac complications. This information could be useful in making decisions in the treatment of individual patients. (18) Several prognostic models have been developed that aim to preoperatively predict postoperative cardiac complications. (19-21) Nowadays, the Revised Cardiac Risk Index (RCRI) (19) is the most commonly used risk model in clinical practice and has been recommended by guidelines. $(10,22)$ The RCRI contains six equally weighted predictors, i.e. high risk surgery, history of ischemic heart disease, history of congestive heart failure, history of cerebrovascular disease, preoperative elevated creatinine level and insulin dependent diabetes. (19) The model is easy to use as the predictors are easy to collect and calculation of the score is convenient. External validation of the RCRI showed overall moderate predictive performance, however predictions for patients undergoing vascular surgery were less accurate. (23) Several studies added biomarkers to the RCRI in an attempt to improve the prediction of postoperative cardiac complications. (24-26) Others compared the predictive performance of single biomarkers to the RCRI. $(27,28)$ However, there is currently no comprehensive overview available of all biomarkers that have been used to improve risk prediction of postoperative cardiac complications.

\section{Intraoperative hypotension}

Besides preoperative factors, also intraoperative factors have been identified to be associated with adverse postoperative cardiac events. The most commonly studied intraoperative factor is hypotension. Hypotension refers to a blood pressure that is too low. As mentioned before, it has been thought that hemodynamic instability including hypotension contributes to an ischemic disbalance of the myocardium by causing a decreased oxygen supply. (12) Intraoperative hypotension has been related to ischemia-reperfusion injury of multiple organs including the heart, brain and kidneys and is associated with mortality. (29-32) As intraoperative hypotension is common during surgery and represents a potential modifiable factor, this topic has been increasingly investigated. (31) 
In the last decade, much debate has been going on regarding the definition of intraoperative hypotension. In other words: "what blood pressure is too low?" Bijker and colleagues found 140 definitions of $\mathrm{IOH}$ in 130 articles with incidences of hypotension ranging from 5 to 95\%. (33) As a result, a consensus statement on the definition of hypotension has been published recently, which says that maintaining a systolic arterial pressure above $100 \mathrm{~mm} \mathrm{Hg}$ and a mean arterial pressure above 60-70 mm Hg may reduce the risk of adverse outcomes, including myocardial injury, acute kidney injury, and death. (34)

\section{Postoperative routine troponin monitoring}

Because preoperative identification of patients at risk has proven to be difficult, one strategy to identify patients at high risk for complications is through troponin monitoring in the early postoperative phase, which has been advocated in several guidelines. (10, 12, 22) As mentioned before, troponin elevation, or PMI, is associated with poor prognosis. $(4-7,11)$ PMI has been related to a spectrum of different phenotypes ranging from no injury to myocardial infarction. (12) Myocardial infarction is the best known phenotype of PMI. However, besides myocardial infarction that is attributed to myocardial ischemia, other PMI phenotypes include arrhythmia (most often atrial fibrillation) and heart failure. Furthermore, non-cardiac adverse events such as pulmonary embolism, sepsis, respiratory failure and acute kidney injury have also been related to PMI. $(11,12,35,36)$ As a result, the University Medical Center Utrecht implemented a local protocol to routinely measure troponin in all patients above 60 years undergoing intermediate to high-risk surgery in the first three postoperative days. A dedicated anaesthesiologist visits all patients with PMI to enhance recognition of myocardial infarction and other complications related to $\mathrm{PMI}$ at an early stage.

\section{Thesis objective}

The objective of this thesis is to investigate the prediction and management of in-hospital cardiac events and complications and their influence on long term outcomes in patients undergoing non-cardiac surgery.

\section{Outline of this thesis}

Intraoperative hypotension has been associated with cardiac complications. However, summarizing repeated measured blood pressure measures remains challenging for research purposes. Part I focuses on different methodologies on how to incorporate intraoperative hypotension and its severity in statistical models. Chapter $\mathbf{2}$ describes what methods to express intraoperative hypotension have been used in recent anaesthesia literature. Chapter $\mathbf{3}$ reports on the association between twelve different methods to model the severity of intraoperative hypotension and postoperative myocardial injury in non-cardiac surgery patients. 
Preoperative risk prediction is an important strategy to identify patients at high risk for postoperative complications. As predictions of the RCRI are only moderate, Part II (Chapters 4 and 5) provides an overview what biomarkers have been added to the RCRI or compared its predictive performance to improve risk prediction. Chapter $\mathbf{4}$ describes the protocol of a systematic review on the added predictive value of biomarker(s) to the RCRI and to estimate the predictive performance of biomarkers itself compared to the RCRI to predict postoperative outcomes in patients undergoing non-cardiac surgery. Chapter 5 represents the full Cochrane systematic review.

Next, Part III addresses management and long term effects of patients with postoperative troponin elevation. The implementation of follow-up visits by perioperative anaesthesiologists in patients with postoperative troponin elevation during hospital admission was evaluated in

Chapter $\mathbf{6}$. The distinction of different PMI phenotypes in association with long term disability free survival was investigated in Chapter $\mathbf{7}$.

Finally, this thesis ends with a general discussion, which summarizes the main findings, discusses the previous chapters in a broader context and considers future research possibilities. 


\section{REFERENCES}

1. Rose J, Weiser TG, Hider P, Wilson L, Gruen RL, Bickler SW. Estimated need for surgery worldwide based on prevalence of diseases: a modelling strategy for the WHO Global Health Estimate. Lancet Glob Health. 2015;3 Suppl 2:S13-20.

2. Weiser TG, Haynes AB, Molina G, Lipsitz SR, Esquivel MM, Uribe-Leitz T, et al. Estimate of the global volume of surgery in 2012: an assessment supporting improved health outcomes. Lancet. 2015;385 Suppl 2:S11.

3. Global patient outcomes after elective surgery: prospective cohort study in 27 low-, middleand high-income countries. Br J Anaesth. 2016;117(5):601-9.

4. van Waes JAR, Nathoe HM, de Graaff JC, Kemperman H, de Borst GJ, Peelen LM, et al. Myocardial Injury After Noncardiac Surgery and its Association With Short-Term Mortality. Circulation. 2013;127(23):2264-71.

5. Beattie WS, Karkouti K, Tait G, Steel A, Yip P, McCluskey S, et al. Use of clinically based troponin underestimates the cardiac injury in non-cardiac surgery: a single-centre cohort study in 51,701 consecutive patients. Can J Anaesth. 2012;59(11):1013-22.

6. Devereaux PJ, Biccard BM, Sigamani A, Xavier D, Chan MTV, Srinathan SK, et al. Association of Postoperative High-Sensitivity Troponin Levels With Myocardial Injury and 30-Day Mortality Among Patients Undergoing Noncardiac Surgery. Jama. 2017;317(16):1642-51.

7. Devereaux PJ, Chan MTV, Alonso-Coello P, Walsh M, Berwanger O, Villar JC, et al. Association between postoperative troponin levels and 30-day mortality among patients undergoing noncardiac surgery. JAMA - Journal of the American Medical Association. 2012;307(21):2295304.

8. Mackey WC, Fleisher LA, Haider S, Sheikh S, Cappelleri JC, Lee WC, et al. Perioperative myocardial ischemic injury in high-risk vascular surgery patients: incidence and clinical significance in a prospective clinical trial. J Vasc Surg. 2006;43(3):533-8.

9. Landesberg G, Beattie WS, Mosseri M, Jaffe AS, Alpert JS. Perioperative Myocardial Infarction. Circulation. 2009;119(22):2936-44.

10. Kristensen SD, Knuuti J, Saraste A, Anker S, Botker HE, De Hert S, et al. 2014 ESC/ESA Guidelines on non-cardiac surgery: cardiovascular assessment and management The Joint Task Force on non-cardiac surgery: cardiovascular assessment and management of the European Society of Cardiology (ESC) and the European Society of Anaesthesiology (ESA). European Heart Journal. 2014;35(35):2383-431.

11. van Waes JAR, Grobben RB, Nathoe HM, Kemperman H, de Borst GJ, Peelen LM, et al. OneYear Mortality, Causes of Death, and Cardiac Interventions in Patients with Postoperative Myocardial Injury. Anesthesia and Analgesia. 2016;123(1):29-37.

12. Thygesen K, Alpert JS, Jaffe AS, Chaitman BR, Bax JJ, Morrow DA, et al. Fourth Universal Definition of Myocardial Infarction (2018). Circulation. 2018;138(20):e618-e51.

13. Mair J, Lindahl B, Hammarsten O, Muller C, Giannitsis E, Huber K, et al. How is cardiac troponin released from injured myocardium? Eur Heart J Acute Cardiovasc Care. 2018;7(6):553-60.

14. Puelacher C, Buse GL, Seeberger D, Sazgary L, Marbot S, Lampart A, et al. Perioperative Myocardial Injury After Noncardiac Surgery Incidence, Mortality, and Characterization. Circulation. 2018;137(12):1221-32. 
15. Devereaux PJ, Goldman L, Cook DJ, Gilbert K, Leslie K, Guyatt GH. Perioperative cardiac events in patients undergoing noncardiac surgery: a review of the magnitude of the problem, the pathophysiology of the events and methods to estimate and communicate risk. Canadian Medical Association Journal. 2005;173(6):627-34.

16. Beattie WS, Wijeysundera DN, Chan MTV, Peyton PJ, Leslie K, Paech MJ, et al. Survival After Isolated Post-Operative Troponin Elevation. J Am Coll Cardiol. 2017;70(7):907-8.

17. Hoffmann TC, Del Mar C. Clinicians' Expectations of the Benefits and Harms of Treatments, Screening, and Tests: A Systematic Review. JAMA Intern Med. 2017;177(3):407-19.

18. Steyerberg EW. Clinical Prediction Models: A Practical Approach to Development, Validation, and Updating: Springer New York; 2008.

19. Lee TH, Marcantonio ER, Mangione CM, Thomas EJ, Polanczyk CA, Cook EF, et al. Derivation and prospective validation of a simple index for prediction of cardiac risk of major noncardiac surgery. Circulation. 1999;100(10):1043-9.

20. Cohen ME, Ko CY, Bilimoria KY, Zhou L, Huffman K, Wang X, et al. Optimizing ACS NSQIP modeling for evaluation of surgical quality and risk: patient risk adjustment, procedure mix adjustment, shrinkage adjustment, and surgical focus. J Am Coll Surg. 2013;217(2):336-46. e1.

21. Gupta PK, Gupta H, Sundaram A, Kaushik M, Fang X, Miller WJ, et al. Development and Validation of a Risk Calculator for Prediction of Cardiac Risk After Surgery. Circulation. 2011;124(4):381-U146.

22. Fleisher LA, Fleischmann KE, Auerbach AD, Barnason SA, Beckman JA, Bozkurt B, et al. 2014 ACC/AHA Guideline on Perioperative Cardiovascular Evaluation and Management of Patients Undergoing Noncardiac Surgery: Executive Summary A Report of the American College of Cardiology/American Heart Association Task Force on Practice Guidelines. Circulation. 2014;130(24):2215-45.

23. Ford MK, Beattie WS, Wijeysundera DN. Systematic Review: Prediction of Perioperative Cardiac Complications and Mortality by the Revised Cardiac Risk Index. Annals of Internal Medicine. 2010;152(1):26-W7.

24. Choi JH, Cho DK, Song YB, Hahn JY, Choi S, Gwon HC, et al. Preoperative NT-proBNP and CRP predict perioperative major cardiovascular events in non-cardiac surgery. Heart. 2010;96(1):56-62.

25. Kopec M, Duma A, Helwani MA, Brown J, Brown F, Gage BF, et al. Improving Prediction of Postoperative Myocardial Infarction With High-Sensitivity Cardiac Troponin T and NTproBNP. Anesthesia and Analgesia. 2017;124(2):398-405.

26. Sheth T, Chan M, Butler C, Chow B, Tandon V, Nagele P, et al. Prognostic capabilities of coronary computed tomographic angiography before non-cardiac surgery: prospective cohort study. BMJ-British Medical Journal. 2015;350.

27. Weber M, Luchner A, Manfred S, Mueller C, Liebetrau C, Schlitt A, et al. Incremental value of high-sensitive troponin $\mathrm{T}$ in addition to the revised cardiac index for peri-operative risk stratification in non-cardiac surgery. European Heart Journal. 2013;34(11):853-62.

28. Park S-J, Choi J-H, Cho S-J, Chang S-A, Choi J-O, Lee S-C, et al. Comparison of Transthoracic Echocardiography With N-Terminal Pro-Brain Natriuretic Peptide as a Tool for Risk Stratification of Patients Undergoing Major Noncardiac Surgery. Korean Circulation Journal. 2011;41(9):505-11. 
29. van Waes JAR, van Klei WA, Wijeysundera DN, van Wolfswinkel L, Lindsay TF, Beattie WS. Association between Intraoperative Hypotension and Myocardial Injury after Vascular Surgery. Anesthesiology. 2016;124(1):35-44.

30. Bijker JB, Persoon S, Peelen LM, Moons KG, Kalkman CJ, Kappelle LJ, et al. Intraoperative hypotension and perioperative ischemic stroke after general surgery: a nested case-control study. Anesthesiology. 2012;116(3):658-64.

31. Wesselink EM, Kappen TH, Torn HM, Slooter AJC, van Klei WA. Intraoperative hypotension and the risk of postoperative adverse outcomes: a systematic review. Br J Anaesth. 2018;121(4):706-21.

32. Salmasi V, Maheshwari K, Yang D, Mascha EJ, Singh A, Sessler DI, et al. Relationship between Intraoperative Hypotension, Defined by Either Reduction from Baseline or Absolute Thresholds, and Acute Kidney and Myocardial Injury after Noncardiac Surgery: A Retrospective Cohort Analysis. Anesthesiology. 2017;126(1):47-65.

33. Bijker JB, van Klei WA, Kappen TH, van Wolfswinkel L, Moons KG, Kalkman CJ. Incidence of intraoperative hypotension as a function of the chosen definition: literature definitions applied to a retrospective cohort using automated data collection. Anesthesiology. 2007;107(2):213-20.

34. Sessler DI, Bloomstone JA, Aronson S, Berry C, Gan TJ, Kellum JA, et al. Perioperative Quality Initiative consensus statement on intraoperative blood pressure, risk and outcomes for elective surgery. Br J Anaesth. 2019;122(5):563-74.

35. Grobben RB, van Waes JAR, Leiner T, Peelen LM, de Borst GJ, Vogely HC, et al. Unexpected Cardiac Computed Tomography Findings in Patients With Postoperative Myocardial Injury. Anesthesia and Analgesia. 2018;126(5):1462-8.

36. Noordzij PG, van Geffen O, Dijkstra IM, Boerma D, Meinders AJ, Rettig TCD, et al.High-sensitive cardiac troponin T measurements in prediction of non-cardiac complications after major abdominal surgery. British Journal of Anaesthesia. 2015;114(6):909-18 



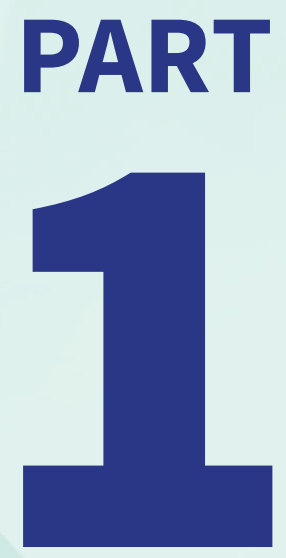

INTRAOPERATIVE HYPOTENSION 



\section{CHAPTER}

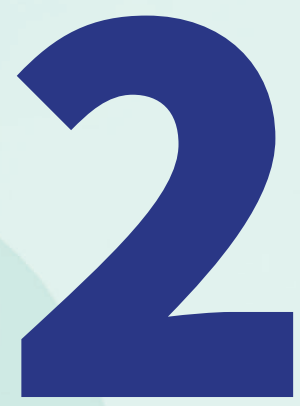

\section{Methods to express intraoperative hypotension exposure in anaesthesia literature}

Lisette M. Vernooij Wilton A. van Klei Karel G. M. Moons Judith A. van Waes Linda M. Peelen 
Editor - Intraoperative hypotension $(\mathrm{IOH})$ has been associated with adverse postoperative outcomes, such as stroke, myocardial injury, acute kidney injury and mortality. (1-5) Despite a recent consensus definition of $\mathrm{IOH}$ for clinical purposes, (6) there is currently no standardized methodology on how to incorporate severity of $\mathrm{IOH}$ in research. Such uniform methodology is important to enable comparison and aggregation of scientific evidence. (7) As a first step, we aimed to provide an overview of the variety of methods used to express $\mathrm{IOH}$ exposure in anaesthesia literature, with no specific intention to provide guidance on what methods should be used in this research.

We performed a systematic search in PubMed including seven anaesthesia journals (Anaesthesia, Anesthesia and Analgesia, Anesthesiology, British Journal of Anaesthesia, Canadian Journal of Anaesthesia, European Journal of Anaesthesiology and Journal of Clinical Computing and Monitoring). Eligibility criteria were original research papers on studies conducted in the noncardiac surgical population using intraoperative blood pressure (BP) measurements for at least 30 consecutive minutes with a measuring interval of at most 5 minutes, published between January 2007 and July 2018. Methods to express IOH exposure were extracted and allocated to one of eight predefined categories; incidence, number of episodes, duration, fall in BP, area under the threshold (AUT), variability, performance of a closed-loop algorithm (8) and number of hypotensive measurements (definitions in Supplemental material). For each method, we extracted whether it was reported in the Method section of the article. Additionally, we extracted whether $\mathrm{IOH}$ was part of the association of interest (primary aim) or rather was studied as confounder of other primary study associations. We hypothesized that more elaborate methods would be used and described in the Method section, when $\mathrm{IOH}$ was part of the primary aim. Elaborate methods entail more information, i.e. more data points are included in this particular summary statistic (e.g. methods related to AUT), compared to methods which are e.g. based on single measurements, i.e. incidence. Descriptive statistics were used to summarize our results. Studies were compared based on whether $\mathrm{IOH}$ was part of the primary aim of the study, or not. If so, we further stratified articles based on whether $\mathrm{IOH}$ served as either the exposure variable (i.e. determinant) or outcome. Risk of bias assessment was not assessed as we only focused on the methodology to express $1 \mathrm{OH}$ exposure and not on certain associations or effect measures. More detailed information on the search strategy, included papers, study selection and characteristics are reported in the Supplemental material.

Screening of 1,282 titles and abstracts resulted in 237 articles reporting at least one method to summarize $\mathrm{IOH}$ exposure. More methods of modelling $\mathrm{IOH}$ exposure were used if $\mathrm{IOH}$ was part of the primary aim (median 3; IQR [2, 4], compared to articles not including IOH in the primary aim, median 1; IQR [1, 3]) (Table). The most frequently used category was Incidence (90\%) followed by Duration (28\%) and Lowest value (27\%). Incidence of hypotension (72\%) and Vasopressor 
administration (49\%) were the most commonly used methods. Methods were more complex in case $\mathrm{IOH}$ was part of the primary study aim, such as methods from the categories AUT, Variability and Performance. If $\mathrm{IOH}$ was the determinant in the primary association of interest, methods related to Duration and AUT were more often used (83\% versus 30\% and $20 \%$ versus $3 \%$ for $10 \mathrm{H}$ as determinant versus outcome, respectively). For $1 \mathrm{OH}$ as the outcome, methods related to Performance and Number of hypotensive measurements were more frequently used (12\% vs. 0\% and $12 \%$ vs. $4 \%$ for outcome vs. determinant, respectively). Higher reporting rates were observed for more complex methods and in case $\mathrm{IOH}$ was part of the primary aim. For example, all AUT and Performance methods were reported in 100\% of the included articles compared to a reporting rate of $63 \%$ and $52 \%$ for Incidence of hypotension and Lowest measured BP, respectively.

Aggregating evidence on the importance of $\mathrm{IOH}$ is difficult as there is wide variety in methodology used to analyse the severity of $\mathrm{IOH}$, and methods are often not properly reported. Methods tend to be more elaborate when $\mathrm{IOH}$ was part of the primary aim compared to studies not including $\mathrm{IOH}$ in the primary aim while all methods reflex the same measure, namely $\mathrm{IOH}$. Therefore, we believe that standardized definitions of $\mathrm{IOH}$ for research purposes are necessary. This should not depend on whether $\mathrm{IOH}$ is in the primary aim or not.

We previously examined the association between different methods to express $1 \mathrm{OH}$ exposure and postoperative myocardial injury and acute kidney injury. (5) This investigation shows that the method of modelling $\mathrm{IOH}$ considerably influences the strength of the studied association. (5) In addition, a systematic review that investigated the association between $\mathrm{IOH}$ and postoperative adverse events reported that a meta-analysis was not possible due to different patient populations, outcome definitions and considerable variation in thresholds to define and methods to express severity of $\mathrm{IOH}$. (4) Altogether, different conclusions could be drawn from studies investigating similar associations with the usage of both different $\mathrm{IOH}$ thresholds and methods of modelling $\mathrm{IOH}$ exposure.

For certain categories, the BP measurement interval has great impact on the incidence(9) and total exposure of $\mathrm{IOH}$. For example, an intraoperative period of 60 minutes could result in different total hypotensive durations using a BP measurement interval of 1 minute versus 5 minutes in the same patient. Besides the interval used, BP measurements could also be affected by measurement errors or artefacts. (10) This might lead to biased results using methods of modelling $\mathrm{IOH}$ that are more dependent on single measurements, such as Incidence and Lowest value. In addition, we found that methods on BP management by treating anaesthetists using interventions such as fluids, vasopressors or inotropic agents were used in only seven of 237 included studies. As $\mathrm{IOH}$ is a potentially modifiable factor in the operating room, it seems appropriate to include such treatment of low BP in research on $1 \mathrm{OH}$. 
Table 1. Methods to reflect intraoperative hypotension in statistical models with their reporting rates

\section{Overall}

\begin{tabular}{lc}
\hline $\mathrm{n}$ & 237 \\
Total number of categories (median [IQR]) & $1[1,2]$ \\
Total number of methods (median [IQR]) & $2[1,3]$ \\
\hline
\end{tabular}

\section{Method used Reported?}

\begin{tabular}{|c|c|c|}
\hline Presence (\%) & $214(90.3)$ & $127(59.3)$ \\
\hline Incidence of hypotension & $155(72.4)$ & $98(63.2)$ \\
\hline Vasopressor administration (y/n) & $104(48.6)$ & $57(54.8)$ \\
\hline Incidence double low & $5(2.3)$ & $3(60.0)$ \\
\hline Other presence methods & $4(1.9)$ & $1(25.0)$ \\
\hline Number of episodes (\%) & $37(15.1)$ & $26(70.3)$ \\
\hline Number of episodes & $20(54.1)$ & $14(70.0)$ \\
\hline Number of vasopressor administrations & $20(54.1)$ & $11(55.0)$ \\
\hline Other number of episodes methods & $6(16.2)$ & $6(100.0)$ \\
\hline Duration (\%) & $66(27.8)$ & $48(72.7)$ \\
\hline Total hypotensive duration & $40(60.6)$ & $33(82.5)$ \\
\hline Percentage time outside threshold & $8(12.1)$ & $6(75.0)$ \\
\hline Percentage time within threshold & $6(9.1)$ & $5(83.3)$ \\
\hline Maximum episode duration & $7(10.6)$ & $6(85.7)$ \\
\hline Average episode duration & $5(7.6)$ & $4(80.0)$ \\
\hline Time to administration vasopressor & $5(7.6)$ & $4(80.0)$ \\
\hline Other duration methods & $25(37.9)$ & $19(76.0)$ \\
\hline$A \cup C(\%)$ & $9(3.8)$ & $9(100.0)$ \\
\hline AUC & $9(100.0)$ & $9(100.0)$ \\
\hline Other AUC methods & $5(55.6)$ & $5(100.0)$ \\
\hline Lowest value (\%) & $63(26.6)$ & $33(52.4)$ \\
\hline Lowest measured BP & $46(73.0)$ & $24(52.1)$ \\
\hline Relative fall below baseline & $20(31.7)$ & $13(65.0)$ \\
\hline Absolute fall below baseline & $7(11.1)$ & $2(28.6)$ \\
\hline Absolute fall below threshold & $5(7.9)$ & $4(80.0)$ \\
\hline Other lowest value methods & $9(14.3)$ & $8(88.9)$ \\
\hline
\end{tabular}


Primary study aim

$\mathrm{IOH}$ not in primary aim $\mathrm{IOH}$ in primary aim
93

$2[2,3]$

$3[2,4]$

\section{Role of $\mathrm{IOH}$ variable}

Determinant

Outcome

24

69

$3[2,4]$

$2[1.3,3]$

$1[1,2]$
$3[2,5]$
$3[2,4]$

\begin{tabular}{cccccccc}
$\begin{array}{c}\text { Method } \\
\text { used }\end{array}$ & Reported? & used & Reported? & used & Reported? & used & Reported? \\
\hline $133(92.4)$ & $65(48.9)$ & $81(87.1)$ & $62(76.5)$ & $21(87.5)$ & $16(76.2)$ & $60(87.0)$ & $46(76.7)$ \\
$85(63.9)$ & $44(51.8)$ & $70(86.4)$ & $54(77.1)$ & $17(81.0)$ & $13(81.3)$ & $53(88.3)$ & $41(77.4)$ \\
$71(53.3)$ & $31(43.7)$ & $33(40.7)$ & $26(78.8)$ & $5(23.8)$ & $4(80)$ & $28(46.7)$ & $22(78.6)$ \\
$2(1.5)$ & $1(50.0)$ & $3(3.7)$ & $2(66.7)$ & $2(9.5)$ & $1(50.0)$ & $1(1.7)$ & $1(100.0)$ \\
$2(1.5)$ & $0(0.0)$ & $2(2.1)$ & $1(50.0)$ & $2(9.5)$ & $1(50.0)$ & $0(0.0)$ & $N A$ \\
$12(8.3)$ & $6(50.0)$ & $25(26.9)$ & $20(80.0)$ & $6(25.0)$ & $6(100.0)$ & $19(27.5)$ & $14(73.7)$ \\
$5(41.7)$ & $3(60.0)$ & $15(60.0)$ & $11(73.3)$ & $3(50.0)$ & $3(100.0)$ & $12(63.2)$ & $8(66.7)$ \\
$8(66.7)$ & $2(25.0)$ & $12(48.0)$ & $9(75.0)$ & $1(16.7)$ & $1(100.0)$ & $11(57.9)$ & $8(72.7)$ \\
$1(8.3)$ & $1(100.0)$ & $5(20.0)$ & $5(100.0)$ & $4(66.7)$ & $4(100.0)$ & $1(5.3)$ & $1(100.0)$ \\
$25(17.4)$ & $15(60.0)$ & $41(44.1)$ & $33(80.5)$ & $20(83.3)$ & $17(85.0)$ & $21(30.4)$ & $16(76.2)$ \\
$12(48.0)$ & $9(75.0)$ & $28(68.3)$ & $24(85.7)$ & $17(85.0)$ & $15(88.2)$ & $11(52.4)$ & $9(81.8)$ \\
$5(20.0)$ & $3(60.0)$ & $3(7.3)$ & $3(100.0)$ & $1(5.0)$ & $1(100.0)$ & $2(9.5)$ & $2(100.0)$ \\
$3(12.0)$ & $2(66.7)$ & $3(7.3)$ & $3(100.0)$ & $0(0.0)$ & $N A$ & $3(14.3)$ & $3(100.0)$ \\
$0(0.0)$ & $N A$ & $7(17.1)$ & $6(85.7)$ & $3(15.0)$ & $2(66.7)$ & $4(19.0)$ & $4(100.0)$ \\
$1(4.0)$ & $0(0.0)$ & $4(9.8)$ & $4(100.0)$ & $2(10.0)$ & $2(100.0)$ & $2(9.5)$ & $2(100.0)$ \\
$1(4.0)$ & $1(100.0)$ & $4(9.8)$ & $3(75.0)$ & $1(5.0)$ & $1(100.0)$ & $3(4.3)$ & $2(66.7)$ \\
$8(32.0)$ & $5(62.5)$ & $17(41.5)$ & $14(82.4)$ & $6(30.0)$ & $4(66.7)$ & $11(52.4)$ & $9(81.2)$ \\
$2(1.4)$ & $2(100.0)$ & $7(7.5)$ & $7(100.0)$ & $5(20.8)$ & $5(100.0)$ & $2(2.9)$ & $2(100.0)$ \\
$2(100.0)$ & $2(100.0)$ & $7(100.0)$ & $7(100.0)$ & $5(100.0)$ & $5(100.0)$ & $2(100.0)$ & $2(100.0)$ \\
$0(0.0)$ & $N A$ & $5(71.4)$ & $5(100.0)$ & $4(80.0)$ & $4(100.0)$ & $1(50.0)$ & $1(100.0)$ \\
$24(16.7)$ & $10(41.7)$ & $39(41.9)$ & $23(59.0)$ & $10(41.7)$ & $9(90.0)$ & $29(42.0)$ & $14(14.3)$ \\
$19(79.1)$ & $8(42.1)$ & $27(69.2)$ & $16(59.3)$ & $8(80.0)$ & $7(87.5)$ & $19(65.5)$ & $9(47.4)$ \\
$7(19.2)$ & $3(42.9)$ & $13(33.3)$ & $10(76.9)$ & $2(20.0)$ & $2(100.0)$ & $11(37.9)$ & $8(72.7)$ \\
$3(12.5)$ & $1(33.3)$ & $4(10.3)$ & $1(25.0$ & $0(0.0)$ & $N A$ & $4(13.8)$ & $1(25.0)$ \\
$3(12.5)$ & $2(66.7)$ & $2(5.1)$ & $2(100.0)$ & $1(10.0)$ & $1(100.0)$ & $1(3.4)$ & $1(100.0)$ \\
$3(12.5)$ & $3(100.0)$ & $6(15.4)$ & $5(83.3)$ & $3(30.0)$ & $3(100.0)$ & $3(10.3)$ & $2(66.7)$ \\
\hline 10.0
\end{tabular}


Table 1. Methods to reflect intraoperative hypotension in statistical models with their reporting rates

\section{Overall}

\begin{tabular}{lc}
$\mathrm{n}$ & 237 \\
Total number of categories (median [IQR]) & $1[1,2]$ \\
Total number of methods (median [IQR]) & $2[1,3]$ \\
\hline
\end{tabular}

Method used Reported?

\begin{tabular}{lcc}
\hline Variability (\%) & $10(4.2)$ & $7(70.0)$ \\
SD of BP & $6(60.0)$ & $3(50.0)$ \\
Other variability methods & $8(80.0)$ & $8(100.0)$ \\
Performance (\%)* & $11(4.6)$ & $11(100.0)$ \\
Performance error & $11(100.0)$ & $11(100.0)$ \\
Median performance error & $10(90.9)$ & $10(100.0)$ \\
Median absolute performance error & $9(91.8)$ & $9(100.0)$ \\
Wobble & $8(72.7)$ & $8(100.0)$ \\
Divergence & $6(54.5)$ & $6(100.0)$ \\
Other performance methods & $2(18.2)$ & $2(100.0)$ \\
Number of measurements (\%) & $10(4.2)$ & $4(40.0)$ \\
Absolute number of measurements outside the threshold & $6(60.0)$ & $4(66.7)$ \\
Other number of measurements methods & $7(70.0)$ & $0(0.0)$ \\
\hline
\end{tabular}

Data is presented as $n(\%)$ or as median [interquartile range (IQR)]. * For the Performance methods, see Sng et al(8) for a more detailed description. IOH: intraoperative hypotension; IQR: interquartile range; AUT: area under the threshold; BP: blood pressure; SD: standard deviation; NA: not applicable. 
Primary study aim

$\mathrm{IOH}$ not in primary aim $\mathrm{IOH}$ in primary aim

144

$1[1,2]$

$1[1,2]$

Method

used

93

$2[2,3]$

$3[2,4]$
Role of $10 \mathrm{H}$ variable

Determinant

Outcome

24

$3[2,4]$

69

$2[1.3,3]$

$3[2,5]$

$3[2,4]$

\begin{tabular}{cccccccc}
$1(0.7)$ & $0(0.0)$ & $9(9.7)$ & $7(77.8)$ & $3(12.5)$ & $3(100.0)$ & $6(8.7)$ & $4(66.7)$ \\
$1(100.0)$ & $0(0.0)$ & $5(55.6)$ & $3(60.0)$ & $2(66.7)$ & $2(100.0)$ & $3(50.0)$ & $1(33.3)$ \\
$0(0.0)$ & $N A$ & $8(88.9)$ & $8(100.0)$ & $4(133.3)$ & $4(100.0)$ & $4(66.7)$ & $4(100.0)$ \\
$3(2.1)$ & $3(100.0)$ & $8(8.6)$ & $8(100.0)$ & $0(0.0)$ & NA & $8(11.6)$ & $8(100.0)$ \\
$3(100.0)$ & $3(100.0)$ & $8(100.0)$ & $8(100.0)$ & $0(0.0)$ & NA & $8(100.0)$ & $8(100.0)$ \\
$2(66.7)$ & $2(100.0)$ & $8(100.0)$ & $8(100.0)$ & $0(0.0)$ & $N A$ & $8(100.0)$ & $8(100.0)$ \\
$1(33.3)$ & $1(100.0)$ & $8(100.0)$ & $8(100.0)$ & $0(0.0)$ & $N A$ & $8(100.0)$ & $8(100.0)$ \\
$1(33.3)$ & $1(100.0)$ & $7(87.5)$ & $7(100.0)$ & $0(0.0)$ & $N A$ & $7(87.5)$ & $7(100.0)$ \\
$1(33.3)$ & $1(100.0)$ & $5(62.5)$ & $5(100.0)$ & $0(0.0)$ & $N A$ & $5(62.5)$ & $5(100.0)$ \\
$0(0.0)$ & $N A$ & $2(25.0)$ & $2(100.0)$ & $0(0.0)$ & $N A$ & $2(25.0)$ & $2(100.0)$ \\
$1(0.7)$ & $0(0.0)$ & $9(9.7)$ & $4(44.4)$ & $1(4.2)$ & $0(0.0)$ & $8(11.6)$ & $4(50.0)$ \\
$0(0.0)$ & $N A$ & $6(66.7)$ & $4(66.7)$ & $0(0.0)$ & NA & $6(75.0)$ & $4(66.7)$ \\
$1(100.0)$ & $0(0.0)$ & $6(66.7)$ & $1(16.7)$ & $1(100.0)$ & $0(0.0)$ & $5(62.5)$ & $1(20.0)$ \\
\hline
\end{tabular}


One might argue that for different surgical populations and settings (i.e. general or spinal anaesthesia, or use of a closed-loop-system (8)), different methods to express $10 H$ should be used. For example, studying the association between BP and outcomes in a general surgical population receiving general anaesthesia may require methods such as duration and/or AUT, whereas investigating the effect of spinal anaesthesia on BP in obstetric patients may require incidences or lowest BP measured. Consequently, there should be special emphasis on different domains for consensus on standardized methodology to summarize $\mathrm{IOH}$ exposure for research purposes.

In summary, there is considerable variation in methods used to express severity of $\mathrm{IOH}$, which might hamper interpretation and comparison of study findings. However, we were not able to provide any guidance on what methods should be used based on the current literature. Standardized definitions of $\mathrm{IOH}$ should be based on both threshold and method to express $\mathrm{IOH}$ severity and should include clear reporting guidance for research purposes to improve comparability and reproducibility of different studies. 


\section{REFERENCES}

1. Walsh M, Devereaux PJ, Garg AX, Kurz A, Turan A, Rodseth RN, et al. Relationship between intraoperative mean arterial pressure and clinical outcomes after noncardiac surgery: toward an empirical definition of hypotension. Anesthesiology [Internet]. 2013 Sep [cited 2015 Feb 23];119(3):507-15. Available from: http://www.ncbi.nlm.nih.gov/pubmed/23835589

2. Monk TG, Bronsert MR, Henderson WG, Mangione MP, Sum-Ping STJT, Bentt DR, et al. Association between Intraoperative Hypotension and Hypertension and 30-day Postoperative Mortality in Noncardiac Surgery. Anesthesiology [Internet]. 2015 Jun 17 [cited 2015 Jun 23]; Available from: http://www.ncbi.nlm.nih.gov/pubmed/26083768

3. Salmasi V, Maheshwari K, Yang D, Mascha EJ, Singh A, Sessler DI, et al. Relationship between Intraoperative Hypotension, Defined by Either Reduction from Baseline or Absolute Thresholds, and Acute Kidney and Myocardial Injury after Noncardiac Surgery: A Retrospective Cohort Analysis. Anesthesiology [Internet]. 2017 Jan [cited 2017 Jul 31];126(1):47-65. Available from: http://insights.ovid.com/crossref?an=00000542-201701000-00017

4. Wesselink EM, Kappen TH, Torn HM, Slooter AJC, van Klei WA. Intraoperative hypotension and the risk of postoperative adverse outcomes: a systematic review. $\mathrm{Br} J$ Anaesth [Internet]. 2018 Oct [cited 2019 Jan 17];121(4):706-21. Available from: http://www.ncbi.nlm.nih.gov/ pubmed/30236233

5. Vernooij LM, van Klei WA, Machina M, Pasma W, Beattie WS, Peelen LM. Different methods of modelling intraoperative hypotension and their association with postoperative complications in patients undergoing non-cardiac surgery. Br J Anaesth [Internet]. 2018 May [cited 2019 Jan 17];120(5):1080-9. Available from: https://linkinghub.elsevier.com/retrieve/ pii/S0007091218301004

6. Sessler DI, Bloomstone JA, Aronson S, Berry C, Gan TJ, Kellum JA, et al. Perioperative Quality Initiative consensus statement on intraoperative blood pressure, risk and outcomes for elective surgery. Br J Anaesth [Internet]. 2019 Feb 27 [cited 2019 Apr 9];0(0). Available from: http://www.ncbi.nlm.nih.gov/pubmed/30916004

7. Deeks JJ, Higgins JP, Altman DG. Analysing Data and Undertaking Meta-Analyses. In: Cochrane Handbook for Systematic Reviews of Interventions [Internet]. Chichester, UK: John Wiley \& Sons, Ltd; [cited 2019 Feb 5]. p. 243-96. Available from: http://doi.wiley. com/10.1002/9780470712184.ch9

8. Sng BL, Wang H, Assam PN, Sia AT. Assessment of an updated double-vasopressor automated system using Nexfin ${ }^{\mathrm{M}}$ for the maintenance of haemodynamic stability to improve perioperative outcome during spinal anaesthesia for caesarean section. Anaesthesia [Internet]. 2015 Jun [cited 2019 Feb 7];70(6):691-8. Available from: http://doi.wiley.com/10.1111/ anae. 13008

9. Kruger GH, Shanks A, Kheterpal S, Tremper T, Chiang C-J, Freundlich RE, et al. Influence of non-invasive blood pressure measurement intervals on the occurrence of intra-operative hypotension. J Clin Monit Comput [Internet]. 2018 Aug 30 [cited 2019 Feb 7];32(4):699-705. Available from: http://link.springer.com/10.1007/s10877-017-0065-4 
10. Kool NP, van Waes JAR, Bijker JB, Peelen LM, van Wolfswinkel L, de Graaff $J C$, et al. Artifacts in research data obtained from an anesthesia information and management system. Can J Anaesth [Internet]. 2012 Sep [cited 2015 Jul 10];59(9):833-41. Available from: http://www.pubmedcentral.nih.gov/articlerender. fcgi?artid=3425740\&tool=pmcentrez\&rendertype=abstract 


\section{SUPPLEMENTAL MATERIAL}

Supplementary section 1. Category definitions of $\mathrm{IOH}$ methods

Incidence of $1 \mathrm{OH}$, which is defined as a dichotomous variable for being hypotensive during surgery at least once.

- Number of episodes, defined as the number of separate episodes in which the intraoperative blood pressure was below the predefined threshold

- Time related methods include methods using time as part of the total amount of $1 \mathrm{OH}$ exposure, for example total hypotensive duration, time between hypotensive episodes and maximal episode duration. The $\mathrm{IOH}$ exposure is most frequently presented in minutes.

- Fall in blood pressure is defined as a decline in blood pressure from a predefined blood pressure level, such as baseline blood pressure or a blood pressure threshold to define $\mathrm{IOH}$. $\mathrm{IOH}$ exposure for each of these methods is presented as $\mathrm{mm} \mathrm{Hg}$.

- $\quad$ Area under the curve (AUC) or area under the threshold is defined as the combination of time and decline in blood pressure below a certain threshold. Data for these methods are most frequently presented as $\mathrm{min}^{*} \mathrm{~mm} \mathrm{Hg}$.

- Variability of blood pressure includes methods related to the amount of dispersion of blood pressure measurements during surgery, such as the standard deviation and variance.

- Performance of a closed-loop algorithm for blood pressure maintenance, in which such system allows for greater control of hemodynamic status during anaesthesia. More detailed information concerning these methods is reported in Sng et $a^{8}$.

- Number of hypotensive measurements is defined as the amount of hypotensive measurements during surgery. These methods were most commonly presented as either the absolute number of measurements or proportion of hypotensive measurements relative to the total number of blood pressure measurements during surgery. 
Supplementary section 2. PubMed search strategy

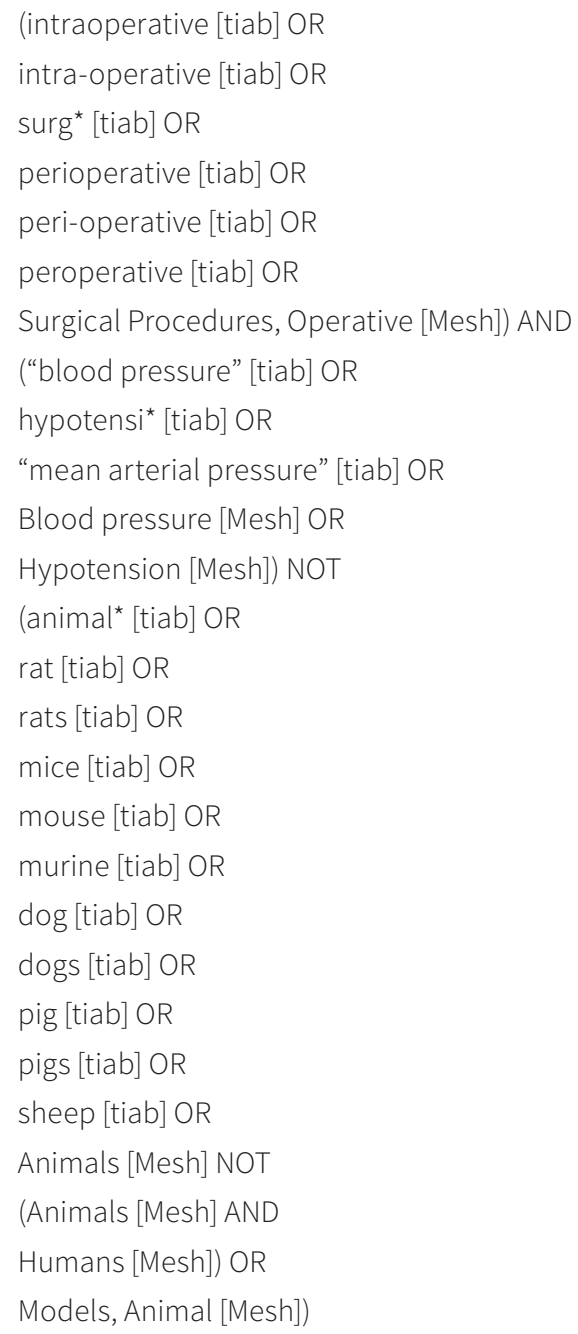


Supplemental Figure 1. Flow diagram of selected articles

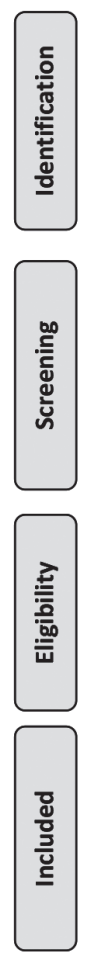

Selected articles from seven anaesthesiology journals from 1-1-2007 to 1-7-2018 $(n=1282)$

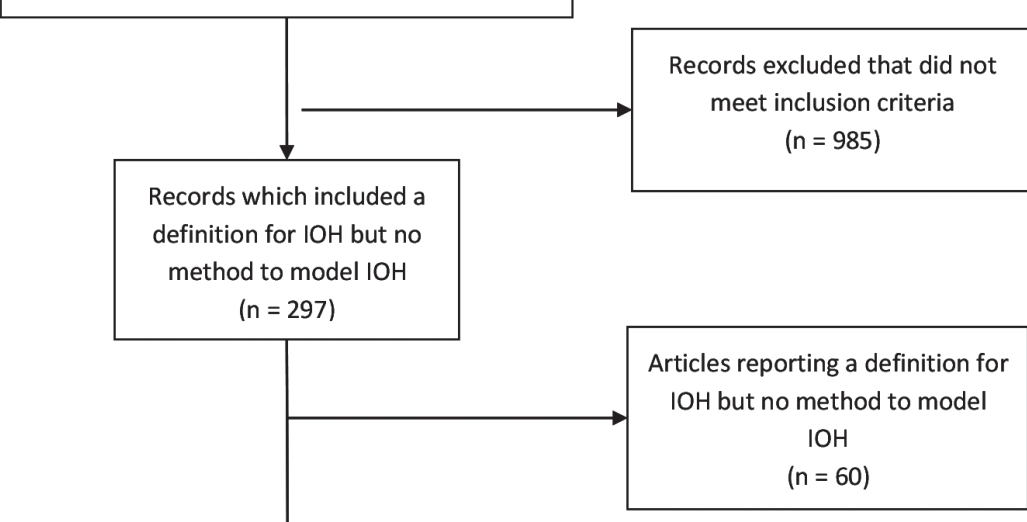

Articles that included both a definition for and a method to model $\mathrm{IOH}$ $(n=237)$ 


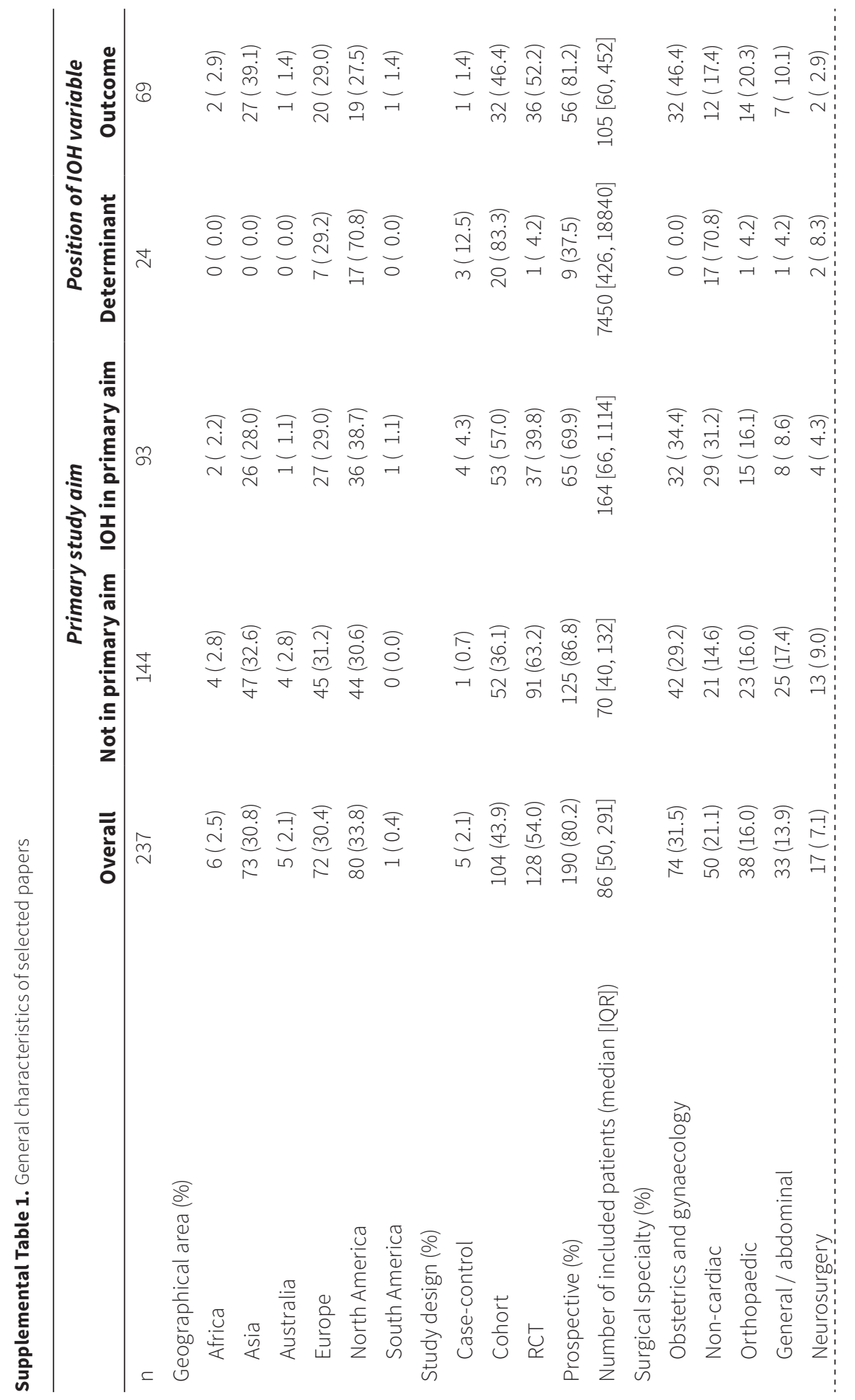




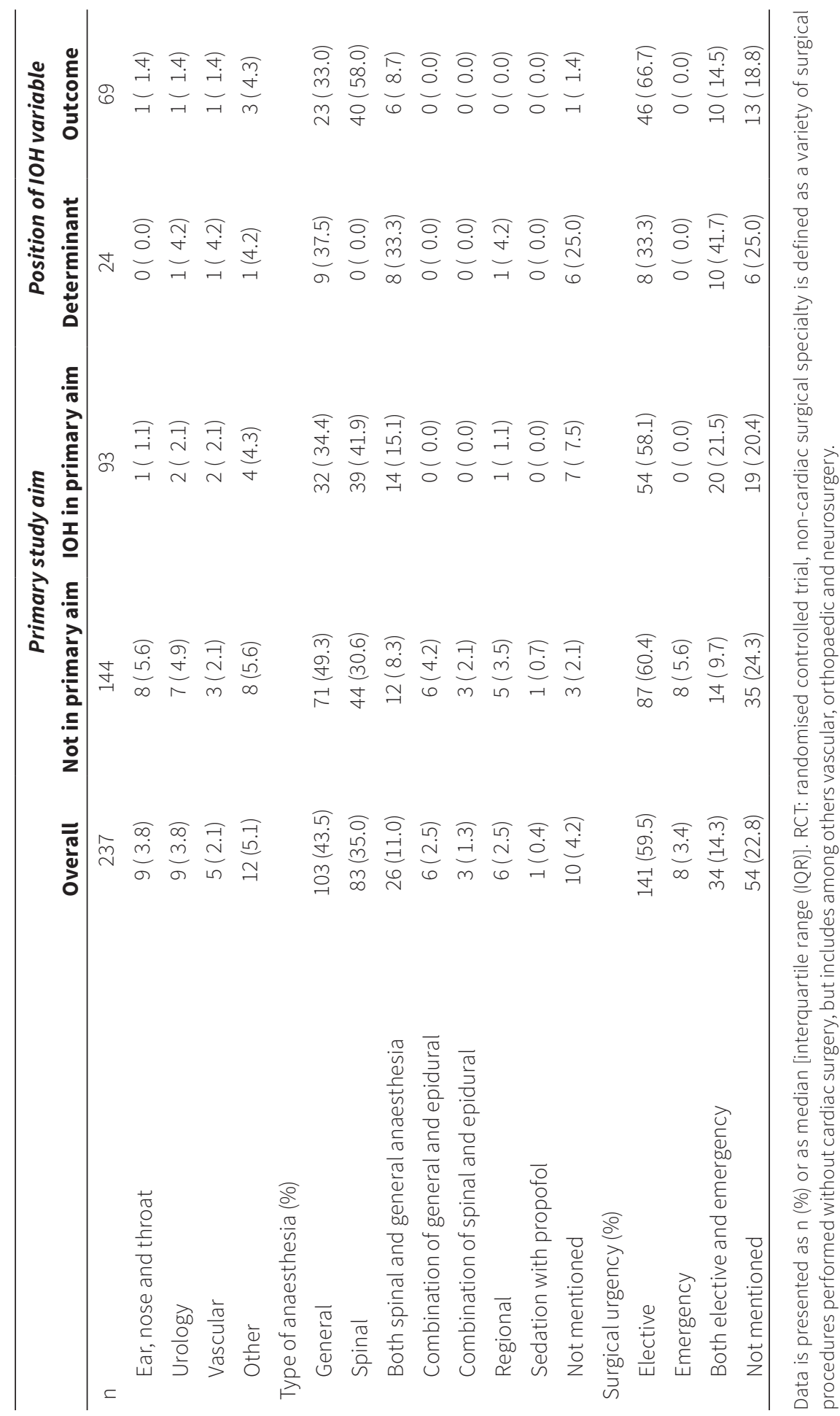


Supplemental section 3. List of included studies

1. Benes J, Simanova A, Tovarnicka T, et al. Continuous non-invasive monitoring improves blood pressure stability in upright position: randomized controlled trial. J Clin Monit Comput. 2015;29(1):11-17. doi:10.1007/s10877-014-9586-2

2. Kumar A, Chen Y, Lin H-M, Deiner S. Elevated preoperative blood pressure predicts the intraoperative loss of SSEP neuromonitoring signals during spinal surgery. J Clin Monit Comput. 2014;28(2):187-192. doi:10.1007/s10877-013-9515-9

3. Spruit RJ, Schwarte LA, Hakenberg OW, Scheeren TWL. Association of intraoperative tissue oxygenation with suspected risk factors for tissue hypoxia. J Clin Monit Comput. 2013;27(5):541-550. doi:10.1007/s10877-013-9460-7

4. Rossi M, Cividjian A, Fevre MC, et al. A beat-by-beat, on-line, cardiovascular index, CARDEAN, to assess circulatory responses to surgery: a randomized clinical trial during spine surgery. J Clin Monit Comput. 2012;26(6):441-449. doi:10.1007/s10877-012-9372-y

5. Chen G, Chung E, Meng L, et al. Impact of non invasive and beat-to-beat arterial pressure monitoring on intraoperative hemodynamic management. J Clin Monit Comput. 2012;26(2):133-140. doi:10.1007/s10877-012-9344-2

6. Hegde HV, Puri GD, Kumar B, Behera A. Bi-spectral index guided closed-loop anaesthesia delivery system (CLADS) in pheochromocytoma. J Clin Monit Comput. 2009;23(4):189-196. doi:10.1007/s10877-009-9181-0

7. Sng BL, Wang H, Assam PN, Sia AT. Assessment of an updated double-vasopressor automated system using Nexfin for the maintenance of haemodynamic stability to improve peri-operative outcome during spinal anaesthesia for caesarean section. Anaesthesia. 2015;70(6):691-698. doi:10.1111/anae.13008

8. Sng BL, Tan HS, Sia ATH. Closed-loop double-vasopressor automated system vs manual bolus vasopressor to treat hypotension during spinal anaesthesia for caesarean section: a randomised controlled trial. Anaesthesia. 2014;69(1):37-45. doi:10.1111/anae.12460

9. Daher M, Saito RB, Barra GB, Govêia CS, Magalhães E, Neves FAR. The effect of beta-2 adrenergic receptor haplotype variations on the haemodynamic response following spinal anaesthesia for caesarean delivery. Anaesthesia. 2012;67(11):1251-1259. doi:10.1111/j.13652044.2012.07296.x

10. Sia ATH, Tan HS, Sng BL. Closed-loop double-vasopressor automated system to treat hypotension during spinal anaesthesia for caesarean section: a preliminary study. Anaesthesia. 2012;67(12):1348-1355. doi:10.1111/anae.12000

11. Wood RJ, White SM. Anaesthesia for 1131 patients undergoing proximal femoral fracture repair: a retrospective, observational study of effects on blood pressure, fluid administration and perioperative anaemia. Anaesthesia. 2011;66(11):1017-1022. doi:10.1111/j.13652044.2011.06854.x

12. Ngan Kee WD, Khaw KS, Lau TK, Ng FF, Chui K, Ng KL. Randomised double-blinded comparison of phenylephrine vs ephedrine for maintaining blood pressure during spinal anaesthesia for non-elective Caesarean section*. Anaesthesia. 2008;63(12):1319-1326. doi:10.1111/j.1365-2044.2008.05635.x

13. Kundra P, Khanna S, Habeebullah S, Ravishankar M. Manual displacement of the uterus during Caesarean section. Anaesthesia. 2007;62(5):460-465. doi:10.1111/j.1365-2044.2007.05025.x 
14. Ngan Kee WD, Tam YH, Khaw KS, Ng FF, Critchley LA, Karmakar MK. Closed-loop feedback computer-controlled infusion of phenylephrine for maintaining blood pressure during spinal anaesthesia for caesarean section: a preliminary descriptive study. Anaesthesia. 2007;62(12):1251-1256. doi:10.1111/j.1365-2044.2007.05257.x

15. Puri GD, Mathew PJ, Biswas I, et al. A Multicenter Evaluation of a Closed-Loop Anesthesia Delivery System: A Randomized Controlled Trial. Anesth Analg. April 2015. doi:10.1213/ ANE.0000000000000769

16. Spielberg DR, Barrett JS, Hammer GB, et al. Predictors of arterial blood pressure control during deliberate hypotension with sodium nitroprusside in children. Anesth Analg. 2014;119(4):867-874. doi:10.1213/ANE.0000000000000376

17. Siddik-Sayyid SM, Taha SK, Kanazi GE, Aouad MT. A randomized controlled trial of variable rate phenylephrine infusion with rescue phenylephrine boluses versus rescue boluses alone on physician interventions during spinal anesthesia for elective cesarean delivery. Anesth Analg. 2014;118(3):611-618. doi:10.1213/01.ane.0000437731.60260.ce

18. Nair BG, Horibe M, Newman S-F, Wu W-Y, Peterson GN, Schwid HA. Anesthesia information management system-based near real-time decision support to manage intraoperative hypotension and hypertension. Anesth Analg. 2014;118(1):206-214. doi:10.1213/ ANE.0000000000000027

19. Pin-on P, Schroeder D, Munis J. The hemodynamic management of 5177 neurosurgical and orthopedic patients who underwent surgery in the sitting or "beach chair" position without incidence of adverse neurologic events. Anesth Analg. 2013;116(6):1317-1324. doi:10.1213/ ANE.0b013e31828446bb

20. Stricker PA, Lin EE, Fiadjoe JE, et al. Evaluation of central venous pressure monitoring in children undergoing craniofacial reconstruction surgery. Anesth Analg. 2013;116(2):411-419. doi:10.1213/ANE.0b013e31827008e6

21. Stricker PA, Lin EE, Fiadjoe JE, Sussman EM, Jobes DR. Absence of tachycardia during hypotension in children undergoing craniofacial reconstruction surgery. Anesth Analg. 2012;115(1):139-146. doi:10.1213/ANE.0b013e318253708c

22. van der Linden P, Gazdzik TS, Jahoda D, et al. A double-blind, randomized, multicenter study of MP4OX for treatment of perioperative hypotension in patients undergoing primary hip arthroplasty under spinal anesthesia. Anesth Analg. 2011;112(4):759-773. doi:10.1213/ ANE.0b013e31820c7b5f

23. Stewart A, Fernando R, McDonald S, Hignett R, Jones T, Columb M. The dose-dependent effects of phenylephrine for elective cesarean delivery under spinal anesthesia. Anesth Analg. 2010;111(5):1230-1237. doi:10.1213/ANE.0b013e3181f2eae1

24. Allen TK, George RB, White WD, Muir HA, Habib AS. A double-blind, placebo-controlled trial of four fixed rate infusion regimens of phenylephrine for hemodynamic support during spinal anesthesia for cesarean delivery. Anesth Analg. 2010;111(5):1221-1229. doi:10.1213/ ANE.0b013e3181e1db21

25. Bebawy JF, Zeeni C, Sharma S, et al. Adenosine-induced flow arrest to facilitate intracranial aneurysm clip ligation does not worsen neurologic outcome. Anesth Analg. 2013;117(5):12051210. doi:10.1213/ANE.0b013e3182a6d31b 
26. Teoh WHL, Sia ATH. Colloid Preload Versus Coload for Spinal Anesthesia for Cesarean Delivery: The Effects on Maternal Cardiac Output. Anesth Analg. 2009;108(5):1592-1598. doi:10.1213/ane.0b013e31819e016d

27. Haentjens LL, Ghoundiwal D, Touhiri K, et al. Does infusion of colloid influence the occurrence of postoperative nausea and vomiting after elective surgery in women? Anesth Analg. 2009;108(6):1788-1793. doi:10.1213/ane.0b013e3181a1968c

28. Bekker A, Sturaitis M, Bloom M, et al. The effect of dexmedetomidine on perioperative hemodynamics in patients undergoing craniotomy. Anesth Analg. 2008;107(4):1340-1347. doi:10.1213/ane.0b013e3181804298

29. Ngan Kee WD, Lee A, Khaw KS, Ng FF, Karmakar MK, Gin T. A randomized double-blinded comparison of phenylephrine and ephedrine infusion combinations to maintain blood pressure during spinal anesthesia for cesarean delivery: the effects on fetal acid-base status and hemodynamic control. Anesth Analg. 2008;107(4):1295-1302. doi:10.1213/ ane.0b013e31818065bc

30. Monk TG, Bronsert MR, Henderson WG, et al. Association between Intraoperative Hypotension and Hypertension and 30-day Postoperative Mortality in Noncardiac Surgery. Anesthesiology. June 2015. doi:10.1097/ALN.0000000000000756

31. Mascha EJ, Yang D, Weiss S, Sessler DI. Intraoperative Mean Arterial Pressure Variability and 30-day Mortality in Patients Having Noncardiac Surgery. Anesthesiology. 2015;123(1):79-91. doi:10.1097/ALN.0000000000000686

32. Ngan Kee WD, Lee SWY, Ng FF, Tan PE, Khaw KS. Randomized Double-blinded Comparison of Norepinephrine and Phenylephrine for Maintenance of Blood Pressure during Spinal Anesthesia for Cesarean Delivery. Anesthesiology. 2015;122(4):736-745. doi:10.1097/ ALN.0000000000000601

33. Kertai MD, White WD, Gan TJ. Cumulative duration of "triple low" state of low blood pressure, low bispectral index, and low minimum alveolar concentration of volatile anesthesia is not associated with increased mortality. Anesthesiology. 2014;121(1):18-28. doi:10.1097/ ALN.0000000000000281

34. Walsh M, Devereaux PJ, Garg AX, et al. Relationship between intraoperative mean arterial pressure and clinical outcomes after noncardiac surgery: toward an empirical definition of hypotension. Anesthesiology. 2013;119(3):507-515. doi:10.1097/ALN.0b013e3182a10e26

35. Bishop SM, Yarham SI, Navapurkar VU, Menon DK, Ercole A. Multifractal analysis of hemodynamic behavior: intraoperative instability and its pharmacological manipulation. Anesthesiology. 2012;117(4):810-821. doi:10.1097/ALN.0b013e31826a4aa2

36. Bijker JB, Persoon S, Peelen LM, et al. Intraoperative hypotension and perioperative ischemic stroke after general surgery: a nested case-control study. Anesthesiology. 2012;116(3):658664. doi:10.1097/ALN.0b013e3182472320

37. Sessler DI, Sigl JC, Kelley SD, et al. Hospital stay and mortality are increased in patients having a "triple low" of low blood pressure, low bispectral index, and low minimum alveolar concentration of volatile anesthesia. Anesthesiology. 2012;116(6):1195-1203. doi:10.1097/ ALN.0b013e31825683dc

38. Wax DB, Lin H-M, Reich DL. Intraoperative non-record-keeping usage of anesthesia information management system workstations and associated hemodynamic variability and aberrancies. Anesthesiology. 2012;117(6):1184-1189. doi:10.1097/ALN.0b013e3182751715 
39. Olofsson Cl, Górecki AZ, Dirksen R, et al. Evaluation of MP4OX for prevention of perioperative hypotension in patients undergoing primary hip arthroplasty with spinal anesthesia: a randomized, double-blind, multicenter study. Anesthesiology. 2011;114(5):1048-1063. doi:10.1097/ALN.0b013e318215e198

40. Kiefer N, Theis J, Putensen-Himmer G, Hoeft A, Zenker S. Peristaltic pneumatic compression of the legs reduces fluid demand and improves hemodynamic stability during surgery: a randomized, prospective study. Anesthesiology. 2011;114(3):536-544. doi:10.1097/ ALN.0b013e31820c3973

41. Yocum GT, Gaudet JG, Teverbaugh LA, et al. Neurocognitive performance in hypertensive patients after spine surgery. Anesthesiology. 2009;110(2):254-261. doi:10.1097/ ALN.0b013e3181942c7a

42. Bijker JB, van Klei WA, Vergouwe $Y$, et al. Intraoperative hypotension and 1-year mortality after noncardiac surgery. Anesthesiology. 2009;111(6):1217-1226. doi:10.1097/ ALN.0b013e3181c14930

43. Langesaeter E, Rosseland LA, Stubhaug A. Continuous invasive blood pressure and cardiac output monitoring during cesarean delivery: a randomized, double-blind comparison of low-dose versus high-dose spinal anesthesia with intravenous phenylephrine or placebo infusion. Anesthesiology. 2008;109(5):856-863. doi:10.1097/ALN.0b013e31818a401f

44. Bijker JB, van Klei WA, Kappen TH, van Wolfswinkel L, Moons KGM, Kalkman CJ. Incidence of Intraoperative Hypotension as a Function of the Chosen Definition. Anesthesiology. 2007;107(2):213-220. doi:10.1097/01.anes.0000270724.40897.8e

45. Hirsch J, DePalma G, Tsai TT, Sands LP, Leung JM. Impact of intraoperative hypotension and blood pressure fluctuations on early postoperative delirium after non-cardiac surgery $\dagger$ †. Br J Anaesth. 2015;115(3):418-426. doi:10.1093/bja/aeu458

46. Mercier FJ. 6\% Hydroxyethyl starch (130/0.4) vs Ringer's lactate preloading before spinal anaesthesia for Caesarean delivery. Br J Anaesth. 2015;115(2):328-329. doi:10.1093/bja/aev251

47. Murphy GS, Szokol JW, Avram MJ, et al. Effect of ventilation on cerebral oxygenation in patients undergoing surgery in the beach chair position: a randomized controlled trial. Br J Anaesth. 2014;113(4):618-627. doi:10.1093/bja/aeu109

48. Gruenewald M, Willms S, Broch O, Kott M, Steinfath M, Bein B. Sufentanil administration guided by surgical pleth index vs standard practice during sevoflurane anaesthesia: a randomized controlled pilot study. Br J Anaesth. 2014;112(5):898-905. doi:10.1093/bja/aet485

49. Ngan Kee WD, Khaw KS, Ng FF, Tam YH. Randomized comparison of closed-loop feedback computer-controlled with manual-controlled infusion of phenylephrine for maintaining arterial pressure during spinal anaesthesia for caesarean delivery. Br J Anaesth. 2013;110(1):59-65. doi:10.1093/bja/aes339

50. Toyama S, Kakumoto M, Morioka M, et al. Perfusion index derived from a pulse oximeter can predict the incidence of hypotension during spinal anaesthesia for Caesarean delivery. $\mathrm{Br}$ J Anaesth. 2013;111(2):235-241. doi:10.1093/bja/aet058

51. Bergmann I, Göhner A, Crozier TA, et al. Surgical pleth index-guided remifentanil administration reduces remifentanil and propofol consumption and shortens recovery times in outpatient anaesthesia. Br J Anaesth. 2013;110(4):622-628. doi:10.1093/bja/aes426 
52. Orbach-Zinger S, Ginosar Y, Elliston J, et al. Influence of preoperative anxiety on hypotension after spinal anaesthesia in women undergoing Caesarean delivery. Br J Anaesth. 2012;109(6):943-949. doi:10.1093/bja/aes313

53. Ryu J-H, Sohn I-S, Do S-H. Controlled hypotension for middle ear surgery: a comparison between remifentanil and magnesium sulphate. Br J Anaesth. 2009;103(4):490-495. doi:10.1093/bja/aep229

54. Trentman TL, Fassett SL, Thomas JK, Noble BN, Renfree KJ, Hattrup SJ. More hypotension in patients taking antihypertensives preoperatively during shoulder surgery in the beach chair position. Can J Anaesth. 2011;58(11):993-1000. doi:10.1007/s12630-011-9575-6

55. Visser WA, Dijkstra A, Albayrak M, Gielen MJM, Boersma E, Vonsée HJ. Spinal anesthesia for intrapartum Cesarean delivery following epidural labor analgesia: a retrospective cohort study. Can J Anaesth. 2009;56(8):577-583. doi:10.1007/s12630-009-9113-y

56. Jain K, Bhardwaj N, Sharma A, Kaur J, Kumar P. A randomised comparison of the effects of low-dose spinal or general anaesthesia on umbilical cord blood gases during caesarean delivery of growth-restricted foetuses with impaired Doppler flow. Eur J Anaesthesiol. 2013;30(1):9-15. doi:10.1097/EJA.0b013e3283564698

57. Bjørnestad E, Iversen OE, Raeder J. Wrapping of the legs versus phenylephrine for reducing hypotension in parturients having epidural anaesthesia for caesarean section: a prospective, randomized and double-blind study. Eur J Anaesthesiol. 2009;26(10):842-846. doi:10.1097/ EJA.0b013e328329b028

58. Bharti N, Sardana DK, Bala I. The Analgesic Efficacy of Dexmedetomidine as an Adjunct to Local Anesthetics in Supraclavicular Brachial Plexus Block: A Randomized Controlled Trial. Anesth Analg. 2015;121(6):1655-1660. doi:10.1213/ANE.0000000000001006

59. Panjasawatwong K, Sessler DI, Stapelfeldt WH, et al. A Randomized Trial of a Supplemental Alarm for Critically Low Systolic Blood Pressure. Anesth Analg. 2015;121(6):1500-1507. doi:10.1213/ANE.0000000000000950

60. van Waes JAR, van Klei WA, Wijeysundera DN, van Wolfswinkel L, Lindsay TF, Beattie WS. Association between Intraoperative Hypotension and Myocardial Injury after Vascular Surgery. Anesthesiology. 2016;124(1):35-44. doi:10.1097/ALN.0000000000000922

61. Sun LY, Wijeysundera DN, Tait GA, Beattie WS. Association of Intraoperative Hypotension with Acute Kidney Injury after Elective Noncardiac Surgery. Anesthesiology. 2015;123(3):515-523. doi:10.1097/ALN.0000000000000765

62. Sessler DI, Makarova N, Riveros-Perez R, Brown DL, Kimatian S. Lack of Association between Blood Pressure Management by Anesthesia Residents and Competence Committee Evaluations or In-training Exam Performance. Anesthesiology. 2016;124(2):473-482. doi:10.1097/ALN.0000000000000961

63. Levin MA, Fischer GW, Lin H-M, McCormick PJ, Krol M, Reich DL. Intraoperative arterial blood pressure lability is associated with improved 30 day survival. Br J Anaesth. 2015;115(5):716726. doi:10.1093/bja/aev293

64. Birch M, Kwon Y, Loushin MK, et al. Intrathoracic pressure regulation to treat intraoperative hypotension. Eur J Anaesthesiol. 2015;32(6):376-380. doi:10.1097/EJA.0000000000000234

65. White SM, Moppett IK, Griffiths R, et al. Secondary analysis of outcomes after 11,085 hip fracture operations from the prospective UK Anaesthesia Sprint Audit of Practice (ASAP-2). Anaesthesia. 2016;71(5):506-514. doi:10.1111/anae.13415 
66. Hsieh JK, Dalton JE, Yang D, Farag ES, Sessler DI, Kurz AM. The Association Between Mild Intraoperative Hypotension and Stroke in General Surgery Patients. Anesth Analg. 2016;123(4):933-939. doi:10.1213/ANE.0000000000001526

67. Nielsen M, Staalsoe JM, Ullum H, Secher NH, Nielsen HB, Olsen N V. The Gly16 Allele of the Gly16Arg Single-Nucleotide Polymorphism in the $\beta 2$-Adrenergic Receptor Gene Augments Perioperative Use of Vasopressors. Anesth Analg. 2016;122(5):1385-1393. doi:10.1213/ ANE.0000000000001167

68. Kamel I, Zhao H, Koch SA, Brister N, Barnette RE. The Use of Somatosensory Evoked Potentials to Determine the Relationship Between Intraoperative Arterial Blood Pressure and Intraoperative Upper Extremity Position-Related Neurapraxia in the Prone Surrender Position During Spine Surgery. Anesth Analg. 2016;122(5):1423-1433. doi:10.1213/ ANE.0000000000001121

69. Oprea AD, Lombard FW, Liu W-W, et al. Baseline Pulse Pressure, Acute Kidney Injury, and Mortality After Noncardiac Surgery. Anesth Analg. 2016;123(6):1480-1489. doi:10.1213/ ANE.0000000000001557

70. Salmasi V, Maheshwari K, Yang D, et al. Relationship between Intraoperative Hypotension, Defined by Either Reduction from Baseline or Absolute Thresholds, and Acute Kidney and Myocardial Injury after Noncardiac Surgery: A Retrospective Cohort Analysis. Anesthesiology. 2017;126(1):47-65. doi:10.1097/ALN.0000000000001432

71. McCormick PJ, Levin MA, Lin H-M, Sessler DI, Reich DL. Effectiveness of an Electronic Alert for Hypotension and Low Bispectral Index on 90-day Postoperative Mortality: A Prospective, Randomized Trial. Anesthesiology. 2016;125(6):1113-1120. doi:10.1097/ALN.0000000000001296

72. Bombardieri AM, Sharrock NE, Ma Y, Go G, Drummond JC. An Observational Study of Cerebral Blood Flow Velocity During Hypotensive Epidural Anesthesia. Anesth Analg. 2016;122(1):226233. doi:10.1213/ANE.0000000000000985

73. Yu Y, Dong J, Xu Z, Shen H, Zheng J. Pleth variability index-directed fluid management in abdominal surgery under combined general and epidural anesthesia. J Clin Monit Comput. 2015;29(1):47-52. doi:10.1007/s10877-014-9567-5

74. Zaouter C, Wehbe M, Cyr S, et al. Use of a decision support system improves the management of hemodynamic and respiratory events in orthopedic patients under propofol sedation and spinal analgesia: a randomized trial. J Clin Monit Comput. 2014;28(1):41-47. doi:10.1007/ s10877-013-9466-1

75. Brenck F, Hartmann B, Katzer C, et al. Hypotension after spinal anesthesia for cesarean section: identification of risk factors using an anesthesia information management system. J Clin Monit Comput. 2009;23(2):85-92. doi:10.1007/s10877-009-9168-x

76. Nishikawa K, Yokoyama N, Saito S, Goto F. Comparison of effects of rapid colloid loading before and after spinal anesthesia on maternal hemodynamics and neonatal outcomes in cesarean section. J Clin Monit Comput. 2007;21(2):125-129. doi:10.1007/s10877-006-9066-4

77. Sen S, Aydin K, Discigil G. Hypotension induced by lateral decubitus or supine spinal anaesthesia in elderly with low ejection fraction undergone hip surgery. J Clin Monit Comput. 2007;21(2):103-107. doi:10.1007/s10877-006-9062-8

78. Yokose M, Mihara T, Sugawara Y, Goto T. The predictive ability of non-invasive haemodynamic parameters for hypotension during caesarean section: a prospective observational study. Anaesthesia. 2015;70(5):555-562. doi:10.1111/anae.12992 
79. O'Brien C, Urquhart CS, Allam S, et al. Reaction time-monitored patient-maintained propofol sedation: a pilot study in oral surgery patients. Anaesthesia. 2013;68(7):760-764. doi:10.1111/ anae.12291

80. McCarthy T, Telec N, Dennis A, Griffiths J, Buettner A. Ability of non-invasive intermittent blood pressure monitoring and a continuous non-invasive arterial pressure monitor (CNAPTM) to provide new readings in each 1-min interval during elective caesarean section under spinal anaesthesia. Anaesthesia. 2012;67(3):274-279. doi:10.1111/j.1365-2044.2011.06996.x

81. Ghabach MB, El-Khatib MF, Zreik TG, et al. Effect of weight gain during pregnancy on heart rate variability and hypotension during caesarean section under spinal anaesthesia. Anaesthesia. 2011;66(12):1106-1111. doi:10.1111/j.1365-2044.2011.06873.x

82. Mohta M, Janani SS, Sethi AK, Agarwal D, Tyagi A. Comparison of phenylephrine hydrochloride and mephentermine sulphate for prevention of post spinal hypotension. Anaesthesia. 2010;65(12):1200-1205. doi:10.1111/j.1365-2044.2010.06559.x

83. Erdil F, Bulut S, Demirbilek S, Gedik E, Gulhas N, Ersoy MO. The effects of intrathecal levobupivacaine and bupivacaine in the elderly. Anaesthesia. 2009;64(9):942-946. doi:10.1111/ j.1365-2044.2009.05995.x

84. Lortat-Jacob B, Billard V, Buschke W, Servin F. Assessing the clinical or pharmacoeconomical benefit of target controlled desflurane delivery in surgical patients using the Zeus anaesthesia machine. Anaesthesia. 2009;64(11):1229-1235. doi:10.1111/j.13652044.2009.06081.x

85. Cuvas O, Gulec H, Karaaslan M, Basar H. The use of low dose plain solutions of local anaesthetic agents for spinal anaesthesia in the prone position: bupivacaine compared with levobupivacaine. Anaesthesia. 2009;64(1):14-18. doi:10.1111/j.1365-2044.2008.05680.x

86. Rueggeberg A, Boehm S, Napieralski F, et al. Development of a risk stratification model for predicting acute renal failure in orthotopic liver transplantation recipients. Anaesthesia. 2008;63(11):1174-1180. doi:10.1111/j.1365-2044.2008.05604.x

87. Kancir ASP, Johansen JK, Ekeloef NP, Pedersen EB. The Effect of 6\% Hydroxyethyl Starch 130/0.4 on Renal Function, Arterial Blood Pressure, and Vasoactive Hormones During Radical Prostatectomy. Anesth Analg. 2015;120(3):608-618. doi:10.1213/ANE.0000000000000596

88. Ladha KS, Nanji KC, Pierce E, Poon KT, Hyder JA. The Impact of a Shortage of PharmacyPrepared Ephedrine Syringes on Intraoperative Medication Use. Anesth Analg. June 2015. doi:10.1213/ANE.0000000000000809

89. Laflam A, Joshi B, Brady K, et al. Shoulder surgery in the beach chair position is associated with diminished cerebral autoregulation but no differences in postoperative cognition or brain injury biomarker levels compared with supine positioning: the anesthesia patient safety foundation be. Anesth Analg. 2015;120(1):176-185. doi:10.1213/ANE.0000000000000455

90. Turan A, Hesler BD, You J, et al. The association of serum vitamin D concentration with serious complications after noncardiac surgery. Anesth Analg. 2014;119(3):603-612. doi:10.1213/ ANE.0000000000000096

91. Pestaña D, Espinosa E, Eden A, et al. Perioperative goal-directed hemodynamic optimization using noninvasive cardiac output monitoring in major abdominal surgery: a prospective, randomized, multicenter, pragmatic trial: POEMAS Study (PeriOperative goal-directed thErapy in Major Abdominal Surg. Anesth Analg. 2014;119(3):579-587. doi:10.1213/ ANE.0000000000000295 
92. Lee J, Lee J, Ko S. The relationship between serum progesterone concentration and anesthetic and analgesic requirements: a prospective observational study of parturients undergoing cesarean delivery. Anesth Analg. 2014;119(4):901-905. doi:10.1213/ ANE.0000000000000366

93. Chen M, Chen C, Ke Q. The effect of age on the median effective dose (ED50) of intrathecally administered plain bupivacaine for motor block. Anesth Analg. 2014;118(4):863-868. doi:10.1213/ANE.0000000000000147

94. Lilot M, Meuret P, Bouvet L, et al. Hypobaric spinal anesthesia with ropivacaine plus sufentanil for traumatic femoral neck surgery in the elderly: a dose-response study. Anesth Analg. 2013;117(1):259-264. doi:10.1213/ANE.0b013e31828f29f8

95. Allyn J, Guglielminotti J, Omnes S, et al. Marfan's syndrome during pregnancy: anesthetic management of delivery in 16 consecutive patients. Anesth Analg. 2013;116(2):392-398. doi:10.1213/ANE.0b013e3182768f78

96. Doherty A, Ohashi Y, Downey K, Carvalho JCA. Phenylephrine infusion versus bolus regimens during cesarean delivery under spinal anesthesia: a double-blind randomized clinical trial to assess hemodynamic changes. Anesth Analg. 2012;115(6):1343-1350. doi:10.1213/ ANE.0b013e31826ac3db

97. Loubert C, Hallworth S, Fernando R, et al. Does the baricity of bupivacaine influence intrathecal spread in the prolonged sitting position before elective cesarean delivery? A prospective randomized controlled study. Anesth Analg. 2011;113(4):811-817. doi:10.1213/ ANE.0b013e3182288bf2

98. McDonald S, Fernando R, Ashpole K, Columb M. Maternal Cardiac Output Changes After Crystalloid or Colloid Coload Following Spinal Anesthesia for Elective Cesarean Delivery. Anesth Analg. 2011;113(4):1. doi:10.1213/ANE.0b013e31822c0f08

99. Osugi T, Tatara T, Yada S, Tashiro C. Hydration status after overnight fasting as measured by urine osmolality does not alter the magnitude of hypotension during general anesthesia in low risk patients. Anesth Analg. 2011;112(6):1307-1313. doi:10.1213/ANE.0b013e3182114df4

100. Song JW, Lee Y-W, Yoon KB, Park SJ, Shim YH. Magnesium sulfate prevents remifentanilinduced postoperative hyperalgesia in patients undergoing thyroidectomy. Anesth Analg. 2011;113(2):390-397. doi:10.1213/ANE.0b013e31821d72bc

101. Jeon Y-T, Hwang J-W, Kim M-H, et al. Positional blood pressure change and the risk of hypotension during spinal anesthesia for cesarean delivery: an observational study. Anesth Analg. 2010;111(3):712-715. doi:10.1213/ANE.0b013e3181e8137b

102. Patel A, Davidson M, Tran MCJ, et al. Dexmedetomidine infusion for analgesia and prevention of emergence agitation in children with obstructive sleep apnea syndrome undergoing tonsillectomy and adenoidectomy. Anesth Analg. 2010;111(4):1004-1010. doi:10.1213/ ANE.0b013e3181ee82fa

103. Forget P, Lois F, de Kock M. Goal-directed fluid management based on the pulse oximeterderived pleth variability index reduces lactate levels and improves fluid management. Anesth Analg. 2010;111(4):910-914. doi:10.1213/ANE.0b013e3181eb624f

104. George RB, McKeen D, Columb MO, Habib AS. Up-down determination of the $90 \%$ effective dose of phenylephrine for the treatment of spinal anesthesia-induced hypotension in parturients undergoing cesarean delivery. Anesth Analg. 2010;110(1):154-158. doi:10.1213/ ANE.0b013e3181c30b72 
105. Candiotti KA, Bergese SD, Bokesch PM, Feldman MA, Wisemandle W, Bekker AY. Monitored anesthesia care with dexmedetomidine: a prospective, randomized, double-blind, multicenter trial. Anesth Analg. 2010;110(1):47-56. doi:10.1213/ane.0b013e3181ae0856

106. Esmaoglu A, Yegenoglu F, Akin A, Turk CY. Dexmedetomidine added to levobupivacaine prolongs axillary brachial plexus block. Anesth Analg. 2010;111(6):1548-1551. doi:10.1213/ ANE.0b013e3181fa3095

107. Siddik-Sayyid SM, Nasr VG, Taha SK, et al. A randomized trial comparing colloid preload to coload during spinal anesthesia for elective cesarean delivery. Anesth Analg. 2009;109(4):12191224. doi:10.1213/ane.0b013e3181b2bd6b

108. Leo S, Sng BL, Lim Y, Sia ATH. A randomized comparison of low doses of hyperbaric bupivacaine in combined spinal-epidural anesthesia for cesarean delivery. Anesth Analg. 2009;109(5):1600-1605. doi:10.1213/ANE.0b013e3181b72d35

109. Panousis P, Heller AR, Koch T, Litz RJ. Epidural ropivacaine concentrations for intraoperative analgesia during major upper abdominal surgery: a prospective, randomized, doubleblinded, placebo-controlled study. Anesth Analg. 2009;108(6):1971-1976. doi:10.1213/ ane.0b013e3181a2a301

110. Zorko N, Kamenik M, Starc V. The effect of Trendelenburg position, lactated Ringer's solution and 6\% hydroxyethyl starch solution on cardiac output after spinal anesthesia. Anesth Analg. 2009;108(2):655-659. doi:10.1213/ane.0b013e31818ec9e5

111. Tamilselvan P, Fernando R, Bray J, Sodhi M, Columb M. The effects of crystalloid and colloid preload on cardiac output in the parturient undergoing planned cesarean delivery under spinal anesthesia: a randomized trial. Anesth Analg. 2009;109(6):1916-1921. doi:10.1213/ ANE.0b013e3181bbfdf6

112. Kungys G, Rose DD, Fleming NW. Stroke volume variation during acute normovolemic hemodilution. Anesth Analg. 2009;109(6):1823-1830. doi:10.1213/ANE.0b013e3181ba41af

113. Ross VH, Pan PH, Owen MD, et al. Neostigmine decreases bupivacaine use by patientcontrolled epidural analgesia during labor: a randomized controlled study. Anesth Analg. 2009;109(2):524-531. doi:10.1213/ane.0b013e31819518e4

114. Minville V, Asehnoune K, Salau S, et al. The effects of spinal anesthesia on cerebral blood flow in the very elderly. Anesth Analg. 2009;108(4):1291-1294. doi:10.1213/ane.0b013e31819b073b

115. Bhagat H, Dash HH, Bithal PK, Chouhan RS, Pandia MP. Planning for early emergence in neurosurgical patients: a randomized prospective trial of low-dose anesthetics. Anesth Analg. 2008;107(4):1348-1355. doi:10.1213/ane.0b013e31817f9476

116. Li Y, Zhu S, Yan M. Combined general/epidural anesthesia (ropivacaine 0.375\%) versus general anesthesia for upper abdominal surgery. Anesth Analg. 2008;106(5):1562-5, table of contents. doi:10.1213/ane.0b013e31816d1976

117. Tufanogullari B, White PF, Peixoto MP, et al. Dexmedetomidine infusion during laparoscopic bariatric surgery: the effect on recovery outcome variables. Anesth Analg. 2008;106(6):17411748. doi:10.1213/ane.0b013e318172c47c

118. Szmuk P, Aroyo N, Ezri T, Muzikant G, Weisenberg M, Sessler DI. Listening to music during anesthesia does not reduce the sevoflurane concentration needed to maintain a constant bispectral index. Anesth Analg. 2008;107(1):77-80. doi:10.1213/ane.0b013e3181733e16 
119. Koç S, Memis D, Sut N. The preoperative use of gabapentin, dexamethasone, and their combination in varicocele surgery: a randomized controlled trial. Anesth Analg. 2007;105(4):1137-42, table of contents. doi:10.1213/01.ane.0000278869.00918.b7

120. Balki M, Kasodekar S, Dhumne S, Carvalho JCA. Prophylactic [corrected] granisetron does not prevent postdelivery nausea and vomiting during elective cesarean delivery under spinal anesthesia. Anesth Analg. 2007;104(3):679-683. doi:10.1213/01.ane.0000253036.06307.5c

121. Zohar E, Noga Y, Rislick U, Leibovitch I, Fredman B. Intrathecal anesthesia for elderly patients undergoing short transurethral procedures: a dose-finding study. Anesth Analg. 2007;104(3):552-554. doi:10.1213/01.ane.0000255329.55037.cd

122. Kaabachi O, Zarghouni A, Ouezini R, Abdelaziz A Ben, Chattaoui O, Kokki H. Clonidine 1 microg/kg is a safe and effective adjuvant to plain bupivacaine in spinal anesthesia in adolescents. Anesth Analg. 2007;105(2):516-519. doi:10.1213/01.ane.0000268709.67572.09

123. Ngan Kee WD, Khaw KS, Ng FF, Karmakar MK, Critchley LA, Gin T. A prospective comparison of vasopressor requirement and hemodynamic changes during spinal anesthesia for cesarean delivery in patients with multiple gestation versus singleton pregnancy. Anesth Analg. 2007;104(2):407-411. doi:10.1213/01.ane.0000252461.97656.3e

124. Kancir ASP, Pleckaitiene L, Hansen TB, Ekeløf NP, Pedersen EB. Lack of nephrotoxicity by 6\% hydroxyethyl starch 130/0.4 during hip arthroplasty: a randomized controlled trial. Anesthesiology. 2014;121(5):948-958. doi:10.1097/ALN.0000000000000413

125. El Hor T, Van Der Linden P, De Hert S, Mélot C, Bidgoli J. Impact of entropy monitoring on volatile anesthetic uptake. Anesthesiology. 2013;118(4):868-873. doi:10.1097/ ALN.0b013e3182850c36

126. Jeong H, Jeong S, Lim HJ, Lee J, Yoo KY. Cerebral oxygen saturation measured by near-infrared spectroscopy and jugular venous bulb oxygen saturation during arthroscopic shoulder surgery in beach chair position under sevoflurane-nitrous oxide or propofol-remifentanil anesthesia. Anesthesiology. 2012;116(5):1047-1056. doi:10.1097/ALN.0b013e31825154d2

127. Davis MJ, Menon BK, Baghirzada LB, et al. Anesthetic management and outcome in patients during endovascular therapy for acute stroke. Anesthesiology. 2012;116(2):396-405. doi:10.1097/ALN.0b013e318242a5d2

128. Wax DB, Lin H-M, Leibowitz AB. Invasive and concomitant noninvasive intraoperative blood pressure monitoring: observed differences in measurements and associated therapeutic interventions. Anesthesiology. 2011;115(5):973-978. doi:10.1097/ALN.0b013e3182330286

129. Ngan Kee WD, Khaw KS, Tan PE, Ng FF, Karmakar MK. Placental transfer and fetal metabolic effects of phenylephrine and ephedrine during spinal anesthesia for cesarean delivery. Anesthesiology. 2009;111(3):506-512. doi:10.1097/ALN.0b013e3181b160a3

130. Kheterpal S, O'Reilly M, Englesbe MJ, et al. Preoperative and intraoperative predictors of cardiac adverse events after general, vascular, and urological surgery. Anesthesiology. 2009;110(1):58-66. doi:10.1097/ALN.0b013e318190b6dc

131. Dyer RA, Piercy JL, Reed AR, Lombard CJ, Schoeman LK, James MF. Hemodynamic changes associated with spinal anesthesia for cesarean delivery in severe preeclampsia. Anesthesiology. 2008;108(5):802-811. doi:10.1097/01.anes.0000311153.84687.c7 
132. Wappler F, Rossaint R, Baumert J, et al. Multicenter randomized comparison of xenon and isoflurane on left ventricular function in patients undergoing elective surgery. Anesthesiology. 2007;106(3):463-471. http://www.ncbi.nlm.nih.gov/pubmed/17325504. Accessed April 23, 2015.

133. MacDonald N, Ahmad T, Mohr O, et al. Dynamic preload markers to predict fluid responsiveness during and after major gastrointestinal surgery: an observational substudy of the OPTIMISE trial. Br J Anaesth. 2015;114(4):598-604. doi:10.1093/bja/aeu398

134. Leslie K, Myles P, Devereaux P, et al. Neuraxial block, death and serious cardiovascular morbidity in the POISE trial. Br J Anaesth. 2013;111(3):382-390. doi:10.1093/bja/aet120

135. AbdelmalakBB, Cata JP, Bonilla A, et al. Intraoperative tissue oxygenation and postoperative outcomes after major non-cardiac surgery: an observational study. Br J Anaesth. 2013;110(2):241-249. doi:10.1093/bja/aes378

136. Kim SY, Kim JM, Lee JH, Song BM, Koo BN. Efficacy of intraoperative dexmedetomidine infusion on emergence agitation and quality of recovery after nasal surgery. Br J Anaesth. 2013;111(2):222-228. doi:10.1093/bja/aet056

137. Soeding PF, Hoy S, Hoy G, Evans M, Royse CF. Effect of phenylephrine on the haemodynamic state and cerebral oxygen saturation during anaesthesia in the upright position. Br J Anaesth. 2013;111(2):229-234. doi:10.1093/bja/aet024

138. Kim WH, Lee JJ, Lee SM, et al. Comparison of motor-evoked potentials monitoring in response to transcranial electrical stimulation in subjects undergoing neurosurgery with partial vs no neuromuscular block. Br J Anaesth. 2013;110(4):567-576. doi:10.1093/bja/aes395

139. Bartha E, Arfwedson C, Imnell A, Fernlund ME, Andersson LE, Kalman S. Randomized controlled trial of goal-directed haemodynamic treatment in patients with proximal femoral fracture. Br J Anaesth. 2013;110(4):545-553. doi:10.1093/bja/aes468

140. Ilies C, Kiskalt H, Siedenhans D, et al. Detection of hypotension during Caesarean section with continuous non-invasive arterial pressure device or intermittent oscillometric arterial pressure measurement. Br J Anaesth. 2012;109(3):413-419. doi:10.1093/bja/aes224

141. Park BY, Jeong CW, Jang EA, et al. Dose-related attenuation of cardiovascular responses to tracheal intubation by intravenous remifentanil bolus in severe pre-eclamptic patients undergoing Caesarean delivery. Br J Anaesth. 2011;106(1):82-87. doi:10.1093/bja/aeq275

142. Sabaté S, Mases A, Guilera N, et al. Incidence and predictors of major perioperative adverse cardiac and cerebrovascular events in non-cardiac surgery. Br J Anaesth. 2011;107(6):879-890. doi:10.1093/bja/aer268

143. Butwick AJ, Coleman L, Cohen SE, Riley ET, Carvalho B. Minimum effective bolus dose of oxytocin during elective Caesarean delivery. Br J Anaesth. 2010;104(3):338-343. doi:10.1093/ bja/aeq004

144. Hwang J-Y, Na H-S, Jeon Y-T, Ro Y-J, Kim C-S, Do S-H. I.V. infusion of magnesium sulphate during spinal anaesthesia improves postoperative analgesia. Br J Anaesth. 2010;104(1):89-93. doi:10.1093/bja/aep334

145. Hennebry MC, Stocks GM, Belavadi P, et al. Effect of i.v. phenylephrine or ephedrine on the ED50 of intrathecal bupivacaine with fentanyl for caesarean section. Br J Anaesth. 2009;102(6):806-811. doi:10.1093/bja/aep095 
146. Baumert J-H, Hein M, Hecker KE, Satlow S, Neef P, Rossaint R. Xenon or propofol anaesthesia for patients at cardiovascular risk in non-cardiac surgery. Br J Anaesth. 2008;100(5):605-611. doi:10.1093/bja/aen050

147. Ryu J-H, Kang M-H, Park K-S, Do S-H. Effects of magnesium sulphate on intraoperative anaesthetic requirements and postoperative analgesia in gynaecology patients receiving total intravenous anaesthesia. Br J Anaesth. 2008;100(3):397-403. doi:10.1093/bja/aem407

148. Arai YCP, Kato N, Matsura M, et al. Transcutaneous electrical nerve stimulation at the PC-5 and PC- 6 acupoints reduced the severity of hypotension after spinal anaesthesia in patients undergoing Caesarean section. Br J Anaesth. 2008;100(1):78-81. doi:10.1093/bja/aem306

149. De Baerdemaeker LEC, Jacobs S, Pattyn P, Mortier EP, Struys MMRF. Influence of intraoperative opioid on postoperative pain and pulmonary function after laparoscopic gastric banding: remifentanil TCI vs sufentanil TCl in morbid obesity. Br J Anaesth. 2007;99(3):404-411. doi:10.1093/bja/aem164

150. Kim J-T, Shim J-K, Kim S-H, Jung C-W, Bahk J-H. Trendelenburg position with hip flexion as a rescue strategy to increase spinal anaesthetic level after spinal block. Br J Anaesth. 2007;98(3):396-400. doi:10.1093/bja/ael370

151. Nguyen-Lu N, Carvalho JCA, Farine D, Seaward G, Ye XY, Balki M. Carbetocin at Cesarean delivery for labour arrest: a sequential allocation trial to determine the effective dose. Can J Anaesth. April 2015. doi:10.1007/s12630-015-0375-2

152. Moore A, Bourrassa-Blanchette S, El Mouallem E, et al. The median effective seated time for hypotension induced by spinal anesthesia at Cesarean delivery with two doses of hyperbaric bupivacaine: a randomized up-down sequential allocation study. Can J Anaesth. 2014;61(10):916-921. doi:10.1007/s12630-014-0208-8

153. Khan M, Balki M, Ahmed I, Farine D, Seaward G, Carvalho JCA. Carbetocin at elective Cesarean delivery: a sequential allocation trial to determine the minimum effective dose. Can J Anaesth. 2014;61(3):242-248. doi:10.1007/s12630-013-0082-9

154. Hwang J, Min S, Kim C, Gil N, Kim E, Huh J. Prophylactic glycopyrrolate reduces hypotensive responses in elderly patients during spinal anesthesia: a randomized controlled trial. Can J Anaesth. 2014;61(1):32-38. doi:10.1007/s12630-013-0064-y

155. Anandakrishnan S, Balki M, Farine D, Seaward G, Carvalho JCA. Carbetocin at elective Cesarean delivery: a randomized controlled trial to determine the effective dose, part 2. Can J Anaesth. 2013;60(11):1054-1060. doi:10.1007/s12630-013-0028-2

156. Cordovani D, Balki M, Farine D, Seaward G, Carvalho JCA. Carbetocin at elective Cesarean delivery: a randomized controlled trial to determine the effective dose. Can J Anaesth. 2012;59(8):751-757. doi:10.1007/s12630-012-9728-2

157. Yadeau JT, Liu SS, Bang H, et al. Cerebral oximetry desaturation during shoulder surgery performed in a sitting position under regional anesthesia. Can J Anaesth. 2011;58(11):986992. doi:10.1007/s12630-011-9574-7

158. El-Hakeem EEA, Kaki AM, Almazrooa AA, Al-Mansouri NM, Alhashemi JA. Effects of sitting up for five minutes versus immediately lying down after spinal anesthesia for Cesarean delivery on fluid and ephedrine requirement; a randomized trial. Can J Anaesth. 2011;58(12):10831089. doi:10.1007/s12630-011-9593-4

159. Istvan J, Belliveau M, Donati F. Rapid sequence induction for appendectomies: a retrospective case-review analysis. Can J Anaesth. 2010;57(4):330-336. doi:10.1007/s12630-009-9260-1 
160. Lauwick S, Kim DJ, Michelagnoli G, et al. Intraoperative infusion of lidocaine reduces postoperative fentanyl requirements in patients undergoing laparoscopic cholecystectomy. Can J Anaesth. 2008;55(11):754-760. http://www.ncbi.nlm.nih.gov/pubmed/19138915. Accessed July 10, 2015.

161. Danelli G, Baciarello M, Di Cianni S, et al. Effects of baricity of $0.5 \%$ or $0.75 \%$ levobupivacaine on the onset time of spinal anesthesia: a randomized trial. Can J Anaesth. 2008;55(8):501-506. doi:10.1007/BF03016669

162. Bryson GL, Macneil R, Jeyaraj LM, Rosaeg OP. Small dose spinal bupivacaine for Cesarean delivery does not reduce hypotension but accelerates motor recovery. Can J Anaesth. 2007;54(7):531-537. doi:10.1007/BF03022316

163. Singh SI, Morley-Forster PK, Shamsah M, Butler R. Influence of injection rate of hyperbaric bupivacaine on spinal block in parturients: a randomized trial. Can J Anaesth. 2007;54(4):290295. doi:10.1007/BF03022774

164. Moerman AT, De Hert SG, Jacobs TF, De Wilde LF, Wouters PF. Cerebral oxygen desaturation during beach chair position. Eur J Anaesthesiol. 2012;29(2):82-87. doi:10.1097/ EJA.0b013e328348ca18

165. Khan ZH, Zanjani AP, Makarem J, Samadi S. Antishivering effects of two different doses of intrathecal meperidine in caesarean section: a prospective randomised blinded study. Eur J Anaesthesiol. 2011;28(3):202-206. doi:10.1097/EJA.0b013e3283430802

166. Jildenstål PK, Hallén JL, Rawal N, Gupta A, Berggren L. Effect of auditory evoked potentialguided anaesthesia on consumption of anaesthetics and early postoperative cognitive dysfunction: a randomised controlled trial. Eur J Anaesthesiol. 2011;28(3):213-219. doi:10.1097/EJA.0b013e328340dbb9

167. Yoo KY, Kim TS, Jeong CW, et al. Anaesthetic requirements and stress hormone responses in acute cord-injured patients undergoing surgery of the injured spine. Eur J Anaesthesiol. 2009;26(4):304-310. doi:10.1097/EJA.0b013e328326e035

168. Smith I, Walley G, Bridgman S. Omitting fentanyl reduces nausea and vomiting, without increasing pain, after sevoflurane for day surgery. Eur J Anaesthesiol. 2008;25(10):790-799. doi:10.1017/S026502150800464X

169. Choi S-S, Jun I-G, Cho S-S, Kim S-K, Hwang G-S, Kim Y-K. Effect of stroke volume variationdirected fluid management on blood loss during living-donor right hepatectomy: a randomised controlled study. Anaesthesia. 2015;70(11):1250-1258. doi:10.1111/anae.13155

170. Pedoto A, Desiderio DP, Amar D, Downey RJ. Hemodynamic Instability Following Airway Spray Cryotherapy. Anesth Analg. 2016;123(5):1302-1306. http://www.ncbi.nlm.nih.gov/ pubmed/27763916. Accessed March 21, 2019.

171. Abdulatif M, Ahmed A, Mukhtar A, Badawy S. The effect of magnesium sulphate infusion on the incidence and severity of emergence agitation in children undergoing adenotonsillectomy using sevoflurane anaesthesia. Anaesthesia. 2013;68(10):1045-1052. doi:10.1111/anae.12380

172. Abbott TEF, Pearse RM, Archbold RA, et al. A Prospective International Multicentre Cohort Study of Intraoperative Heart Rate and Systolic Blood Pressure and Myocardial Injury After Noncardiac Surgery. Anesth Analg. 2018;126(6):1936-1945. doi:10.1213/ ANE.0000000000002560 
173. Ceruti S, Anselmi L, Minotti B, et al. Prevention of arterial hypotension after spinal anaesthesia using vena cava ultrasound to guide fluid management. Br J Anaesth. 2018;120(1):101-108. doi:10.1016/j.bja.2017.08.001

174. Calvo-Vecino JM, Ripollés-Melchor J, Mythen MG, et al. Effect of goal-directed haemodynamic therapy on postoperative complications in low-moderate risk surgical patients: a multicentre randomised controlled trial (FEDORA trial). Br J Anaesth. 2018;120(4):734-744. doi:10.1016/j. bja.2017.12.018

175. Bishop DG, Cairns C, Grobbelaar M, Rodseth RN. Prophylactic Phenylephrine Infusions to Reduce Severe Spinal Anesthesia Hypotension During Cesarean Delivery in a Resource-Constrained Environment. Anesth Analg. 2017;125(3):904-906. doi:10.1213/ ANE.0000000000001905

176. Aouad MT, Zeeni C, Al Nawwar R, et al. Dexmedetomidine for Improved Quality of Emergence From General Anesthesia. Anesth Analg. December 2017:1. doi:10.1213/ ANE.0000000000002763

177. Duffield A, McKenzie C, Carvalho B, et al. Effect of a High-Rate Versus a Low-Rate Oxytocin Infusion for Maintaining Uterine Contractility During Elective Cesarean Delivery. Anesth Analg. 2017;124(3):857-862. doi:10.1213/ANE.0000000000001658

178. Du CH, Glick D, Tung A. Error-checking intraoperative arterial line blood pressures. J Clin Monit Comput. June 2018. doi:10.1007/s10877-018-0167-7

179. Crowther M, van der Spuy K, Roodt F, et al. The relationship between pre-operative hypertension and intra-operative haemodynamic changes known to be associated with postoperative morbidity. Anaesthesia. 2018;73(7):812-818. doi:10.1111/anae.14239

180. Roshanov PS, Rochwerg B, Patel A, et al. Withholding versus Continuing Angiotensinconverting Enzyme Inhibitors or Angiotensin II Receptor Blockers before Noncardiac Surgery. Anesthesiology. 2017;126(1):16-27. doi:10.1097/ALN.0000000000001404

181. Chang J-E, Kim H, Ryu J-H, Lee J-M, Hwang J-Y. Relationship Between Central Obesity and Spread of Spinal Anesthesia in Female Patients. Anesth Analg. 2017;124(5):1670-1673. doi:10.1213/ANE.0000000000001817

182. Clemmesen CG, Pedersen LM, Hougaard S, et al. Cerebral oximetry during preoperative resuscitation in elderly patients with hip fracture: a prospective observational study. J Clin Monit Comput. 2018;32(6):1033-1040. doi:10.1007/s10877-018-0107-6

183. Kheterpal S, Shanks A, Tremper KK. Impact of a Novel Multiparameter Decision Support System on Intraoperative Processes of Care and Postoperative Outcomes. Anesthesiology. 2018;128(2):272-282. doi:10.1097/ALN.0000000000002023

184. Kober D, Trepte C, Petzoldt M, et al. Cardiac index assessment using bioreactance in patients undergoing cytoreductive surgery in ovarian carcinoma. J Clin Monit Comput. 2013;27(6):621627. doi:10.1007/s10877-013-9478-x

185. George RB, McKeen DM, Dominguez JE, Allen TK, Doyle PA, Habib AS. A randomized trial of phenylephrine infusion versus bolus dosing for nausea and vomiting during Cesarean delivery in obese women. Can J Anaesth. 2018;65(3):254-262. doi:10.1007/s12630-017-1034-6

186. Dumans-Nizard V, Le Guen M, Sage E, Chazot T, Fischler M, Liu N. Thoracic Epidural Analgesia With Levobupivacaine Reduces Remifentanil and Propofol Consumption Evaluated by Closed-Loop Titration Guided by the Bispectral Index. Anesth Analg. 2017;125(2):635-642. doi:10.1213/ANE.0000000000001996 
187. Jakobsson J, Kalman SH, Lindeberg-Lindvet M, Bartha E. Is postspinal hypotension a sign of impaired cardiac performance in the elderly? An observational mechanistic study. $\mathrm{Br} \mathrm{J}$ Anaesth. 2017;119(6):1178-1185. doi:10.1093/bja/aex274

188. Jacka MJ, Guyatt G, Mizera R, et al. Age Does Not Affect Metoprolol's Effect on Perioperative Outcomes (From the POISE Database). Anesth Analg. 2018;126(4):1150-1157. doi:10.1213/ ANE.0000000000002804

189. Katz D, Hamburger J, Gutman D, et al. The Effect of Adding Subarachnoid Epinephrine to Hyperbaric Bupivacaine and Morphine for Repeat Cesarean Delivery. Anesth Analg. 2018;127(1):171-178. doi:10.1213/ANE.0000000000002542

190. Juri T, Suehiro K, Kimura A, et al. Impact of continuous non-invasive blood pressure monitoring on hemodynamic fluctuation during general anesthesia: a randomized controlled study. J Clin Monit Comput. 2018;32(6):1005-1013. doi:10.1007/s10877-018-0125-4

191. Ji F, Li J, Fleming N, Rose D, Liu H. Reliability of a new 4th generation FloTrac algorithm to track cardiac output changes in patients receiving phenylephrine. J Clin Monit Comput. September 2014. doi:10.1007/s10877-014-9624-0

192. Hallqvist L, Granath F, Huldt E, Bell M. Intraoperative hypotension is associated with acute kidney injury in noncardiac surgery. Eur J Anaesthesiol. 2018;35(4):273-279. doi:10.1097/ EJA.0000000000000735

193. Kruger GH, Shanks A, Kheterpal S, et al. Influence of non-invasive blood pressure measurement intervals on the occurrence of intra-operative hypotension. J Clin Monit Comput. 2018;32(4):699-705. doi:10.1007/s10877-017-0065-4

194. Kuhn JC, Hauge TH, Rosseland LA, DahI V, Langesæeter E. Hemodynamics of Phenylephrine Infusion Versus Lower Extremity Compression During Spinal Anesthesia for Cesarean Delivery. Anesth Analg. 2016;122(4):1120-1129. doi:10.1213/ANE.0000000000001174

195. Ismail SA, Mowafi HA. Melatonin provides anxiolysis, enhances analgesia, decreases intraocular pressure, and promotes better operating conditions during cataract surgery under topical anesthesia. Anesth Analg. 2009;108(4):1146-1151. doi:10.1213/ ane.0b013e3181907ebe

196. Maheshwari K, Khanna S, Bajracharya GR, et al. A Randomized Trial of Continuous Noninvasive Blood Pressure Monitoring During Noncardiac Surgery. Anesth Analg. 2018;127(2):424-431. doi:10.1213/ANE.0000000000003482

197. Leblanc D, Conté M, Masson G, et al. SmartPilot ${ }^{\circledR}$ view-guided anaesthesia improves postoperative outcomes in hip fracture surgery: a randomized blinded controlled study. $\mathrm{Br}$ J Anaesth. 2017;119(5):1022-1029. doi:10.1093/bja/aex317

198. Meidert AS, Nold JS, Hornung R, Paulus AC, Zwißler B, Czerner S. The impact of continuous non-invasive arterial blood pressure monitoring on blood pressure stability during general anaesthesia in orthopaedic patients. Eur J Anaesthesiol. 2017;34(11):716-722. doi:10.1097/ EJA.0000000000000690

199. McCann ME, Withington DE, Arnup SJ, et al. Differences in Blood Pressure in Infants After General Anesthesia Compared to Awake Regional Anesthesia (GAS Study-A Prospective Randomized Trial). Anesth Analg. 2017;125(3):837-845. doi:10.1213/ANE.0000000000001870

200. Mathis MR, Sathishkumar S, Kheterpal S, et al. Complications, Risk Factors, and Staffing Patterns for Noncardiac Surgery in Patients with Left Ventricular Assist Devices. Anesthesiology. 2017;126(3):450-460. doi:10.1097/ALN.0000000000001488 
201. Mathis MR, Schechtman SA, Engoren MC, et al. Arterial Pressure Variation in Elective Noncardiac Surgery. Anesthesiology. 2017;126(2):249-259. doi:10.1097/ALN.0000000000001460

202. Ngan Kee WD, Khaw KS, Tam Y-H, Ng FF, Lee SW. Performance of a closed-loop feedback computer-controlled infusion system for maintaining blood pressure during spinal anaesthesia for caesarean section: a randomized controlled comparison of norepinephrine versus phenylephrine. J Clin Monit Comput. 2017;31(3):617-623. doi:10.1007/s10877-016-9883-z

203. Ngan Kee WD. A Random-allocation Graded Dose-Response Study of Norepinephrine and Phenylephrine for Treating Hypotension during Spinal Anesthesia for Cesarean Delivery. Anesthesiology. 2017;127(6):934-941. doi:10.1097/ALN.0000000000001880

204. Ngan Kee WD, Tam Y-H, Khaw KS, Ng FF, Lee SWY. Closed-Loop Feedback ComputerControlled Phenylephrine for Maintenance of Blood Pressure During Spinal Anesthesia for Cesarean Delivery. Anesth Analg. 2017;125(1):117-123. doi:10.1213/ANE.0000000000001974

205. Moore A, el-Mouallem E, el-Bahrawy A, et al. An up-down determination of the required seated duration after intrathecal injection of bupivacaine and fentanyl for the prevention of hypotension during Cesarean delivery. Can J Anesth Can d'anesthésie. 2017;64(10):1002-1008. doi:10.1007/s12630-017-0931-z

206. Nguyen HP, Zaroff JG, Bayman EO, Gelb AW, Todd MM, Hindman BJ. Perioperative hypothermia (33 degrees $\mathrm{C}$ ) does not increase the occurrence of cardiovascular events in patients undergoing cerebral aneurysm surgery: findings from the Intraoperative Hypothermia for Aneurysm Surgery Trial. Anesthesiology. 2010;113(2):327-342. doi:10.1097/ ALN.0b013e3181dfd4f7

207. Olbrecht VA, Skowno J, Marchesini V, et al. An International, Multicenter, Observational Study of Cerebral Oxygenation during Infant and Neonatal Anesthesia. Anesthesiology. 2018;128(1):85-96. doi:10.1097/ALN.0000000000001920

208. Ottaway AJ, Kabongo L. Prospective Observational Study of Intraoperative Anesthetic Events in District Hospitals in Namibia. Anesth Analg. 2018;126(2):632-638. doi:10.1213/ ANE.0000000000002695

209. Onwochei DN, Ngan Kee WD, Fung L, Downey K, Ye XY, Carvalho JCA. Norepinephrine Intermittent Intravenous Boluses to Prevent Hypotension During Spinal Anesthesia for Cesarean Delivery. Anesth Analg. 2017;125(1):212-218. doi:10.1213/ANE.0000000000001846

210. Olesen ND, Sørensen H, Ambrus R, Svendsen LB, Lund A, Secher NH. A mesenteric traction syndrome affects near-infrared spectroscopy evaluated cerebral oxygenation because skin blood flow increases. J Clin Monit Comput. 2018;32(2):261-268. doi:10.1007/s10877-017-0014-2

211. Sessler DI, Meyhoff CS, Zimmerman NM, et al. Period-dependent Associations between Hypotension during and for Four Days after Noncardiac Surgery and a Composite of Myocardial Infarction and Death. Anesthesiology. 2018;128(2):317-327. doi:10.1097/ ALN.0000000000001985

212. Prashanth A, Chakravarthy M, George A, Mayur R, Hosur R, Pargaonkar S. Sympatho-vagal balance, as quantified by ANSindex, predicts post spinal hypotension and vasopressor requirement in parturients undergoing lower segmental cesarean section: a single blinded prospective observational study. J Clin Monit Comput. 2017;31(4):805-811. doi:10.1007/s10877016-9906-9 
213. Pfortmueller CA, Funk G-C, Reiterer C, et al. Normal saline versus a balanced crystalloid for goal-directed perioperative fluid therapy in major abdominal surgery: a double-blind randomised controlled study. Br J Anaesth. 2018;120(2):274-283. doi:10.1016/j.bja.2017.11.088

214. Pace MM, Sharma B, Anderson-Dam J, Fleischmann K, Warren L, Stefanovich P. UltrasoundGuided Thoracic Paravertebral Blockade. Anesth Analg. 2016;122(4):1186-1191. doi:10.1213/ ANE.0000000000001117

215. Stapelfeldt WH, Yuan H, Dryden JK, et al. The SLUScore. Anesth Analg. 2017;124(4):1135-1152. doi:10.1213/ANE.0000000000001797

216. Shah S, Ross O, Pickering S, Knoble S, Rai I. Tablet e-Logbooks. Anesth Analg. 2017;125(4):13371341. doi:10.1213/ANE.0000000000002094

217. Turan A, You J, Egan C, et al. Relationship between Chronic Intermittent Hypoxia and Intraoperative Mean Arterial Pressure in Obstructive Sleep Apnea Patients Having Laparoscopic Bariatric Surgery. Anesthesiology. 2015;122(1):64-71. doi:10.1097/ ALN.0000000000000457

218. Südfeld S, Brechnitz S, Wagner JY, et al. Post-induction hypotension and early intraoperative hypotension associated with general anaesthesia. Br J Anaesth. 2017;119(1):57-64. doi:10.1093/bja/aex127

219. Stens J, Hering J-P, van der Hoeven CWP, et al. The added value of cardiac index and pulse pressure variation monitoring to mean arterial pressure-guided volume therapy in moderate-risk abdominal surgery (COGUIDE): a pragmatic multicentre randomised controlled trial. Anaesthesia. 2017;72(9):1078-1087. doi:10.1111/anae.13834

220. Yao Y-S, Qian B, Chen B-Z, Wang R, Tan L. The optimum concentration of levobupivacaine for intra-operative caudal analgesia in children undergoing inguinal hernia repair at equal volumes of injectate. Anaesthesia. 2009;64(1):23-26. doi:10.1111/j.1365-2044.2008.05688.x

221. Xu Z, Xu T, Zhao P, Ma R, Zhang M, Zheng J. Differential Roles of the Right and Left Toe Perfusion Index in Predicting the Incidence of Postspinal Hypotension During Cesarean Delivery. Anesth Analg. 2017;125(5):1560-1566. doi:10.1213/ANE.0000000000002393

222. Vetter TR, Carvallo D, Johnson JL, Mazurek MS, Presson RG. A comparison of single-dose caudal clonidine, morphine, or hydromorphone combined with ropivacaine in pediatric patients undergoing ureteral reimplantation. Anesth Analg. 2007;104(6):1356-63, table of contents. doi:10.1213/01.ane.0000261521.52562.de

223. Vernooij LM, van Klei WA, Machina M, Pasma W, Beattie WS, Peelen LM. Different methods of modelling intraoperative hypotension and their association with postoperative complications in patients undergoing non-cardiac surgery. Br J Anaesth. 2018;120(5):10801089. doi:10.1016/j.bja.2018.01.033

224. Jun I-G, Kwon H-M, Jung K-W, et al. The Impact of Postreperfusion Syndrome on Acute Kidney Injury in Living Donor Liver Transplantation. Anesth Analg. 2018;127(2):369-378. doi:10.1213/ ANE.0000000000003370

225. Dutta A, Sethi N, Sood J, et al. The Effect of Dexmedetomidine on Propofol Requirements During Anesthesia Administered by Bispectral Index-Guided Closed-Loop Anesthesia Delivery System. Anesth Analg. May 2018:1. doi:10.1213/ANE.0000000000003470 
226. Yoo KY, Jeong CW, Kang MW, et al. Bispectral index values during sevoflurane-nitrous oxide general anesthesia in women undergoing cesarean delivery: a comparison between women with and without prior labor. Anesth Analg. 2008;106(6):1827-1832. doi:10.1213/ ane.0b013e318172c4d7

227. Wink J, Veering BT, Aarts LPHJ, Wouters PF. Effect of increasing age on the haemodynamic response to thoracic epidural anaesthesia: an observational study. Eur J Anaesthesiol. 2014;31(11):597-605. doi:10.1097/EJA.0000000000000125

228. Richa F, Yazigi A, Sleilaty G, Yazbeck P. Comparison between dexmedetomidine and remifentanil for controlled hypotension during tympanoplasty. Eur J Anaesthesiol. 2008;25(5):369-374. doi:10.1017/S0265021508003761

229. van Zundert AAJ, Stultiens G, Jakimowicz JJ, et al. Laparoscopic cholecystectomy under segmental thoracic spinal anaesthesia: a feasibility study. Br J Anaesth. 2007;98(5):682-686. doi:10.1093/bja/aem058

230. Godet G, Lehot J-J, Janvier G, Steib A, De Castro V, Coriat P. Safety of HES 130/0.4 (Voluven(R)) in patients with preoperative renal dysfunction undergoing abdominal aortic surgery: a prospective, randomized, controlled, parallel-group multicentre trial. Eur J Anaesthesiol. 2008;25(12):986-994. doi:10.1017/S026502150800447X

231. Bilotta F, Caramia R, Paoloni FP, et al. Early postoperative cognitive recovery after remifentanil-propofol or sufentanil-propofol anaesthesia for supratentorial craniotomy: a randomized trial. Eur J Anaesthesiol. 2007;24(2):122-127. doi:10.1017/S0265021506001244

232. Yosry M, Othman IS. Controlled hypotension in adults undergoing choroidal melanoma resection: comparison between the efficacy of nitroprusside and magnesium sulphate. Eur J Anaesthesiol. 2008;25(11):891-896. doi:10.1017/S0265021508004584

233. Liao R, Li J, Liu J. Volatile induction/maintenance of anaesthesia with sevoflurane increases jugular venous oxygen saturation and lumbar cerebrospinal fluid pressure in patients undergoing craniotomy. Eur J Anaesthesiol. 2010;27(4):369-376. doi:10.1097/ EJA.0b013e32832edc70

234. Durmus M, But AK, Dogan Z, Yucel A, Miman MC, Ersoy MO. Effect of dexmedetomidine on bleeding during tympanoplasty or septorhinoplasty. Eur J Anaesthesiol. 2007;24(5):447-453. doi:10.1017/S0265021506002122

235. Pandey R, Garg R, Chandralekha, et al. Robot-assisted thoracoscopic thymectomy: perianaesthetic concerns. Eur J Anaesthesiol. 2010;27(5):473-477. doi:10.1097/ EJA.0b013e3283309cea

236. Song Y, Shim J-K, Song J-W, Kim E-K, Kwak Y-L. Dexmedetomidine added to an opioidbased analgesic regimen for the prevention of postoperative nausea and vomiting in highly susceptible patients: A prospective, randomised controlled trial. Eur J Anaesthesiol. August 2015. doi:10.1097/EJA.0000000000000327

237. Leslie K, Mcllroy D, Kasza J, et al. Neuraxial block and postoperative epidural analgesia: effects on outcomes in the POISE-2 trialt. Br J Anaesth. 2016;116(1):100-112. doi:10.1093/bja/ aev255 
Supplemental section 4. List of methods reported less than 5 times

Number of patients unresponsive to treatment

Incidence triple low

Number of patients with one hypotensive episode

Incidence exceeding exposure limit

Number of episodes per 100 hours

Number of liability episodes

Number of episodes followed by vasopressor

Number of alarms per hour

Average number of episodes

Number of exposure limit exceeded

Total duration triple low

Mean hypotension time per hour

Time below threshold per 100 hour

Time to minimum blood pressure

Time to onset hypotension

Time to resolve hypotension

Time between episodes

Double low per hour

Total duration double low

Proportional duration double low

Delay in onset hypotension

Time to detect hypotension

Time from onset to vasopressor

Time weighted average area under the curve (AUC)

Mean episode AUC

Maximum episode AUC

Relative fall below threshold

Mean blood pressure below threshold

Fractional mean arterial pressure

Blood pressure before vasopressor administration

Mean relative fall below baseline

Variance

Cumulative variation

Cumulative variation from baseline

Cumulative variation per time

Squared cumulative variation from baseline

Hölder exponents 
Width-at-half-height

Minimum performance error

Maximum performance error

Absolute number of measurements within threshold

Relative number of measurements outside threshold

Relative number of measurements doubles low 



\section{CHAPTER}

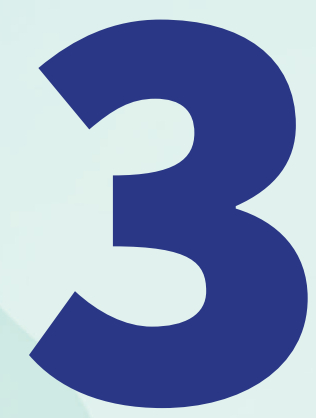

\section{Different methods of modelling}

intraoperative hypotension and their association with postoperative complications in patients undergoing non-cardiac surgery

Lisette M. Vernooij Wilton A. van Klei Karel G. M. Moons Judith A. van Waes Linda M. Peelen 


\section{ABSTRACT}

Background: Associations between intraoperative hypotension $(\mathrm{IOH})$ and postoperative complications have been reported. We examined whether using different methods to model $\mathrm{IOH}$ affected the association with postoperative myocardial injury (POMI) and acute kidney injury (AKI).

Methods: This two-centre cohort study included 10,432 patients aged $\geq 50$ years undergoing non-cardiac surgery. Twelve different methods to statistically model IOH (representing presence, depth, duration and area under the threshold (AUT)) were applied to examine the association with POMI and AKI using logistic regression analysis. To define $\mathrm{IOH}$, eight predefined thresholds were chosen.

Results: The incidences of POMI and AKI were $14.9 \%$ and $14.8 \%$ respectively. Different methods to model $\mathrm{IOH}$ yielded effect estimates differing in size and statistical significance. Methods with the highest odds were absolute maximum fall in blood pressure (BP) and mean episode AUT (odds ratio (OR) with 99\% confidence interval (Cl): 1.43 (1.15-1.77) and 1.69 (0.99-2.88), respectively, for the absolute mean arterial pressure $50 \mathrm{~mm} \mathrm{Hg}$ threshold). After standardization, the highest standardized odds ratios were obtained for depth related methods (OR with 99\% Cl: 1.12 (1.051.20) for absolute and relative maximum fall in BP). No single method always yielded the highest effect estimate in every setting. However, methods with the highest effect estimates remained consistent across different BP types, thresholds, outcomes and centres.

Conclusion: In studies on $\mathrm{IOH}$, both the threshold to define hypotension and the method chosen to model $\mathrm{IOH}$ affects the association of $\mathrm{IOH}$ with outcome. This makes different studies on $\mathrm{IOH}$ less comparable and hampers clinical application of reported results.

Key words: hypotension, models, statistical, troponin, acute kidney injury, intraoperative period 


\section{INTRODUCTION}

Intraoperative hypotension $(\mathrm{IOH})$ commonly occurs in patients undergoing non-cardiac surgery and has been a topic of growing research interest in recent years. (1-3) Serious postoperative complications have been associated with $\mathrm{IOH}$, including acute kidney injury (4-6), myocardial injury (5-7), stroke (8), delirium (9) and mortality. (10-12)

However, IOH has proven difficult to define. In a systematic review, 140 different definitions of $\mathrm{IOH}$ in terms of what threshold to use were found in 130 different scientific articles (13). Depending on the applied definition, the incidence of $1 \mathrm{OH}$ varied between 5\% and 99\%. Accordingly, the interpretation of reported associations based on $\mathrm{IOH}$ is complex.

Next to defining $\mathrm{IOH}$, no standardized methodology currently exists on how to express the severity of hypotension in statistical models. Commonly used methods to reflect clinical important $\mathrm{IOH}$ involve presence of hypotension, number of hypotensive episodes $(7,14)$, duration $(4,5,8)$, fall below blood pressure (BP) threshold (14) or a combination of the latter (expressed as area under the threshold). (7) Comparison of research papers reporting the same association between $\mathrm{IOH}$ and an outcome could be complicated if the method to reflect the severity of $\mathrm{IOH}$ varies between the publications. We hypothesize that different methods to model IOH will affect the association that is found between $\mathrm{IOH}$ and clinically important postoperative outcomes.

In this two-centre cohort study, we examined whether the method used to express the severity of $\mathrm{IOH}$ affects the association with postoperative outcomes. To check consistency of our findings, we conducted the comparison between methods to model $1 \mathrm{OH}$ using different thresholds to define $\mathrm{IOH}$, in two different hospitals, and on two postoperative outcomes, i.e. postoperative myocardial injury and postoperative acute kidney injury.

\section{METHODS}

\section{Study design and population}

This is a retrospective two-centre observational cohort study including patients undergoing non-cardiac surgery at the University Medical Center Utrecht (UMCU), Utrecht, the Netherlands between 1 January 2010 - 31 December 2012 or at the University Health Network Toronto (UHNT), Toronto, Canada between 2 January 2010 - 31 December 2014. In both hospitals, the local ethics committees approved the study protocol and waived the need for informed consent (UMC Utrecht Medical Research Ethics Committee, protocol number 12-425; University Health Network Research Ethics Board, protocol number 06-0193-AE, respectively). 
Eligible patients were aged $\geq 50$ years, underwent intermediate or high risk non-cardiac surgery under general or spinal anaesthesia with a postoperative hospital stay of $\geq 24$ hours. Re-operations within 30 days or within the same hospital admission were excluded from the analysis; if patients underwent surgery during another hospital admission at least 30 days after the first surgery, then this procedure was considered a novel case. Patients were excluded if intraoperative BP measurements were not available or if the anaesthesia duration was less than 20 minutes. In the UMCU, only patient's aged $\geq 60$ years were included as outcomes were not routinely measured in younger patients.

\section{Data collection}

All preoperative and postoperative data were collected previously from electronic medical and administrative records for another study from which the results have been reported elsewhere. (7) Intraoperative BP measurements were extracted from the anaesthesia information management system after designing the current study. Invasive BP measurements were recorded per minute, whereas non-invasive BP measurements were stored every 3-5 minutes. When both invasive and non-invasive BP measurements were available, the combination of both measurements was used to analyse $\mathrm{IOH}$.

\section{Thresholds for intraoperative hypotension}

As there is not a single threshold to define hypotension, we chose eight different thresholds; four absolute thresholds (i.e. mean arterial pressure (MAP) $<50 \mathrm{~mm} \mathrm{Hg}$, MAP $<60 \mathrm{~mm} \mathrm{Hg}$, systolic blood pressure $(\mathrm{SBP})<70 \mathrm{~mm} \mathrm{Hg}$, SBP $<90 \mathrm{~mm} \mathrm{Hg}$ ) and four relative thresholds (i.e. 20\% and $40 \%$ below baseline BP for both MAP and SBP). Relative thresholds were calculated with respect to baseline $\mathrm{BP}$, which was obtained for each patient by averaging all valid available BP measurements in the operating room before induction of anaesthesia. An algorithm to calculate time of induction has been previously described for the UMCU cohort. (13) In the UHNT cohort, time of induction was defined as the first end-tidal carbon dioxide reading. Patients were excluded from the analyses regarding relative thresholds if no baseline BP could be calculated due to unavailability of preinduction BP measurements.

\section{Methods to model intraoperative hypotension}

We selected twelve different methods to model $1 \mathrm{OH}$. Selection was based on a systematic literature search on methods that were often used to model IOH (Figure 1). These methods are either related to presence of $\mathrm{IOH}$, number of episodes, duration (total hypotensive duration, mean episode duration, maximal episode duration and time between different episodes), maximum depth below threshold (absolute maximum fall below threshold, relative maximum fall below threshold and mean BP under threshold) or area under the threshold (AUT) (total AUT, mean episode AUT per case, maximal episode AUT per case). 


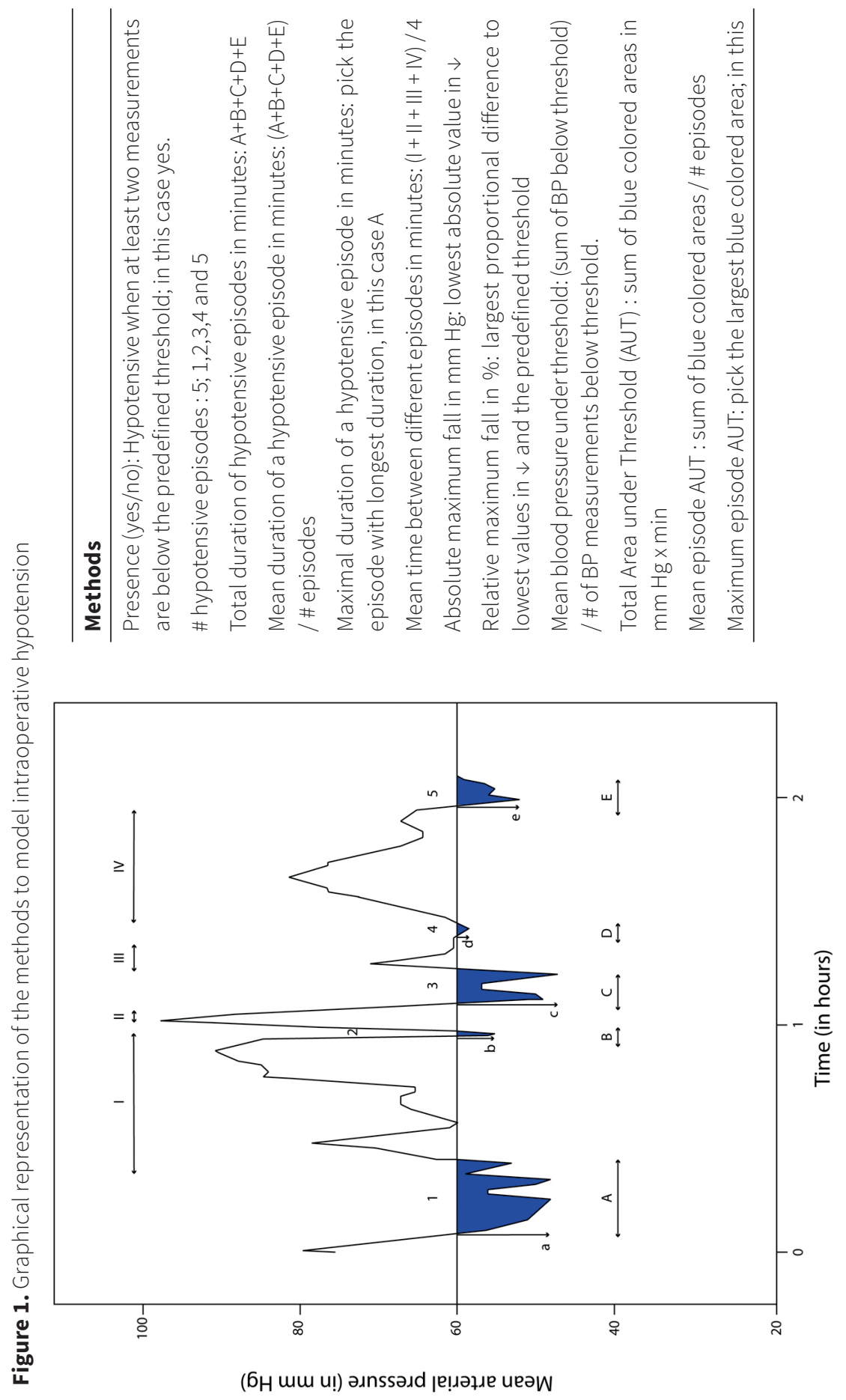


Methods regarding duration are reported per 10 minutes, methods regarding depth per $10 \mathrm{mmHg}$ and AUT in $100 \mathrm{mmHg}$ * minutes. An episode of $\mathrm{IOH}$ was defined as a continuing period of at least two single BP measurements below the threshold, as single BP measurements below the threshold were assumed to be possible artefacts. Consequently, single BP measurements above the predefined threshold during a hypotensive episode were not assumed the end of this episode.

\section{Postoperative myocardial injury}

The primary outcome was postoperative myocardial injury (POMI), defined as an elevation in cardiac troponin I above the $99^{\text {th }}$ percentile with a $10 \%$ variation. (2) Troponin I was routinely measured according to a local protocol in both hospitals. For the current analysis, the highest value of all routine troponin I measurements was used for each patient.

In the UMCU, troponin was measured routinely for the first three postoperative days and followup measurements were ordered according to the discretion of the attending cardiologist. (15) Troponin was analysed using a third-generation enhanced AccuTnl assay (Beckman Coulter, USA). Elevated troponin levels were based on a predefined clinical cut-off value of $>0.06 \mu \mathrm{g} / \mathrm{L}$.

In the UHNT, troponins were measured immediately after surgery and every 8 hours for the next two days. In case an elevation was observed during these two days, troponin was measured until postoperative day 5. Troponin I was determined using a Dade Behring Dimension assay (Siemens Healthcare Diagnostics, USA). Troponin elevation was determined using the predefined clinical cut-off value of $>0.07 \mu \mathrm{g} / \mathrm{L}$.

\section{Postoperative acute kidney injury}

Acute kidney injury (AKI) was included as a secondary outcome. Acute Kidney Injury Network (AKIN) criteria were used in the diagnosis of AKI (absolute increase of $0.3 \mathrm{mg} / \mathrm{dl}$ or relative increase of $50 \%$ of creatinine over the preoperative value during the first 48 hours after surgery). (16) As creatinine was not measured routinely in the UMCU, this part of the analysis was conducted in data from UHNT only. Preoperative creatinine measurements were performed routinely during the outpatient preoperative assessment.

\section{Potential confounders}

In our analyses we aimed to reflect that analyses on $\mathrm{IOH}$ are typically conducted in a multivariable setting, with adjustment for potential confounders. Therefore we adjusted for the following variables: age, sex, Revised Cardiac Risk Index (RCRI) factors (17) (i.e. high risk surgery, history of ischemic heart disease, history of cerebrovascular disease, chronic heart failure, elevated preoperative creatinine and insulin dependency), hypertension, peripheral vascular disease, use of chronic preoperative medication (i.e. beta blockers, calcium channel blocker, angiotensin- 
converting enzyme inhibitors and statins), ASA (American Society of Anesthesiologists) physical status, type of anaesthesia (i.e. general or spinal anaesthesia), surgical specialty (ear, nose, throat \& dental, general, gynaecological, neurological, orthopaedic, thoracic, urological and vascular) and centre (UMCU or UHNT). We defined heart failure as a left ventricular ejection fraction $<40 \%$ and ischemic heart disease as a previous myocardial infarction and/or coronary revascularization. Emergency surgery was defined as surgery required within 72 hours after the indication was set. Selection of these confounders was made a priori based on prior knowledge obtained from literature.

\section{Statistical analysis}

Baseline characteristics were compared between patients with or without POMI, patients who did or did not develop AKI and between patients being treated in either UMCU or UHNT, using the

chi-square test for categorical variables and Student t-test or Mann Whitney-U test for continuous variables as appropriate.

For every patient, $\mathrm{IOH}$ was expressed using each of the twelve methods described before. Univariable logistic regression analysis was performed to calculate the crude association between each of the methods and postoperative outcomes. Thereafter, multivariable logistic regression analysis was done with adjustment of aforementioned potential confounders. Effect estimates were expressed in odds ratios (OR) with their accompanying confidence intervals (CI). To take multiple testing into account, we tested against a $p$-value of 0.01 and used a $\mathrm{Cl}$ of 99\%. Bonferroni adjustment was deemed inappropriate and too conservative as the different methods to express $1 \mathrm{OH}$, and hence the tests, are highly dependent on each other. In addition, as the methods to model $\mathrm{IOH}$ are expressed in different units (e.g. time in minutes, depth in BP in $\mathrm{mm}$ $\mathrm{Hg}$ ), we repeated the analyses using standardized effect estimates to compare the association of each of the methods having a similar magnitude with postoperative outcomes. Furthermore, changes in Akaike's Information Criterion (AIC) and deviance were calculated to examine the incremental explained variance of the additive model compared to the baseline model. Change in AIC is defined as the difference between the baseline model (i.e. model consisting of all aforementioned confounders) and additive model (i.e. baseline model + one method to model $\mathrm{IOH})$. To assess the robustness of our findings we repeated the complete analytical approach described above for each of the eight predefined different thresholds to define $\mathrm{IOH}$. In addition, as we expected differences in anaesthetic management between centres, which may have influenced the association between $\mathrm{IOH}$ and postoperative outcomes, all analyses were repeated for each centre separately. Finally, we also repeated the analyses with AKI as the outcome of interest, using data from UHNT only. Altogether, comparisons between each of the methods were made over different thresholds to define $\mathrm{IOH}$, across different centres, blood pressure types (i.e. using either MAP or SBP), and outcomes. 
Missing values were mainly apparent in our data for postoperative troponin, preoperative creatinine, beta blocker prescription and hypertension. As missing data typically leads to bias effect estimates, we conducted multiple imputation using the mice library. (18-20) Imputation was performed per centre since the surgical population between both centres was not entirely comparable. All of the aforementioned analyses were conducted in all 50 imputation sets. Subsequently, results were pooled using Rubin's rule. For change in AIC the median change with the accompanying interquartile range over the imputation sets was reported. We did not conduct an a priori sample size analysis. All analyses were conducted using R software version 3.3.2 ( $R$ Core Team (2016) https://www.R-project.org).

\section{RESULTS}

There were 7,941 patients included in the UMCU and 2,491 patients in the UHNT (total 10,432 patients). Troponin was elevated in 1,560 (14.9\%) patients. Baseline BP was not available for 650 patients meaning that no analyses regarding relative BP thresholds could be performed, resulting in 9,782 included patients for these analyses (Supplementary Figure 1). The incidence of POMI in this population was $14.5 \%(n=1,416)$. Patient characteristics are described in Table 1.

\section{Incidence of $\mathrm{IOH}$}

Presence of at least one hypotensive episode occurred in 1,709 (16\%) of the patients using absolute MAP below $50 \mathrm{~mm} \mathrm{Hg}$ as a threshold and this incidence increased applying less stringent thresholds (51\%, 57\% and 93\% for absolute MAP $60 \mathrm{~mm} \mathrm{Hg}$, relative MAP 40\% and 20\% from baseline). Conversely, the incidence of POMI in hypotensive patients decreased using less stringent thresholds for $\mathrm{IOH}$ except for absolute MAP $50 \mathrm{~mm} \mathrm{Hg}$ (16\%, 19\%, 15\% and 14\%, respectively) (Supplementary table1). Similar patterns were observed for thresholds based on SBP. 
Table 1. Baseline characteristics in patients with and without postoperative myocardial injury

\begin{tabular}{|c|c|c|c|c|}
\hline & Overall & $\begin{array}{l}\text { No troponin } \\
\text { elevation }\end{array}$ & $\begin{array}{l}\text { Troponin } \\
\text { elevation }\end{array}$ & $\begin{array}{c}P \\
\text { value }\end{array}$ \\
\hline$n$ & 10432 & 8872 & 1560 & \\
\hline Sex (female) (\%) & $4505(43.2)$ & $3912(44.1)$ & $593(38.0)$ & $<0.001$ \\
\hline Age (mean (sd)) & $71.0(8.1)$ & $70.7(8.0)$ & $73.1(8.6)$ & $<0.001$ \\
\hline Peripheral vascular disease (\%) & $2160(20.7)$ & $1744(19.7)$ & $416(26.7)$ & $<0.001$ \\
\hline Hypertension (\%) & $5920(56.7)$ & $4920(55.5)$ & $1000(64.1)$ & $<0.001$ \\
\hline \multicolumn{5}{|l|}{ RCRI (\%) } \\
\hline High risk surgery & $4291(41.1)$ & $3426(38.6)$ & $865(55.4)$ & $<0.001$ \\
\hline History of ischemic heart disease & $1802(17.3)$ & $1338(15.1)$ & $464(29.7)$ & $<0.001$ \\
\hline History of chronic heart failure & $387(3.7)$ & $272(3.1)$ & $115(7.4)$ & $<0.001$ \\
\hline History of cerebrovascular disease & $1504(14.4)$ & $1271(14.3)$ & $233(14.9)$ & 0.553 \\
\hline Elevated creatinin & $591(5.7)$ & $349(3.9)$ & $242(15.5)$ & $<0.001$ \\
\hline Insulin dependent diabetes & $1284(12.3)$ & $1022(11.5)$ & $262(16.8)$ & $<0.001$ \\
\hline \multicolumn{5}{|l|}{ Chronic medication (\%) } \\
\hline$\beta$ blockers & $3223(30.9)$ & $2597(29.3)$ & $626(40.1)$ & $<0.001$ \\
\hline Calcium channel blockers & $2017(19.3)$ & $1655(18.7)$ & $362(23.2)$ & $<0.001$ \\
\hline Angiotensin renin blockers & $3866(37.1)$ & $3222(36.3)$ & $644(41.3)$ & $<0.001$ \\
\hline Statins & $4108(39.4)$ & $3379(38.1)$ & $729(46.7)$ & $<0.001$ \\
\hline ASA (\%) & & & & $<0.001$ \\
\hline 1 & $996(9.5)$ & $927(10.4)$ & $69(4.4)$ & \\
\hline 2 & $4907(47.0)$ & $4322(48.7)$ & $585(37.5)$ & \\
\hline 3 & $2935(28.1)$ & $2417(27.2)$ & $518(33.2)$ & \\
\hline 4 & $1594(15.3)$ & $1206(13.6)$ & $388(24.9)$ & \\
\hline Spinal anaesthesia (\%) & $699(6.7)$ & $634(7.1)$ & $65(4.2)$ & $<0.001$ \\
\hline Emergency surgery (\%) & $3000(28.8)$ & $2257(25.4)$ & $743(47.6)$ & $<0.001$ \\
\hline Duration of surgery (min) (median [IQR]) & $152[99,229]$ & $150[97,221]$ & $173[112,275]$ & $<0.001$ \\
\hline Surgical specialty (\%) & & & & $<0.001$ \\
\hline ENT \& dental & $1097(10.5)$ & $996(11.2)$ & $101(6.5)$ & \\
\hline General & $2176(20.9)$ & 1745 (19.7) & $431(27.6)$ & \\
\hline Gynaecological & $621(6.0)$ & $590(6.7)$ & $31(2.0)$ & \\
\hline Neurosurgery & $1628(15.6)$ & $1444(16.3)$ & $184(11.8)$ & \\
\hline Orthopaedic & $944(9.0)$ & $845(9.5)$ & $99(6.3)$ & \\
\hline Thoracic & $52(0.5)$ & $40(0.5)$ & $12(0.8)$ & \\
\hline Urological & $590(5.7)$ & $540(6.1)$ & $50(3.2)$ & \\
\hline Vascular & 3205 (30.7) & $2574(29.0)$ & $631(40.4)$ & \\
\hline Other/unknown & $119(1.1)$ & $98(1.1)$ & $21(1.3)$ & \\
\hline Center (UMCU) (\%) & $7941(76.1)$ & $6842(77.1)$ & $1099(70.4)$ & $<0.001$ \\
\hline
\end{tabular}

* UMCU: University Medical Center Utrecht; RCRI: Revised Cardiac Risk index; ASA: American Society of Anesthesiologists, ENT: ear, nose and throat. IQR: interquartile range. Values are number of patients (\%), unlesS indicated otherwise. 


\section{Method to model IOH exposure and POMI}

Depending on the thresholds to define $\mathrm{IOH}$, varying effect sizes were found using the different methods to express the severity of $\mathrm{IOH}$ (Table 2). Methods with the highest ORs were absolute maximum fall in BP, mean episode AUT and maximum episode AUT (ORs with accompanying 99\% Cl: 1.43 (1.15-1.77), 1.69 (0.99-2.88) and 1.21 (0.97-1.52), respectively for $1 \mathrm{OH}$ defined as absolute MAP $50 \mathrm{~mm} \mathrm{Hg}$ ). Less stringent thresholds resulted commonly in ORs closer to the value of 1 and the p-values were no longer significant (Table 2).

To allow comparison between methods with similar magnitudes, standardized ORs were calculated. Highest standardized ORs were observed for absolute and relative maximum fall in BP and mean BP under the threshold, which were 1.12 (99\% Cl; 1.05-1.20) for the methods related to fall in BP and 1.13 (1.05-1.21) for mean BP below the threshold. Differences in standardized ORs were observed between each of the methods to model IOH in association with POMI (Table 3). Compared to the unstandardized ORs, the standardized ORs were closer to each other with similar Cls levels of significance were similar between both (Table 2 and 3).

\section{Change in model performance measures}

For the absolute thresholds, depth related methods resulted overall in the highest deviance and thus, largest increment in explained variance (absolute and relative maximum fall in BP using deviance; 32.92, p<0.001 using the absolute MAP $50 \mathrm{~mm}$ Hg thresholds) (Table 3). Using relative thresholds, number of episodes showed consistently the largest increase in explained variance (deviance: 52.71, p $<0.001$ using the relative MAP 40\% from baseline as a threshold) (Supplementary table 5). Similar results were found using change in AIC as a measure for model performance (Table 3 and 4 and Supplementary table 5-8, 13-16). 


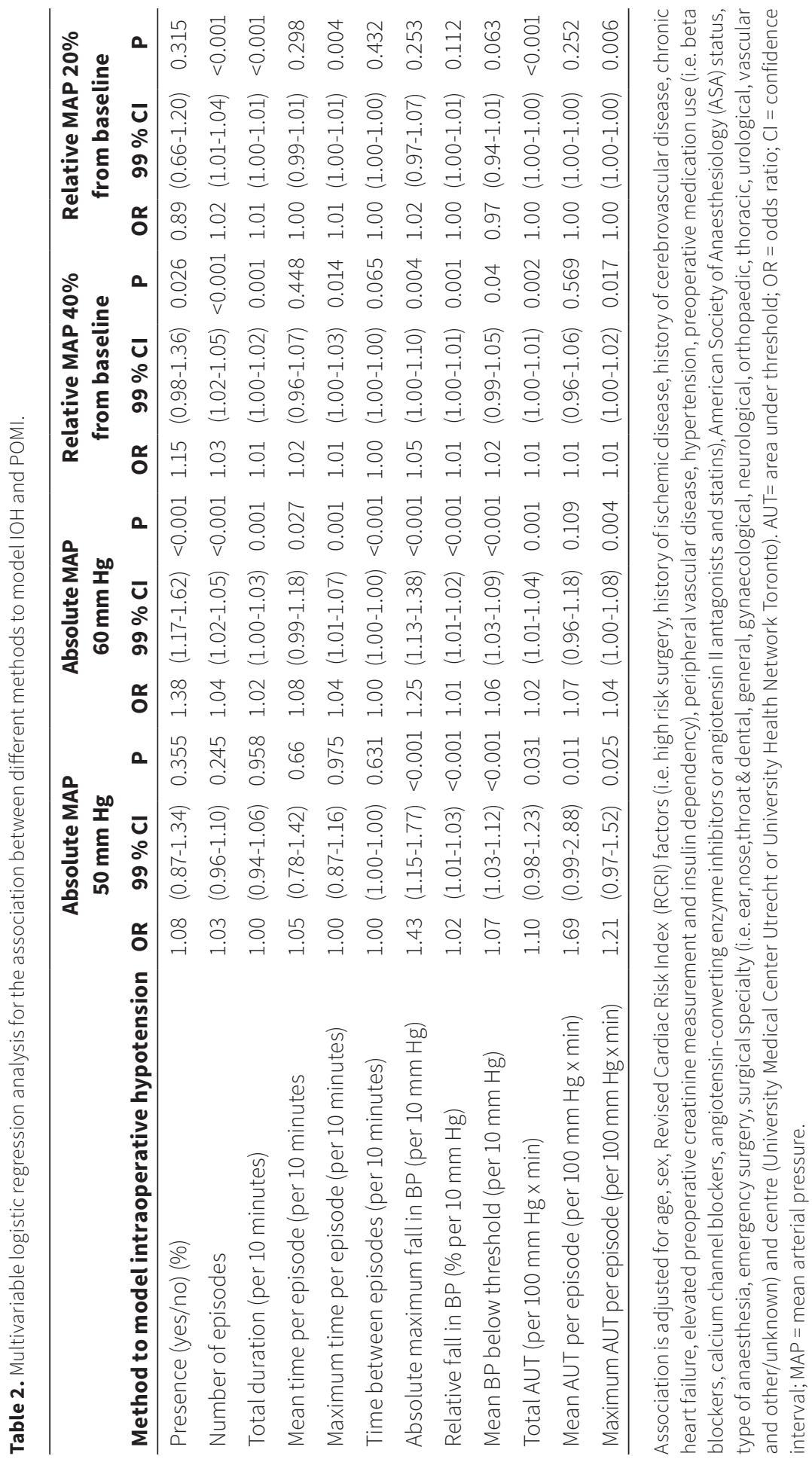




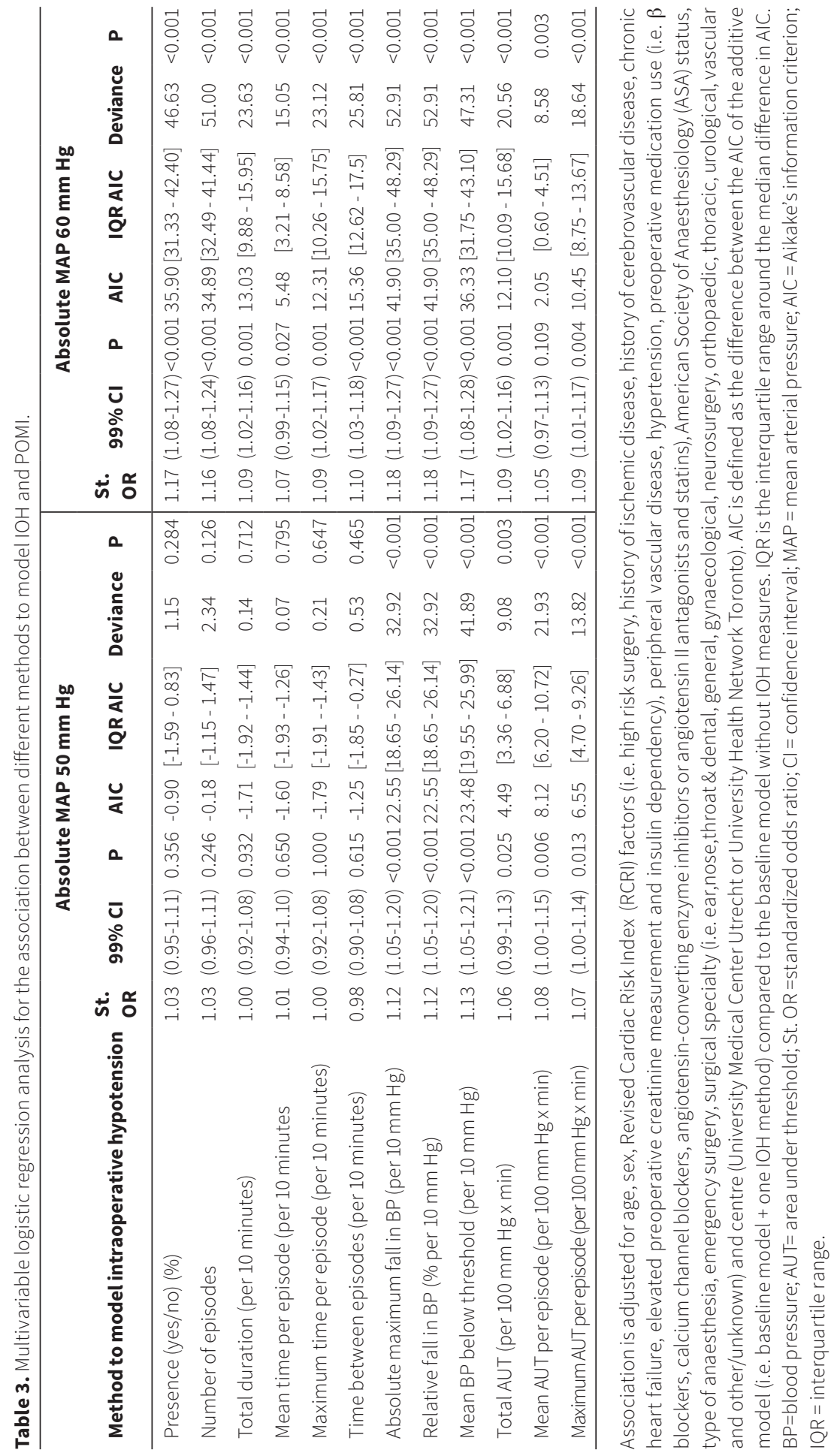




\section{Acute kidney injury}

AKI was diagnosed in 370 (14.8\%) patients of the total 2,491 patients included in the UHNT cohort. Similar results in patterns of methods with increasing ORs, as well as in the standardized estimates analyses, were observed using AKI as an outcome compared to POMI (Table 4). Methods with the largest standardized ORs were: presence of $\mathrm{IOH}$, mean episode duration, absolute fall in BP and mean episode AUT (standardized OR with accompanying 99\% CI: 1.17 (1.00-1.36) using absolute

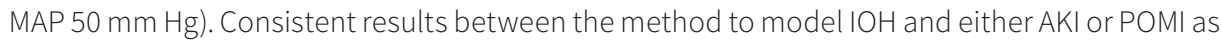
an outcome were found (Supplementary table 11-15).

\section{Patterns in effect estimates across centre and blood pressure type}

No single method to express severity of $\mathrm{IOH}$ was found to be consistently more significantly associated or had a higher effect estimate compared to other methods. However, consistent patterns were found for the association between each of the method to model IOH and POMI for both individual centres (Figure 2). This consistency was observed in both the standardized and the unstandardized associations and the model performance measures. Furthermore, using SBP as the BP type to define $\mathrm{IOH}$, similar patterns in effect sizes for different methods to express $\mathrm{IOH}$ were also observed (Supplementary table 5 and 6).

Figure 2. Graphical representation of the standardized associations between method to model $1 \mathrm{OH}$ with POMI per centre for the absolute threshold MAP $<50 \mathrm{~mm} \mathrm{Hg}$

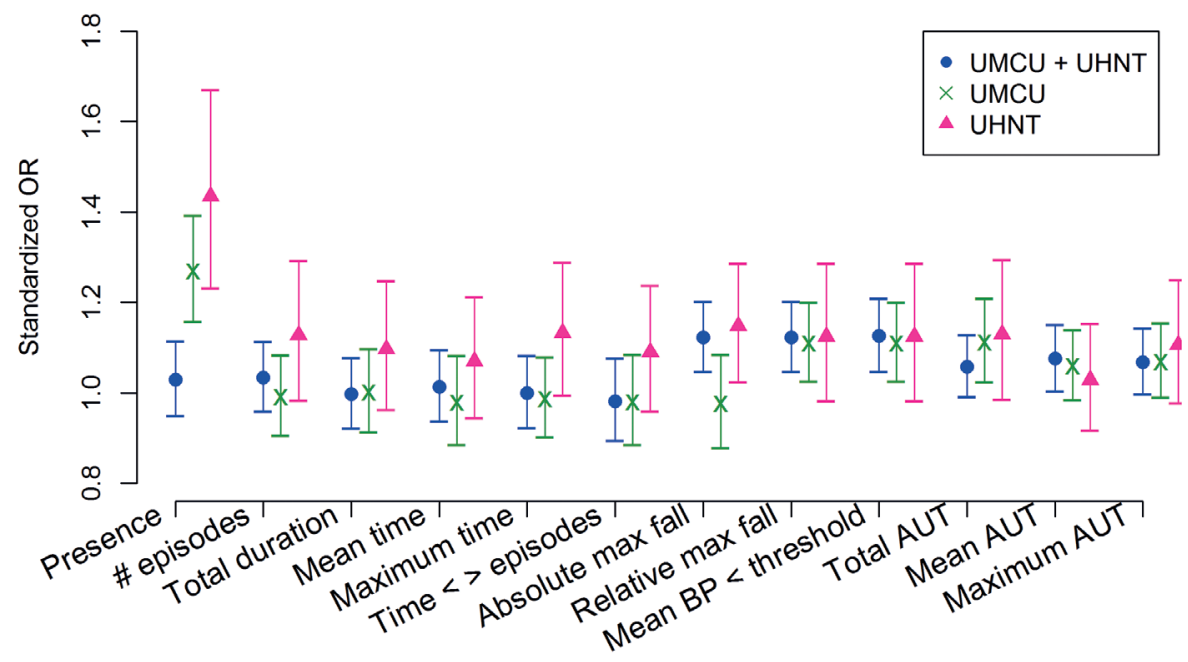




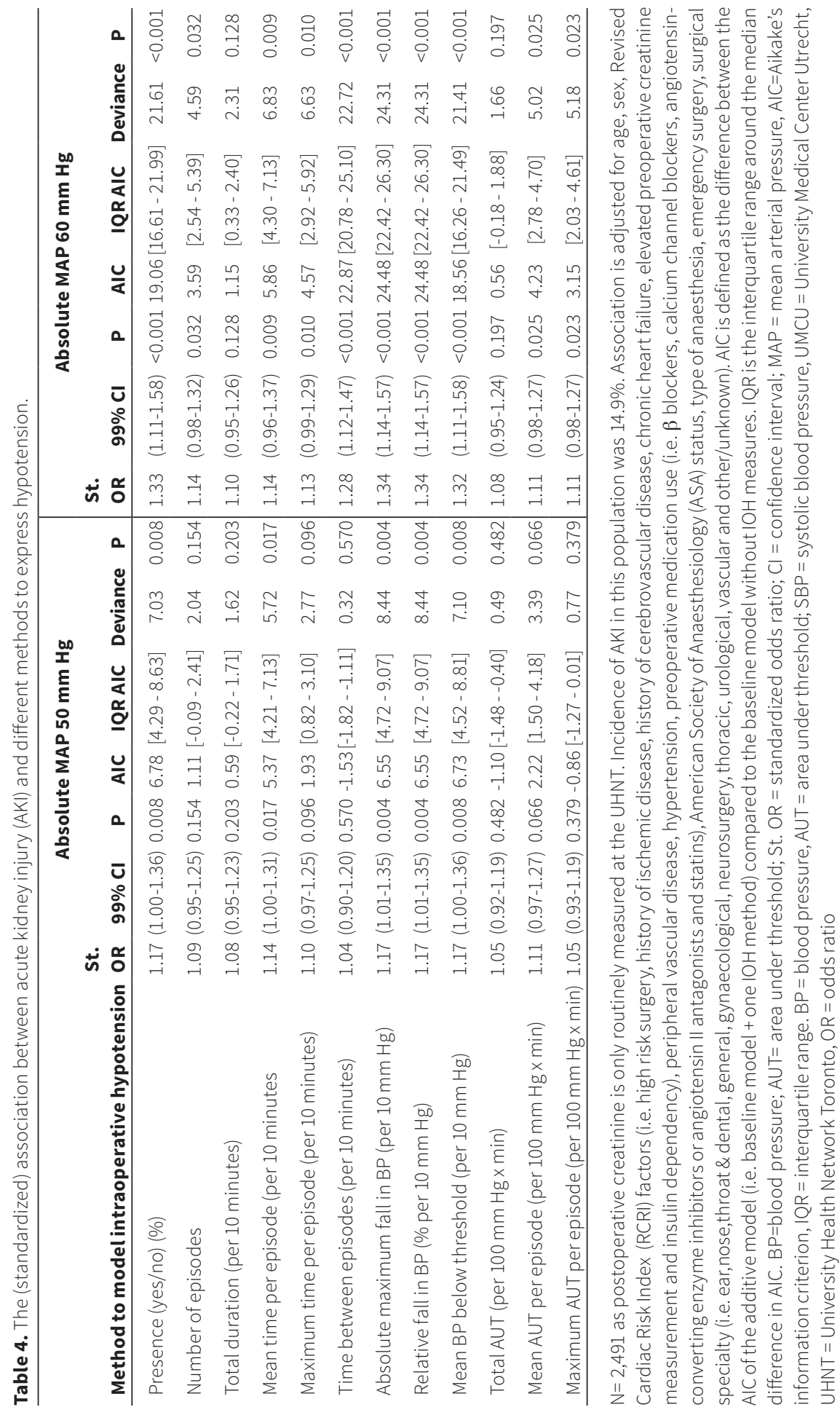




\section{DISCUSSION}

In this observational study, as expected higher incidences of $\mathrm{IOH}$ were observed with less stringent thresholds to define $\mathrm{IOH}$, whereas the incidence of POMI and AKI in hypotensive patients decreased with less stringent thresholds. Most important, using different methods varying effects estimates were observed (standardized OR 0.98 to 1.12; using absolute MAP $50 \mathrm{~mm}$ Hg as a threshold) with highly different levels of significance $(<0.001$ to 0.93 ). This indicates that the method to model IOH strongly influences the magnitude of the association that is found. Model performance measures, such as change in AIC and deviance, showed consistently largest increased explained variance for depth-related measures using absolute thresholds, and for number of episodes using relative thresholds. Furthermore, consistency in our results was found for two blood pressure types (MAP and SBP), for two postoperative outcomes (i.e. POMI and AKI) and between both centres. However, there was not a single method which in all settings yielded the strongest effect estimate. Therefore, it is yet too early to make definite recommendations on what method best to use in studying $\mathrm{IOH}$ and its effects on adverse outcomes.

Currently, no clear cut-off points in the definitions of hypotension are described in literature. Bijker et al. found 140 different definitions of thresholds defining hypotension in 130 articles, which resulted in increasing incidences of $\mathrm{IOH}$ with less stringent thresholds. (13) Our results support these findings as we found similar patterns of incidence of hypotension over the different thresholds applied.

Besides the lack of clarity for the threshold definition of $\mathrm{IOH}$, no consensus exists on what method to use in modelling $\mathrm{IOH}$ as potential determinant of an outcome. Investigators use different methods or different combinations of methods to test the association of $\mathrm{IOH}$ with postoperative outcomes. For example, different researchers studied the association between $\mathrm{IOH}$ and mortality. (10-12) Bijker et al. used maximum episode duration, (12) Mascha et al. used blood pressure variability (11) and Monk et al. used total hypotensive duration, AUT and mean BP under thresholds (10) as measures for $\mathrm{IOH}$. In addition, Walsh et al. and Salmasi et al. investigated both POMI and AKI, van Waes et al. investigated POMI and Sun et al. studied AKI in relation to IOH. (4-7) Again, different methods were used studying the same association. Consequently, comparison of the results between different studies should be interpreted with caution.

This study has several strengths. To our knowledge, this is the first study in which different methods to model $\mathrm{IOH}$ in relation to postoperative outcomes are compared with each other. Second, we included patients from two different centres where troponin is routinely measured resulting in increased generalizability compared to inclusion of patients treated in a single centre. Furthermore, we tested the association using two different outcomes, i.e. POMI and AKI. Finally, 
patterns in results were consistent over different thresholds, different blood pressure types (i.e. MAP and SBP), different centres and different outcomes which implicate high generalizability.

However, several aspects should be taken into account. Firstly, in our analyses we only focused on $1 \mathrm{OH}$ adjusted for preoperative factors. However, several intraoperative factors were found to be associated with adverse postoperative outcomes as well, such as estimated blood loss, $(4,5,9)$ number of blood transfusions $(21,22)$ and surgical duration. $(4,9,11,12,21,22)$ We did not include these intraoperative factors in our analyses as they are closely correlated with hypotension exposure. In addition, as we focus on the comparison of methods we do not expect this to result in different conclusions of our study.

Secondly, pre-induction BP measured within the operating room was used as baseline BP in the analyses with relative thresholds. However, on average pre-induction BP is higher compared to blood pressure measured in the pre-operative clinic. $(23,24)$ This implicates that the results using relative thresholds could on average be estimated too high leading to a potential underestimation of the found associations. Thirdly, only patients from the UHNT were included for the analyses between methods to model $\mathrm{IOH}$ and AKI as creatinine is not routinely measured at the UMCU. Due to the smaller sample size, wider Cls and less significant results were found as compared to the results based on POMI. Lastly, multiple testing of the BP measurements occurred in defining hypotension and comparing the different methods. Fourthly, dependence in the data could have been introduced by including patients during another hospital admission at least 30 days after the first surgery. Exposure to surgery and $\mathrm{IOH}$ could increase the probability of events in these patients. As we have adjusted for pre-operative patient characteristics and due to the presumably low number of patients, we believe this would not have affected the interpretation of our results and conclusion. Finally, as the analyses and BP measurements were highly dependent on each other, we decided to test against a p-value of 0.01 as adjustment using the Bonferroni correction is too conservative. However, as our primary aim was to compare different methods to model $\mathrm{IOH}$, we expect no differences in the interpretation of our results.

In summary, our study shows differences in effect estimates with respect to the method used in modelling $\mathrm{IOH}$ in association with postoperative outcomes. However, consistent patterns of these effect estimates were observed across different thresholds, centres, BP types and outcomes. Researchers should be aware that differences in defining and modelling $\mathrm{IOH}$ are present and that reporting of how to define $\mathrm{IOH}$ and what method is used to express the severity of $\mathrm{IOH}$ is mandatory when studying an association between $\mathrm{IOH}$ and postoperative outcome. As $\mathrm{IOH}$ has been reported to be associated with serious adverse clinical outcomes, there is a need for consensus on which method to use in modelling $\mathrm{IOH}$ and the threshold to define hypotension to improve comparability between different studies reporting similar associations. 


\section{REFERENCES}

1. Landesberg G, Beattie WS, Mosseri M, Jaffe AS, Alpert JS. Perioperative myocardial infarction. Circulation [Internet]. 2009 Jul 9 [cited 2015 Aug 31];119(22):2936-44. Available from: http:// www.ncbi.n/m.nih.gov/pubmed/19506125

2. Thygesen K, Alpert JS, Jaffe AS, Simoons ML, Chaitman BR, White HD, et al. Third universal definition of myocardial infarction. Circulation [Internet]. 2012 Oct 16 [cited 2015 Jun 1];126(16):2020-35. Available from: http://www.ncbi.nlm.nih.gov/pubmed/22923432

3. Devereaux PJ, Sessler DI. Cardiac Complications in Patients Undergoing Major Noncardiac Surgery. N Engl J Med [Internet]. 2015 Dec 3 [cited 2015 Dec 3];373(23):2258-69. Available from: http://www.ncbi.nlm.nih.gov/pubmed/26630144

4. Sun LY, Wijeysundera DN, Tait GA, Beattie WS. Association of Intraoperative Hypotension with Acute Kidney Injury after Elective Noncardiac Surgery. Anesthesiology [Internet]. 2015 Sep 1 [cited 2015 Nov 2];123(3):515-23. Available from: http://anesthesiology.pubs.asahq. org/article.aspx?articleid=2411181

5. Walsh M, Devereaux PJ, Garg AX, Kurz A, Turan A, Rodseth RN, et al. Relationship between intraoperative mean arterial pressure and clinical outcomes after noncardiac surgery: toward an empirical definition of hypotension. Anesthesiology [Internet]. 2013 Sep [cited 2015 Feb 23];119(3):507-15. Available from: http://www.ncbi.nlm.nih.gov/pubmed/23835589

6. Salmasi V, Maheshwari K, Yang D, Mascha EJ, Singh A, Sessler DI, et al. Relationship between Intraoperative Hypotension, Defined by Either Reduction from Baseline or Absolute Thresholds, and Acute Kidney and Myocardial Injury after Noncardiac Surgery: A Retrospective Cohort Analysis. Anesthesiology [Internet]. 2017 Jan [cited 2017 Jul 31];126(1):47-65. Available from: http://insights.ovid.com/crossref?an=00000542-201701000-00017

7. van Waes JAR, van Klei WA, Wijeysundera DN, van Wolfswinkel L, Lindsay TF, Beattie WS. Association between Intraoperative Hypotension and Myocardial Injury after Vascular Surgery. Anesthesiology [Internet]. 2016 Jan [cited 2015 Dec 22];124(1):35-44. Available from: http://www.ncbi.nlm.nih.gov/pubmed/26540148

8. Bijker JB, Persoon S, Peelen LM, Moons KGM, Kalkman CJ, Kappelle LJ, et al. Intraoperative hypotension and perioperative ischemic stroke after general surgery: a nested case-control study. Anesthesiology [Internet]. 2012 Mar [cited 2015 Apr 23];116(3):658-64. Available from: http://www.ncbi.nlm.nih.gov/pubmed/22277949

9. Hirsch J, DePalma G, Tsai TT, Sands LP, Leung JM. Impact of intraoperative hypotension and blood pressure fluctuations on early postoperative delirium after non-cardiac surgery†. Br J Anaesth [Internet]. 2015 Jan 23 [cited 2015 Feb 2]; Available from: http://www.ncbi.nlm.nih. gov/pubmed/25616677

10. Monk TG, Bronsert MR, Henderson WG, Mangione MP, Sum-Ping STJT, Bentt DR, et al. Association between Intraoperative Hypotension and Hypertension and 30-day Postoperative Mortality in Noncardiac Surgery. Anesthesiology [Internet]. 2015 Jun 17 [cited 2015 Jun 23]; Available from: http://www.ncbi.nlm.nih.gov/pubmed/26083768

11. Mascha EJ, Yang D, Weiss S, Sessler DI. Intraoperative Mean Arterial Pressure Variability and 30-day Mortality in Patients Having Noncardiac Surgery. Anesthesiology [Internet]. $2015 \mathrm{Apr}$ 30 [cited 2015 Jul 10]; Available from: http://www.ncbi.nlm.nih.gov/pubmed/25929547 
12. Bijker JB, van Klei WA, Vergouwe Y, Eleveld DJ, van Wolfswinkel L, Moons KGM, et al. Intraoperative hypotension and 1-year mortality after noncardiac surgery. Anesthesiology [Internet]. 2009 Dec [cited 2015 Apr 23];111(6):1217-26. Available from: http://www.ncbi.nlm. nih.gov/pubmed/19934864

13. Bijker JB, van Klei WA, Kappen TH, van Wolfswinkel L, Moons KGM, Kalkman CJ. Incidence of Intraoperative Hypotension as a Function of the Chosen Definition. Anesthesiology [Internet]. 2007 Aug [cited 2015 Mar 15];107(2):213-20. Available from: http://www.ncbi.nlm. nih.gov/pubmed/17667564

14. Chen G, Chung E, Meng L, Alexander B, Vu T, Rinehart J, et al. Impact of non invasive and beat-to-beat arterial pressure monitoring on intraoperative hemodynamic management. J Clin Monit Comput [Internet]. 2012 Apr [cited 2015 May 12];26(2):133-40. Available from: http://www.ncbi.nlm.nih.gov/pubmed/22382920

15. van Waes JAR, Nathoe HM, de Graaff JC, Kemperman H, de Borst GJ, Peelen LM, et al. Myocardial injury after noncardiac surgery and its association with short-term mortality. Circulation [Internet]. 2013 Jun 11 [cited 2015 May 27];127(23):2264-71. Available from: http:// www.ncbi.nlm.nih.gov/pubmed/23667270

16. Mehta RL, Kellum JA, Shah S V, Molitoris BA, Ronco C, Warnock DG, et al. Acute Kidney Injury Network: report of an initiative to improve outcomes in acute kidney injury. Crit Care [Internet]. 2007 Jan [cited 2014 Jul 14];11(2):R31. Available from: http://www.pubmedcentral. nih.gov/articlerender.fcgi?artid=2206446\&tool=pmcentrez\&rendertype=abstract

17. Lee TH, Marcantonio ER, Mangione CM, Thomas EJ, Polanczyk CA, Cook EF, et al. Derivation and prospective validation of a simple index for prediction of cardiac risk of major noncardiac surgery. Circulation [Internet]. 1999 Sep 7 [cited 2016 May 1];100(10):1043-9. Available from: http://www.ncbi.nlm.nih.gov/pubmed/10477528

18. Donders ART, van der Heijden GJMG, Stijnen T, Moons KGM. Review: a gentle introduction to imputation of missing values. J Clin Epidemiol [Internet]. 2006 Oct [cited 2016 Dec 8];59(10):1087-91. Available from: http://linkinghub.elsevier.com/retrieve/pii/ S0895435606001971

19. Little RJA. Regression with Missing X's: A Review. J Am Stat Assoc [Internet]. 1992 Dec [cited 2016 Dec 8];87(420):1227-37. Available from: http://www.tandfonline.com/doi/abs/10.1080/ 01621459.1992 .10476282

20. Buuren S van, Groothuis-Oudshoorn K. mice : Multivariate Imputation by Chained Equations in R. J Stat Softw [Internet]. 2011 [cited 2016 Dec 8];45(3):1-67. Available from: http://www. jstatsoft.org/v45/i03/

21. Kheterpal S, O'Reilly M, Englesbe MJ, Rosenberg AL, Shanks AM, Zhang L, et al. Preoperative and intraoperative predictors of cardiac adverse events after general, vascular, and urological surgery. Anesthesiology [Internet]. 2009 Jan [cited 2015 Apr 23];110(1):58-66. Available from: http://www.ncbi.nlm.nih.gov/pubmed/19104171

22. Sabaté S, Mases A, Guilera N, Canet J, Castillo J, Orrego C, et al. Incidence and predictors of major perioperative adverse cardiac and cerebrovascular events in non-cardiac surgery. $\mathrm{Br}$ J Anaesth [Internet]. 2011 Dec [cited 2015 Mar 21];107(6):879-90. Available from: http://www. ncbi.nlm.nih.gov/pubmed/21890661 
23. van Klei WA, van Waes JAR, Pasma W, Kappen TH, van Wolfswinkel L, Peelen LM, et al. Relationship Between Preoperative Evaluation Blood Pressure and Preinduction Blood Pressure. Anesth Analg [Internet]. 2017 Feb [cited 2017 Dec 21];124(2):431-7. Available from: http://www.ncbi.nlm.nih.gov/pubmed/27755054

24. Drummond JC, Blake JL, Patel PM, Clopton P, Schulteis G. An observational study of the influence of "white-coat hypertension" on day-of-surgery blood pressure determinations. J Neurosurg Anesthesiol [Internet]. 2013 Apr [cited 2015 Oct 30];25(2):154-61. Available from: http://www.ncbi.nlm.nih.gov/pubmed/23211641 


\section{SUPPLEMENTAL MATERIAL}

\section{Supplemental Figure 1. Patient flow chart}

Patients $\geq 50$ years*, underwent intermediate or

high risk non-cardiac surgery, postoperative stay $\geq$

24 hours $n=10,432$

\begin{tabular}{l|l} 
UMCU $n=7,941$ & UHNT $n=2,491$
\end{tabular}

\begin{tabular}{|c|r|}
\hline \multicolumn{2}{|l|}{ Patients with baseline BP } \\
\hline UMCU $n=7,356$ & UHNT $n=2,426$ \\
\hline
\end{tabular}

*In the UMCU patients aged $\geq 60$ years were included as troponin is only measured in these patients. UMCU = University Medical Center Utrecht; UHNT = University Health Network Toronto 
Supplemental Table. Incidence of POMI and hypotension for different thresholds to define hypotension

\begin{tabular}{lcc}
\hline & Hypotension & $\begin{array}{c}\text { Hypotensive patients with } \\
\text { troponin elevation }\end{array}$ \\
\hline Absolute MAP $50 \mathrm{~mm} \mathrm{Hg}(\%)$ & $1709(16.4)$ & $277(16.2)$ \\
Absolute MAP 60 mm Hg (\%) & $5295(50.8)$ & $991(18.7)$ \\
Relative MAP 40\% from baseline (\%) & $5565(56.6)$ & $820(14.7)$ \\
Relative MAP 20\% from baseline (\%) & $9118(92.8)$ & $1287(14.1)$ \\
Absolute SBP 70 mm Hg (\%) & $1215(11.6)$ & $290(23.9)$ \\
Absolute SBP 90 mm Hg (\%) & $6952(66.6)$ & $1126(16.2)$ \\
Relative SBP 40\% from baseline (\%) & $6087(61.9)$ & $875(14.4)$ \\
Relative SBP 20\% from baseline (\%) & $9241(94.0)$ & $1313(14.2)$ \\
\hline
\end{tabular}

* $n=10,432$ for absolute thresholds, $n=9,828$ for mean arterial pressure (MAP) thresholds, $n=9,834$ for relative systolic blood pressure (SBP) thresholds.

Supplemental Table 2. Baseline characteristics of patients without missing values and patients with at least 1 missing value

\begin{tabular}{|c|c|c|c|}
\hline & Complete case & At least 1 missing & P value \\
\hline$n$ & 3627 & 6805 & \\
\hline Sex (female) (\%) & $2092(58)$ & $3825(56)$ & 0.201 \\
\hline Age (mean (sd)) & $71.2(7.8)$ & $71.0(8.3)$ & 0.198 \\
\hline Peripheral vascular disease (\%) & $698(19.2)$ & $1462(21.5)$ & 0.008 \\
\hline Hypertension (\%) & $2123(58.5)$ & 3009 (52.5) & $<0.001$ \\
\hline \multicolumn{4}{|l|}{ RCRI (\%) } \\
\hline High risk surgery & $1467(40.4)$ & $2824(41.5)$ & 0.308 \\
\hline History of ischemic heart disease & $553(15.2)$ & $1243(18.4)$ & $<0.001$ \\
\hline History of chronic heart failure & $145(4.0)$ & $242(3.6)$ & 0.279 \\
\hline History of cerebrovascular disease & $564(15.6)$ & $940(13.8)$ & 0.018 \\
\hline Elevated creatinin & $208(5.7)$ & $379(7.3)$ & 0.005 \\
\hline Insulin dependent diabetes & $354(9.8)$ & $930(13.7)$ & $<0.001$ \\
\hline \multicolumn{4}{|l|}{ Chronic medication (\%) } \\
\hline$\beta$ blockers & $1205(33.2)$ & $1714(30.1)$ & 0.002 \\
\hline Calcium channel blockers & $717(19.8)$ & $1300(19.2)$ & 0.51 \\
\hline Angiotensin renin blockers & $1458(40.2)$ & $2406(35.6)$ & $<0.001$ \\
\hline Statins & $1477(40.7)$ & $2631(38.9)$ & 0.069 \\
\hline
\end{tabular}


Supplemental Table 2. Baseline characteristics of patients without missing values and patients with at least 1 missing value

\begin{tabular}{lccc}
\hline & Complete case & At least 1 missing & P value \\
\hline ASA (\%) & & & $<0.001$ \\
1 & $289(8.0)$ & $707(10.4)$ & \\
2 & $1880(51.8)$ & $3027(44.5)$ & \\
3 & $1121(30.9)$ & $1814(26.7)$ & \\
4 & $337(9.3)$ & $1257(18.5)$ & \\
Spinal anaesthesia (\%) & $175(4.8)$ & $524(7.7)$ & $<0.001$ \\
Emergency surgery (\%) & $795(21.9)$ & $2205(32.4)$ & $<0.001$ \\
Surgical specialty (\%) & & & $<0.001$ \\
ENT \& dental & $370(10.2)$ & $727(10.7)$ & \\
General & $834(23.0)$ & $1342(19.7)$ & \\
Gynaecological & $200(5.5)$ & $421(6.2)$ & \\
Neurosurgery & $684(18.9)$ & $944(13.9)$ & \\
Orthopaedic & $338(9.3)$ & $606(8.9)$ & \\
Thoracic & $27(0.7)$ & $25(0.4)$ & \\
Urological & $216(6.0)$ & $374(5.5)$ & \\
Vascular & $907(25.0)$ & $2298(33.8)$ & \\
Other/unknown & $51(1.4)$ & $68(1.0)$ & \\
POMl (\%) & $808(22.3)$ & $625(21.1)$ & 0.277 \\
Centre (UMC Utrecht) (\%) & $3065(84.5)$ & $4876(71.7)$ & $<0.001$ \\
\hline
\end{tabular}

RCRI = Revised Cardiac Risk Index; ASA = American Society of Anesthesiologists; ENT = ear, nose throat; UMC Utrecht = University Medical Center Utrecht; POMI = postoperative myocardial injury. Values are numbers of patients (\%), unless indicated otherwise. Missing values were present for hypertension ( $n=984)$, elevated creatinine $(n=843)$, beta blockers $(n=983)$, ischemic heart disease $(n=29)$, calcium blockers and angiotensin renin blockers ( $n=28)$, statins $(n=27)$ and ASA $(n=1)$. Surgical specialty (\%) ENT \& dental General Gynaecological Neurosurgery Orthopaedic Thoracic Urological Vascular Other/unknown POMI (\%) 
Supplemental Digital Content, Table 3. Baseline characteristics of patients treated in University Medical Center Utrecht or University Health Network Toronto.

\begin{tabular}{|c|c|c|c|}
\hline & UHNT & UMCU & P value \\
\hline$n$ & 2491 & 7941 & \\
\hline Sex (female) (\%) & $766(31.0)$ & $3739(47.0)$ & $<0.001$ \\
\hline Age (mean (sd)) & $70.1(9.7)$ & $71.2(7.6)$ & 0.002 \\
\hline Peripheral vascular disease (\%) & $1155(46.4)$ & $1005(12.7)$ & $<0.001$ \\
\hline Hypertension (\%) & $1887(75.8)$ & $4033(50.8)$ & $<0.001$ \\
\hline \multicolumn{4}{|l|}{ RCRI (\%) } \\
\hline High risk surgery & $2042(82.0)$ & $2249(28.3)$ & $<0.001$ \\
\hline History of ischemic heart disease & $954(38.3)$ & $848(10.7)$ & $<0.001$ \\
\hline History of chronic heart failure & $159(6.4)$ & $228(2.9)$ & $<0.001$ \\
\hline History of cerebrovascular disease & $267(10.7)$ & $1237(15.6)$ & $<0.001$ \\
\hline Elevated creatinin & $174(7.0)$ & $417(5.3)$ & 0.001 \\
\hline Insulin dependent diabetes & $769(30.9)$ & $515(6.5)$ & $<0.001$ \\
\hline \multicolumn{4}{|l|}{ Chronic medication (\%) } \\
\hline$\beta$ blockers & $817(32.8)$ & $2406(30.3)$ & 0.020 \\
\hline Calcium channel blockers & $716(28.7)$ & $1301(16.4)$ & $<0.001$ \\
\hline Angiotensin renin blockers & $1048(42.1)$ & $2818(35.5)$ & $<0.001$ \\
\hline Statins & $1370(55.0)$ & $2738(34.5)$ & $<0.001$ \\
\hline ASA (\%) & & & $<0.001$ \\
\hline 1 & $6(0.2)$ & $990(12.5)$ & \\
\hline 2 & $67(2.7)$ & $4840(60.9)$ & \\
\hline 3 & $923(37.1)$ & $2012(25.3)$ & \\
\hline 4 & $1495(60.0)$ & $99(1.2)$ & \\
\hline Spinal anaesthesia (\%) & $159(6.4)$ & $540(6.8)$ & 0.496 \\
\hline Emergency surgery (\%) & $661(26.5)$ & $2339(29.5)$ & 0.005 \\
\hline Duration of surgery in min (median [IQR]) & $198[138,277]$ & $140[93,210]$ & $<0.001$ \\
\hline
\end{tabular}


Supplemental Digital Content, Table 3. Baseline characteristics of patients treated in University Medical Center Utrecht or University Health Network Toronto.

\begin{tabular}{lccc}
\hline & UHNT & UMCU & P value \\
\hline Surgical specialty (\%) & & & $<0.001$ \\
ENT \& dental & $92(3.7)$ & $1005(12.7)$ & \\
General & $112(4.5)$ & $2064(26.0)$ & \\
Gynaecological & $59(2.4)$ & $562(7.1)$ & \\
Neurosurgery & $0(0.0)$ & $1628(20.5)$ & \\
Orthopaedic & $0(0.0)$ & $944(11.9)$ & \\
Thoracic & $52(2.1)$ & $0(0.0)$ & \\
Urological & $177(7.1)$ & $413(5.2)$ & \\
Vascular & $1990(79.9)$ & $1215(15.3)$ & \\
Other/unknown & $9(0.4)$ & $110(1.4)$ & \\
POMI (\%) & $461(18.5)$ & $1099(13.8)$ & $<0.001$ \\
\hline
\end{tabular}

RCRI = Revised Cardiac Risk Index; ASA = American Society of Anesthesiologists; ENT = ear, nose throat; UHNT = University Health Network Toronto, UMCU = University Medical Center Utrecht; POMI = postoperative myocardial injury. Values are numbers of patients (\%), unless indicated otherwise.

Supplemental Table 4. Baseline characteristics in patients with and without baseline blood pressure

\begin{tabular}{|c|c|c|c|}
\hline & BP available & No baseline BP & P value \\
\hline $\mathrm{n}$ & 9774 & 658 & \\
\hline Sex (female) (\%) & $4221(43.0)$ & $284(43.0)$ & 1.000 \\
\hline Age (mean (sd)) & $71.0(8.1)$ & $71.2(7.9)$ & 0.556 \\
\hline Peripheral vascular disease (\%) & $2062(21.1)$ & $98(14.9)$ & $<0.001$ \\
\hline Hypertension (\%) & $4828(55.2)$ & $304(50.7)$ & 0.036 \\
\hline \multicolumn{4}{|l|}{ RCRI (\%) } \\
\hline High risk surgery & $4080(41.7)$ & $211(32.1)$ & $<0.001$ \\
\hline History of ischemic heart disease & $1712(17.6)$ & $84(12.8)$ & 0.002 \\
\hline History of chronic heart failure & $343(3.5)$ & $44(6.7)$ & $<0.001$ \\
\hline History of cerebrovascular disease & $1417(14.5)$ & $87(13.2)$ & 0.398 \\
\hline Elevated creatinin & $522(6.3)$ & $65(11.2)$ & $<0.001$ \\
\hline Insulin dependent diabetes & $1214(12.4)$ & $70(10.6)$ & 0.199 \\
\hline
\end{tabular}


Supplemental Table 4. Baseline characteristics in patients with and without baseline blood pressure

\begin{tabular}{|c|c|c|c|}
\hline & BP available & No baseline BP & P value \\
\hline \multicolumn{4}{|l|}{ Chronic medication (\%) } \\
\hline$\beta$ blockers & $2735(31.3)$ & $184(31.2)$ & 0.973 \\
\hline Calcium channel blockers & $1908(19.6)$ & $109(16.8)$ & 0.096 \\
\hline Angiotensin renin blockers & $3630(37.2)$ & $234(36.1)$ & 0.591 \\
\hline Statins & $3871(39.7)$ & $237(36.6)$ & 0.123 \\
\hline ASA (\%) & & & $<0.001$ \\
\hline 1 & $932(9.5)$ & $64(9.7)$ & \\
\hline 2 & $4601(47.1)$ & $306(46.5)$ & \\
\hline 3 & $2714(27.8)$ & $221(33.6)$ & \\
\hline 4 & $1527(15.6)$ & $67(10.2)$ & \\
\hline Spinal anaesthesia (\%) & 649 ( 6.6) & $50(7.6)$ & 0.383 \\
\hline Emergency surgery (\%) & $2592(26.5)$ & $408(62.0)$ & $<0.001$ \\
\hline Surgical specialty (\%) & & & $<0.001$ \\
\hline ENT \& dental & $1012(10.4)$ & $85(12.9)$ & \\
\hline General & $1962(20.1)$ & $214(32.5)$ & \\
\hline Gynaecological & $604(6.2)$ & $17(2.6)$ & \\
\hline Neurosurgery & $1493(15.3)$ & $135(20.5)$ & \\
\hline Orthopaedic & $893(9.1)$ & $51(7.8)$ & \\
\hline Thoracic & $52(0.5)$ & $0(0.0)$ & \\
\hline Urological & $571(5.8)$ & $19(2.9)$ & \\
\hline Vascular & $3077(31.5)$ & $128(19.5)$ & \\
\hline Other/unknown & $110(1.1)$ & $9(1.4)$ & \\
\hline POMI (\%) & $1306(20.8)$ & $127(40.4)$ & $<0.001$ \\
\hline Centre (UMC Utrecht) (\%) & $7356(75.3)$ & $585(88.9)$ & $<0.001$ \\
\hline
\end{tabular}

$\mathrm{BP}=$ blood pressure; RCRI = Revised Cardiac Risk Index; ASA = American Society of Anesthesiologists; $E N T$ = ear, nose throat; UMC Utrecht = University Medical Center Utrecht; POMI = postoperative myocardial injury. Values are numbers of patients (\%), unless indicated otherwise. 


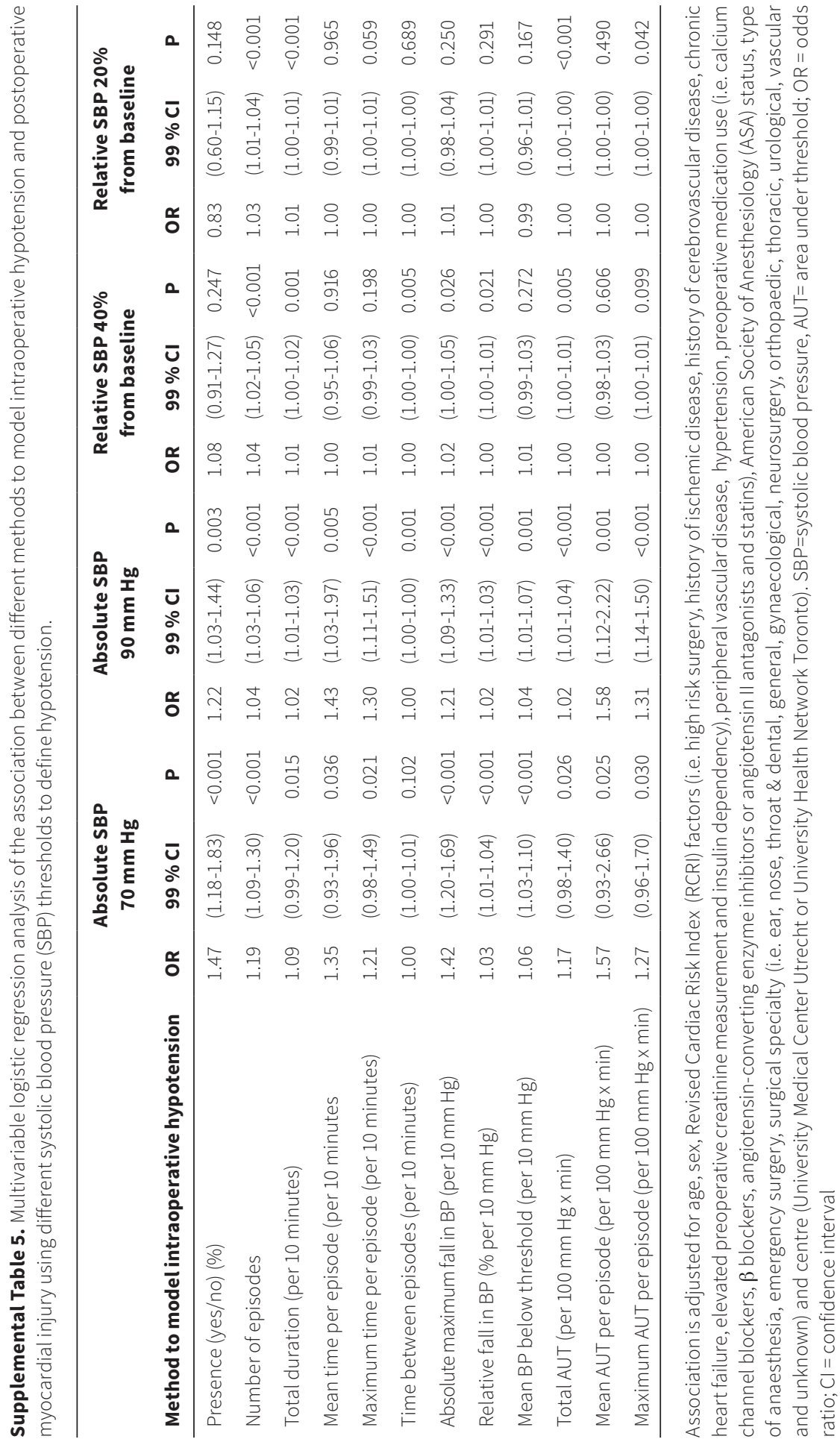




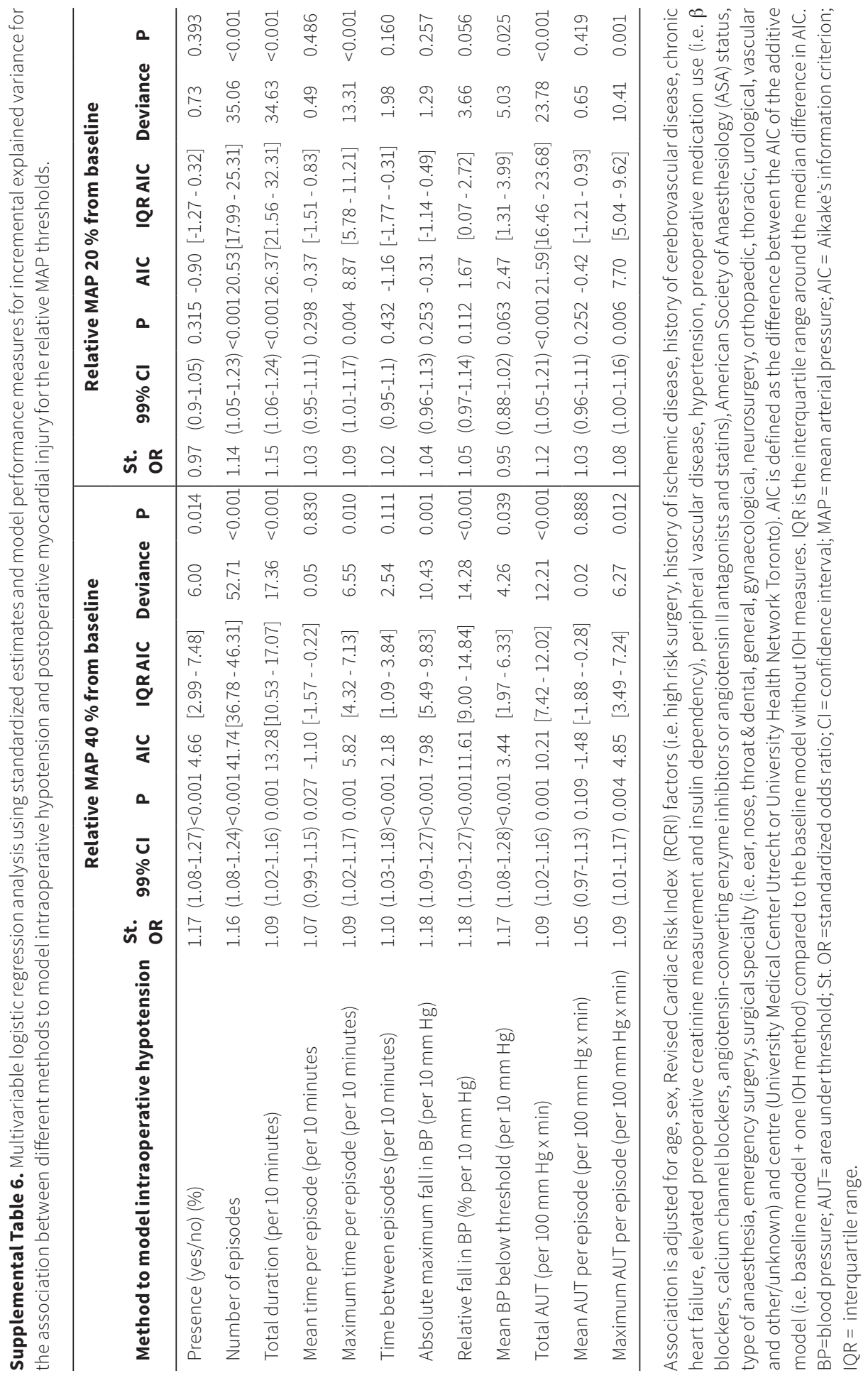




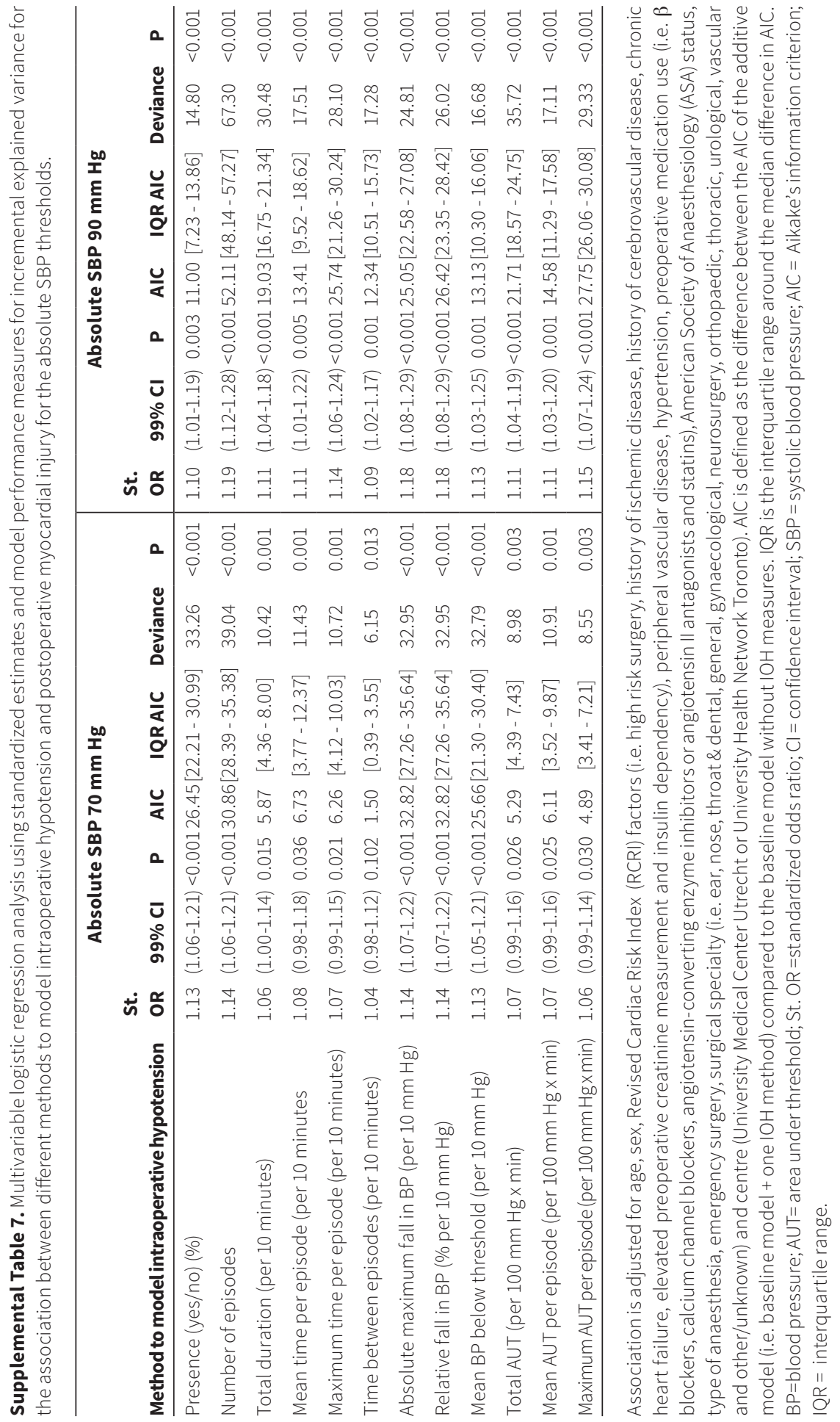




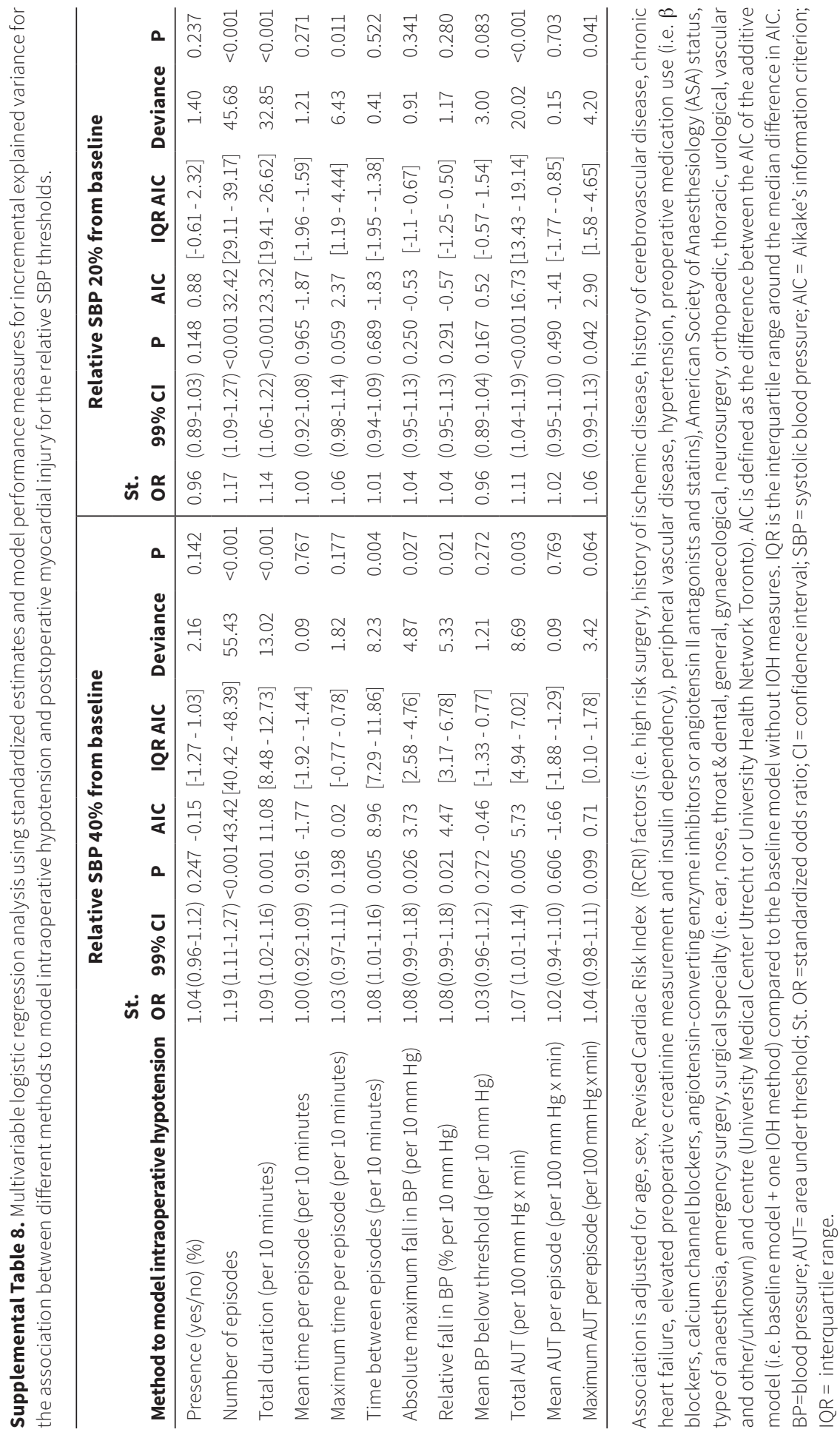


Supplemental Table 9. Baseline characteristics based on patients with or without the diagnosis of acute kidney injury (AKI) in the University Health Network Toronto cohort.

\begin{tabular}{|c|c|c|c|}
\hline & No AKI & AKI & P value \\
\hline$n$ & 2121 & 370 & \\
\hline Sex (female) (\%) & $651(31.0)$ & $115(31.0)$ & 0.93 \\
\hline Age (mean (sd)) & $70.4(9.6)$ & $71.8(10.0)$ & 0.011 \\
\hline Peripheral vascular disease (\%) & $977(46.1)$ & $178(48.1)$ & 0.502 \\
\hline Hypertension (\%) & $1589(74.9)$ & 298 ( 80.5) & 0.024 \\
\hline \multicolumn{4}{|l|}{ RCRI (\%) } \\
\hline High risk surgery & $1725(81.3)$ & $317(85.7)$ & 0.053 \\
\hline History of ischemic heart disease & $792(37.3)$ & $162(43.8)$ & 0.022 \\
\hline History of chronic heart failure & $117(5.5)$ & $42(11.4)$ & $<0.001$ \\
\hline History of cerebrovascular disease & $251(11.8)$ & $16(4.3)$ & $<0.001$ \\
\hline Elevated creatinin & $101(4.8)$ & $73(19.7)$ & $<0.001$ \\
\hline Insulin dependent diabetes & $634(29.9)$ & $135(36.5)$ & 0.013 \\
\hline \multicolumn{4}{|l|}{ Chronic medication (\%) } \\
\hline$\beta$ blockers & $635(29.9)$ & $182(49.2)$ & $<0.001$ \\
\hline Calcium channel blockers & $587(27.7)$ & $129(34.9)$ & 0.006 \\
\hline Angiotensin renin blockers & $921(43.4)$ & $127(34.3)$ & 0.001 \\
\hline Statins & $1180(55.6)$ & $190(51.4)$ & 0.141 \\
\hline ASA (\%) & & & $<0.001$ \\
\hline 1 & $6(0.3)$ & $0(0.0)$ & \\
\hline 2 & $59(2.8)$ & $8(2.2)$ & \\
\hline 3 & $833(39.3)$ & $90(24.3)$ & \\
\hline 4 & $1223(57.7)$ & $272(73.5)$ & \\
\hline Spinal anaesthesia (\%) & $135(6.4)$ & $24(6.5)$ & 1 \\
\hline Emergency surgery (\%) & $524(24.7)$ & $137(37.0)$ & $<0.001$ \\
\hline Duration of surgery in min (median [IQR]) & $195[138,269]$ & $210[133,322]$ & 0.013 \\
\hline Surgical specialty (\%) & & & 0.001 \\
\hline ENT \& Dental & $87(4.1)$ & $5(1.4)$ & \\
\hline General & $101(4.8)$ & $11(3.0)$ & \\
\hline Gynaecological & $58(2.7)$ & $1(0.3)$ & \\
\hline Thoracic & $45(2.1)$ & $7(1.9)$ & \\
\hline Urological & $141(6.6)$ & $36(9.7)$ & \\
\hline Vascular & $1680(79.2)$ & $310(83.8)$ & \\
\hline Other/unknown & $9(0.4)$ & $0(0.0)$ & \\
\hline POMI (\%) & 307 (14.5) & $154(41.6)$ & $<0.001$ \\
\hline
\end{tabular}

RCRI = Revised Cardiac Risk Index; ASA = American Society of Anesthesiologists; ENT = ear, nose throat; POMI = postoperative myocardial injury. Values are numbers of patients (\%), unless indicated otherwise. 
Supplemental Table 10. Incidence of acute kidney injury (AKI) and hypotension for different thresholds to define hypotension

\begin{tabular}{lcc}
\hline & Hypotension & $\begin{array}{c}\text { Hypotensive patients with } \\
\text { AKI }\end{array}$ \\
\hline Absolute MAP $50 \mathrm{~mm} \mathrm{Hg}(\%)$ & $439(17.6)$ & $114(26.0)$ \\
Absolute MAP 60 mm Hg (\%) & $1564(62.8)$ & $329(21.0)$ \\
Relative MAP 40\% from baseline (\%) & $1190(47.8)$ & $223(18.7)$ \\
Relative MAP 20\% from baseline (\%) & $2121(85.1)$ & $371(17.5)$ \\
Absolute SBP 70 mm Hg (\%) & $322(12.9)$ & $94(29.2)$ \\
Absolute SBP 90 mm Hg (\%) & $1767(70.9)$ & $333(18.8)$ \\
Relative SBP 40\% from baseline (\%) & $1545(62.0)$ & $278(18.0)$ \\
Relative SBP 20\% from baseline (\%) & $2201(88.4)$ & $387(17.6)$ \\
\hline
\end{tabular}

MAP = mean arterial pressure, $\mathrm{SBP}=$ systolic blood pressure, $\mathrm{n}=2,491$, incidence $\mathrm{AKI}$ in this population was $14.8 \%(n=370)$. 


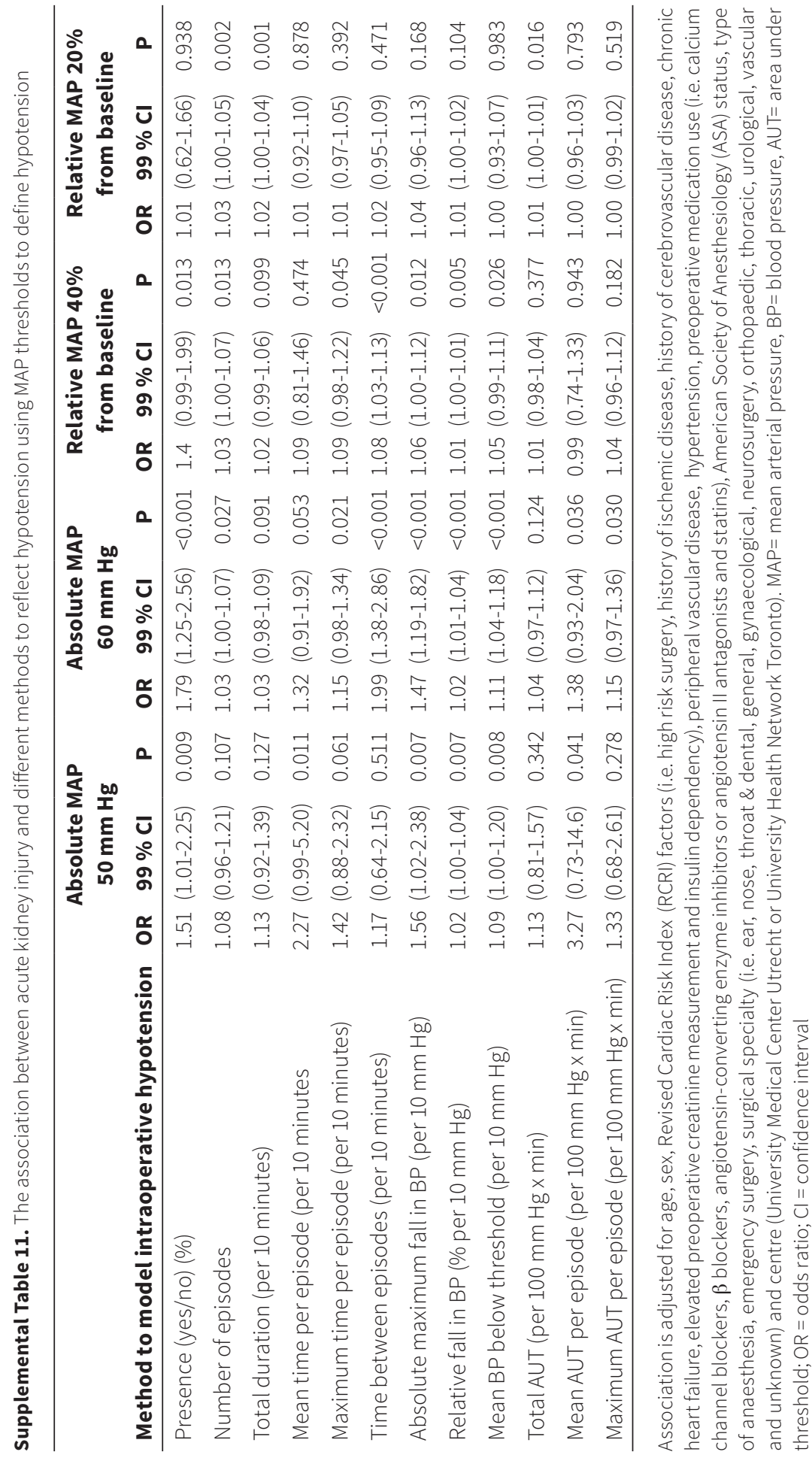




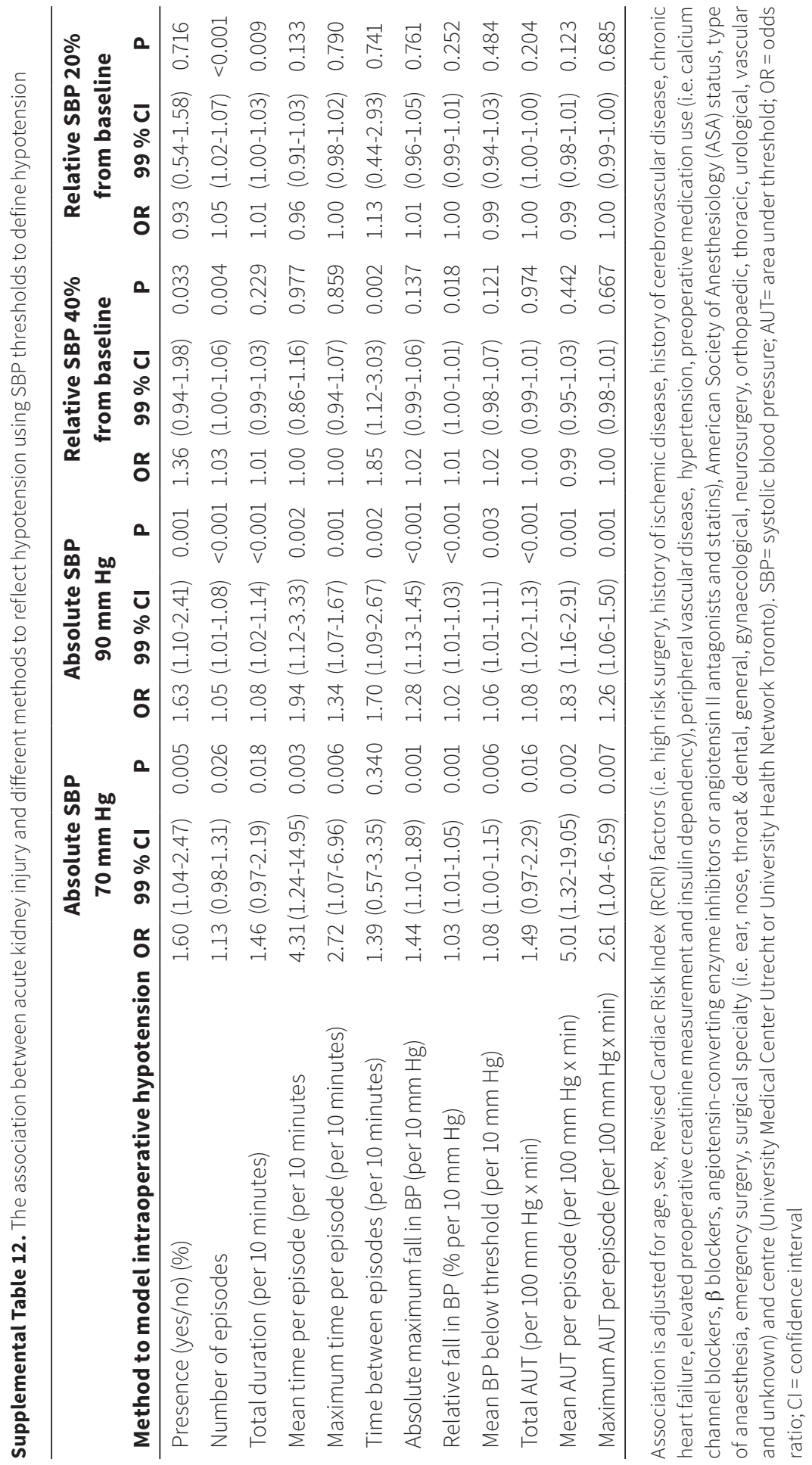




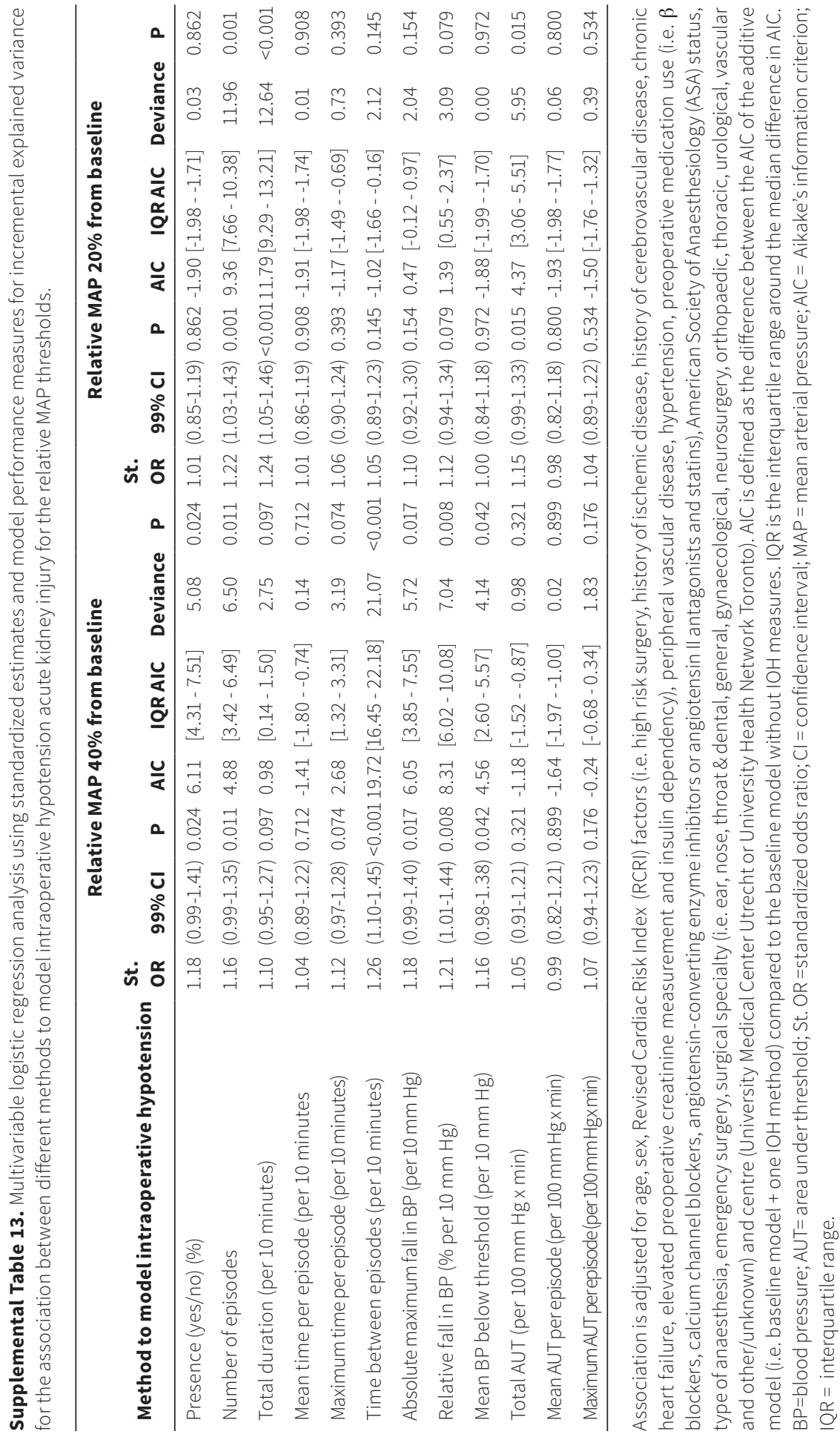




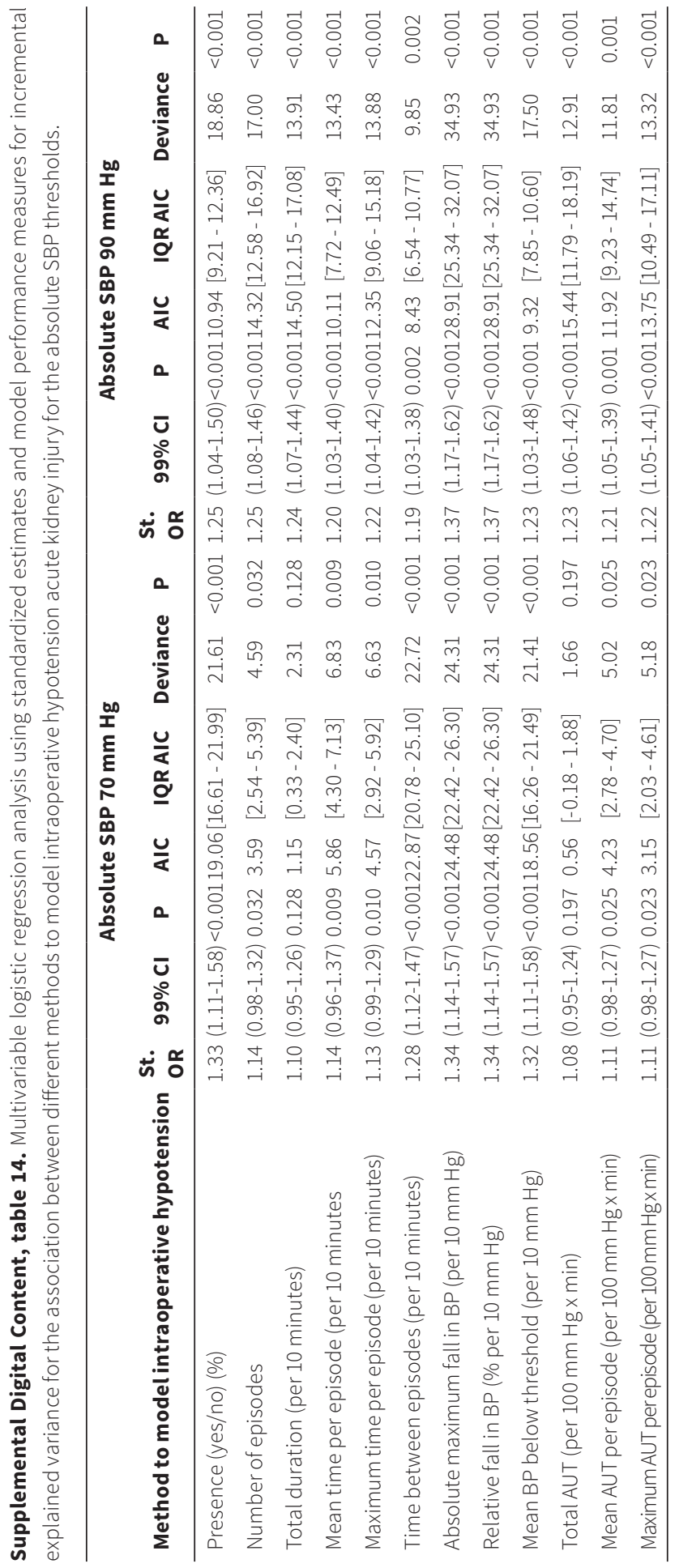

崖

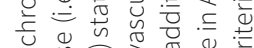

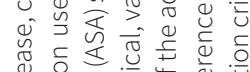

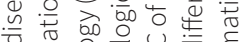

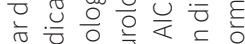

$\frac{3}{5}$ d

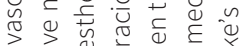

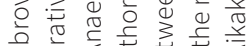

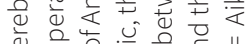

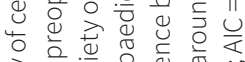

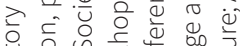

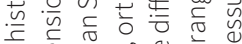

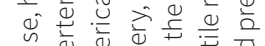

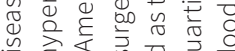

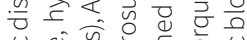

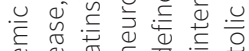

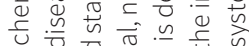

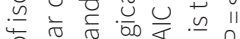

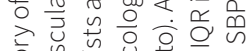

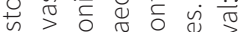

둥

त्रे ब्र

on $=ㅡ$

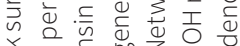

는

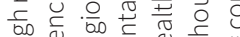

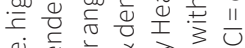

히

ज过荝

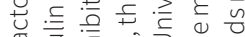

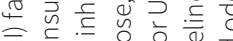

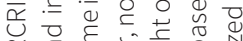

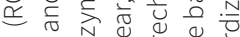

শิ

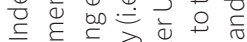

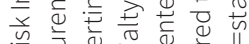

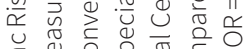

.

¿

U.

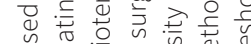

ट पू पूर

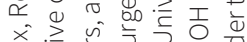

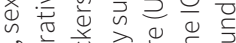

i

ए人

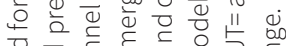

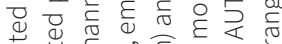

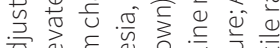

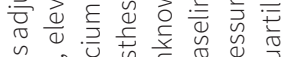

约

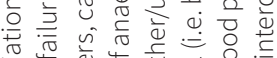

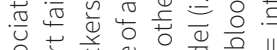

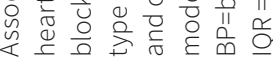




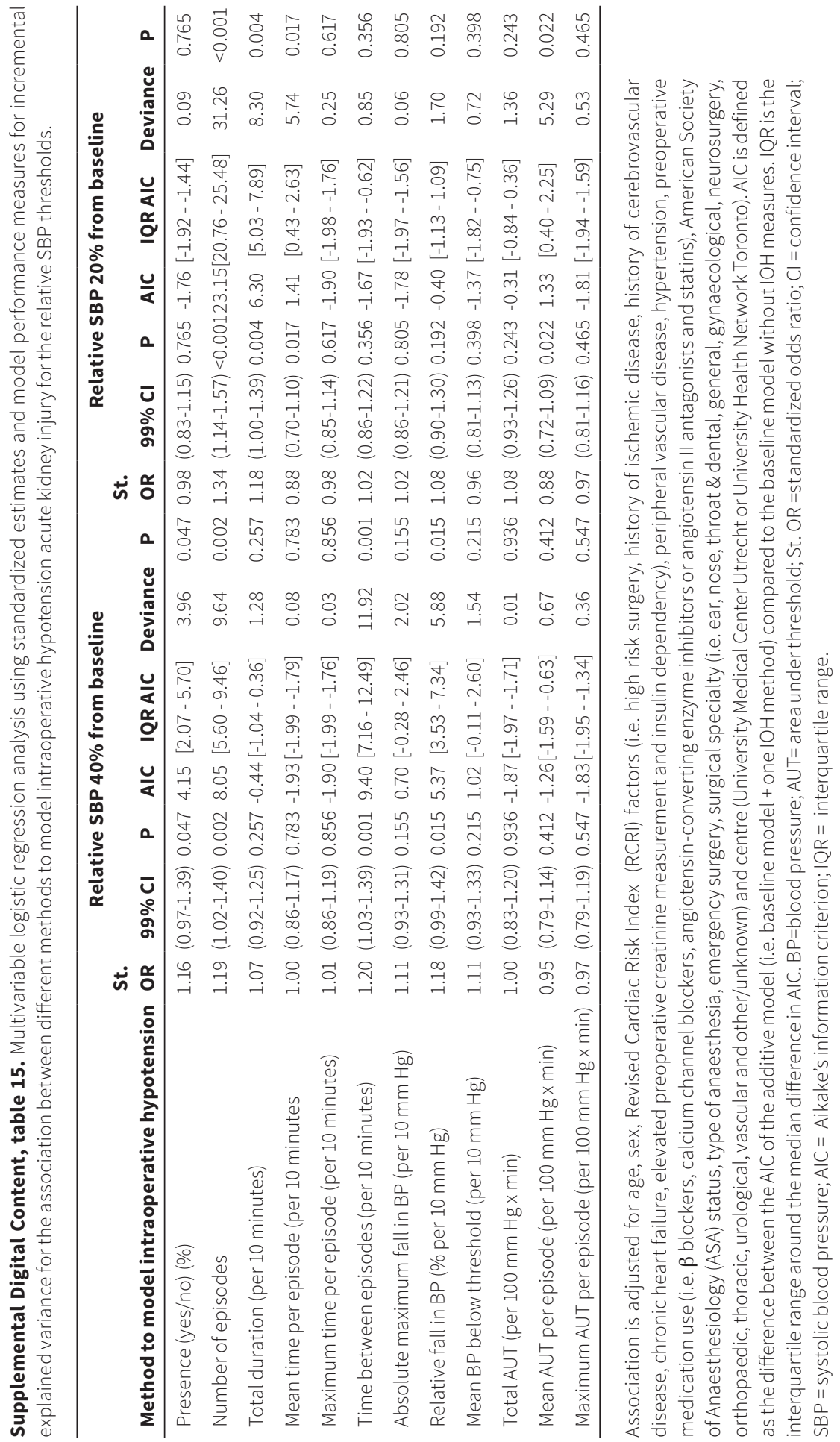




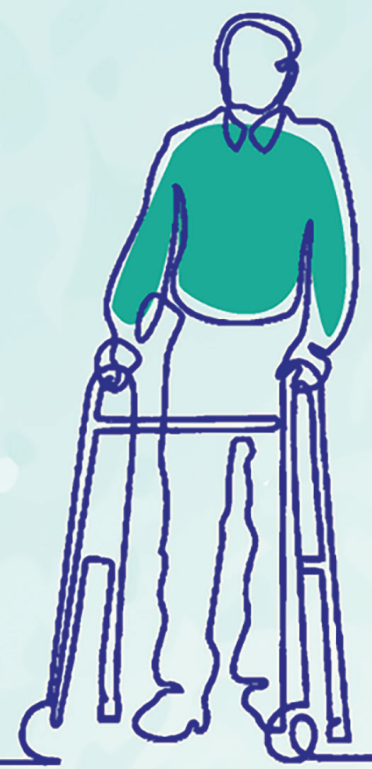




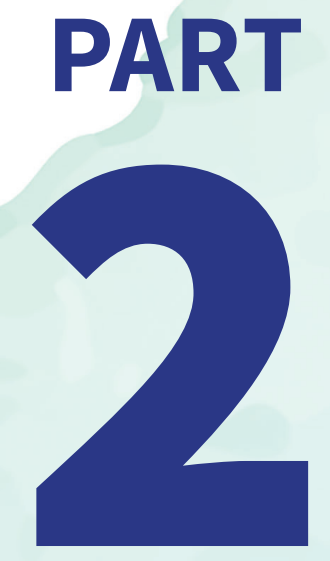

ADDED VALUE OF BIOMARKERS TO

THE RCRI 


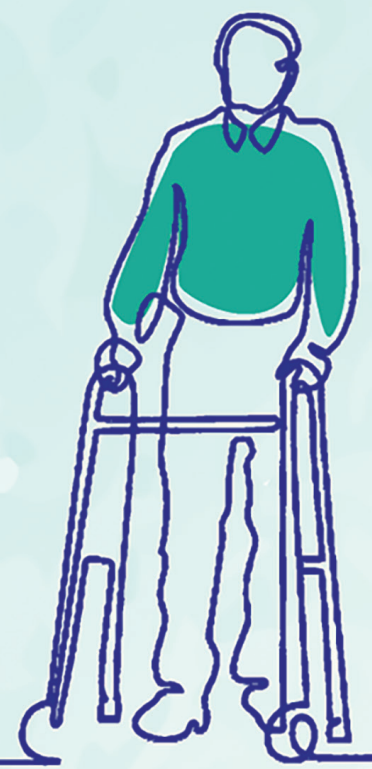




\section{CHAPTER}

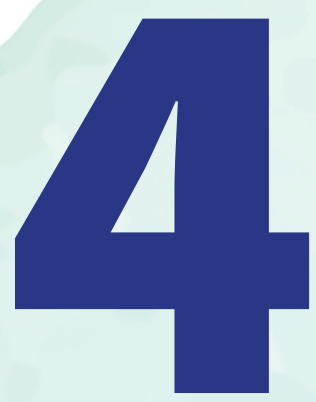

The added value of biomarkers to the Revised Cardiac Risk Index to predict major adverse cardiac events and allcause mortality after non-cardiac surgery: protocol of a systematic review and meta-analysis

Lisette M. Vernooij Johanna A. A. G. Damen

Wilton A. van Klei

Karel G.M. Moons Linda M. Peelen 


\section{BACKGROUND}

\section{Description of the condition}

Worldwide, over 300 million patients undergo intermediate to high risk non-cardiac surgery every year (1), and this number has been increasing continuously. (2) Despite the beneficial aspects of surgery, approximately 19\% of these patients will suffer an in-hospital major adverse postoperative event (MAPE; (3)). The most common MAPE had an infectious (33\%) or cardiovascular origin (19\%), with the highest mortality rates (7.0\%) observed in patients with a major adverse cardiac event (MACE; (3)). Such complications are difficult to diagnose, as typical symptoms are often not present in most postoperative patients (e.g. chest pain may be masked by pain medication). Therefore, preoperative risk stratification in these patients using available clinical information is an important component of any strategy to prevent these complications, and this has been recommended in clinical guidelines. $(4,5)$ Informing patients and physicians about perioperative risks might result in changes in patient management and optimisation before surgery by for example, performing additional diagnostic tests or interventions.

\section{Description of the prognostic model}

The Revised Cardiac Risk Index (RCRI) is a predictive tool to preoperatively estimate the postoperative probability of a MACE in patients undergoing non-cardiac surgery. (6) The RCRI contains six equally weighted predictors, including high risk surgery (suprainguinal vascular, intrathoracic, or intraperitoneal procedures), history of myocardial infarction, history of cerebrovascular disease, chronic heart failure, renal insufficiency (creatinine concentration > 177 $\mu \mathrm{mol} / \mathrm{L}(>2 \mathrm{mg} / \mathrm{dL})$ ) and insulin dependent diabetes. Notably, all six predictors were independent predictors of postoperative MACE in the derivation cohort, however both elevated creatinine and insulin dependent diabetes were not in the validation cohort. In addition, a systematic review that examined the performance of the RCRI in external validation studies concluded that the RCRI discriminated moderately well between patients at low versus high risk for predicting cardiac events after non-cardiac surgery. (7) However, the predictive ability of the RCRI for patients undergoing vascular surgery was less accurate. (7)

Several authors reported the added predictive value to the RCRI of one (or more) biomarkers to improve risk prediction. These biomarkers are among others, troponin, (8-10) N terminal pro-brain natriuretic peptide (NT-proBNP), (9-12) estimated glomerular filtration rate (eGFR), $(13,14)$ C-reactive protein (CRP), $(11,12)$ electrocardiography $(E C G),(15,16)$ and transthoracic echocardiography. (17) They all reflect different disease mechanisms. For example, NT-proBNP and transthoracic echocardiography reflect heart failure, troponin and ECG are associated with myocardial infarction and eGFR with kidney failure. 
The addition of troponin, NT-proBNP, CRP, or all three, to the RCRI seems promising for the prediction of MACE, as the predictive performance significantly improves compared to the RCRI by itself. (8-12)

As well as the addition of biomarkers to the RCRI, various studies compared the predictive ability of biomarkers alone to the RCRI to predict postoperative outcomes. The biomarkers included were among others brain natriuretic peptide (BNP), (18-21) eGFR, (19) cardiopulmonary exercise testing, (19) transthoracic echocardiography, (22) and CRP. (19) Similar to adding biomarkers to the RCRI, the predictive performance improves using biomarkers alone for postoperative risk predictions compared to the RCRI.

\section{Health outcomes}

The RCRI was originally developed to predict postoperative in-hospital MACES. MACEs are a leading cause of morbidity and mortality and occur in over 10 million patients undergoing noncardiac surgery. $(23,24)$ MACEs account for the highest postoperative mortality rates, (3) and they are associated with prolonged hospitalisation and increased medical costs. (25) A MACE is a composite outcome and includes, among others, cardiac death, myocardial infarction, cardiac arrest, arrhythmias, revascularisation and emergent coronary bypass graft surgery. However, the composition of cardiac outcomes to define MACE varies notably between different research groups and publications, (26) as there is no standardised definition of MACE existing. Although the outcome predicted in the development paper was MACE, (6) the RCRI has also been used to predict all-cause mortality in patients undergoing non-cardiac surgery. $(10,18)$

\section{Why it is important to do this review of these prognostic models}

The addition of one or multiple biomarker(s) to the RCRI for postoperative risk prediction will likely result in improved predictive accuracy, and thereby could lead to a recommendation to routinely measure these biomarker(s) preoperatively. Accordingly, routine measuring of these biomarkers will result in a better preoperative stratification of patients at high risk for a MACE, or all-cause mortality, or both, and thus better postoperative monitoring and patient management. More intensified monitoring of patients at increased postoperative risk could result in prevention of major complications, including MACE, in these patients. On the other hand, additional measuring of biomarkers in clinical care might also lead to overtreatment of patients without clinical signs and symptoms in which deviations in biomarkers were observed. Currently, the advantages and disadvantages of measuring such biomarkers in routine care are not yet fully understood. To date, several authors have reported on the added predictive value of biomarkers to the RCRI, (8-12) or compared the predictive performance of biomarkers themselves to the RCRI, (18-21) but no systematic review has been conducted on this topic yet. Therefore, the aim of this Cochrane Review is to quantify the added predictive value of one or multiple biomarker(s) to the RCRI and to 
estimate the predictive performance of biomarkers themselves compared to the RCRI to predict MACEs and all-cause mortality in patients undergoing non-cardiac surgery.

\section{OBJECTIVES}

The primary objective of this systematic review is to quantify the added predictive value of several biomarkers to the RCRI and to estimate the predictive performance of biomarkers compared to the RCRI alone to predict MACE and all-cause mortality in patients undergoing non-cardiac surgery.

Table 1. PICOTS of the review based on the CHARMS checklist $(27,28)$

\begin{tabular}{ll}
\hline Item & Answer \\
\hline Population targeted & Patients undergoing non-cardiac surgery \\
Intervention (index model) & Prognostic model ; Revised Cardiac Risk Index (RCRI) \\
& $\begin{array}{l}\text { Addition of biomarkers to the RCRI or comparison of } \\
\text { biomarkers alone to the RCRI }\end{array}$ \\
Outcome(s) to be predicted & Major adverse cardiac events (MACE) and all-cause mortality \\
Time span of the prediction & All time spans \\
\hline
\end{tabular}

\section{Investigation of sources of heterogeneity between studies}

We will assess sources of heterogeneity based on the population, outcome definitions and prediction horizons. The RCRI was originally developed for a non-cardiac, non-vascular surgical population to predict in-hospital MACEs. However, the RCRI has also been externally validated in vascular surgical patients, $(8,12)$ in which the predictive performance was found to be moderate. (7) In addition, prediction horizons vary between studies from in-hospital to long-term events (e.g. postoperative 1-year all-cause mortality). Finally, the composition of items that defines MACEs varies among different studies.

\section{METHODS}

\section{Criteria for considering studies for this review}

\section{Types of studies}

We will consider all studies regardless of study design, language or publication status for inclusion in this review.

\section{Types of participants (target population)}

We will include studies reporting on patients of all ages undergoing noncardiac surgery. 


\section{Types of prognostic models}

We will assess all studies reporting the external validation of the Revised Cardiac Risk Index (RCRI) both without the biomarkers, as well as with one or more biomarkers. The predictive accuracy of the extended RCRI model should thus be compared to the original RCRI model. In addition, we will also include studies reporting on the comparison between the predictive performance of biomarkers themselves to the RCRI alone (i.e. without model updating). We will exclude studies in which the RCRI is solely externally validated without extending the model with biomarker(s) or comparison of the predictive accuracy of biomarker(s) to the RCRI.

\section{Types of primary outcomes to be predicted}

The outcome of interest is postoperative in-hospital MACE, as was used for the original model development paper. (6) As mentioned before, the composition of MACE varies extensively among different studies, but we will assess and consider all for this review. Although the prediction horizon varies among different studies, we will not make any restrictions based on this and will select all for this review. Depending on the heterogeneity of the definition of MACE, we will conduct a meta-analysis to estimate the probability of a postoperative MACE in the extended model, for biomarkers alone and the RCRI itself.

\section{Types of secondary outcomes to be predicted}

Although the RCRI was developed to predict MACE , (6) several authors have reported the prognostic ability of the RCRI to predict all-cause mortality. $(10,18)$ Therefore, we will include all prognostic studies that report the added value of one or more biomarker(s) to the RCRI to predict all-cause mortality. Similar to the primary outcome, we will include all studies reporting on the external validation of RCRI both without the biomarkers, as well as with biomarker(s), or studies reporting on the comparison of the predictive ability of biomarkers alone to the RCRI independent of the prediction horizon. In case we encounter other outcomes predicted by the RCRI in which one or more biomarkers are added or compared to, we will include these studies as well.

\section{Search methods for identification of studies \\ Electronic searches}

We will search the following databases: Ovid MEDLINE and Ovid Embase. The search strategy will include an adjusted version of the Geersing search filter for prognostic studies. (29) We adjusted the filter to identify studies reporting on the validation or updating of prediction models, as well as the added value of variables to existing prediction models. Further, we used synonyms of the RCRI, including 'revised Goldman index' and 'Lee index'. The Geersing search filter was originally designed for searches in Ovid MEDLINE. (29) However for this review we also adapted the search strategy for use in Ovid Embase. The search strategies we will use are reported in Appendix 1. 
In addition, we will search in both ISI Web of Science and SCOPUS (1999 - current date) for articles referring to the original study that reported the development of the RCRI (Lee 1999). As the RCRI is a revised model from the Cardiac Risk Index by Goldman 1977 (30) and Detsky 1986, (31) we will include all references from these articles from 1999 onwards in the search as well. We will search online trial registries, i.e. ClinicalTrials.gov (www.clinicaltrials.gov), and the World Health Organization (WHO) International Clinical Trials Registry platform (ICTRP) for potential new studies investigating the predictive ability of biomarkers or the added value of one or multiple biomarker(s) to the RCRI (apps.who.int/trialsearch). For included studies we will search PubMed to check if there are any comments or retractions. We will also check the Retraction Watch Database for retractions of included articles (retractiondatabase.org/RetractionSearch). We will not apply any language restriction so as to reduce language bias.

\section{Searching other resources}

We will perform a cross-reference check in the retrieved articles and relevant review articles to identify other eligible articles, including the Ford 2010 (7) review.

\section{Data collection}

\section{Selection of studies}

Two review authors (JAD, LMV) will independently screen the results of the searches for eligibility on title and abstract for study selection. The same two review authors will independently retrieve and assess full reports for potentially relevant studies for inclusion and exclusion according to the above criteria using a predefined electronic spreadsheet. In case of disagreement, consensus will be achieved by involving a third independent review author (LMP). We will document study selection in a detailed flow chart based on the PRISMA guidelines. (32)

\section{Data extraction and management}

Two review authors will independently extract data from the selected articles according to the CHARMS checklist (27) (JAD, LMV). These items address potential issues regarding risk of bias and issues that may affect applicability of the results in relation to the intended use of the prediction model. A data extraction form will contain the following items.

1. General information: author, year of publication, journal, country, language.

2. Source of data: study design, prospective or retrospective data collection, derivation from routinely collected data or previous conducted study, data collected in academic or peripheral hospital.

3. Participants: eligibility and recruitment method (e.g. consecutive participants, location, number of centres, setting, inclusion and exclusion criteria), whether all patients were used for model validation, number of included patients, study dates (i.e. study period), 
surgical specialty, surgical intervention and other case mix variables, including age, sex, comorbidities and chronic medication use.

4. Outcomes to be predicted: definition of each of the items representing the composite outcome, number of individual component outcomes as part of the composite outcome, number of patients diagnosed with each of the individual component outcomes, assessor of the outcome was blinded from candidate predictors, whether candidate predictors were part of the outcome, timing of outcome occurrence or duration of follow-up.

5. Candidate predictors; RCRI predictors: definition of each of the original RCRI predictors used in the validation study, whether the number of RCRI predictors were used for risk prediction (i.e. combination of predictors is not important for risk prediction) or whether the individual items were used for risk prediction (i.e. the combination of predictors is important for risk prediction).

6. Candidate predictors; biomarkers: the number, type (i.e. biomarker derived from blood, imaging or patient characteristics) and definition of new (candidate) predictors, whether the biomarker added or compared to the RCRI, assay used for biomarker determination, optimal cut-off point in case of biomarkers, predefined cut-off point, handling of the biomarkers in the modelling (e.g. continuous, dichotomous, transformations).

7. Sample size: number of patients included in the study, number of patients with outcome of interest, number of events-per-variables.

8. Missing data: number of patients with any missing value, number of missing values for each predictor, type of missing data (e.g. missing at random, missing not at random), handling of missing data (i.e. complete-case analysis, multiple imputation, other methods).

9. Model performance: calibration (calibration plot, observed-to-expected ratio (O:E ratio), Hosmer-Lemeshow (33) with confidence interval, discrimination (c-statistic) with confidence interval, classification (sensitivity, specificity, negative and positive predictive value, net reclassification index, integrated discrimination improvement), overall measures of performance.

10. Model updating: method used for updating, performance for all different updates.

11. Results: each of the model performance measures reported for both the RCRI alone as for the extended model or biomarker to which the RCRI was compared, whether these performance measures were statistically compared (e.g. using P value, Aikake's Information Criterion (AIC)), whether the new model was statistically significantly improved in comparison with the RCRI alone.

12. Interpretation and discussion: comparison with other studies, discussion of generalisability, strengths and limitations. 
Three independent review authors (JAD, LMV, LMP) will pilot the data extraction form by extracting data from two selected articles. We will compare the extracted data and discuss potential issues to optimise the data extraction form.

\section{Assessment of risk of bias of included studies (see also PROBAST guidance)}

We will use the PROBAST-tool to assess risk of bias and applicability of individual studies. (34)

Two review authors will independently assess these studies. As reported in PROBAST, we will assess risk of bias for all models reported in the selected articles according to the following domains.

1. Patient selection
a. What study design was used?
b. Was the inclusion and exclusion of participants appropriate?
c. Was participant selection similar to the development study?

2. Predictors
a. Was the predictor definition similar for all participants and similar to the development study?
b. Are all predictors available at the intended time of prediction?

3. Outcome
a. Was the outcome definition prespecified?
b. Was the same definition and assessment used for predictors and outcomes in all patients?
c. Were outcome assessors blinded to predictor information?

4. Analysis
a. Was the number of participants with the outcome reasonable?
b. Was there appropriate handling of continuous and categorical predictors?
c. Was there appropriate evaluating of model performance measures, e.g. discrimination and calibration?

We will judge each of the domains for risk of bias (high, low, unclear). Judgement will be facilitated by signalling questions which can be answered with 'yes', 'probably yes', 'probably no' , 'no' or 'no information'. Questions answered with 'yes' indicate low risk of bias. We will judge risk of bias for each domain and for the model as a whole by using the answers of the signalling questions.

In addition, we will judge applicability of the model to the research question using the PROBASTtool. We will assess each of the selected articles on applicability using the first three domains, as data analysis is not related to the contribution of the review question. Two independent review 
authors (JAD, LMV) will assess the risk of bias using the PROBAST-tool. In case of disagreement, a third independent review author (LMP) will be involved to reach consensus.

\section{Measures of model's predictive accuracy to be extracted}

We will extract the reported predictive performance measures from the selected articles and use the recommended methodology for meta-analysing the predictive performance of prognostic models. (28) These performance measures include among others calibration, discrimination and reclassification measures. Calibration refers to the predictive accuracy of the model and indicates the extent to which expected outcomes (i.e. outcomes predicted by the prediction model) and the observed outcomes agree. (35) Calibration performance can be presented as calibration plots, calibration slopes and observed to expected ratios (O:E ratios). Discrimination refers to the ability of the prediction model to discriminate between those with and without the event. (35) The most commonly used discrimination measure is the concordance-statistics, i.e. c-statistic. Examining the added value of each biomarker to the RCRI or the biomarker itself compared to the RCRI is the primary aim of this review, in particular the delta c-statistics and the net reclassification index (NRI) are of primary interest.

\section{Dealing with missing data}

We will contact the corresponding authors to provide additional data for our analyses. In case of any non-response, we can estimate performance measures and standard errors such as the O:E ratio and c-statistic using formulas described by Debray 2017. (28) If this is impossible due to limited data, these articles will be thought to introduce serious bias. We will report this and we will explore the impact of the missing data in a sensitivity analysis.

\section{Assessment of heterogeneity}

We will investigate and discuss clinical and statistical heterogeneity based on the items mentioned in the section 'Data extraction and Management'. To assess between-study heterogeneity across the included studies, we will inspect the forest plots and compute the ${ }^{2}$-statistics and $\mathrm{Tau}^{2}$ to quantify the extent of the heterogeneity. We will report on heterogeneity and explore its causes by conducting subgroup analyses.

\section{Assessment of reporting biases}

Current guidelines recommend reporting both discrimination and calibration measures for all prognostic models. (36) However, several systematic reviews focusing on the methodological conduct and reporting of prognostic models found that these performance measures are frequently not reported. (37) Therefore, we will report the reporting deficiencies in the selected studies. Furthermore, most studies reporting on prognostic models are not prospectively registered and no protocol has been published, (38) which makes assessment of potential 
publication bias difficult. However, in case a study protocol is available, we will check articles for protocol violations.

\section{Data synthesis}

\section{Data synthesis and meta-analysis approaches}

We will provide an overview of the included articles which will be sorted on the biomarker added to the RCRI and on predicted outcome. We will present the author, publication year, number of patients included, biomarker(s) added, outcome definition, number of patients with the event, c-statistics and O:E ratios of RCRI alone and the extended model using a tabular display. We will present a similar table for the articles reporting on the comparison of the predictive accuracy of a particular biomarker to the RCRI.

If possible, we will meta-analyse articles reporting on the added value of a particular biomarker to the RCRI to predict a particular outcome (O:E ratio, (delta) c-statistic and net reclassification index (NRI)) and will report the pooled measure with the confidence intervals and prediction intervals. We will compare these to the 'pooled' RCRI alone to assess improved risk prediction for the extended model. Currently, no methodology is available on meta-analysing NRIs including handling of different thresholds for reclassification. In case of appropriate included articles, we will consider development of such methods. In addition, we will construct a random-effects model as we expect heterogeneity among the selected articles. (39) We will weight studies based on inverse variance analysis. We will performs meta-analyses through methods proposed by Debray 2017 using the meta-analysis packages in the R statistics language, which includes metafor (40), mvmeta (41), metamisc (42), and Ime4. (43) We will conduct similar analysis for 'pooled' performance measures comparing the predictive ability of the biomarkers alone compared to the RCRI alone.

\section{Subgroup analysis and investigation of heterogeneity}

We will perform the following subgroup analyses for the comparison of predictive performance measures between:

1. vascular surgical patients and non-vascular surgical patients;

2. patients undergoing elective or emergency surgery;

3. different prediction horizons, e.g. in-hospital, 30-day and long-term events;

4. patients in different age categories.

We will examine potential causes of heterogeneity by assessing case mix variation and differences in study characteristics (e.g. study design and prospective versus retrospective data collection). 
We will conduct meta-regression, if needed, to explore the cause and extent of the between-study heterogeneity. $(28,39)$

\section{Sensitivity analysis}

We will perform sensitivity analysis by excluding studies with high risk of bias (at least 4 domains to be 'high') and by excluding unpublished studies and studies with missing data.

\section{Summary of findings table}

We will present the 'Summary of findings' table using GRADE to assess the body of evidence of the included prognostic studies for both MACE and all-cause mortality. (44) We will assess the quality of evidence as being high-quality to moderate-, low- or very low-quality. Two review authors (LMV and JAD) will independently undertake grading the quality of evidence and they will reach agreement by consensus. We will judge characteristics of evidence based on considerations of ideal study design and inconsistency (i.e. variability in point estimates, extent of overlap in confidence intervals, and where point estimates lie in relation to decision thresholds), imprecision, indirectness (i.e. the studied population corresponds to their population of interest) and evidence for publication bias. (44)

\section{Sources of support}

This project was supported by the National Institute for Health Research, via Cochrane Infrastructure funding to the Heart Group. The views and opinions expressed therein are those of the authors and do not necessarily reflect those of the Systematic Reviews Programme, NIHR, NHS or the Department of Health, UK. 


\section{REFERENCES}

1. Rose J, Weiser TG, Hider P, Wilson L, Gruen RL, Bickler SW. Estimated need for surgery worldwide based on prevalence of diseases: a modelling strategy for the WHO Global Health Estimate. Lancet Glob Health. 2015;3 Suppl 2:S13-20.

2. Weiser TG, Haynes AB, Molina G, Lipsitz SR, Esquivel MM, Uribe-Leitz T, et al. Estimate of the global volume of surgery in 2012: an assessment supporting improved health outcomes. Lancet. 2015;385 Suppl 2:S11.

3. Global patient outcomes after elective surgery: prospective cohort study in 27 low-, middleand high-income countries. Br J Anaesth. 2016;117(5):601-9.

4. Fleisher LA, Fleischmann KE, Auerbach AD, Barnason SA, Beckman JA, Bozkurt B, et al. 2014 ACC/AHA guideline on perioperative cardiovascular evaluation and management of patients undergoing noncardiac surgery: a report of the American College of Cardiology/American Heart Association Task Force on Practice Guidelines. Circulation. 2014;130(24):e278-333.

5. Kristensen SD, Knuuti J, Saraste A, Anker S, Botker HE, De Hert S, et al. 2014 ESC/ESA Guidelines on non-cardiac surgery: cardiovascular assessment and management: The Joint Task Force on non-cardiac surgery: cardiovascular assessment and management of the European Society of Cardiology (ESC) and the European Society of Anaesthesiology (ESA). Eur J Anaesthesiol. 2014;31(10):517-73.

6. Lee TH, Marcantonio ER, Mangione CM, Thomas EJ, Polanczyk CA, Cook EF, et al. Derivation and prospective validation of a simple index for prediction of cardiac risk of major noncardiac surgery. Circulation. 1999;100(10):1043-9.

7. Ford MK, Beattie WS, Wijeysundera DN. Systematic Review: Prediction of Perioperative Cardiac Complications and Mortality by the Revised Cardiac Risk Index. Annals of Internal Medicine. 2010;152(1):26-W7.

8. Gillmann H-J, Meinders A, Grosshennig A, Larmann J, Buente C, Calmer S, et al. Perioperative Levels and Changes of High-Sensitivity Troponin T Are Associated With Cardiovascular Events in Vascular Surgery Patients. Critical Care Medicine. 2014;42(6):1498-506.

9. Kopec M, Duma A, Helwani MA, Brown J, Brown F, Gage BF, et al. Improving Prediction of Postoperative Myocardial Infarction With High-Sensitivity Cardiac Troponin T and NTproBNP. Anesthesia and Analgesia. 2017;124(2):398-405.

10. Weber M, Luchner A, Manfred S, Mueller C, Liebetrau C, Schlitt A, et al. Incremental value of high-sensitive troponin $\mathrm{T}$ in addition to the revised cardiac index for peri-operative risk stratification in non-cardiac surgery. European Heart Journal. 2013;34(11):853-62.

11. Choi JH, Cho DK, Song YB, Hahn JY, Choi S, Gwon HC, et al. Preoperative NT-proBNP and CRP predict perioperative major cardiovascular events in non-cardiac surgery. Heart. 2010;96(1):56-62.

12. Scrutinio D, Guido G, Guida P, Passantino A, Angiletta D, Santoro D, et al. Combined Use of High-sensitivity C-Reactive Protein and N-Terminal Pro-B-type Natriuretic Peptide for Risk Stratification of Vascular Surgery Patients. Annals of Vascular Surgery. 2014;28(6):1522-9.

13. Cywinski JB, Mascha EJ, Kurz A, Sessler DI. Estimated glomerular filtration rate better predicts 30-day mortality after non-cardiac surgery than serum creatinine: a retrospective analysis of 92,888 patients. Canadian Journal of Anesthesia-Journal Canadien D Anesthesie. 2015;62(7):745-52. 
14. Davis C, Tait G, Carroll J, Wijeysundera DN, Beattie WS. The Revised Cardiac Risk Index in the new millennium: a single-centre prospective cohort re-evaluation of the original variables in 9,519 consecutive elective surgical patients. Canadian Journal of Anesthesia-Journal Canadien D Anesthesie. 2013;60(9):855-63.

15. Noordzij PG, Boersma E, Bax JJ, Feringa HHH, Schreiner F, Schouten O, et al. Prognostic value of routine preoperative electrocardiography in patients undergoing noncardiac surgery. American Journal of Cardiology. 2006;97(7):1103-6.

16. van Klei WA, Bryson GL, Yang H, Kalkman CJ, Wells GA, Beattie WS. The value of routine preoperative electrocardiography in predicting myocardial infarction after noncardiac surgery. Annals of Surgery. 2007;246(2):165-70.

17. Rohde LE, Polanczyk CA, Goldman L, Cook EF, Lee RT, Lee TH. Usefulness of transthoracic echocardiography as a tool for risk stratification of patients undergoing major noncardiac surgery. American Journal of Cardiology. 2001;87(5):505-9.

18. Katsanos S, Babalis D, Kafkas N, Mavrogenis A, Leong D, Parissis J, et al. B-type natriuretic peptide vs. cardiac risk scores for prediction of outcome following major orthopedic surgery. Journal of Cardiovascular Medicine. 2015;16(6):465-71.

19. James S, Jhanji S, Smith A, O’Brien G, Fitzgibbon M, Pearse RM. Comparison of the prognostic accuracy of scoring systems, cardiopulmonary exercise testing, and plasma biomarkers: a single-centre observational pilot study. British Journal of Anaesthesia. 2014;112(3):491-7.

20. Mercantini P, Di Somma S, Magrini L, Nava AK, Scarinci A, La Torre M, et al. Preoperative Brain Natriuretic Peptide (BNP) Is a Better Predictor of Adverse Cardiac Events Compared to Preoperative Scoring System in Patients Who Underwent Abdominal Surgery. World Journal of Surgery. 2012;36(1):24-30.

21. Park K, Kim H-K, Kim Y-J, Cho G-Y, Kim K-H, Kim K-B, et al. Incremental prognostic value of early postoperative right ventricular systolic function in patients undergoing surgery for isolated severe tricuspid regurgitation. Heart. 2011;97(16):1319-25.

22. Park S-J, Choi J-H, Cho S-J, Chang S-A, Choi J-O, Lee S-C, et al. Comparison of Transthoracic Echocardiography With N-Terminal Pro-Brain Natriuretic Peptide as a Tool for Risk Stratification of Patients Undergoing Major Noncardiac Surgery. Korean Circulation Journal. 2011;41(9):505-11.

23. Devereaux PJ, Chan MTV, Alonso-Coello P, Walsh M, Berwanger O, Villar JC, et al. Association between postoperative troponin levels and 30-day mortality among patients undergoing noncardiac surgery. JAMA - Journal of the American Medical Association. 2012;307(21):2295304.

24. Ekeloef S, Alamili M, Devereaux PJ, Gogenur I. Troponin elevations after non-cardiac, nonvascular surgery are predictive of major adverse cardiac events and mortality: a systematic review and meta-analysis. British Journal of Anaesthesia. 2016;117(5):559-68.

25. Mackey WC, Fleisher LA, Haider S, Sheikh S, Cappelleri JC, Lee WC, et al. Perioperative myocardial ischemic injury in high-risk vascular surgery patients: incidence and clinical significance in a prospective clinical trial. J Vasc Surg. 2006;43(3):533-8.

26. Kip KE, Hollabaugh K, Marroquin OC, Williams DO. The problem with composite end points in cardiovascular studies: the story of major adverse cardiac events and percutaneous coronary intervention. J Am Coll Cardiol. 2008;51(7):701-7. 
27. Moons KG, de Groot JA, Bouwmeester W, Vergouwe Y, Mallett S, Altman DG, et al. Critical appraisal and data extraction for systematic reviews of prediction modelling studies: the CHARMS checklist. PLoS Med. 2014;11(10):e1001744.

28. Debray TP, Damen JA, Snell KI, Ensor J, Hooft L, Reitsma JB, et al. A guide to systematic review and meta-analysis of prediction model performance. Bmj. 2017;356:i6460.

29. Geersing GJ, Bouwmeester W, Zuithoff P, Spijker R, Leeflang M, Moons KG. Search filters for finding prognostic and diagnostic prediction studies in Medline to enhance systematic reviews. PLoS One. 2012;7(2):e32844.

30. Goldman L, Caldera DL, Nussbaum SR, SouthwickFS, Krogstad D, Murray B, et al. Multifactorial index of cardiac risk in noncardiac surgical procedures. N Engl J Med. 1977;297(16):845-50.

31. Detsky AS, Abrams HB, Forbath N, Scott JG, Hilliard JR. Cardiac assessment for patients undergoing noncardiac surgery. A multifactorial clinical risk index. Arch Intern Med. 1986;146(11):2131-4.

32. Moher D, Liberati A, Tetzlaff J, Altman DG. Preferred reporting items for systematic reviews and meta-analyses: the PRISMA statement. Bmj. 2009;339:b2535.

33. Hosmer DW, Hosmer T, Le Cessie S, Lemeshow S. A comparison of goodness-of-fit tests for the logistic regression model. Stat Med. 1997;16(9):965-80.

34. Wolff RF, Moons KGM, Riley RD, Whiting PF, Westwood M, Collins GS, et al. PROBAST: A Tool to Assess the Risk of Bias and Applicability of Prediction Model Studies. Ann Intern Med. 2019;170(1):51-8.

35. Steyerberg EW. Clinical Prediction Models: A Practical Approach to Development, Validation, and Updating: Springer New York; 2008.

36. Collins GS, Reitsma JB, Altman DG, Moons KG. Transparent Reporting of a multivariable prediction model for Individual Prognosis Or Diagnosis (TRIPOD). Ann Intern Med. 2015;162(10):735-6.

37. Bouwmeester W, Zuithoff NP, Mallett S, Geerlings MI, Vergouwe Y, Steyerberg EW, et al. Reporting and methods in clinical prediction research: a systematic review. PLoS Med. 2012;9(5):1-12.

38. Peat G, Riley RD, Croft P, Morley KI, Kyzas PA, Moons KG, et al. Improving the transparency of prognosis research: the role of reporting, data sharing, registration, and protocols. PLoS Med. 2014;11(7):e1001671.

39. Riley RD, Higgins JP, Deeks JJ. Interpretation of random effects meta-analyses. Bmj. 2011;342:d549.

40. Viechtbauer W. Conducting Meta-Analyses in R with the metafor Package. 2010. 2010;36(3):48.

41. Gasparrini A, Armstrong B, Kenward MG. Multivariate meta-analysis for non-linear and other multi-parameter associations. Stat Med. 2012;31(29):3821-39.

42. Debray TP, de Jong VMT. metamisc: Diagnostic and Prognostic Meta-Analysis. 2019.

43. Bates D, Mächler M, Bolker B, Walker S. Fitting Linear Mixed-Effects Models Using Ime4. 2015. 2015;67(1):48.

44. Iorio A, Spencer FA, Falavigna M, Alba C, Lang E, Burnand B, et al. Use of GRADE for assessment of evidence about prognosis: rating confidence in estimates of event rates in broad categories of patients. Bmj. 2015;350:h870. 


\section{SUPPLEMENTAL MATERIAL}

Supplemental section 1. MEDLINE Ovid search strategy

1. ("Revised Cardiac risk index" or RCRI or "Lee index" or "Lee-index" or "Lee's index" or "revised goldman index" or goldman or detsky or LCRI or RCl or "revised cardiac index" or "preoperative variable*" or "preoperative variable*" or "revised cardiac risk" or "cardiac risk factor"). ti,ab,kf.

2. Reproducibility of Results/ or calibration/ or Area Under Curve/ or Validation Studies.pt.

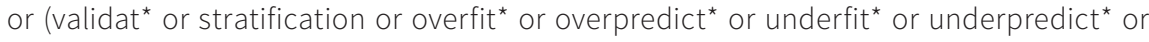
overestimation or underestimation or pooled or recalibration or re-calibration or calibration or discrimination or cohort or discriminate or c-statistic* or "c statistic*" or "Area under the curve*" or AUC or Indices or Algorithm or Multivariable or "added value" or incremental or "receiver operating curve" or roc or "receiver operating characteristic" or "c index" or "c-index" or "predictive accuracy" or "prognostic accuracy" or "reclassifi”" or "prognostic value" or "predictive value" or MACE).ti,ab,kf.

3. 1 and 2

4. (exp animals/ not humans/) or (equine or cattle or bovine or canine or mice or mouse or rat or rats or guinea-pig* or dog).ti.

5. 3 not 4 
Supplemental section 2. Ovid Embase search strategy

1. ("Revised Cardiac risk index" or RCRI or "Lee index" or "Lee-index" or "Lee's index" or "revised goldman index" or goldman or detsky or LCRI or RCI or "revised cardiac index" or "preoperative variable*" or "preoperative variable" or "revised cardiac risk" or "cardiac risk factor"”).ti,ab,kw.

2. reproducibility/orvalidation study/ or validation process/ or calibration/or area under the curve/ or (validat* or stratification or overfit* or overpredict* or underfit* or underpredict* or overestimation or underestimation or pooled or recalibration or re-calibration or calibration or discrimination or cohort or discriminate or c-statistic* or "c statistic" or "Area under the curve*" or AUC or Indices or Algorithm orMultivariable or "added value" or incremental or "receiver operating curve" or roc or "receiver operating characteristic" or "c index" or "c-index" or "predictive accuracy" or "prognostic accuracy" or "reclassifi" or "prognostic value" or "predictive value" or MACE).ti,ab,kw.

3. 1 and 2

4. ((exp experimental organism/ or animal tissue/ or animal cell/ or exp animal disease/ or exp carnivore disease/ or exp bird/ or exp experimental animal welfare/ or exp animal husbandry/ or animal behavior/ or exp animal cell culture/ or exp mammalian disease/ or exp mammal/ or exp marine species/ or nonhuman/ or animal.hw.) not human/) or (equine or cattle or bovine or canine or mice or mouse or rat or rats or guinea-pig* or dog).ti.

5. 3 not 4

6. limit 5 to (conference abstract or conference paper or "conference review")

7. 5 not 6 


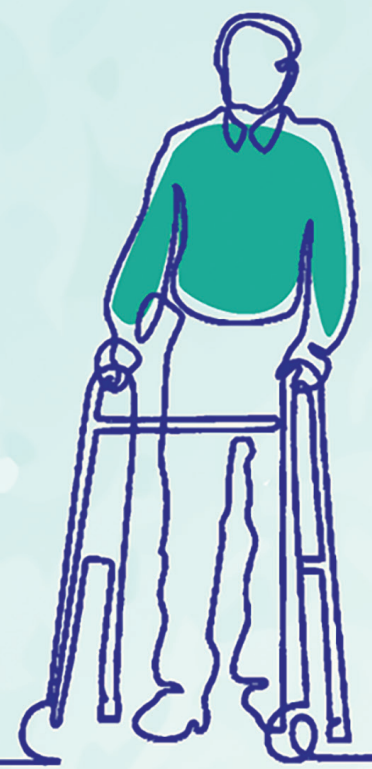




\section{CHAPTER}

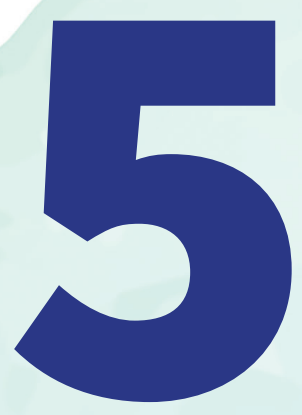

The added value of biomarkers to

the Revised Cardiac Risk Index to

preoperatively predict major adverse

cardiac events in patients who undergo

non-cardiac surgery: a systematic review

Lisette M. Vernooij

Karel G.M. Moons

Wilton A. van Klei

Toshi Takada

Judith A. van Waes

Johanna A.A.G. Damen 


\section{ABSTRACT}

Background: Approximately one in five patients undergoing surgery develops an in-hospital complication of which $19 \%$ has a cardiovascular origin with a mortality rate of $7 \%$. Preoperative risk stratification in these patients using available clinical information is an important component of any strategy to prevent such complications. The Revised Cardiac Risk index (RCRI) is a widely acknowledged and recommended prediction model to estimate preoperatively the probability of in-hospital major adverse cardiac events (MACE) in patients undergoing non-cardiac surgery. Although the RCRI is commonly used in clinical practice, its predictive performance is only moderate. As a result, numerous studies investigated whether (bio)markers added or even compared to the RCRI could improve the preoperative prediction of postoperative outcomes in patients undergoing non-cardiac surgery. However, no systematic review on the added value of (bio)markers to the RCRI has been conducted, nor on the comparative predictive performance of the RCRI versus (bio)markers or other prediction models.

Objectives: The primary objective of this systematic review was to quantify the added predictive value of (bio)markers to the RCRI to preoperatively predict the in-hospital occurrence of MACE and other adverse outcomes in patients undergoing non-cardiac surgery.

The secondary objective was to investigate the prognostic value of biomarkers as compared to the RCRI to preoperatively predict the in-hospital occurrence MACE and other adverse outcomes in patients undergoing non-cardiac surgery.

The third objective was to examine the prognostic value of other prediction models as compared to the RCRI to preoperatively predict the in-hospital occurrence of MACE and other adverse outcomes in patients undergoing non-cardiac surgery.

Search strategy: The RCRI was developed in 1999 and therefore, we searched the Ovid MEDLINE and Embase from 1 January 1999 onwards until 18 October 2018. In addition, we searched in both ISI Web of Science and SCOPUS (1 January 1999 to 18 October 2018) for articles referring to the original RCRI development study.

Selection criteria: We included all studies among adults ( $\geq 18$ years) who underwent non-cardiac surgery, reporting on the external validation of the RCRI model alone and:

- The addition of one (of more) predictor(s) to the RCRI to preoperatively predict the inhospital occurrence of MACE or other adverse outcomes.

- $\quad$ The comparison of the accuracy of (bio)markers versus that of the RCRI to preoperatively predict the in-hospital occurrence of MACE or other adverse outcomes. 
- The comparison of the accuracy of the RCRI to other models to preoperatively predict the in-hospital occurrence of MACE or other adverse outcomes.

Although MACE was the primary outcome of interest, all outcomes were considered for inclusion. There was no restriction on sex, study design, language and publication status. Studies that were published as conference proceedings only were excluded.

Data collection and analysis: We developed a data extraction form containing the items based on the CHARMS checklist. Risk of bias and concern for applicability was assessed using the Prediction model Risk of Bias Assessment Tool (PROBAST). Two review authors independently screened references for eligibility based on title and abstract. For each selected paper, full text screening, data extraction and assessment of risk of bias and concerns for applicability were performed by two independent reviewers from a team of four. Per review objective, results are presented on study characteristics, outcomes used, risk of bias and concerns for applicability, the (bio)markers and other prediction models that have been added or compared to the RCRI and - if reported the relevant predictive performance measures, such as the discriminative performance (notably c-statistic) and calibration statistics (e.g. calibration slopes or observed versus expected ratios).

Results: We screened 3437 studies resulting in a final inclusion of 84 studies. Of these, 43 articles reported on the added value of new (bio)markers or predictors to the RCRI, 44 compared the predictive performance of the RCRI to biomarkers, and 36 compared the predictive performance of the RCRI to other prediction models.

Of the 43 included articles reporting on the added value of (bio)markers to the RCRI, we identified 56 different added predictors of which 25\% were derived from blood and 34\% from imaging tests and $30 \%$ were patient characteristics. In most studies, only one predictor was added (73\%) to the RCRI, in 21\% two (21\%) and in 5\% three predictors. Biomarkers that were added to the RCRI in at least three different studies were: N-terminal pro-B type natriuretic peptide (NT-proBNP), brain natriuretic peptide (BNP), troponin and copeptin. Addition of NT-proBNP or troponin seems to improve the prediction of the RCRI model (i.e. delta c-statistic ranged from 0.04 to 0.16 and 0.06 to 0.11 for NT-proBNP and troponin, respectively). Data on the added value of BNP and copeptin was not sufficient to make any conclusions. MACE was most frequently the outcome of interest (51\%) followed by all-cause mortality (9\%) or a combination of both (14\%). The composition of predicted outcomes was very heterogeneous, especially for MACE for which 40 different definitions were reported. Also the number of included patients and outcome events was in the majority of the studies relatively low, i.e. median 455; range 122 to 2054 and median 39; range 12 to 291, respectively. 
In total, 44 studies compared the predictive performance of (bio)markers to the predictive performance of the RCRI. We identified 47 unique predictors derived from blood (34\%), imaging (34\%) or patient characteristics (39\%). Predictors for which the predictive performance was compared to the RCRI at least three times were: American Society of Anesthesiologist classification (ASA), BNP, NT-proBNP, troponin and C-reactive protein (CRP). Overall, predictions were similar between ASA and RCRI among the studied outcomes and accordingly, analyses showed no difference between both predictors. For BNP, NT-proBNP and troponin, the c-statistic was on average $0.14,0.10$ and 0.11 , respectively, higher as compared to the $\mathrm{c}$ - statistic of the RCRI alone. No difference in the predictive performance was found between the RCRI and CRP alone. In 22 studies for this objective, MACE was the outcome to be predicted followed by all-cause mortality (16\%) or a combination of both (16\%). Again, we observed large heterogeneity among the included studies due to the low number of included patients resulting in wide confidence intervals and the varying composition of outcomes.

Thirty-six articles compared the predictive ability of the RCRI to other prediction models. We identified 44 different prediction models. Thirty-one (70\%) were previously developed thus other existing prediction models, 20\% were newly developed (in that study) prediction models, and $9 \%$ of the studies updated the RCRI model and compared it to the original RCRI model. Existing prediction models that were compared to the RCRI at least three times were: ACS NSQIP score, Gupta MICA score, Goldman index and Detsky index. None of these studies showed preference for one model over the RCRI model. However, the number of outcome events was low (i.e. majority less than 30) and a wide variety in outcomes using differing compositions was predicted. The outcome of interest was most often MACE (28\%) followed by all-cause mortality (17\%) and MICA (17\%).

Authors' conclusions: We did not find a substantial added value of any (bio)marker(s) to the RCRI to preoperatively predict postoperative adverse outcomes in patients undergoing non-cardiac surgery. No difference in predictive performance was observed for the comparison of the RCRI to a single (set of) biomarker. Also, comparative models in this field do not predict better than the RCRI. If one aims to improve the predictive ability of the RCRI model to preoperatively predict in-hospital adverse outcomes, clearly more research is needed on predictors or biomarkers with similar study characteristics such as outcome, studied population and prediction horizon. Moreover, consensus on the definition of MACE is mandatory to enhance comparison between studies. 


\section{PLAIN LANGUAGE SUMMARY}

\section{Background and review question}

Although patients undergo surgery to maintain or increase life expectancy or to improve quality of life, surgery is not without risks. Approximately one out of five patients undergoing surgery will develop a postoperative complication. About $20 \%$ of these complications are related to the heart. Therefore, several tools that try to predict the chance of someone developing a heart complication before surgery using information collected in the preoperative period are available. The Revised Cardiac Risk index (RCRI) is such a tool that tries to preoperatively estimate the chance of developing a heart complication before during hospital admission in patients undergoing surgery other than heart surgery. This tool is commonly used by physicians, but the predictions are only moderate. Therefore, several researchers attempted to improve these predictions by adding extra information to this tool. This information can be derived from biomarkers, which are for example measurements from blood, imaging techniques or patient characteristics (for example, age, smoking status or physical condition). Other researchers have compared predictions of biomarkers alone to the RCRI or compared other tools to the RCRI to investigate whether this might improve predictions.

The aim of this systematic review was to investigate whether addition of biomarkers to the RCRI improves predictions of heart related complications made before surgery in patients undergoing surgery other than heart surgery. In addition, we investigated whether biomarkers and other prediction tools resulted in better predictions of heart related complications during hospitalization compared to predictions of the RCRI in patients undergoing surgery other than heart surgery.

\section{Study characteristics}

We searched for articles until 18 October 2018 and found 84 publications. Of these, 43 articles reported on the added value of biomarkers to the RCRI to improve predictions of heart related complications during hospital admission. There were 44 articles comparing predictions of the RCRI to predictions of biomarkers alone. Finally, we also identified 36 articles that compared predictions of the RCRI to other prediction tools.

\section{Key results}

Of the 43 articles that did research on whether addition of information from biomarkers to the RCRI could improve predictions of complications including heart related complications, we identified 56 different unique biomarkers that were added. Biomarkers were derived from blood in 25\% and from imaging in 34\% of the cases. Patient characteristics were added by $39 \%$ of the articles to the RCRI to improve predictions. We identified four biomarkers derived from blood that 
were investigated in at least three different articles. These were N-terminal pro-B type natriuretic peptide (NT-proBNP), brain natriuretic peptide (BNP), troponin and copeptin. NT-proBNP and BNP are biomarkers related to heart failure. Troponin is a marker that indicates injury of the heart. A high elevation of this biomarker is observed when someone gets a heart attack. Copeptin regulates among others blood volume and blood pressure. Addition of NT-proBNP or troponin seems to improve the predictions of cardiac related complications. However, the studies were not comparable due to different types of heart related complications that were predicted and the number of included patients was not sufficient to make any strong conclusions. No conclusions could be drawn for BNP and copeptin.

We identified 47 unique biomarkers derived from blood (34\%), imaging (34\%) or patient characteristics (39\%) that compared their predictions of heart related complications made before surgery to the RCRI. Biomarkers that were researched in at least three articles were American Society of Anesthesiologist classification (ASA), BNP, NT-proBNP, troponin and C-reactive protein (CRP). ASA is a tool used by anesthesia providers in every surgical patient to classify a patient's physical fitness before surgery and CRP is a biomarker derived from blood, which is elevated in case of an infection. For BNP, NT-proBNP and troponin, predictions seemed to be better for the biomarker alone compared to the RCRI. However, the number of included patients was low and predictions were mainly made for different types of heart related complications, making comparisons between different studies difficult. Predictions for either ASA or CRP were not better than the RCRI.

We found 36 articles that compared the predictions of the RCRI to other prediction tools. There were four prediction tools that were compared to the RCRI in at least three different studies. However, none of these prediction tools showed preferences in terms of better predictions for one tool over the RCRI.

\section{Authors' conclusions}

We identified 84 articles that aimed to improve the RCRI by adding (bio)markers or comparing predictions of other prediction tools or biomarkers to enhance prediction of heart related complications in patients undergoing surgery other than heart surgery. We did not find biomarker(s) that had added value to the RCRI in predicting outcomes. In addition, no other prediction tools resulted in better predictions compared to the RCRI. This means that this systematic review is inconclusive and further research is needed on the added value of (bio) markers to improve risk prediction using the RCRI. When performing such studies, researchers should take into account the health care resources, such as costs of biomarkers, the time to measure this biomarker and whether a measurement is invasive to the patient or not. In addition, there should be one definition of combination of heart related complications to be able to compare studies and thereby, providing stronger evidence. 


\section{BACKGROUND}

\section{Description of the condition}

Worldwide, over 300 million patients undergo intermediate to high risk non-cardiac surgery every year (1) and this number has been increasing continuously. (2) Despite the beneficial aspects of surgery, approximately $19 \%$ of these patients will suffer a postoperative in-hospital major adverse event. (3) The most common complications are infectious (33\%) or have a cardiovascular origin (19\%), with highest mortality rates in the latter (7\%). However, such complications are hard to diagnose, as typical symptoms are often not present in most postoperative patients (e.g., chest pain may be masked by pain medication). Therefore, preoperative risk stratification of these patients using available clinical information is an important component of any strategy to prevent these complications and has been recommended by clinical guidelines. $(4,5)$ Informing patients and physicians about their perioperative risks enhances patient management and optimization before surgery by e.g. performing additional diagnostic tests or interventions aimed at preventing postoperative complications.

\section{Description of the prognostic model}

The Revised Cardiac Risk index (RCRI) is a prognostic model to preoperatively estimate the postoperative probability of in-hospital major adverse cardiac events (MACE). (6) The RCRI is specifically developed for patients undergoing non-cardiac surgery and contains six equally weighted predictors including high risk surgery (i.e. suprainguinal vascular, intrathoracic, or intraperitoneal procedures), history of ischemic heart disease, history of cerebrovascular disease, chronic heart failure, renal insufficiency (creatinine concentration $>177 \mu \mathrm{mol} / \mathrm{L}[>2 \mathrm{mg} / \mathrm{dL}]$ ) and insulin dependent diabetes (See Table 1). Although the RCRI has been published over two decades ago, it is still commonly recommended and used in daily clinical practice $(4,5)$ as the predictors are easy to collect and calculation of the score and probability convenient. A systematic review that examined the performance of the RCRI in external validation studies concluded that the RCRI discriminated moderately well between patients at low versus high risk for predicting cardiac events after non-cardiac surgery. (7) The predictive ability of the RCRI for patients undergoing vascular surgery was less accurate. (7)

To improve the predictive performance of the RCRI, the added value of different (bio)markers to the RCRI has been extensively studied in recent years. These biomarkers could be originating from blood, such as troponin (8, 9), (NT-pro)brain natriuretic peptide (BNP) $(10,11)$ and C-reactive protein (CRP) $(10,11)$. Besides biomarkers derived from blood, the added value of imaging markers to the RCRI has also been reported including electrocardiography $(12,13)$ and coronary computed tomographic angiography. (14) Addition of new (bio)markers to the RCRI seems to improve the predictive performance of the RCRI. (8-11) 
Besides the addition of new markers to the RCRI, various studies compared the predictive ability of biomarkers to the RCRI. Again, the biomarkers compared were most commonly derived from blood such as (NT-pro) BNP $(15,16)$, troponin (17), and from imaging such as thoracic echocardiography (18).

Finally, other prediction models, such as the ACS NSQIP model (19-21) and the Gupta MICA model $(21,22)$, have also been compared on their predictive ability to that of the RCRI. (15-17)

Table 1. Scoring of the Revised Cardiac Risk Index

\begin{tabular}{|c|c|c|}
\hline Predictor & Definition & $\begin{array}{c}\text { Point } \\
\text { distribution }\end{array}$ \\
\hline High risk surgery & $\begin{array}{c}\text { Intraperitoneal, intrathoracic, or suprainguinal } \\
\text { vascular surgery }\end{array}$ & 1 \\
\hline Ischemic heart disease & $\begin{array}{l}\text { History of myocardial infarction, positive } \\
\text { exercise test, current complaint of ischemic } \\
\text { chest pain or use of nitrate therapy, or ECG with } \\
\text { Q waves. Patients with prior CABG surgery or } \\
\text { PTCA were included in this definition only if they } \\
\text { had current complaints of chest pain that were } \\
\text { presumed to be due to ischemia. }\end{array}$ & 1 \\
\hline $\begin{array}{l}\text { History of congestive } \\
\text { heart failure }\end{array}$ & $\begin{array}{l}\text { History of congestive heart failure, pulmonary } \\
\text { edema, or paroxysmal nocturnal dyspnea, } \\
\text { physical examination showing bilateral rales } \\
\text { or } \$ 3 \text { gallop, or chest radiograph showing } \\
\text { pulmonary vascular redistribution. }\end{array}$ & 1 \\
\hline $\begin{array}{l}\text { History of } \\
\text { cerebrovascular disease }\end{array}$ & History of transient ischemic attack or stroke & 1 \\
\hline $\begin{array}{l}\text { Insulin therapy for } \\
\text { diabetes mellitus }\end{array}$ & & 1 \\
\hline $\begin{array}{l}\text { Preoperative serum } \\
\text { creatinine }>2.0 \mathrm{mg} / \mathrm{dL}\end{array}$ & & 1 \\
\hline
\end{tabular}

Complication rates in patients with none of these predictors is $0.4 \%$, with 1 point is $1.0 \%, 2$ points is $7 \%$ and 3 or more points is $11 \%$. 


\section{Health outcomes}

The RCRI has originally been developed to predict postoperative in-hospital occurrence of MACE. Annually, over 10 million patients undergoing non-cardiac surgery develop a MACE. (2, 3, 23-25) MACE is a leading cause of morbidity and mortality in this patient population. (25-27) Additionally, MACE has been associated with prolonged hospitalization and increased medical costs. (28) In the literature, MACE is most commonly used as a composite outcome and includes, among others, cardiac death, (non)fatal myocardial infarction, cardiac arrest, arrhythmias, congestive heart failure and emergent coronary bypass graft surgery. Currently, there is no standardized definition of MACE, which results in varying compositions of cardiac outcomes to define MACE among different research groups and publications. (29)

Besides the prediction of the RCRI of in-hospital MACE occurrence, several other outcomes have been studied, notably all-cause mortality $(15,17)$ and even non-cardiac complications such as sepsis, respiratory failure, renal failure, readmission, discharge to a nursing facility and so on (30-33)

\section{Why it is important to do this review}

The prevalence of multi-morbidity is high and still increasing, partly due to an aging population. (34) Elderly and multimorbid patients undergoing non-cardiac surgery are more likely to develop perioperative complications (35) meaning that preoperative risk stratification in such patients is essential to direct health care towards the most needed. Preoperative risk stratification of non-cardiac surgical patients could be performed during the pre-anasthesia outpatient clinic visit using routine measurements of (bio)markers and/or the use of prognostic models, including e.g. the RCRI and ACS NSQIP model. $(6,36)$ More intensified monitoring of non-cardiac surgery patients at increased postoperative risk of MACE or other major complications, might result in better prevention of such complications.

To date, many authors reported on the added predictive value of biomarkers to the RCRI (8-11) or compared the predictive performance of biomarkers itself or other prediction models to the RCRI $(17,18,30)$. No systematic review providing a comprehensive overview of all this evidence has been conducted.

The primary objective of this systematic review was to quantify the added predictive value of (bio) markers to the RCRI to preoperatively predict the in-hospital occurrence of MACE and adverse outcomes in patients undergoing non-cardiac surgery. Furthermore, we also aimed to investigate the prognostic value of biomarkers and other prediction models as compared to the RCRI to preoperatively predict the occurrence of in-hospital MACE and other adverse outcomes in patients undergoing non-cardiac surgery. 


\section{OBJECTIVES}

\section{Primary objective}

For this Cochrane systematic review, we investigated three objectives. The primary objective of this systematic review was to quantify the added predictive value of (bio)markers to the RCRI to preoperatively predict the in-hospital occurrence of MACE and other adverse outcomes in patients undergoing non-cardiac surgery (see Table 2 for the PICOTS).

Table 2. PICOTS for the primary objective based on the CHARMS checklist (37-39)

\begin{tabular}{|c|c|}
\hline Item & Answer \\
\hline Population & Patients undergoing non-cardiac surgery \\
\hline Index Model & Revised Cardiac Risk Index (RCRI) \\
\hline Comparator & $\begin{array}{l}\text { Addition of biomarker(s) to the RCRI; comparison of biomarkers to the RCRI; } \\
\text { comparison of the prediction models to the RCRI. }\end{array}$ \\
\hline Outcome(s) & $\begin{array}{l}\text { Postoperative occurrence of (in-hospital) major adverse cardiac events, } \\
\text { all-cause mortality and other adverse outcomes }\end{array}$ \\
\hline \multirow{2}{*}{ Timing } & Time point of prognostication: before surgery \\
\hline & Prediction horizon: all time spans \\
\hline Setting & $\begin{array}{l}\text { To inform physicians preoperatively of the patient's risk of developing } \\
\text { in-hospital events after non-cardiac surgery }\end{array}$ \\
\hline
\end{tabular}

\section{Other objectives}

The secondary objective was to investigate the prognostic value of biomarkers as compared to the RCRI to preoperatively predict the in-hospital occurrence MACE and other adverse outcomes in patients undergoing non-cardiac surgery.

The third objective was to examine the prognostic value of other prediction models as compared to the RCRI to preoperatively predict the in-hospital occurrence of MACE and other adverse outcomes in patients undergoing non-cardiac surgery.

\section{Investigation of sources of heterogeneity between studies}

The RCRI was originally developed for the preoperative prediction of in-hospital MACE in the non-cardiac, nonvascular surgical population. (6) Numerous external validations of the RCRI have been performed in various non-cardiac surgical subpopulations, such as vascular $(8,11)$ 
and orthopaedic surgical patients. $(15,40)$ Additionally, as mentioned before, the composition of MACE widely varies among different studies that validated the RCRI. Also, the RCRI has been validated for the prediction of other outcomes as well, including all-cause mortality and noncardiac complications. Various prediction horizons are also used among different RCRI validation studies. Finally, several studies used other definitions for the RCRI predictors or used unclear predictor definitions, especially for the predictors ischemic heart disease, congestive heart failure and high risk surgery. $(15,41,42)$ All these different types of heterogeneity were important reasons for this large comprehensive systematic review.

\section{METHODS}

\section{Criteria for considering studies for this review Types of studies}

All original research reports that studied the predictive accuracy of the RCRI were considered for inclusion regardless of study design, or language. Studies which were only published as conference abstracts were excluded because of the lack of sufficient information.

\section{Targeted population}

We included studies on adult ( $\geq 18$ years) patients undergoing any type of non-cardiac surgery.

\section{Targeted markers and other prediction models}

To address the three separate objectives of this reviews, we included all studies reporting on either:

- $\quad$ the addition of one or more (bio)markers or, more generic, predictor(s) to the RCRI;

- the comparison of the predictive accuracy of the RCRI model to one or more biomarker(s); - the comparison of the predictive accuracy of the RCRI model to other prognostic models.

We defined a biomarker as a characteristic that is objectively measured and evaluated as an indicator of normal biological processes, pathogenic processes, or pharmacologic responses to a therapeutic intervention. (43) In essence, this broad definition includes all predictors that have been added or compared to the RCRI, including e.g. predictors from demographics, history taking, physical examination, blood or urine measurements, imaging, and omics. Studies reporting solely on the external validation of the original RCRI were excluded from this review.

\section{Types of outcomes to be predicted}

The primary outcome of interest was in-hospital MACE, as was used for the original RCRI model development paper. (Lee et al. 1999) For this definition, we made no distinction between fatal 
and non-fatal MACE. As secondary outcomes, we included all other outcomes that were studied for the external validation of the RCRI, such as all-cause mortality, myocardial infarction and non-cardiac complications.

In addition, there is a wide variation in the prediction horizons, ranging from studies reporting on prediction of intraoperative events (44) to long term post-discharge events. (45) Altogether, we made a priori no restrictions based on the type of outcome and used prediction horizon for inclusion in this review.

\section{Search methods for identification of studies \\ Electronic searches}

The original development study of the RCRI was published in 1999. (6) Therefore, all searches started from 1999 onwards. We searched the following databases on 18 October 2018: MEDLINE and Embase (Ovid, 1 January 1999 to 18 October 2018). We used a prediction model search filter developed by Geersing et al. (46) and adjusted the filter to identify studies reporting on the validation or updating of prediction models, as well as the added value of variables to existing prediction models. The Geersing search filter was originally designed for searches in Ovid Medline (Geersing et al. 2012), however for this review we also adapted the search strategy for use in Ovid Embase. Further, we used synonyms of the RCRI, including 'revised Goldman index' and 'Lee index'. The search strategies are reported in Supplement 1 and 2.

In addition, we searched in both ISI Web of Science and SCOPUS (1 January 1999 to 18 October 2018) for articles referring to the original RCRI development study. (6) As the RCRI is a revised model of the Cardiac Risk Index by Goldman (47) and Detsky (48), all references referring to these publications were searched from 1999 onwards as well. There was no language restriction so as to reduce language bias.

\section{Data collection and analysis \\ Selection of studies}

Two review authors (JAD, LMV) independently screened the results of the searches for eligibility based on title and abstract. In case of disagreement, abstracts were included for full text screening.

In contrast with the protocol (49), selection of studies based on full text was performed in two stages. In the first step, one reviewer (LMV) assessed whether the RCRI was mentioned in the Results and/or Methods section of the article. This was done by searching for the terms 'RCRl' or often used synonyms, i.e. 'revised Goldman index' and 'Lee index', or by searching where in the report the original paper was referenced. If this was not the case, these articles were excluded. 
The remaining studies were screened for inclusion in the review, independently by two review authors from a team of four (JAD, TT, JAvW, LMV) according to the above criteria using a predefined electronic spreadsheet. Any disagreements were resolved through discussion or by involving a third reviewer (JAD or JAvW) when necessary.

\section{Data extraction and management}

Two reviewers in a team of four (JAD, TT, JAvW, LMV) independently extracted the data from the selected articles. We developed a predefined electronic data extraction form containing items based on the CHARMS checklist. (37-39) These items address potential critical appraisal issues and issues that may affect applicability of the results in relation to the intended used of the prediction model. The data extraction form first was piloted on five included articles by three reviewers (JAD, JAvW, and LMV) and subsequently updated to optimize it to the final format. In case of any disagreement, this was resolved by discussion or a third reviewer was involved to reach consensus.

Data was extracted for the following items (see Supplement 3 for a detailed data extraction list): study design, participant eligibility criteria, study dates, information about case mix (such as age, sex), outcome definition and measurement, prediction horizon, RCRI predictor definitions and measurement, predictors that were added or compared, number of participants and events, details on (handling of) missing data, and model performance in terms of calibration, discrimination, reclassification and other measures for the original and extended model and the biomarker and prediction model to which the model was compared.

\section{Assessment of risk of bias in included studies}

We used the Prediction model Risk of Bias Assessment Tool (PROBAST) for our risk of bias and applicability assessment. $(50,51)$ In short, risk of bias was assessed according to four domains, i.e. participants, predictors, outcome and analysis. For each domain, risk of bias was rated as either 'Low risk of bias', 'High risk of bias' or 'Unclear risk of bias' based on signalling questions provided by the PROBAST tool. $(50,51)$ Based on the domain level assessments, overall risk of bias and judgements per study was established as follows;

1. 'low risk of bias': for studies in which all four domains are scored as low risk of bias

2. 'high risk of bias': for studies in which at least one domain is assessed as high risk of bias

3. 'unclear risk of bias': for studies in which at least one domain is rated as 'unclear' and the other domains are scored as 'low risk of bias'.

Besides assessment of risk of bias, PROBAST also provides for judgement of applicability of retrieved studies to the review question at hand with the following response options; "low 
concern', 'high concern' or 'unclear concern' regarding applicability. A similar approach as for the risk of bias assessment holds for the overall judgement for applicability.

Risk of bias and applicability was independently assessed by two reviewers (JAD, TT, JAvW, and LMV) for each included article. Consensus was reached by discussion or in case of any disagreements; a third reviewer was involved for the final judgement.

\section{Measures of predictive performance measures to be extracted}

For all three objectives, we extracted the reported predictive performance measures from each of the selected articles including calibration, discrimination and reclassification measures, the uncertainty around these measures (e.g. standard errors or confidence intervals). Calibration indicates the extent to which the expected number of outcomes (i.e. the probability of the outcome as predicted by the prediction model) and the observed frequency of the outcome agree. $(39,52,53)$ Extracted calibration performance measures - if reported - were calibration plots, calibration slopes and observed to expected ratios ( $O$ :E ratio). Discrimination refers to the ability of the prediction model to discriminate between those with and without the outcome event. $(39,52,53)$ The most commonly used discrimination measure is the concordance-statistics, i.e. c-statistic, which was also extracted for this review. We also extracted the delta c-statistic i.e. difference between the c-statistic of the RCRI model alone versus the RCRI model added with the biomarker(s) (for objective 1) and for the comparison between biomarkers or prediction models to the RCRI (objective $2 \& 3$ ). Furthermore, reclassification measures including the integrated discrimination improvement (IDI) and the net reclassification index (NRI) were also extracted, when reported.

\section{Dealing with missing data}

In case of any missing data about the predictive performance measure of the RCRI, extended $\mathrm{RCRI}$ and other prediction models, we contacted the original investigators to provide this missing information. If the authors did not respond, we estimated the O:E ratio and c-statistic and their corresponding confidence intervals using the guidance and formulas described by Debray et al. (38)

\section{Assessment of heterogeneity}

Clinical and statistical heterogeneity was investigated based on the items mentioned in the section data extraction and data management. In particular, differences in studied surgical populations, in the composition of MACE and other predicted outcomes, and in prediction horizons were discussed within the author team. To assess between-study heterogeneity across the included studies, we inspected the forest plots of the extracted predicted performance measures. To 
further explore causes of heterogeneity, subgroup analyses were predefined (specified in further detail below the subheading 'Subgroup analysis and investigation of heterogeneity').

\section{Assessment of reporting deficiencies}

Current guidelines recommend reporting of calibration and discrimination measures for all prediction models. $(54,55)$ However, several systematic reviews focussing on the methodological conduct and reporting of prognostic models found that these performance measures are frequently not reported. (56-61) Therefore, we also evaluated which predictive performance measures were reported and which were not reported in the selected studies. Most studies reporting on prognostic models are not prospectively registered and no protocol has been published, which makes a formal assessment of potential reporting bias difficult. However, in case a study protocol was available, articles were checked on protocol violations.

\section{Data synthesis}

\section{Data synthesis and meta-analysis approaches}

An overview of all included articles was created, sorted on the biomarker added to the RCRI and on the predicted outcomes. This overview included parameters such as, publication year, type of surgery, number of patients included, biomarker(s) added and outcome definition. A similar overview was created for the articles reporting on the comparison of the predictive accuracy of one or more biomarkers to the RCRI (objective 2), and for the articles comparing the predictive performance of other prediction models to the RCRI (objective 3). We planned to perform a metaanalysis of the predictive performance (O:E ratio, C-statistic and net reclassification index) of the RCRI model across the various validation studies, and of the RCRI with the biomarker(s) added (objective 1). However this turned out not possible due to the low number of studies reporting on the added value of the same biomarker and due to the differences in included study populations and in the outcome definitions between studies.

Instead, the performance measures (c-statistic) for RCRI models extended with biomarkers that were studied in at least three studies was presented in forest plots, without presenting a pooled estimate.

Meta-analysis of the c-statistic was also planned for the studies that compared the RCRI to biomarkers alone (objective 2), in case there were at least three studies reporting on the same biomarker and with a similar outcome definition, prediction horizon and same scale on how the predictor was studied (i.e. continuous, categorical or dichotomous). As there was no set of studies fulfilling these criteria, meta-analysis of the c-statistic for objective 2 also turned out not possible. Results were therefore visualized in forest plots without presenting a pooled estimate. 
Similar to objective 1 and 2, meta-analysis of the c-statistics was not possible for the studies that compared the predictive performance of other prediction models to the RCRI. For prediction models for which the predictive performance was compare to the RCRI at least three times, forest plots were made to visualize results without presenting a pooled estimate.

Meta-analysis of the O:E ratio was planned as well, but turned out not possible due to the low number of studies reporting any calibration measures. All analyses were performed in Rstudio using the packages metafor (62) and metamisc. (63)

\section{Subgroup analysis and investigation of heterogeneity}

The following subgroup analyses were planned:

- $\quad$ vascular surgery patients versus other non-cardiac surgery patients;

- $\quad$ patients undergoing elective versus emergency surgery;

- $\quad$ different prediction horizons, e.g. in-hospital, 30-day and long-term events;

- patients in different age categories.

For the same reasons as mentioned above, meta-analysis in these subgroups was not possible. Again, we stratified the forest plots according to the subgroups based on outcome, and reported the prediction horizon in the plot. Details on surgical population and age categories are reported in the 'Description of included studies' table. Potential causes of heterogeneity were explored by assessing case mix variation and differences in study characteristics (e.g. study design and prospective versus retrospective data collection). Meta-regression to explore the cause and extent of the between-study heterogeneity was planned but turned out not possible. $(38,64)$

\section{Sensitivity analysis}

Sensitivity analyses excluding studies with high risk of bias (at least 4 domains to be 'high') and excluding unpublished studies and studies with missing data were planned but not performed due to the large heterogeneity between studies.

\section{Rating the certainty of evidence and summary of findings}

The 'Summary of findings' table using GRADE to assess was used to present the body of evidence of the included prognostic studies for both MACE and all-cause mortality. $(65,66)$ The quality of evidence was assessed as being high-quality to moderate-, low- or very low-quality. Two review authors (LMV and JAD) independently undertook grading the quality of evidence and agreement was reached by consensus. Characteristics of evidence was judged based on considerations of ideal study design and inconsistency (i.e. variability in point estimates, extent of overlap in confidence intervals, and where point estimates lie in relation to decision thresholds), imprecision, 
indirectness (i.e. the studied population corresponds to their population of interest) and evidence for publication bias. $(65,66)$

\section{RESULTS}

\section{Description of studies \\ Results of the search}

We identified a total of 3184 articles through database searching and an additional 3514 articles from citations to the development study of the RCRI (6) and the studies of Goldman and Detsky (47, 48) After removal of 2013 duplicates and 1248 studies that were published before the development study of the RCRI in 1999, we screened 3473 articles based on title and abstract, of which 942 articles were selected for full text screening. As mentioned before, full text screening was performed in two stages. In the first stage, 39 articles were excluded as the full text could not be retrieved and another 559 studies because they did not mention the RCRI in either the Methods or Results section of the article. Thereafter, the remaining 344 full text articles were assessed for eligibility resulting in the inclusion of 84 records. A list of all included articles is available in Supplement 5. Of these 84 articles, 43 articles reported on the added value of predictors to the RCRI, 44 articles compared the predictive performance of the RCRI to biomarkers and 36 articles compared the RCRI to other prediction models. Accordingly, 28 included studies reported both the added value of a certain predictor to the RCRI and compared the predictive performance of this (bio)marker/prediction model to the RCRI. For further details of our search results, see Figure 1.

\section{Included studies}

We evaluated three review objectives, i.e. 1) the added value of (bio)markers to the RCRI, 2) the comparison of the predictive performance of single (bio)markers to the RCRI and 3) the comparison of the predictive accuracy of prediction models to the RCRI for the prediction of adverse outcomes in patients undergoing non-cardiac surgery. For each objective, results on study characteristics, outcomes used, risk of bias and concern for applicability, reporting rates of predictive performance measures and a list of (bio)markers and prediction models that have been added and/or compared to the RCRI are reported. Study characteristics per included article are presented in Supplement 4. (Bio)markers or prediction models, i.e. predictors that were used in at least three separate included studies, are described in more detail. 
Figure 1. Study flow diagram
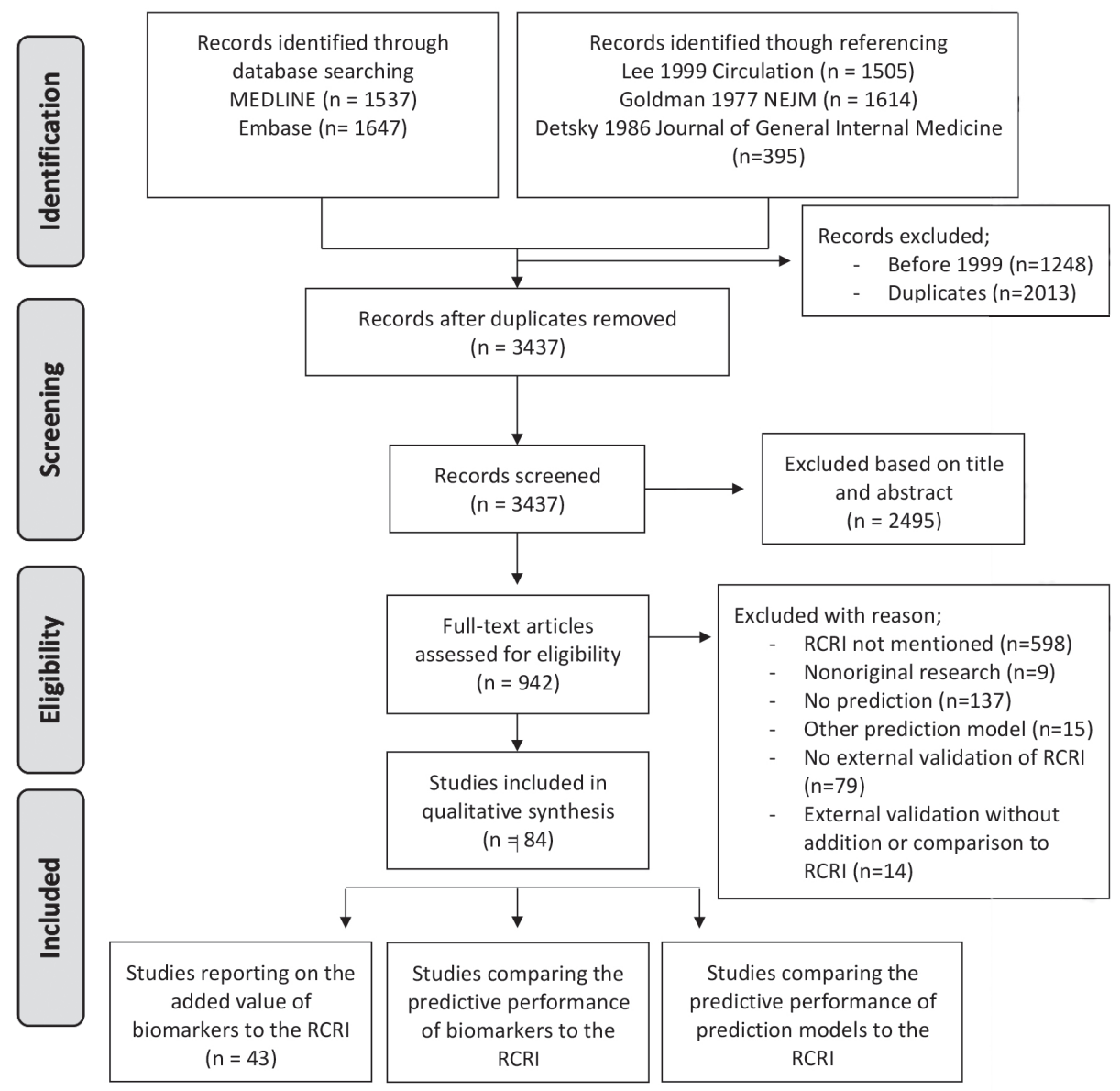


\section{Objective 1: the added predictive value of (bio)markers to the RCRI Study design and study population}

Of the 43 included articles reporting on the added value of (bio)markers to the RCRI, most studies were cohort studies $(n=39,91 \%)$ and $28(65 \%)$ had their data prospectively collected. Study participants underwent most often mixed non-cardiac surgery $(n=20,47 \%)$ followed by vascular surgery ( $n=18,42 \%$ ) (Table 3 ). In one study, the surgical specialty was not specified. Participants originated most frequently from Europe $(n=18,42 \%)$ and Asia or a combination of multiple continents (for both, $n=8,19 \%$ ). Number of included participants ranged from 77 to 108593 (median [IQR]; $467[220,1545]$ ) and the number of events ranged from 11 to 543 (39 [24, 74]). In one article, the number of events was not reported. Most frequently used prediction horizons were either during hospital admission ( $n=8,19 \%), 30$ days ( $n=23,54 \%)$ or within the first 7 days after surgery $(n=5,12 \%)$. However, there was a broad width in prediction horizons ranging from one day to four years after surgery. In terms of predicted outcomes, MACE was most frequently the outcome of interest $(n=22,51 \%)$ followed by all-cause mortality $(n=4,9 \%)$ or a combination of both $(n=6,14 \%)$. Although the RCRI was developed to predict MACE, $10(23 \%)$ articles used all-cause mortality as an outcome and one article used other complications (i.e. discharge to a nursing facility; $2.3 \%)$. The number of published articles on the added value of predictors to the RCRI increased over time with a peak in the 2012-2014 period (Figure 2).

\section{Composition of MACE}

The majority of all included articles used MACE $(n=48,57.1 \%)$ as an outcome, however its composition varied noticeably with 40 different definitions. Table 4 shows an overview of the outcome composites of MACE. For the papers reporting on the added value of (bio)markers to the RCRI, all but 3 (85\%) included myocardial infarction as one of the composites of MACE. Most definitions for MACE $(11 / 17 ; 65 \%)$ did not specify if it concerned either fatal or non-fatal myocardial infarction. Besides myocardial infarction, there was no other outcome used as a composite in more than half of the used definitions. Other frequently used included outcomes are part of MACE were heart failure (30\%), cardiac death (45\%), cardiovascular death (35\%) cardiac arrest (20\%) and pulmonary edema (25\%) (Table 4). 
Table 3. Study characteristics of included studies

\begin{tabular}{|c|c|c|c|c|}
\hline & $\begin{array}{l}\text { All included } \\
\text { studies }\end{array}$ & $\begin{array}{c}\text { Added value of } \\
\text { biomarkers }\end{array}$ & $\begin{array}{c}\text { Comparison of } \\
\text { biomarkers }\end{array}$ & $\begin{array}{c}\text { Comparison } \\
\text { of prediction } \\
\text { models }\end{array}$ \\
\hline$n$ & 84 & 43 & 44 & 36 \\
\hline \multicolumn{5}{|c|}{ Geographical area (\%) } \\
\hline Europe & $34(41.0)$ & $18(41.9)$ & $16(37.2)$ & $16(45.7)$ \\
\hline North America & $21(25.3)$ & $7(16.3)$ & $12(27.9)$ & $11(31.4)$ \\
\hline Asia & $12(14.5)$ & $8(18.6)$ & $7(16.3)$ & $2(5.7)$ \\
\hline Africa & $2(2.4)$ & $1(2.3)$ & $1(2.3)$ & $1(2.9)$ \\
\hline Australia & $1(1.2)$ & $0(0.0)$ & $1(2.3)$ & $0(0.0)$ \\
\hline South America & $3(3.6)$ & $1(2.3)$ & $1(2.3)$ & $2(5.7)$ \\
\hline Combination & $10(12.0)$ & $8(18.6)$ & $5(11.6)$ & $3(8.6)$ \\
\hline \multicolumn{5}{|l|}{ Data collection (\%) } \\
\hline Prospective & $53(63.9)$ & $28(65.1)$ & $29(65.9)$ & $22(61.1)$ \\
\hline Retrospective & $27(32.5)$ & $12(27.9)$ & $13(29.5)$ & $14(38.9)$ \\
\hline Unclear & $3(3.6)$ & $3(7.0)$ & $2(4.5)$ & $0(0.0)$ \\
\hline \multicolumn{5}{|l|}{ Study design (\%) } \\
\hline Cohort & $78(92.9)$ & $39(90.7)$ & $40(90.9)$ & $34(94.4)$ \\
\hline $\mathrm{RCT}$ & $1(1.2)$ & $1(2.3)$ & $1(2.3)$ & $1(2.8)$ \\
\hline Registry & $2(2.4)$ & $1(2.3)$ & $0(0.0)$ & $1(2.8)$ \\
\hline Other & $3(3.6)$ & $2(4.7)$ & $3(6.8)$ & $0(0.0)$ \\
\hline \multicolumn{5}{|c|}{ Surgical specialty (\%) } \\
\hline Noncardiac & $35(41.7)$ & $20(46.5)$ & $20(45.5)$ & $12(33.3)$ \\
\hline Vascular & $30(35.7)$ & $18(41.9)$ & $13(29.5)$ & $13(36.1)$ \\
\hline ENT and dental & $2(2.4)$ & $1(2.3)$ & $1(2.3)$ & $1(2.8)$ \\
\hline General & $3(3.6)$ & $0(0.0)$ & $2(4.5)$ & $2(5.6)$ \\
\hline Neurosurgery & $2(2.4)$ & $0(0.0)$ & $2(4.5)$ & $0(0.0)$ \\
\hline Orthopedic & $5(6.0)$ & $2(4.7)$ & $3(6.8)$ & $3(8.3)$ \\
\hline Other & $5(6.0)$ & $1(2.3)$ & $2(4.5)$ & $3(8.3)$ \\
\hline Not specified & $2(2.4)$ & $1(2.3)$ & $1(2.3)$ & $2(5.6)$ \\
\hline
\end{tabular}


Table 3. Study characteristics of included studies

\begin{tabular}{|c|c|c|c|c|}
\hline & $\begin{array}{l}\text { All included } \\
\text { studies }\end{array}$ & $\begin{array}{c}\text { Added value of } \\
\text { biomarkers }\end{array}$ & $\begin{array}{c}\text { Comparison of } \\
\text { biomarkers }\end{array}$ & $\begin{array}{c}\text { Comparison } \\
\text { of prediction } \\
\text { models }\end{array}$ \\
\hline$n$ & 84 & 43 & 44 & 36 \\
\hline \multicolumn{5}{|l|}{ Prediction horizon (\%) } \\
\hline Intraoperative events & $1(1.2)$ & $0(0.0)$ & $1(2.3)$ & $1(2.8)$ \\
\hline In-hospital events & $16(19.0)$ & $8(18.6)$ & $7(15.9)$ & $10(27.8)$ \\
\hline In-hospital or within & & & & \\
\hline 30 days & $5(6.0)$ & $2(4.7)$ & $2(4.5)$ & $2(5.6)$ \\
\hline $1-7$ days & $6(7.1)$ & $5(11.6)$ & $6(13.6)$ & $1(2.8)$ \\
\hline 30 days & $45(53.6)$ & $23(53.5)$ & $23(52.8)$ & $19(52.8)$ \\
\hline > 30 days (long term) & $6(7.1)$ & $3(6.9)$ & $2(4.5)$ & $2(5.6)$ \\
\hline Not reported & $3(3.6)$ & $1(2.3)$ & $2(4.5)$ & $0(0.0)$ \\
\hline \multicolumn{5}{|l|}{ Outcome } \\
\hline MACE & $39(46.4)$ & $22(51.2)$ & $22(50.0)$ & $13(36.1)$ \\
\hline $\begin{array}{l}\text { Myocardial infarction } \\
\text { and cardiac arrest }\end{array}$ & $7(8.3)$ & $2(4.7)$ & $0(0.0)$ & $6(16.7)$ \\
\hline Myocardial infarction & $4(4.8)$ & $3(7.0)$ & $2(4.5)$ & $0(0.0)$ \\
\hline $\begin{array}{l}\text { Cardiovascular } \\
\text { mortality }\end{array}$ & $2(2.4)$ & $2(4.7)$ & $0(0.0)$ & $0(0.0)$ \\
\hline Troponin elevation & $3(3.6)$ & $3(7.0)$ & $3(6.8)$ & $1(2.8)$ \\
\hline All-cause mortality & $15(17.9)$ & $4(9.3)$ & $7(15.9)$ & $10(27.8)$ \\
\hline \multicolumn{5}{|l|}{ All-cause mortality and } \\
\hline MACE & $11(13.1)$ & $6(14.0)$ & $7(15.9)$ & $4(11.1)$ \\
\hline Other & $3(3.6)$ & $1(2.3)$ & $3(6.8)$ & $2(5.6)$ \\
\hline \multicolumn{5}{|l|}{ Sample size } \\
\hline $\begin{array}{l}\text { Number of participants } \\
\text { (median [IQR]) }\end{array}$ & $\begin{array}{c}528[205 \\
1545]\end{array}$ & $467[199,955]$ & $467[199,955]$ & $855[265,2609]$ \\
\hline $\begin{array}{l}\text { Number of events } \\
\text { (median [IQR]) }\end{array}$ & $41[20,87]$ & $39[24,74]$ & $39[24,74]$ & $56[21,139]$ \\
\hline $\begin{array}{l}\text { Incidence (median } \\
[\mathrm{IQR}])\end{array}$ & $\begin{array}{c}0.08[0.04 \\
0.16]\end{array}$ & $0.09[0.06,0.17]$ & $0.09[0.06,0.17]$ & $0.06[0.04,0.17]$ \\
\hline
\end{tabular}

${ }^{*}$ RCT: randomized controlled trial; noncardiac: patients of multiple (noncardiac) surgical specialties were included in the analysis; ENT: ear, nose and throat; MACE: major adverse cardiac events; IQR: interquartile range 
Figure 2. Number of included studies over time
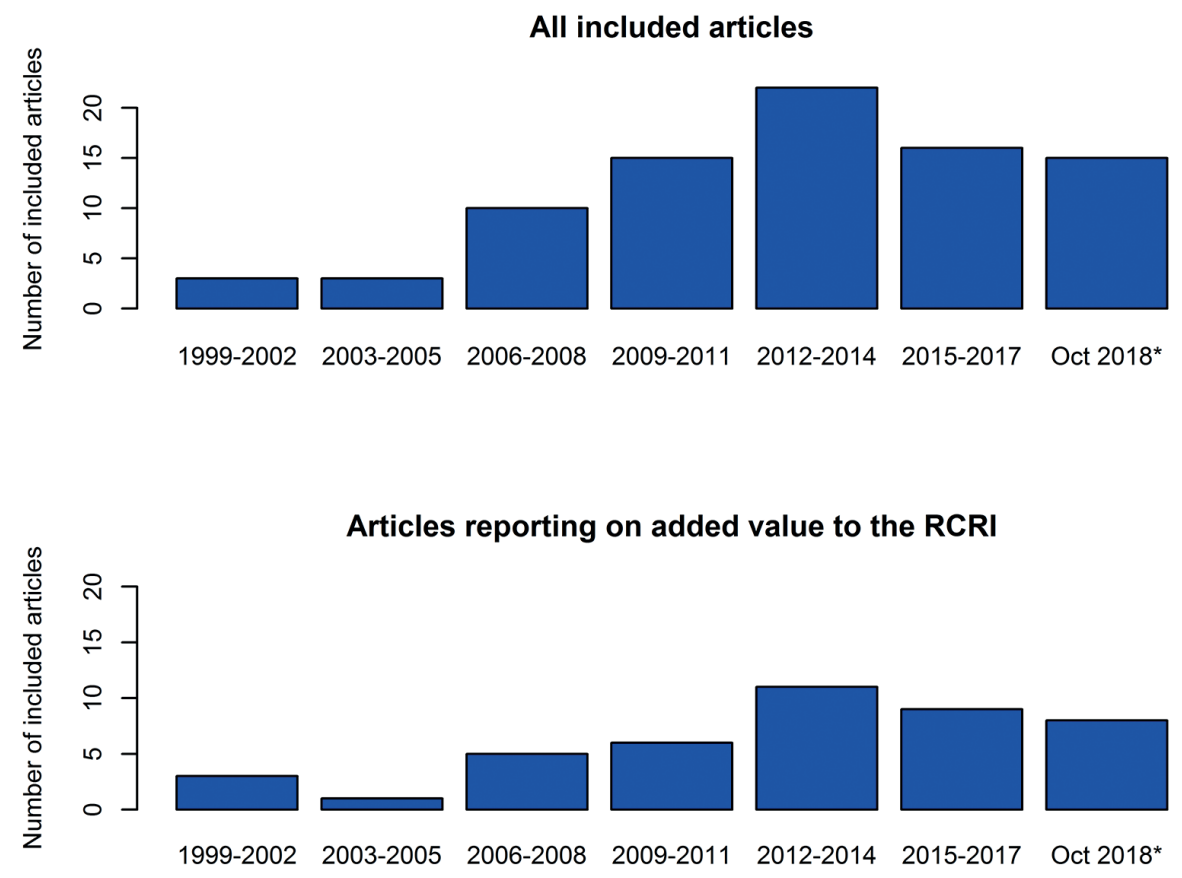

Articles reporting on the comparison of biomarkers to the RCRI

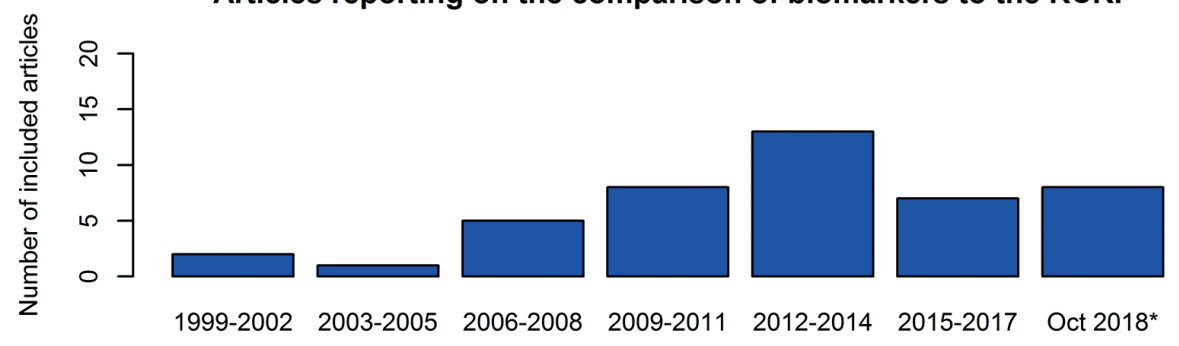

Articles reporting on the comparison of prediction models to the RCRI

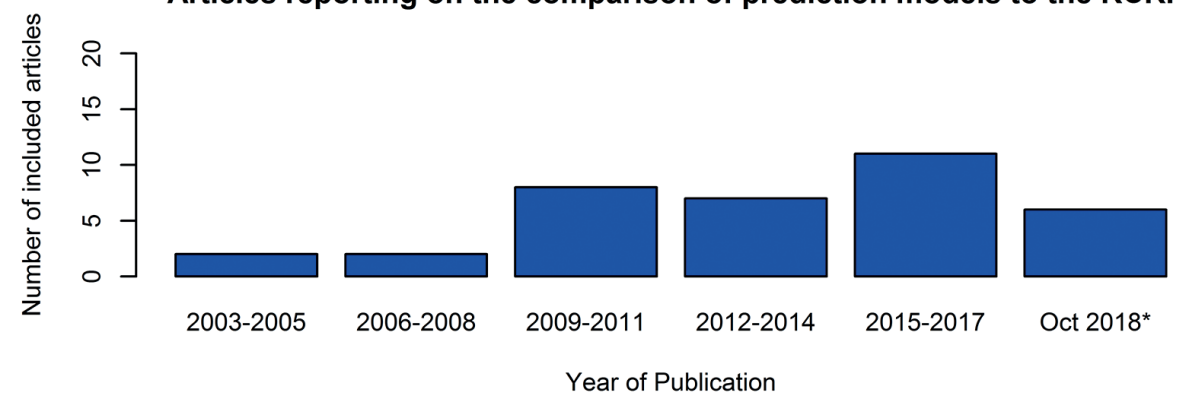

*As the search was performed on October $18^{\text {th }}$, results are shown for the period between January-October 
Table 4. Composites used to define major adverse cardiac events (MACE)

\begin{tabular}{|c|c|c|c|c|}
\hline & $\begin{array}{l}\text { All included } \\
\text { articles }\end{array}$ & $\begin{array}{c}\text { Added } \\
\text { value }\end{array}$ & $\begin{array}{l}\text { Comparison } \\
\text { biomarkers }\end{array}$ & $\begin{array}{c}\text { Comparison } \\
\text { prediction } \\
\text { models }\end{array}$ \\
\hline $\mathrm{n}$ & 40 & 20 & 21 & 13 \\
\hline Non-fatal myocardial infarction (\%) & $10(25.0)$ & $5(25.0)$ & $5(23.8)$ & $4(30.8)$ \\
\hline Fatal myocardial infarction (\%) & $1(2.5)$ & $1(5.0)$ & $1(4.8)$ & $1(7.7)$ \\
\hline Myocardial infarction, not specified (\%) & $25(62.5)$ & $11(55.0)$ & $14(66.7)$ & $7(53.8)$ \\
\hline Heart failure (\%) & $19(47.5)$ & $6(30.0)$ & $9(42.9)$ & $9(69.2)$ \\
\hline Cardiac arrest (\%) & $14(35.0)$ & $4(20.0)$ & $5(23.8)$ & $7(53.8)$ \\
\hline Cardiac death $(\%)$ & $15(37.5)$ & $9(45.0)$ & $8(38.1)$ & $5(38.5)$ \\
\hline Pulmonary edema (\%) & $10(25.0)$ & $5(25.0)$ & $5(23.8)$ & $2(15.4)$ \\
\hline Arrythmia, not specified (\%) & $10(25.0)$ & $3(15.0)$ & $6(28.6)$ & $0(0.0)$ \\
\hline (Cardio) vascular death (\%) & $7(17.5)$ & $7(35.0)$ & $3(14.3)$ & $2(15.4)$ \\
\hline Complete heart block (\%) & $6(15.0)$ & $2(10.0)$ & $3(14.3)$ & $1(7.7)$ \\
\hline Ventricular fibrillation (\%) & $5(12.5)$ & $3(15.0)$ & $5(23.8)$ & $2(15.4)$ \\
\hline Acute coronary syndrome (\%) & $5(12.5)$ & $2(10.0)$ & $2(9.5)$ & $0(0.0)$ \\
\hline Coronary revascularization (\%) & $4(10.0)$ & $2(10.0)$ & $2(9.5)$ & $2(15.4)$ \\
\hline Angina pectoris (\%) & $8(20.0)$ & $2(10.0)$ & $5(23.8)$ & $4(30.8)$ \\
\hline Myocardial injury (troponin elevation) (\%) & $4(10.0)$ & $2(10.0)$ & $4(19.0)$ & $0(0.0)$ \\
\hline Stroke (\%) & $3(7.5)$ & $0(0.0)$ & $0(0.0)$ & $2(15.4)$ \\
\hline Hypertensive crisis (\%) & $2(5.0)$ & $0(0.0)$ & $2(9.5)$ & $0(0.0)$ \\
\hline Atrium fibrillation (\%) & $1(2.5)$ & $0(0.0)$ & $1(4.8)$ & $0(0.0)$ \\
\hline Left ventricular failure (\%) & $1(2.5)$ & $0(0.0)$ & $1(4.8)$ & $1(7.7)$ \\
\hline ST-T- changes $(\%)$ & $1(2.5)$ & $0(0.0)$ & $0(0.0)$ & $1(7.7)$ \\
\hline Intraoperative hemodynamic adversity (\%) & $1(2.5)$ & $0(0.0)$ & $1(4.8)$ & $1(7.7)$ \\
\hline
\end{tabular}

* RCT: randomized controlled trial; noncardiac: patients of multiple (noncardiac) surgical specialties were included in the analysis; ENT: ear, nose and throat; MACE: major adverse cardiac events; IQR: interquartile range

\section{Risk of bias and concern for applicability}

We observed no differences in terms of assessment of risk of bias and concern for applicability among articles studying the added value of predictors or compared the predictive performance of predictors to the RCRI. Therefore, we evaluated the risk of bias and concern for applicability per domain (i.e. selection of participants, predictors, outcome and analysis) as described by the PROBAST tool $(50,51)$ for all included studies at once. 
Overall, 'risk of bias' was rated as high in at least one domain in 75 (89\%) of all included articles and in 41 (95\%) studies reporting on the added value of predictors to the RCRI. There was an overall 'high' concern of applicability in 61 (73\%) and 31 (72\%) of all included studies and studies studying on the added value of predictors to the RCRI, respectively. More detailed information is presented in Figure 3 and 4.

\section{Selection of participants}

In 59 (70 \%) included records, we judged the risk of selection bias as low. 'Risk of bias' was rated as high for 21 studies (26\%) due to inappropriate exclusion of participants (e.g. exclusion of patients with preoperative severe cardiac comorbidities, who underwent coronary revascularization or patients who were unsuitable for exercise testing) or inappropriate inclusion (e.g. only inclusion of patients who were referred to a cardiologist, had a transthoracic echocardiography or without any known cardiovascular disease). The remaining 3 studies (4\%) were rated as unclear 'risk of bias' as no eligibility criteria for inclusion in the study were described.

We judged concern for applicability for the domain 'Selection of participants' as low in 57 (68\%) of all included studies. We rated high 'concern of applicability' in 26 (29\%) records because of inclusion of patients undergoing a single procedure or with one particular comorbidity (e.g. atrium fibrillation), inclusion of very high risk patients (i.e. high incidence of comorbidities) and inclusion of patients with a either broad or small age range. The $3(4 \%)$ studies who were rated for 'unclear' risk of bias were also judged as 'unclear' concern for applicability for the same reasons.

\section{Predictors}

For the domain 'Predictors', the majority of studies ( $n=46,55 \%$ ) was rated as 'unclear' risk of bias as no information was provided on how the individual RCRI predictors were defined or measured. This was most often the case for 'history of congestive heart failure' (76\%), 'history of ischemic heart disease'(71\%) and 'history of cerebrovascular disease' (61\%). 'High' risk of bias for this domain was scored in $9(11 \%)$ articles because of different predictor definitions compared to the definitions of the development study. Differences were most often observed for the definition of 'history of ischemic heart disease' (16\%) and 'history of congestive heart failure' (17\%).

Concern for applicability was rated as 'low' in 41 (49\%) studies, as 'unclear' in 35 (42\%) and as 'high' in $8(10 \%)$ articles. Judgement was based on similar reasons as mentioned above for risk of bias.

\section{Outcome}

'High' risk of bias for the domain 'Outcome' was scored in 5 (6\%) of the included articles mostly due to inappropriate assessment of the outcome. Eighteen (21\%) studies were judged as 'unclear' risk of bias as in many studies; there was no clear outcome definition, no information on how the 
outcome was assessed or whether outcome assessors were blinded from predictor information. The remaining 61 (73\%) articles were rated as 'low' risk of bias for this domain.

The RCRI has been developed to predict postoperative in-hospital MACE. However, many studies used the RCRI for predicting other outcomes, including all-cause mortality and noncardiac complications, and therefore we judged these studies ( $n=53,63 \%$ ) as 'high' concern for applicability for this domain. Concern for applicability was scored as 'unclear' in 5 (6\%) studies due to unclear outcome definitions.

\section{Analysis}

We rated risk of bias for the domain 'Analysis' as 'high' in the majority of the included articles ( $n=70$, $83 \%)$ mainly due to low number of outcome events. The PROBAST-tool recommends at least 100 outcome events as biased estimates of model performance become more likely otherwise. $(50,51)$ Other reasons for scoring risk of bias as 'high' were dichotomization of predictors, not reporting appropriate performance measures (i.e. discrimination and/or calibration) at all or without uncertainty measures (i.e. confidence intervals or standard errors). In addition, none of the included articles using imputation for handling of missing data. Only 21 (25\%) articles reported that they did complete case analysis and the remaining articles did not mention handling of missing data. The remaining articles ( $n=14,16 \%)$ were rated as 'low' risk of bias. 
Figure 3. Risk of bias assessment based on the PROBAST-tool

All included articles
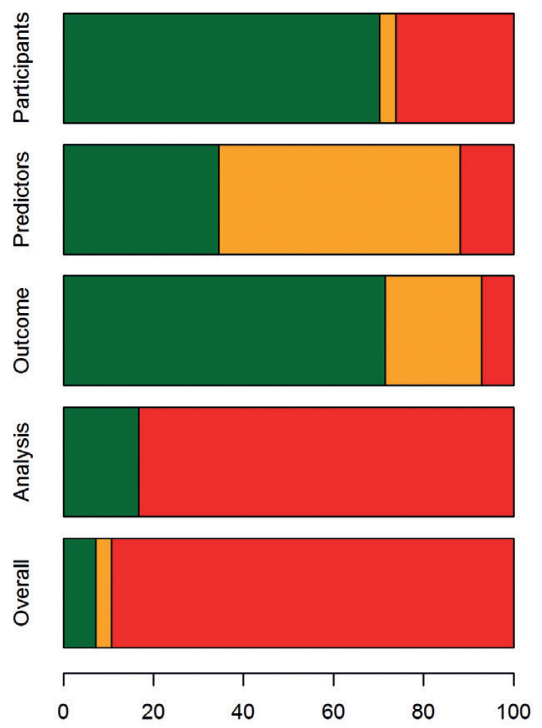

Comparing biomarkers to the RCRI
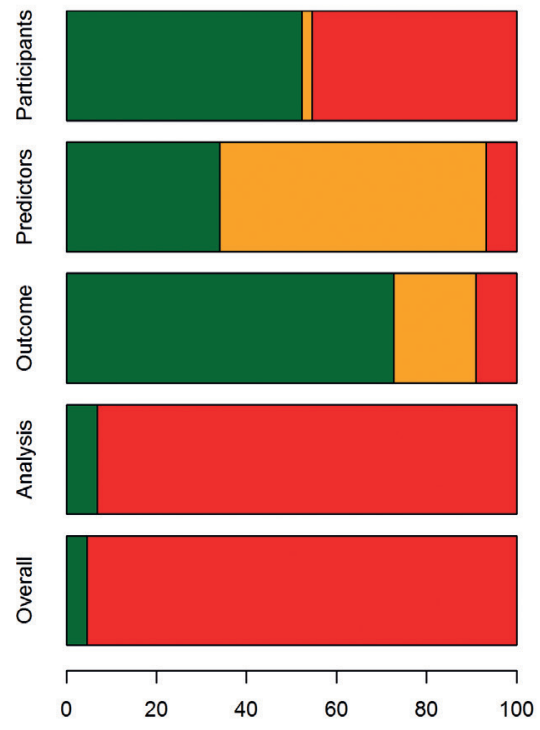

Percentage
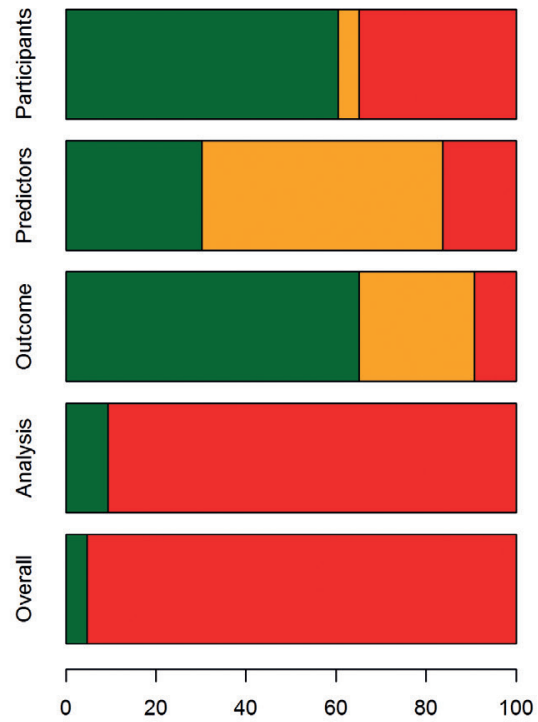

Comparing prediction models to the RCRI
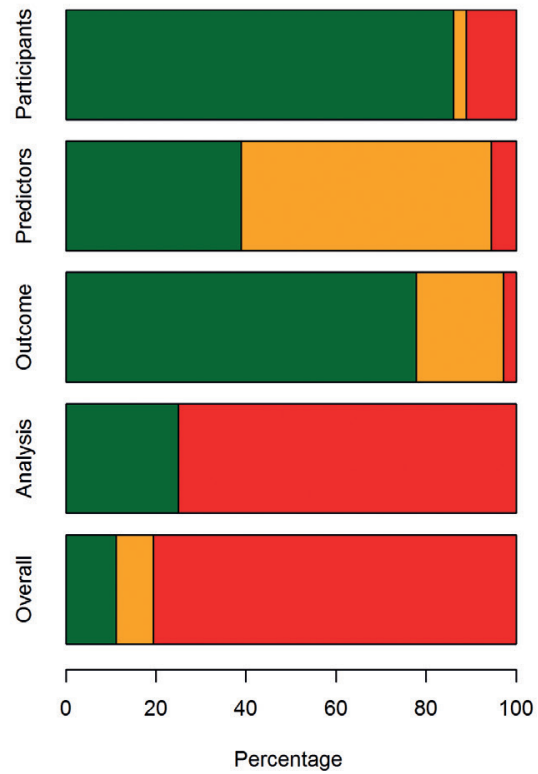

* Green refers to 'low' risk of bias; Orange is 'unclear' risk of bias and red represents 'high' risk of bias. 
Figure 4. Concern for applicability based on the PROBAST-tool

All included articles
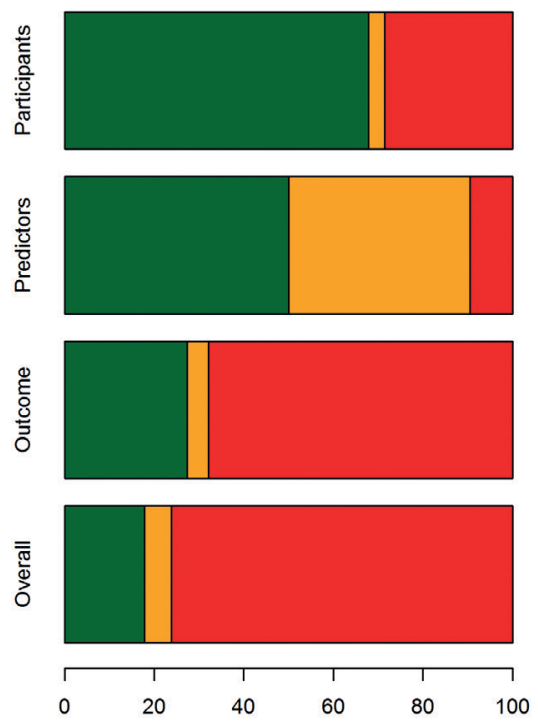

Comparing biomarkers to the RCRI
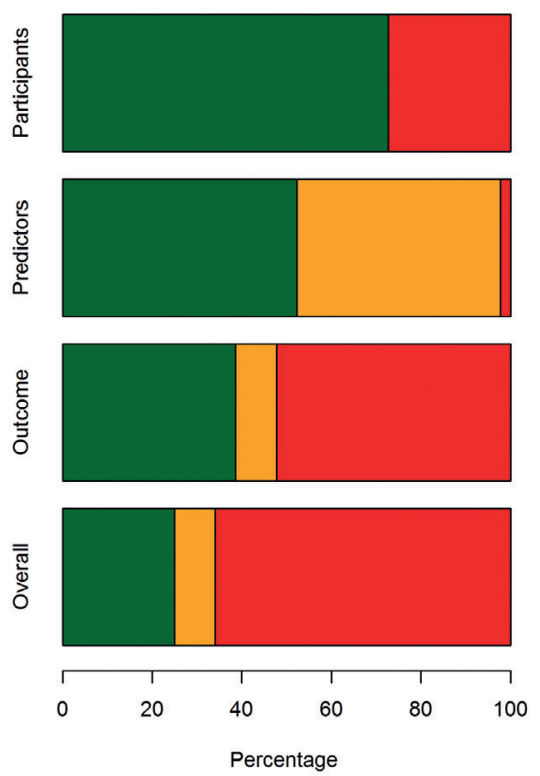

Added value to the RCRI
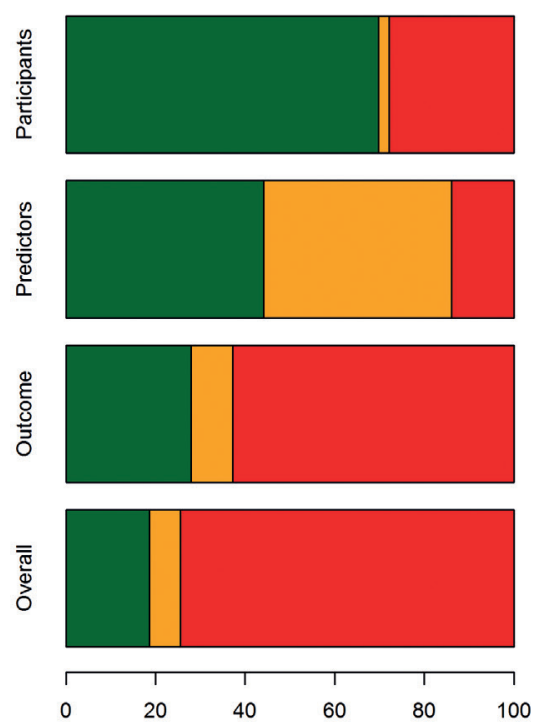

Comparing prediction models to the RCRI
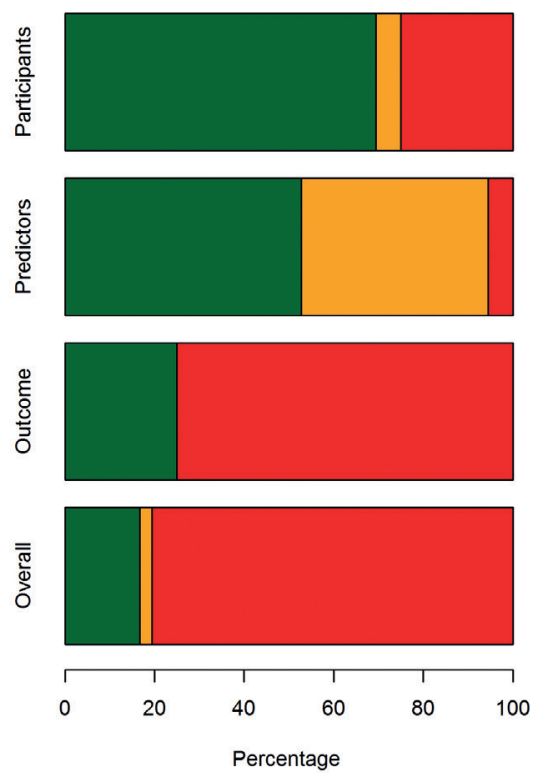

* Green refers to 'low' risk of bias; Orange is 'unclear' risk of bias and red represents 'high' risk of bias. 


\section{Predictive performance measures reported}

All included articles reported at least one performance measure (Table 5). For studies on the added value of (bio)markers to the RCRI, discrimination was reported in 39 (91\%) articles for which the majority of articles presented a c-statistic ( $n=32,74 \%)$. Compared to all included studies, discrimination measures were reported less often for studies on the added value of biomarkers to the RCRI (91\% and 74\%, respectively). Calibration was presented in only 8 (19\%) articles by means of an observed/expected ratio ( $n=6,14 \%)$, calibration plot $(n=2,5 \%)$ or a Hosmer Lemeshow test $(n=1$, $2 \%)$. Again, these performance measures were less frequently reported in articles evaluating the added value of predictors to the RCRI compared to all included articles (19\% vs 37\%, respectively). In total, 28 articles (33\%) reported both discrimination and calibration measures, of which 6 (15\%) investigated the added value of predictors to the RCRI. However, reclassification measures, presented as integrated discrimination improvement (IDI) or net reclassification improvement (NRI), were more often reported in articles investigating the added value of biomarkers to the RCRI compared to all included papers, as expected (35\% vs. 7\%, respectively).

\section{(Bio)markers added}

In Table 6, an overview of the (bio)markers added to the RCRI is provided sorted by the number of studies reporting on a particular (bio)marker. We identified 56 different added predictors of which 14 (25\%) were derived from blood, 19 (34\%) from imaging and 22 (39\%) were patient characteristics, such as smoking or age. In most studies, one predictor was added ( $n=41,73 \%)$ to the RCRI to improve risk prediction followed by two $(n=12,21 \%)$ and three predictors ( $n=3,5$ $\%)$ in the same model.

For the (bio)markers that have been added to the RCRI in at least three different studies, study characteristics and findings are described in further detail below. These biomarkers are $\mathrm{N}$-terminal pro-B type natriuretic peptide (NT-proBNP), brain natriuretic peptide (BNP), troponin and copeptin. 
Table 5. Performance measures reported for the extended model (i.e. articles reporting on the added value) or the compared model to the RCRI

\begin{tabular}{lcccc}
\hline & $\begin{array}{c}\text { All included } \\
\text { studies }\end{array}$ & $\begin{array}{c}\text { Added value } \\
\text { to the RCRI }\end{array}$ & $\begin{array}{c}\text { Comparison of } \\
\text { biomarkers }\end{array}$ & $\begin{array}{c}\text { Comparison } \\
\text { of prediction } \\
\text { models }\end{array}$ \\
\hline $\mathrm{n}$ & 84 & 43 & 44 & 36 \\
Performance category (\%) & & & & \\
$\quad$ Discrimination & $79(94.0)$ & $40(93.0)$ & $42(95.5)$ & $34(94.4)$ \\
Calibration & $31(36.9)$ & $8(18.6)$ & $15(34.1)$ & $19(52.8)$ \\
Reclassification & $17(20.2)$ & $15(34.9)$ & $3(6.8)$ & $3(8.3)$ \\
C-statistics (\%) & $76(90.5)$ & $32(74.4)$ & $38(86.4)$ & $33(91.7)$ \\
O/E (\%) & $20(23.8)$ & $6(14.0)$ & $11(25.0)$ & $7(19.4)$ \\
Calibration plot (\%) & $10(11.9)$ & $2(4.7)$ & $1(2.3)$ & $8(22.2)$ \\
Hosmer Lemeshow test (\%) & $4(4.8)$ & $1(2.3)$ & $2(4.5)$ & $4(11.1)$ \\
IDI (\%) & $6(7.1)$ & $6(14.0)$ & $1(2.3)$ & $0(0.0)$ \\
NRI (\%) & $16(19.0)$ & $14(32.6)$ & $2(4.5)$ & $3(8.3)$ \\
Other reported measures (\%) & & & & \\
Sensitivity & $33(39.3)$ & $5(11.6)$ & $21(47.7)$ & $10(27.8)$ \\
Specificity & $32(38.1)$ & $5(11.6)$ & $21(47.7)$ & $9(25.0)$ \\
Negative predictive value & $17(20.2)$ & $3(7.0)$ & $10(22.7)$ & $5(13.9)$ \\
Positive predictive value & $16(19.0)$ & $3(7.0)$ & $9(20.5)$ & $5(13.9)$ \\
Accuracy & $1(1.2)$ & $0(0.0)$ & $1(2.3)$ & $0(0.0)$ \\
\hline
\end{tabular}

O/E: observed/expected ratio; IDI: integrated discrimination improvement; NRI: net reclassification improvement ${ }^{\star *}$ Discrimination includes the following performance measures: c-statistics/AUC, sensitivity, specificity, negative predictive value, positive predictive value, positive predictive value and accuracy. Calibration includes O:E ratio, calibration plot and Hosmer Lemeshow test. Reclassification includes IDI and NRI. 
Table 6. Biomarkers/predictors added to the RCRI

\begin{tabular}{|c|c|c|}
\hline Predictor & $\begin{array}{l}\text { Number } \\
\text { of studies }\end{array}$ & Derivation \\
\hline NT-proBNP & 8 & Blood \\
\hline BNP & 5 & Blood \\
\hline Troponin & 5 & Blood \\
\hline Copeptin & 3 & Blood \\
\hline CRP & 2 & Blood \\
\hline fQRS of an ECG & 2 & Imaging \\
\hline NT-proBNP + CRP & 2 & Blood \\
\hline NT-proBNP + troponin & 2 & Blood \\
\hline Abdominal aortic aneurysm size & 1 & Other \\
\hline Age $>70$ years & 1 & Other \\
\hline Age + abdominal aortic aneurysm size & 1 & Other \\
\hline Age & 1 & Other \\
\hline Age + sex + copeptin & 1 & Other \\
\hline Anaerobic threshold & 1 & Other \\
\hline Anemia & 1 & Blood \\
\hline Angina pectoris & 1 & Other \\
\hline ASA classification & 1 & Other \\
\hline$A S A+S O R T+N S Q I P$ & 1 & Other \\
\hline Coronary artery calcium score (CACS) & 1 & Imaging \\
\hline Copeptin + NT-proBNP & 1 & Blood \\
\hline Coronary CT angiography & 1 & Imaging \\
\hline Duke Activity Status Index & 1 & Other \\
\hline ECG abnormalities & 1 & Imaging \\
\hline Left bundle branch block on a ECG & 1 & Imaging \\
\hline Rigth bundle branch block on a ECG & 1 & Imaging \\
\hline Echocardiography & 1 & Imaging \\
\hline Echocardiography + betablockers & 1 & Imaging \\
\hline EE ratio of echocardiography & 1 & Imaging \\
\hline Frailty & 1 & Other \\
\hline Jeopardy score & 1 & Imaging \\
\hline Left ventricular ejection fraction & 1 & Imaging \\
\hline Sex & 1 & Other \\
\hline
\end{tabular}


Table 6. Biomarkers/predictors added to the RCRI

\begin{tabular}{|c|c|c|}
\hline Predictor & $\begin{array}{l}\text { Number } \\
\text { of studies }\end{array}$ & Derivation \\
\hline Metabolic equivalent (METS) & 1 & Other \\
\hline METS + stress test & 1 & Other \\
\hline Multi vessel disease & 1 & Imaging \\
\hline Multi vessel diseause + CACS & 1 & Imaging \\
\hline Peak oxygen & 1 & Other \\
\hline Reactive hyperaemia peripheral arterial tonometry & 1 & Other \\
\hline Segment involvement + Jeopardy score & 1 & Imaging \\
\hline Segment involvement score & 1 & Imaging \\
\hline 6 minute walking test & 1 & Other \\
\hline Smoking & 1 & Other \\
\hline ST2 cardiac biomarker & 1 & Blood \\
\hline ST2 + troponin & 1 & Blood \\
\hline Survivin & 1 & Blood \\
\hline Thalium scan & 1 & Imaging \\
\hline Type of surgery & 1 & Other \\
\hline Type of surgery + age & 1 & Other \\
\hline Type of surgery + age + hypertension & 1 & Other \\
\hline Valve sclerosis & 1 & Imaging \\
\hline Valve stenosis & 1 & Imaging \\
\hline V-POSSUM & 1 & Other \\
\hline V-POSSUM + NT-proBNP & 1 & Blood \\
\hline V-POSSUM + troponin & 1 & Blood \\
\hline Wall abnormalities on an echocardiography & 1 & Imaging \\
\hline Total joint arthroplasty risk score & 1 & Other \\
\hline
\end{tabular}

* NT-prBNP: N-terminal prohormone of brain natriuretic peptide; BNP: Brain natriuretic peptide; CRP: C-reactive protein; fQRS of an ECG: fragmented QRS of an electrocardiogram (ECG); ASA: American Society of Anesthesiologists; SORT: Surgical Outcome Risk Tool; NSQIP: National Surgical Quality Improvement Program score; CT: computed tomography; E/e' ratio: ratio between early mitral inflow velocity and mitral annular early diastolic velocity; V-POSSUM: Vascular Physiologic and Operative Severity Score for the enUmeration of Mortality and Morbidity 
NT-proBNP

$\mathrm{N}$-terminal pro-B type natriuretic peptide (NT-proBNP) is generated by cardiomyocytes in the context of numerous triggers, most notably myocardial stretch. NT-proBNP has been increasingly used as a marker to establish the presence and severity of heart failure in both chronic ambulatory or acute decompensated heart failure setting. (67) We included eight articles reporting on the added predictive value of NT-proBNP to the RCRI in twelve different analyses. Two articles (10, 68) showed predictions of added value for multiple outcomes. Patients underwent either mixed non-cardiac $(n=4)$ or vascular surgery $(n=4)$. NT-proBNP was added to the RCRI on a continuous scale in 5 articles, on a dichotomous scale using a predefined threshold in 3 articles and on a categorical scale in 1 article. Figure 5 represents the added predictive value of NT-proBNP to the RCRI by means of the c-statistics to predict MACE, myocardial infarction, all-cause mortality, cardiovascular mortality or pulmonary edema. The majority of predictions were performed for the 30 day events $(n=8)$. Addition of NT-proBNP seems to improve the prediction of the RCRI model (i.e. delta c-statistic ranged from 0.04 to 0.16 ). The composition of predicted outcomes was very heterogeneous, especially for MACE. Also the number of included patients and outcome events was in the majority of the studies relatively low, i.e. median 455; range 122 to 2054.

\section{Brain Natriuretic Peptide (BNP)}

Similar to NT-proBNP, BNP is released by cardiomyocytes in case of myocardial stretch. BNP is used in clinical practice as a marker to establish the presence and severity of both chronic ambulatory or acute decompensated heart failure. (67) BNP was added to the RCRI in six analyses over five articles with one article describing two analyses using different outcomes and prediction horizons (i.e. in-hospital MACE and 1 year all-cause mortality). (15) Included articles reported most frequently on patients undergoing vascular surgery $(n=3)$. The outcome of interest in these articles was MACE ( $n=3)$, all-cause mortality $(n=1)$, a combination of both $(n=1)$ or troponin elevation $(n=1)$. Prediction horizons ranged from in-hospital to one year events. As none of the articles reported the c-statistics of the extended model (i.e. BNP added to the RCRI), we could not provide a forest plot. 
Figure 5. Forest plot of $\mathrm{c}$-statistics for the added value of NT-proBNP to the RCRI

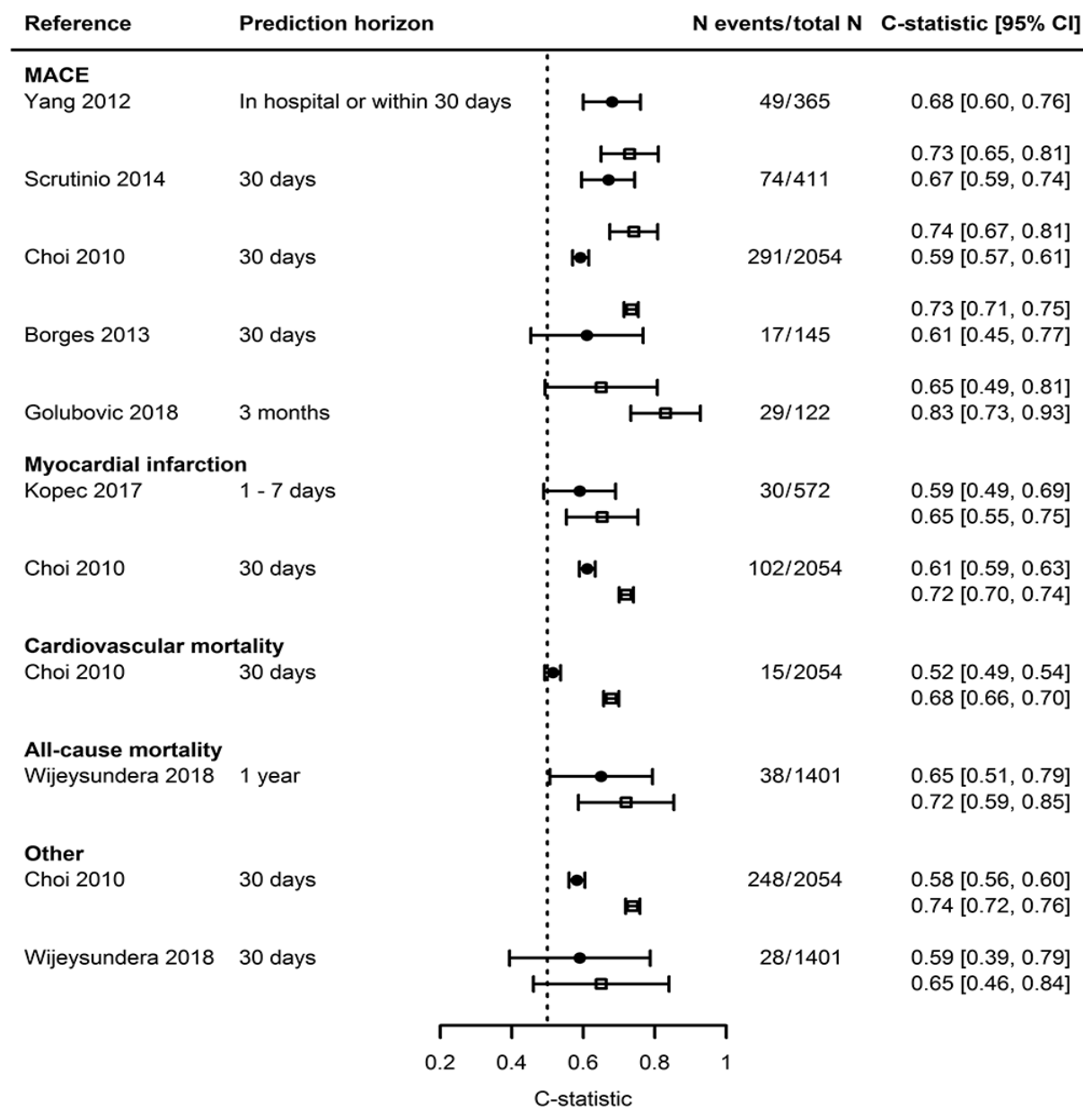

* Per article, two c-statistics with confidence intervals are presented. The upper (filled circle) represents the RCRI alone and the lower (open square) represents RCRI + NT-proBNP.

Troponin

Troponin is a protein that is involved in the contraction of cardiac muscle and is released by injured cardiomyocytes. Release of troponin may be due to myocardial cell death caused by ischemia, but also by e.g. normal turnover of myocardial cells, apoptosis or increased permeability of the cell wall. $(69,70)$ We included five articles reporting on the added predictive value of troponin to the RCRI in six analyses, of which one article (41) did the analyses in two populations. Included population concerned patients undergoing vascular $(n=3)$ or mixed non-cardiac surgery $(n=2)$. Troponin was added on a continuous scale or dichotomous scale in two and three studies, respectively. Included studies aimed to predict 30-day MACE ( $n=2)$, 
long term MACE ( $n=1), 30$ day MACE or all-cause mortality $(n=1)$ or myocardial infarction within three days of surgery $(n=1)$. Addition of troponin might improve the prediction of the RCRI model (i.e. delta c-statistic 0.06 to 0.11, Figure 6). However the extracted confidence intervals were wide, the studied patient populations (i.e. vascular and non-cardiac) were heterogeneous and the number of studies investigating the added value of troponin was low.

Figure 6. Forest plot of c-statistics for the added value of troponin to the RCRI

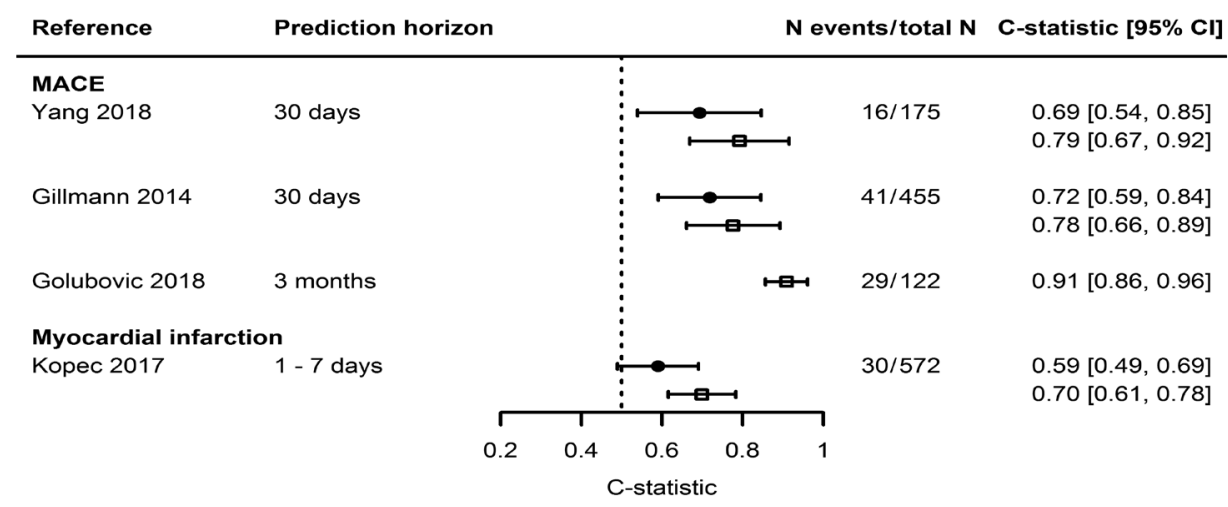

${ }^{*}$ Per article, two c-statistics with confidence intervals are presented. The upper (filled circle) represents the RCRI alone and the lower (open square) represents RCRI + troponin.

\section{Copeptin}

Copeptin is a novel marker of vasopressin activity, an antidiuretic hypothalamo-pituitary hormone, mainly regulated by changes in plasma osmolality, blood volume, and blood pressure. (71) Copeptin was added to the RCRI in three articles of which two studies reported on either the prediction of 30 day and long term MACE in the vascular surgical population. $(72,73)$ The other study (71) investigated the added value of copeptin to the RCRI to predict troponin elevation within two days after surgery in non-cardiac surgical patients. There was not sufficient information to summarize these studies in a forest plot. The c-statistics for the RCRI alone and the extended model was reported in one article, i.e. 0.714 and 0.752 , respectively. (72)

\section{Objective 2: comparison of the predictive value of single (bio)markers to the RCRI alone}

\section{Study design and study population}

In total, 44 studies compared the predictive performance of (bio)markers to the RCRI (Table 3). Similar to studies reporting on the added value of (bio)markers to the RCRI, most studies were cohort studies ( $n=40,91 \%$ ) and data were collected prospectively in 29 (66\%) studies. In 16 (37\%) and $12(28 \%)$ studies, patients originated from Europe and North America, respectively. Most 
included patients underwent non-cardiac surgery $(n=20,47 \%)$ followed by vascular surgery $(n=18$, 42\%). The surgical specialty was not specified in one study. Number of included participants was smaller for studies comparing of the predictive performance of (bio)markers to the RCRI relative to all included studies (median; IQR 255 [186, 604] and 528 [205, 1545], respectively). The number of events was not reported in one study. Most frequently used prediction horizons were during hospital admission $(n=7,16 \%)$, within the first 7 days $(n=6,14 \%)$ or 30 days $(n=23,53 \%)$ after surgery. In half of the included studies for this objective, MACE was the outcome to be predicted followed by all-cause mortality ( $n=7,16 \%)$ or a combination of both ( $n=7,16 \%)$. Three articles (7\%) reported predictions of other outcomes than MACE or all-cause mortality of which one study reported predictions for 21 different (non-cardiac) outcomes. (30) Similar to the articles included in the first objective, the number of published articles on the comparison of the predictive accuracy of (bio)markers to the RCRI increased over time with a peak in the 2012-2014 period (Figure 2).

\section{Composition of MACE}

For the 22 articles that used MACE as the outcome to be prediction, we found 21 different definitions i.e. compositions of MACE for studies that compared the prognostic ability of (bio) markers to the RCRI alone (Table 4). This heterogeneous composition resulted in 19 different used composites ranging from intraoperative hemodynamic adversity to cardiac death. Myocardial infarction was the most frequently used composite of MACE ( $n=20,95 \%)$.

\section{Risk of bias and concern for applicability}

Risk of bias and concern for applicability was scored and judged in a similar way for all included articles. Overall high risk of bias was rated in 42 (95\%) studies that compared the predictive performance of (bio)markers to the RCRI. Compared to studies included in the other objectives, risk of bias for participants was rated more frequently as high ( $n=20,45 \%)$. Most studies scored unclear risk of bias for predictors ( $n=26,59 \%$ ) due to no information on the definitions of the individual RCRI items. For the domain 'outcome' and for the domain 'analyses', ( $n=7,9 \%)$ and ( $n=41,93 \%)$ studies scored high risk of bias, respectively. Concern for applicability in at least one of the domains was scored high in 29 (65\%) of the included articles. This was mainly because of high concern for applicability in the domain 'outcome' ( $n=23,52 \%)$ due to inappropriate outcome used to be predicted (Figure 3 and 4).

As we did not observe differences in reasons for judgement of high or unclear risk of bias and concern for applicability among the different objectives, more detailed information on this topic is described below the subheading Risk of bias and concern for applicability as part of the first objective. 


\section{Predictive performance measures reported}

For studies comparing the prognostic ability of (bio)markers to the RCRI, predictive performance measures on discrimination, calibration and reclassification were reported in 96\%, 34\% and 7\%, respectively (Table 5). The c-statistics was presented in $86 \%$ of the included papers. Almost half of the articles that compared the predictive ability of (bio)markers to the RCRI reported sensitivity and specificity, which was more common compared to all included studies (48\% and 39\% for both, respectively). Besides sensitivity and specificity, the negative and positive predictive value were also more often used compared to studies on the added value of (bio)markers to the RCRI (23\% and $21 \%$ vs. $7 \%$ and $7 \%$, respectively). Calibration was presented as an observed/expected ratio (25\%), calibration plot (2\%) or a Hosmer Lemeshow test (5\%).

\section{Single (bio)markers compared}

In total, 141 analyses on the comparison of the predictive value of (bio)markers to the RCRI were identified in 44 included articles. An overview of (bio)markers for which the predictive performance was compared to the RCRI is presented in Table 7. We identified 47 unique predictors derived from blood ( $n=15,34 \%)$, imaging ( $n=15,34 \%$ ) or patient characteristics ( $n=17,39 \%$; e.g. age or metabolic equivalent (METS)).

For (bio)markers for which the predictive performance was compared to the RCRI in at least three different studies, the study characteristics are described in further detail below. These predictors were American Society of Anesthesiologist classification (ASA), BNP, NT-proBNP, troponin and C-reactive protein (CRP). 
Table 7. Biomarkers/predictors for which the predictive performance was compared to the RCRI

\begin{tabular}{|c|c|c|}
\hline Predictor & $\begin{array}{c}\text { Number of } \\
\text { studies }\end{array}$ & Derivation \\
\hline ASA classification & 12 & Other \\
\hline BNP & 10 & Blood \\
\hline NT-proBNP & 8 & Blood \\
\hline Troponin & 5 & Blood \\
\hline C-reactive protein & 3 & Blood \\
\hline Abnormal left ventricular ejection fraction & 2 & Imaging \\
\hline EE ratio of echocardiography & 2 & Imaging \\
\hline Wall motion abnormalities & 2 & Imaging \\
\hline Metabolic equivalent (METS) & 2 & Other \\
\hline CD40 & 1 & Blood \\
\hline eGFR & 1 & Blood \\
\hline H-FABP & 1 & Blood \\
\hline HFABP + Survivin & 1 & Blood \\
\hline NTproBNP+ troponin & 1 & Blood \\
\hline Platelet factor $\mathrm{V}$ & 1 & Blood \\
\hline Platelet P-selectin & 1 & Blood \\
\hline SCD40L & 1 & Blood \\
\hline St2 & 1 & Blood \\
\hline Survivin & 1 & Blood \\
\hline Survivin + CRP & 1 & Blood \\
\hline Abnormal echocardiography & 1 & Imaging \\
\hline $\begin{array}{l}\text { Abnormal left ventricular ejection fraction + wall } \\
\text { abnormalities }\end{array}$ & 1 & Imaging \\
\hline Coronary artery calcium score (CACS) & 1 & Imaging \\
\hline Coronary CT angiography & 1 & Imaging \\
\hline Dobutamine Stress Echocardiography & 1 & Imaging \\
\hline Jeopardy score & 1 & Imaging \\
\hline Left atrial volume index & 1 & Imaging \\
\hline Segment involvement in echocardiography & 1 & Imaging \\
\hline Systolic dysfunction & 1 & Imaging \\
\hline Systolic dysfuntion and left hypertrophy & 1 & Imaging \\
\hline Age & 1 & Other \\
\hline
\end{tabular}


Table 7. Biomarkers/predictors for which the predictive performance was compared to the RCRI

\begin{tabular}{lcc}
\hline Predictor & $\begin{array}{c}\text { Number of } \\
\text { studies }\end{array}$ & Derivation \\
\hline Age + surgical complexity & 1 & Other \\
Anaerobic threshold & 1 & Other \\
Ankle arm index & 1 & Other \\
Ankle arm index $\leq 0.9$ & 1 & Other \\
Ankle arm index $\geq 1.2$ & 1 & Other \\
Funcional capacity & 1 & Other \\
High age + ischemic heart disease & 1 & Other \\
Peak VO2 & 1 & Other \\
Pedal pulses absent on ankle arm index & 1 & Other \\
Positive stress test & 1 & Other \\
Reactive hyperaemia peripheral arterial & & Other \\
tonometry & 1 & Other \\
\hline minute walking test & & 1
\end{tabular}

* ASA: American Society of Anesthesiologists; BNP: Brain natriuretic peptide; NT-proBNP: N-terminal prohormone of brain natriuretic peptide; CRP: C-reactive protein; E/e' ratio: ratio between early mitral inflow velocity and mitral annular early diastolic velocity; eGFR: estimated Glomerular filtration rate; H-FABP; hearttype fatty acid binding protein; CT scan: computed tomography scan

\section{ASA}

The American Society of Anesthesiologists (ASA) physical status is a tool commonly used to classify a patient's physical fitness before surgery. It describes five classes of physical status ranging from ASA1 (i.e. healthy, non-smoking patient) to ASA5 (patient is expected to die within 24 hours). ASA6 is sometimes used to describe a brain-death organ donor. The ASA classification is not a prediction model, but a subjective and rapid assessment tool mostly based on the experience of the anesthesiologist. (36) The predictive ability of ASA was compared to the RCRI in 50 analyses over 12 included articles. Patients underwent a variety of surgical procedures, i.e. neurosurgery ( $n=2)$, $\operatorname{vascular}(n=3)$, general $(n=1)$, orthopaedic $(n=1)$, mixed non-cardiac surgery $(n=4)$ or unspecified $(n=1)$. The prediction horizon was most commonly within 30 days $(n=7)$ followed by in-hospital events $(n=4)$. MACE was the outcome to be predicted in six articles over seven analyses. Rohrig et al. reported two analyses in different patient populations. (44) Other predicted outcomes were myocardial infarction $(n=2)$ and all-cause mortality ( $n=3$ articles, 7 analyses). Other non-cardiac events were predicted in five articles over 33 analyses (Table 8). Bronheim et al. compared ASA to the RCRI to predict 21 different outcomes, (30) and Press et al. predicted four different non-cardiac outcomes. (31) Figure 7 and Table 8 show the reported c-statistics of ASA and RCRI. Overall, predictions were similar for both ASA and RCRI among the studied outcomes. 
Figure 7. Forest plot of c-statistics for the comparison of the predictive performance of ASA classification to the RCRI

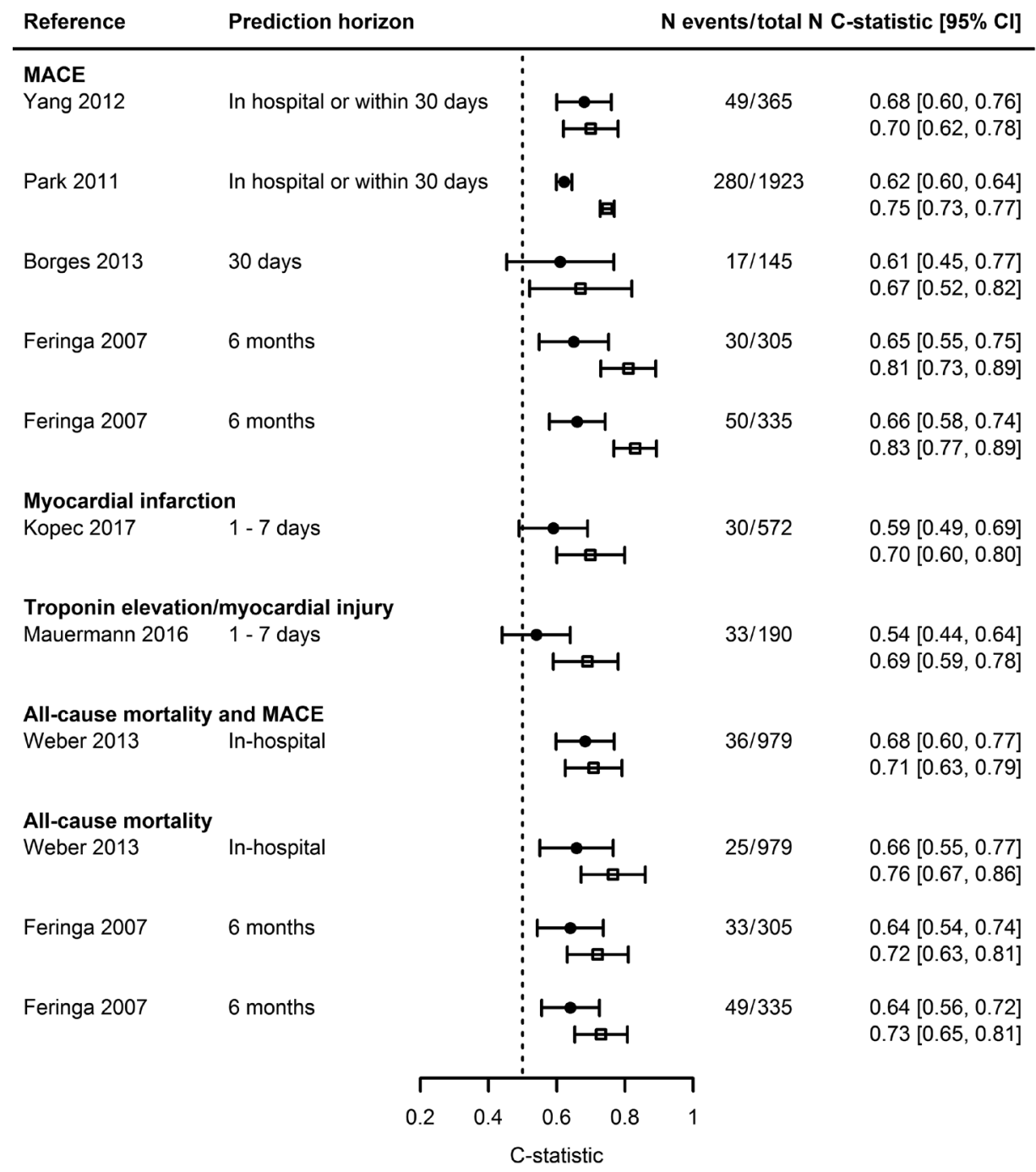

* Per article, two c-statistics with confidence intervals are presented. The upper (filled circle) represents the RCRI alone and the lower (open square) represents ASA. 


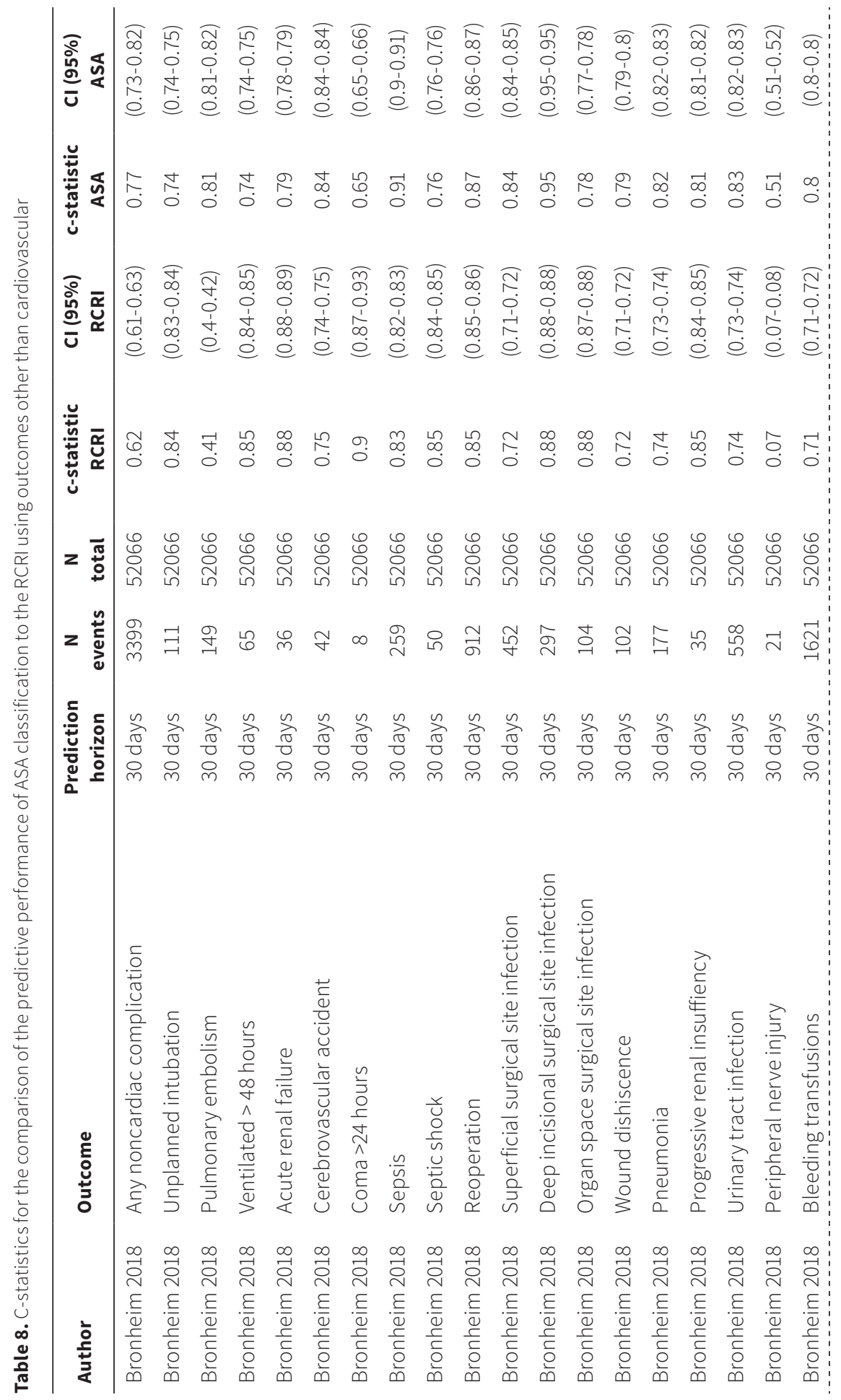


$B N P$

As mentioned before, BNP is released by cardiomyocytes due to myocardial stretch and used in clinical practice as a marker for heart failure. (67) We included 10 articles that compared the predictive ability of BNP to the RCRI over 14 different analyses. Rodseth et al. (74) reported predictions for BNP and RCRI alone using four different outcomes and Katsanos et al. (15) used two different outcomes (i.e. MACE and all-cause mortality, Figure 8). Predictions were made for seven different outcome categories, i.e. MACE ( $n=6)$, myocardial infarction ( $n=1)$, all-cause mortality $(n=2)$, a combination of the latter two $(n=1)$, cardiovascular mortality $(n=1)$, troponin elevation $(n=1)$ and other (non-cardiac) outcomes $(n=2)$. Overall, the $c$-statistic was on average 0.12 (range 0.02 to 0.24 ) higher as compared to the c-statistic of the RCRI alone. However, the number of included patients was low (i.e. less than 50 ) resulting in wide confidence intervals and no pooling of results could be performed due to the heterogeneous composition of MACE.

\section{NT-proBNP}

NT-proBNP is also used as marker for heart failure in clinical practice. (67) The predictive performance of NT-proBNP was compared to the RCRI alone in 12 analyses over eight included articles (Figure 9). Feringa et al. (42) reported four different analyses on two different outcomes (i.e. all-cause mortality and MACE) in two different patient populations. Weber et al. (17) reported prediction for two different outcomes (i.e. all-cause mortality and all-cause mortality and MACE). MACE was predicted in five different analyses, however the prediction horizon varied from inhospital to long term events in either vascular and non-cardiac surgical patients. Five articles studied NT-proBNP on a continuous scale, one on a categorical (75) and one on a dichotomous scale (9). For one article, the method of handling NT-proBNP was unclear. (42) Overall, the c-statistic was on average 0.10 (range 0.02 to 0.13 ) higher as compared to the c-statistic of the RCRI alone. However no inferences could be made due to the wide confidence intervals. 
Figure 8. Forest plot of c-statistics for the comparison of the predictive performance of BNP to the RCRI

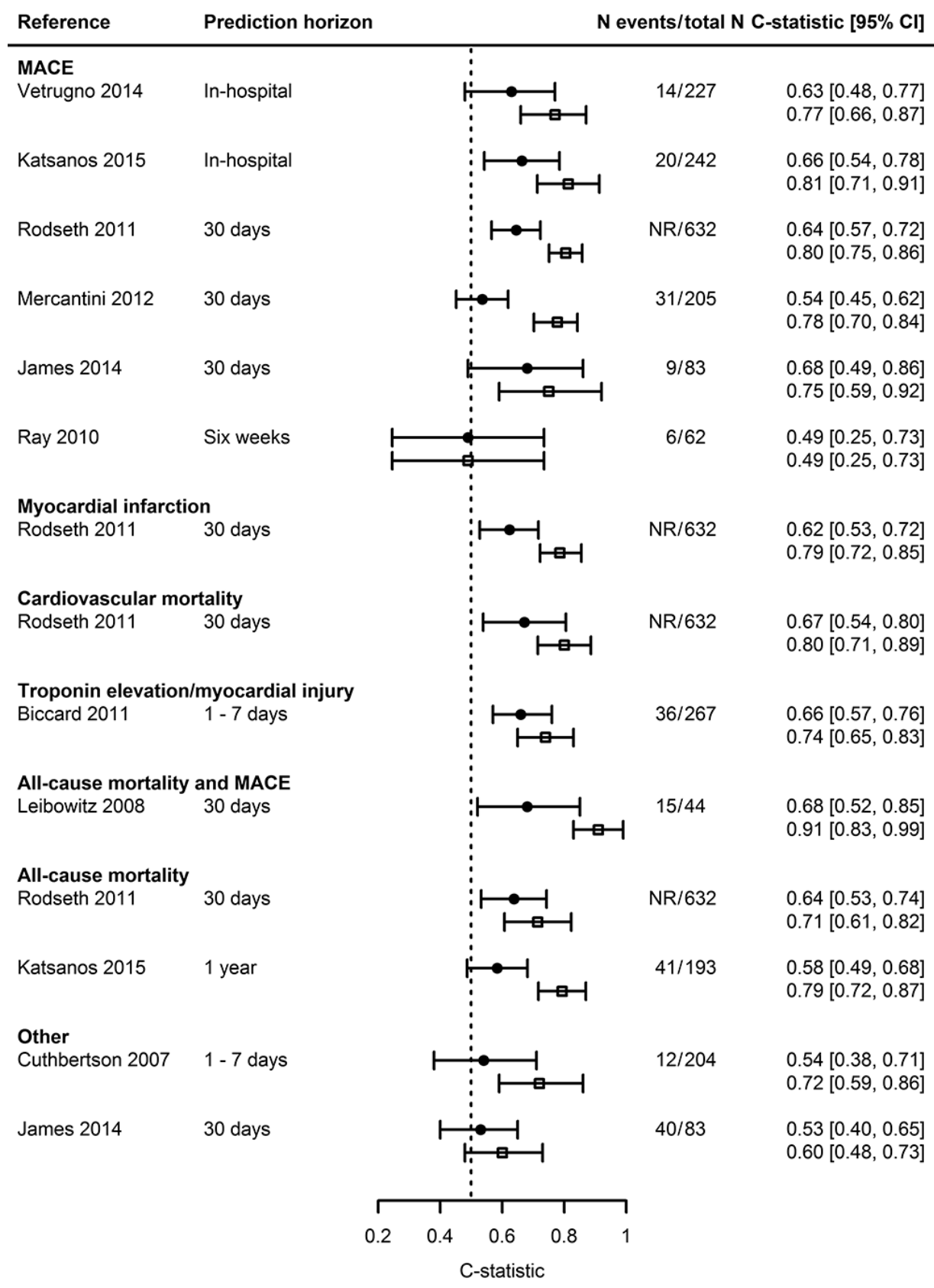

*Per article, two c-statistics with confidence intervals are presented. The upper (filled circle) represents the RCRI alone and the lower (open square) represents BNP alone 
Figure 9. Forest plot of c-statistics for the comparison of the predictive performance of NT-proBNP to the RCRI

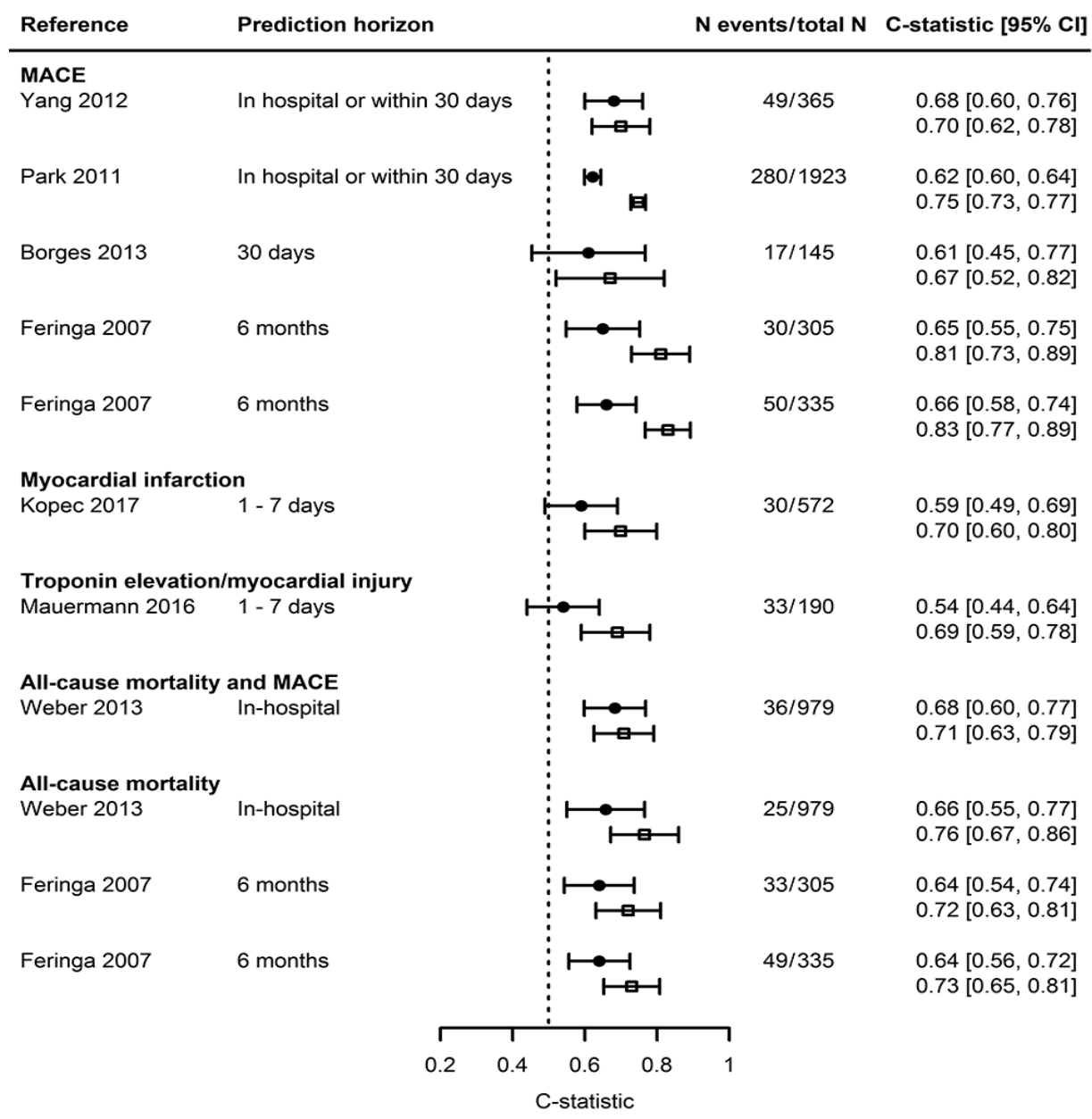

*Per article, two c-statistics with confidence intervals are presented. The upper (filled circle) represents the RCRI alone and the lower (open square) represents NT-proBNP alone.

\section{Troponin}

Troponin is a protein released by cardiomyocytes in case of myocardial ischemia. $(69,70)$. We included five articles reporting on seven analyses (Figure 10). Gualandro et al. (41) predicted MACE using troponin in two different populations (i.e. vascular and nonvascular patients). Although the aim for that study was to compare the predictive performance of troponin to the RCRI, only the c-statistics of troponin alone was reported. Included patients underwent either vascular ( $n=2)$ or non-cardiac surgery ( $n=3)$. Higher $\mathrm{c}$-statistics were observed for troponin alone compared to the 
RCRI (0.11, range 0.09 to 0.15 ), but no conclusion could be drawn as the number of events was low (i.e. below 60) and heterogeneous outcomes were used.

\section{C-reactive protein}

C-reactive protein (CRP) is a sensitive systemic marker of inflammation and tissue damage. The acute-phase response comprises the nonspecific physiological and biochemical responses of tissue damage, infection, inflammation, and malignant neoplasia. (76) Three articles compared the predictive ability of CRP to the RCRI (Figure 11). James et al. (77) made predictions for two different outcomes (i.e. MACE and postoperative complications). All included patients underwent non-cardiac surgery expect for patients included in the study of Ray et al.(78) who underwent orthopedic surgery. No difference in the predictive performance was found between the RCRI and CRP alone (mean delta c-statistic: 0.06 range - 0.12 to 0.22 ) as the number of patients over the included articles was low (i.e. $<100$ patients).

Figure 10. Forest plot of $\mathrm{c}$-statistics for the comparison of the predictive performance of troponin to the RCRI

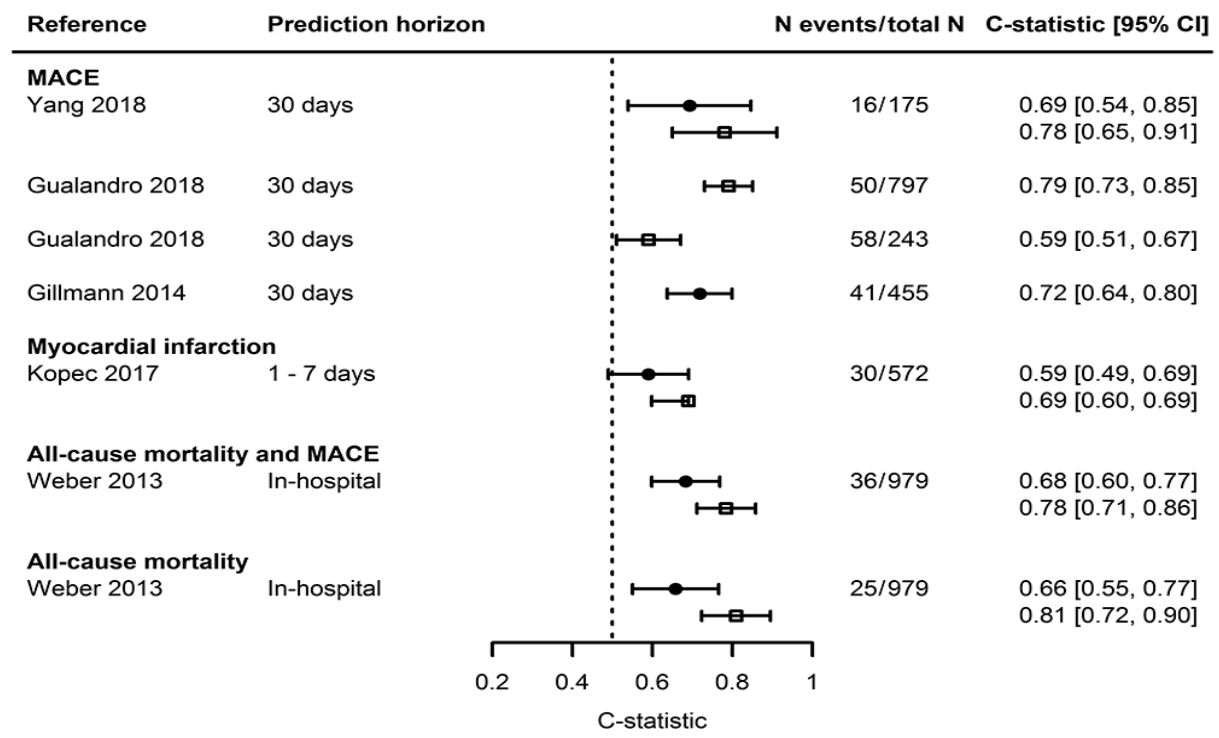

${ }^{*}$ Per article, two c-statistics with confidence intervals are presented. The upper (filled circle) represents the RCRI alone and the lower (open square) represents troponin alone. 
Figure 11. Forest plot of $\mathrm{c}$-statistics for the comparison of the predictive performance of CRP to the RCRI

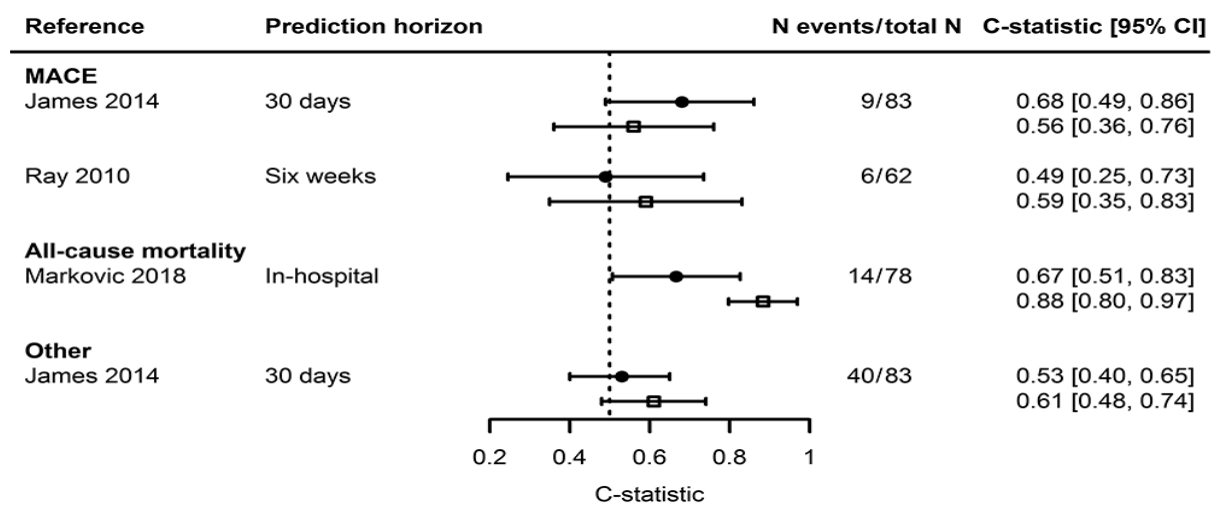

${ }^{*}$ Per article, two c-statistics with confidence intervals are presented. The upper (filled circle) represents the RCRI alone and the lower (open square) represents CRP alone.

\section{Objective 3: comparison of predictive value of prediction models to the RCRI alone}

\section{Study design and study population}

Thirty-six articles compared the predictive ability of the RCRI to another prediction model (Table 3). Most articles reported on cohort studies ( $n=34,94 \%)$. Retrospective data were more often used compared to studies on the added value of (bio)markers or studies that compared the predictive performance of (bio)markers to the RCRI (39\%, 28\% and 30\%, respectively). Included patients originated most commonly from Europe (48\%) or North America (31\%) and underwent most frequently non-cardiac (33\%) or vascular surgery (36\%). The median number of included patients was higher for this objective compared to studies reporting on the added value or the predictive performance of (bio)markers to the RCRI (median [IQR ]: 855 [265, 2609], 467 [199, 955] and 255 [186, 604], respectively). Similar to the articles included for the other two objectives, most frequently used prediction horizons were during hospital admission (28\%) or 30 days (53\%) after surgery. The outcome of interest was most often MACE (28\%) followed by all-cause mortality (17\%) and myocardial infarction or cardiac arrest (17\%). There was an increase in the number of publications over time, especially from 2009 onwards (Figure 2).

\section{Composition of MACE}

For included studies that used MACE as an outcome, all articles used a different definition meaning that the composition of MACE varied among the included articles (Table 4). We found 15 different composites for MACE. Similar to the articles reporting on the added value or the predictive performance of (biomarkers) to the RCRI, the most frequently used composite of MACE was myocardial infarction, i.e. in 12 out of 13 different definitions. The MACE definition also commonly included heart failure (69\%), cardiac arrest (54\%), cardiac death (39\%) or angina pectoris (31\%). 


\section{Risk of bias and concern for applicability}

Twenty-nine (81\%) studies that compared the predictive performance of the RCRI to other prediction models were judged as overall high risk of bias. Most studies scored low risk of bias for participants ( $n=29,81 \%)$. For predictors 20 (56\%) studies scored unclear risk of bias, for outcome 1 (3\%) and for analyses 27 (75\%) studies scored as high risk of bias. Reasons for these judgements were similar and thus described as part of the first objective. Comparable to studies included in the other objectives, most studies had high concern for applicability ( $n=29,81 \%$ ) (Figure 3 and 4). We observed no differences in reasons for judgement of high or unclear of risk of bias and concern for applicability among the different objectives. Accordingly, more detailed information is described below subheading Risk of bias and concern for applicability as part of the first objective.

\section{Performance measures reported}

Discrimination measures were reported in 34 (94\%) articles mostly using a c-statistics ( $n=33$, 92\%) (Table 5). Calibration was more often reported in articles that compared the predictive performance of other prediction models to the RCRI than articles that studies the added value or the comparison of the predictive ability of (bio)marker to the RCRI (53\%, 34\% and 33\%, respectively). This was in particularly by means of the calibration plot and observed/expected ratio. Reclassification measures were only reported in $3(8 \%)$ articles using a NRI.

\section{Prediction models compared}

We identified 65 analyses in 36 included papers that compared the predictive ability of the RCRI to other prediction models. An overview of prediction models for which the predictive performance was compared to the RCRI is presented in Table 9. We found 44 different prediction models of which 31 (70\%) prediction models were external validations of existing models, 9 (20\%) were new prediction models and 4 (9\%) updated the RCRI model.

For prediction models for which the predictive performance was compared to the RCRI in at least three different studies, the study characteristics are described in further detail below. These prediction models were ACS NSQIP score, Gupta MICA score, Goldman index and Detsky index.

\section{ACS NSQIP SCOre}

The American College of Surgeons National Surgical Quality Improvement Program (ACS NSQIP) surgical risk calculator is a decision-support tool based on multi-institutional clinical data, which can be used to estimate the risks of multiple outcomes (including myocardial infarction) of most operations. (79) We included six articles reporting nine different analyses (Figure 12). Copeland et al. (80) predicted MACE in two different populations (i.e. long versus low surgical duration) and Cohn et al. (19) reported predictions for three different outcomes (i.e. in-hospital and 30 day MACE and MICA). None of the articles showed preferences for one model over the other, except for Cohn et al. (19) for predicting myocardial infarction or cardiac arrest. However, there were only three events in this publication. 
Table 9. Prediction models for which the predictive performance was compared to the RCRI

\begin{tabular}{|c|c|}
\hline Predictor & Number of studies \\
\hline ACS NSQIP score & 6 \\
\hline Gupta MICA risk score & 4 \\
\hline Goldman index & 3 \\
\hline Detsky index & 3 \\
\hline CHADS2 score & 2 \\
\hline Glasgow Aneurysm Risk score & 2 \\
\hline Halm score & 2 \\
\hline POSSUM & 2 \\
\hline P-POSSUM & 2 \\
\hline R2CHADS2 score & 2 \\
\hline RCRI without diabetes melitus and low eGFR & 2 \\
\hline Thoracic RCRI & 2 \\
\hline Tu score & 2 \\
\hline VSGCRI & 2 \\
\hline ACE-27 + high age & 1 \\
\hline Adult comorbidity evaluation (ACE-27) score & 1 \\
\hline ANESCARDIOCAT & 1 \\
\hline$A S A+A C S N S Q I P$ & 1 \\
\hline ASA + frailty & 1 \\
\hline$A S A+S O R T$ & 1 \\
\hline Biochemistry and Haematology Outcome Model & 1 \\
\hline CHADS2VASC & 1 \\
\hline CR-POSSUM & 1 \\
\hline Eagle score & 1 \\
\hline Geriatric-Sensitive Perioperative Cardiac Risk Index & 1 \\
\hline Insulin + open surgery + high fibrinogen + CRP + NT-proBNP & 1 \\
\hline Long-Term Survival Score & 1 \\
\hline $\mathrm{MI}+\mathrm{Sex}+\mathrm{IDDM}+$ lowBMI + high age $+\mathrm{AF}$ & 1 \\
\hline modified Frailty Index & 1 \\
\hline
\end{tabular}


Table 9. Prediction models for which the predictive performance was compared to the RCRI

\section{Predictor}

Number of studies

new Model 1

new Model 2

1

new Model 3

1

Preoperative risk score of the estimation of physiological ability and surgical stress score

RCRI with redefined high risk surgery

RCRI with redefined high risk surgery and clinical characteristics

RCRI without IDDM and high creatinin

South African Vascular Surgical Cardiac Risk Index

1

Surgical Outcome Risk Tool

1

Surgical risk score

1

TJA individual factors

TJA risk score

1

Vascular biochemical and haematological outcome model 1

Vascular Study Group of New England Cardiac Risk Index + anemia 1

V-POSSUM

* ACS NSQIP: American College of Surgeons National Surgical Quality Improvement Program; MICA: myocardial infarction and cardiac arrest; CHADS2 score: congestive heart failure, hypertension, age $\geq 75$ years, diabetes mellitus, stroke (double weight); POSSUM: Physiologic and Operative Severity Score for the enUmeration of Mortality and Morbidity; P-POSSUM: Portsmouth-POSSUM; R2CHADS2: CHADS2 score added with renal failure (double weighted); VSG-CRI: Vascular Study Group of New England Cardiac Risk Index; ACE-27: Adult comorbidity evaluation-27; SORT: Surgical Outcome Risk Tool; CHADS2VASc: CHADS2 added with vascular disease, age 65-74 years and sex; CR-POSSUM: POSSUM score for colorectal surgical patients; New model 1: age, sex, history of coronary revascularization, aortic or mitral valve disease, arrhythmia, hypertension, carotid artery stenosis, hypovolemia, chronic renal failure, emergency surgery, neurosurgery, thoracic surgery, major vascular surgery, hematopoietic/lymphatic surgery, gastro-intestinal surgery; New model 2: age, ASA, neurosurgery, thoracic surgery, major vascular surgery, hematopoietic/lymphatic surgery, gastro-intestinal surgery; New model 3: history of myocardial infarction, age $>70$, IDDM, Female, BMI < 18, Operation time > 2.5 hours, AF, intraoperative hypotension; TJA: total joint arthroplasty; V-POSSUM: POSSUM for vascular surgical patients 
Figure 12. Forest plot of c-statistics for the comparison of the predictive performance of ACS NSQIP score to the RCRI

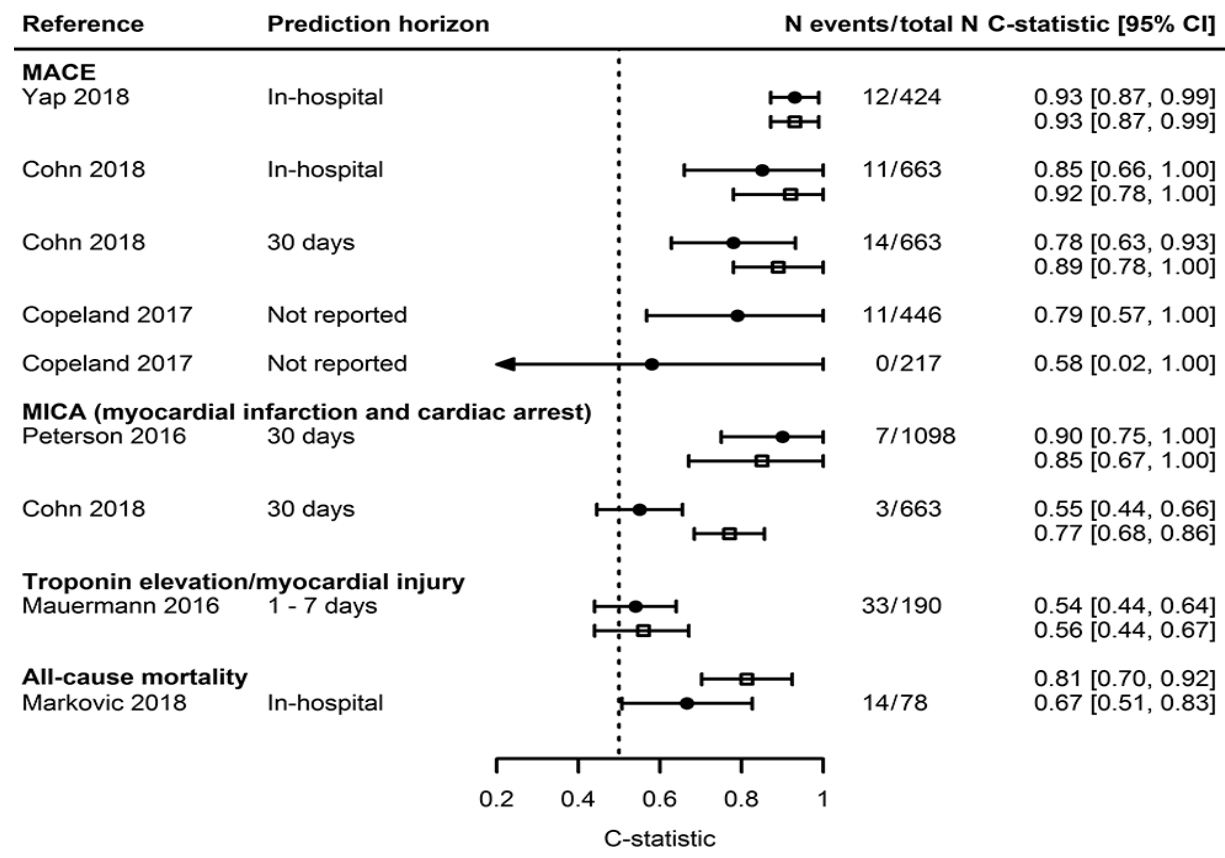

*Per article, two c-statistics with confidence intervals are presented. The upper (filled circle) represents the RCRI alone and the lower (open square) represents the NSQIP score.

\section{Gupta MICA score}

The Gupta MICA cardiac risk calculator was developed in 2011 and provides a risk estimate of 30-day myocardial infarction or cardiac arrest (MICA) in patients undergoing non-cardiac surgery. Data from the ACS NSQIP was used for development of the model. (21) Predictions for MACE were made in two analyses by Cohn et al. (19) (i.e. prediction horizon for in-hospital and 30 day events) and two analyses by Copeland et al. (80) (i.e. patients with long versus short surgical duration). No differences in predictions among the RCRI and Gupta model were found. Furthermore, three articles predicted 30-day MICA in six analyses, which resulted in better predictive performance of the Gupta MICA score compared to RCRI alone. Asuzu et al. (22) reported three analyses among patients undergoing open procedures, laparoscopic procedures or all included procedures and Alrezk et al. (81) studied geriatric and non-geriatric patients. Information regarding the c-statistics are presented in Figure 13.

\section{Goldman index}

The Goldman index represents a multivariable approach to estimate cardiac risk in patients undergoing non-cardiac procedures. (47) The model was developed in 1977 and can be considered 
as a previous version of the RCRI. The RCRI and Goldman index were validated in three articles over eight analyses (Figure 14). Press et al. (31) reported on predictions on five different outcomes (i.e. MACE and four non-cardiac outcomes) in patients undergoing vascular surgery. No difference in c-statistics was found, which could be explained by the fact that both models were not originally developed to predict non-cardiac outcomes. Katsanos et al. (15) compared the RCRI to the Goldman index to predict in-hospital MACE and 1 year all-cause mortality in patients undergoing orthopaedic surgery and Pantoja Muñoz et al. (82) used both models to predict in-hospital MACE. For the latter, only sensitivity and specificity measures were reported and therefore, data was not sufficient for showing results in a forest plot.

\section{Detsky index}

The Detsky index is a modified version of an index that was previously generated by Goldman in 1977. (48) This model was developed in 1986 and revised to the RCRI by Lee et al. in 1999. (6) We identified the same articles that compared the predictive performance of Detksy to the RCRI as for the comparison between Goldman and the RCRI (Figure 15). As results are similar, more detailed information can be found below Goldman index.

Figure 13. Forest plot of c-statistics for the comparison of the predictive performance of Gupta MICA risk score to the RCRI

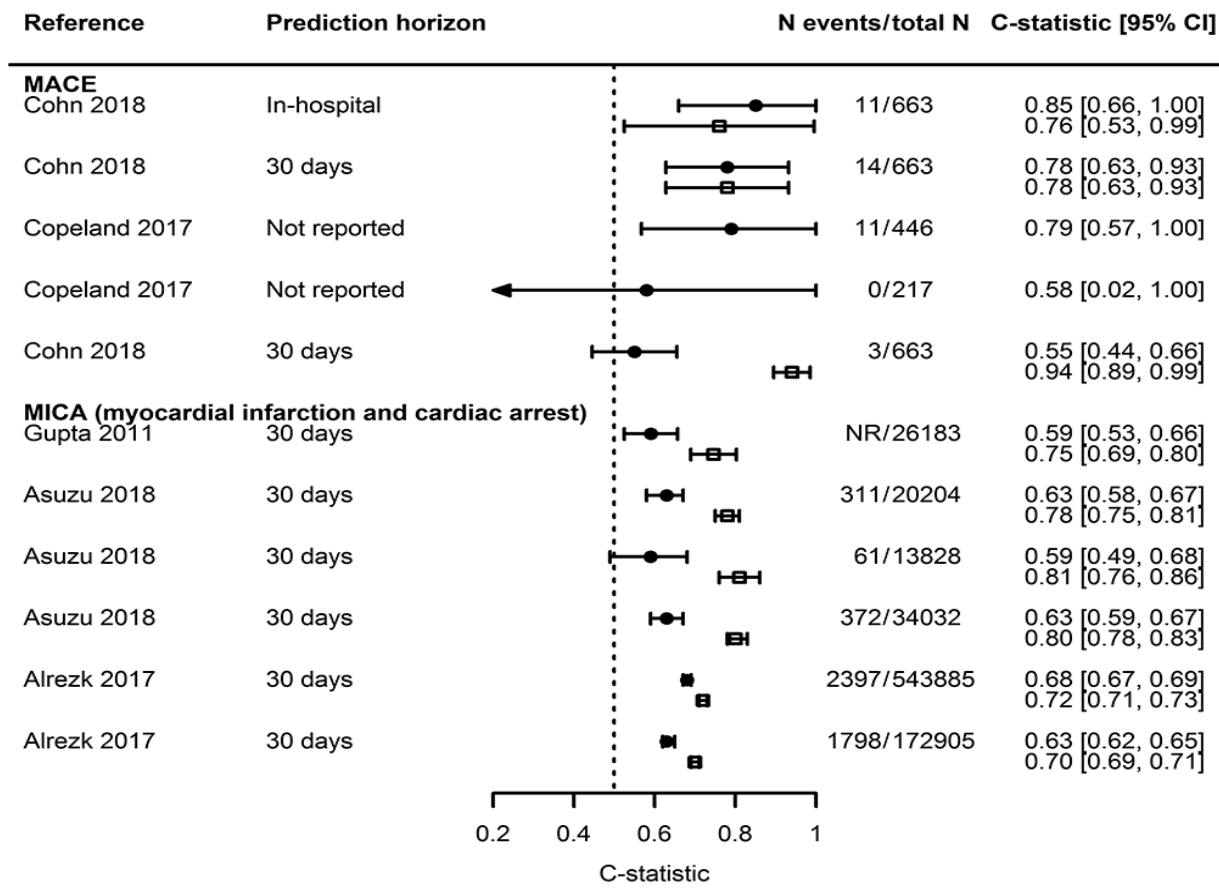

*Per article, two c-statistics with confidence intervals are presented. The upper (filled circle) represents the RCRI alone and the lower (open square) represents the Gupta MICA risk score. 
Figure 14. Forest plot of c-statistics for the comparison of the predictive performance of Goldman index to the RCRI

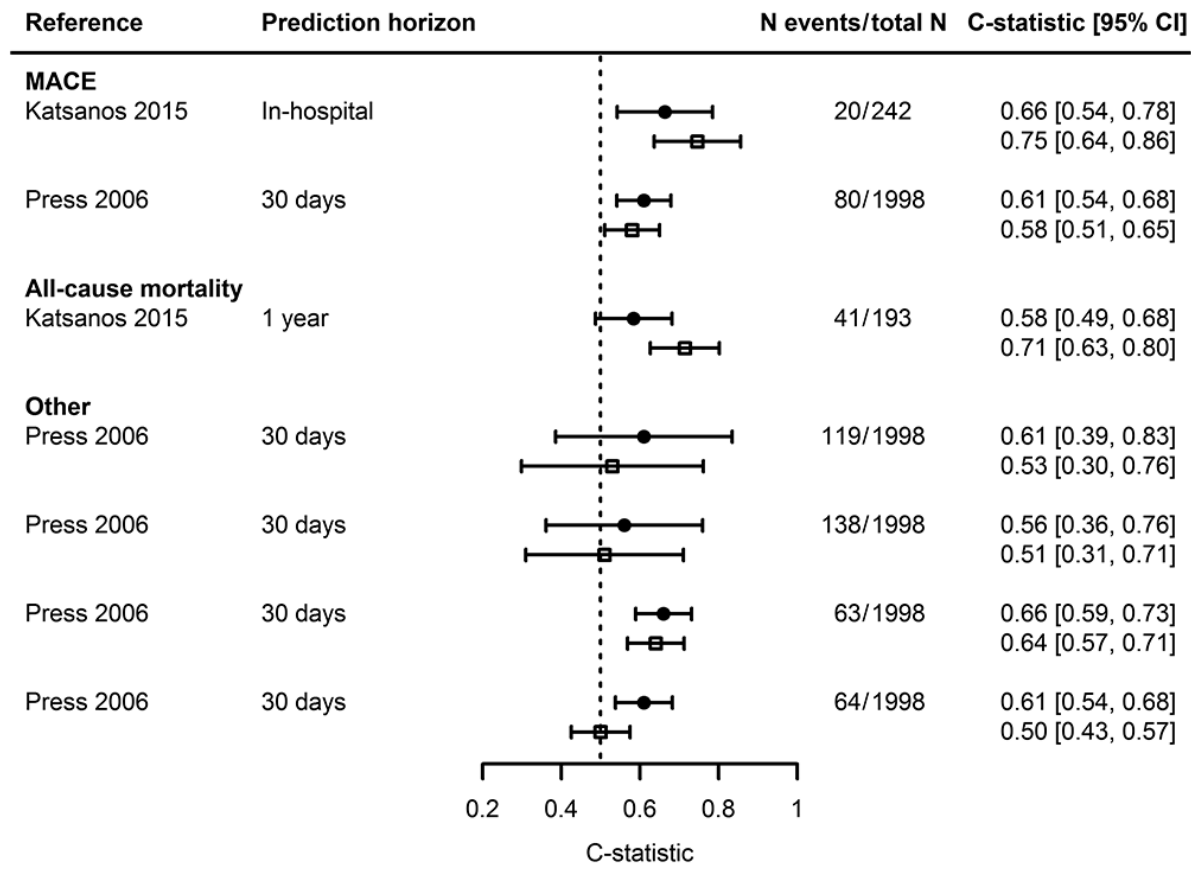

*Per article, two c-statistics with confidence intervals are presented. The upper (filled circle) represents the RCRI alone and the lower (open square) represents the Goldman index.

Figure 15. Forest plot of c-statistics for the comparison of the predictive performance of Detsky index to the RCRI

\begin{tabular}{|c|c|c|c|c|}
\hline Reference & Prediction horizon & & $\mathrm{N}$ events/total $\mathrm{N}$ & C-statistic $[95 \% \mathrm{CI}]$ \\
\hline $\begin{array}{l}\text { MACE } \\
\text { Katsanos } 2015\end{array}$ & In-hospital & $\vdots \longmapsto$ & $20 / 242$ & $\begin{array}{l}0.66[0.54,0.78] \\
0.78[0.67,0.88]\end{array}$ \\
\hline Press 2006 & 30 days & $\stackrel{\mapsto \mapsto-1}{\mapsto \bullet}$ & $80 / 1998$ & $\begin{array}{l}0.61[0.54,0.68] \\
0.66[0.59,0.73]\end{array}$ \\
\hline $\begin{array}{l}\text { All-cause mortality } \\
\text { Katsanos } 2015\end{array}$ & 1 year & $\stackrel{\leftrightarrow}{\longmapsto}$ & $41 / 193$ & $\begin{array}{l}0.58[0.49,0.68] \\
0.72[0.64,0.81]\end{array}$ \\
\hline $\begin{array}{l}\text { Other } \\
\text { Press } 2006\end{array}$ & 30 days & $\longrightarrow$ & $119 / 1998$ & $\begin{array}{l}0.61[0.39,0.83] \\
0.56[0.33,0.79]\end{array}$ \\
\hline Press 2006 & 30 days & $\longrightarrow$ & $138 / 1998$ & $\begin{array}{l}0.56[0.36,0.76] \\
0.53[0.33,0.73]\end{array}$ \\
\hline Press 2006 & 30 days & $\longmapsto$ & $63 / 1998$ & $\begin{array}{l}0.66[0.59,0.73] \\
0.68[0.61,0.75]\end{array}$ \\
\hline Press 2006 & 30 days & 引ִ & $64 / 1998$ & $\begin{array}{l}0.61[0.54,0.68] \\
0.56[0.49,0.63]\end{array}$ \\
\hline & 0.2 & $\begin{array}{ll}1 & 1 \\
0.4 & 0.6\end{array}$ & ᄀ & \\
\hline
\end{tabular}




\section{DISCUSSION}

\section{Summary of main results}

This systematic review included 84 articles of which 43 articles reported on the added value of predictors to the RCRI. We identified 56 unique predictors that were added to the RCRI to improve risk prediction, which were derived from blood (25\%), imaging (34\%), were patient characteristics (30\%). Addition of NT-proBNP or troponin seems to improve the prediction of the RCRI model (i.e. delta c-statistic ranged from 0.04 to 0.16 and 0.06 to 0.11 for NT-proBNP and troponin, respectively). For BNP and copeptin, no data on predictive performance (i.e. c-statistic) were available meaning that no reliance on the validity of these findings can be placed The composition of predicted outcomes was very heterogeneous, especially for MACE for which 40 different definitions were reported. Also the number of included patients and outcome events was in the majority of the studies relatively low. The predictive performance of (bio)markers alone was compared to the RCRI in 44. Forty-seven unique (bio)markers were identified that compared to the RCRI. Predictors were derived from blood (34\%), imaging (34\%) or patient characteristics (39\%). Predictions were similar for both ASA and the RCRI among the studied outcomes. For BNP, NT-proBNP and troponin, the c-statistic was on average $0.14,0.10$ and 0.11 , respectively, higher as compared to the c-statistic of the RCRI alone. No difference in the predictive performance was found between the RCRI and CRP alone. Again, we observed large heterogeneity among the included studies due to the low number of included patients resulting in wide confidence intervals and the varying composition of outcomes. For the third objective, in 36 articles we found 44 different prediction models that were compared to the RCRI. None of these prediction models showed preferences for one model over the RCRI.

\section{Certainty of the evidence}

There is currently no official GRADE guidance available for grading summarized results of prognostic models. Therefore, we use the recommendations of GRADE for assessment of evidence about prognosis by lorio et al. $(65,66)$. The following GRADE criteria were taken into consideration for the assessment of certainty of evidence; risk of bias, imprecision, inconsistency, indirectness, and publication bias.

Risk of bias was assessed using PROBAST $(50,51)$ and was rated as 'high' in at least one domain in 75 (89\%) of all included studies. Reasons for judgement of high risk of bias were mainly due to inappropriate in- or exclusion of participants, low number of events, not reporting of relevant performance measures (i.e. discrimination and/or calibration) at all or without uncertainty measures. In addition, none of the included articles used imputation for handling of missing data.

Indirectness refers to the applicability of the prediction model to the proposed patient population and whether the measured outcome corresponds to the intended outcome to be predicted. 
Concern for applicability was judged 'high' in 61 (73\%) of all included studies, mainly due to strict in- and exclusion criteria and the use of other outcomes than the outcome that was used in the development study, i.e. MACE. Many included articles reported predictions for, e.g. other cardiac complications, non-cardiac complications and all-cause mortality.

Inconsistency is defined as variability in point estimates, extent of overlap in confidence intervals, and considering where point estimates lay in relation to decision thresholds. Imprecision refers to precision of the risk estimate based on the 95\% confidence interval. For this review, metaanalyses of the predictive performance measures (including c-statistic) were not possible due to extreme clinical and methodological heterogeneity across studies. This heterogeneity included a wide variety in: (bio)markers and prediction models added or compared to the RCRI, outcome definitions, prediction horizons and no uniformity in scale on how the predictor or prediction model was added/compared to the RCRI (i.e. continuous, categorical or dichotomous). As a result, we were not able to pool the results meaning that we cannot judge certainty of evidence based on inconsistency and imprecision.

Currently, there is no established standard for assessing the likelihood of publication bias in research on prognostic models. In addition, publication bias could also not be assessed due to the low number of included papers reporting on a particular predictor. However, many studies in this research field have measured (bio)markers and collected the items of the RCRI and/or other prediction models, but did not publish the results (yet) on their predictive performance.

Due to the extreme heterogeneity in predictors investigated and outcomes used and poor reporting of predictive performance measures, pooling of results was deemed impossible. In addition, three-quarter of the included studies were judged with high risk of bias and high concern for applicability. Overall, the certainty of evidence was therefore judged as very low.

\section{Limitations of the review}

Several limitations should be addressed. Firstly, our search was performed in October 2018 meaning that we have missed one year of articles that could be eligible studies for this review. Secondly, we excluded articles for which the full text was not available (4\%). This may have led to an underestimation of the number of predictors adding or comparing its predictive performance to the RCRI. Thirdly, we encountered missing data for many of the included studies especially in the predictive performance measures. We have not contacted study authors for additional information (e.g. on performance measures) yet, but will be done before submission of the full review. 


\section{Applicability of findings to clinical practice and policy}

In almost half of the included articles, the outcome of interest was MACE. MACE however was poorly defined, as we found 40 different compositions of MACE. Accordingly, comparison of articles studying similar predictions was complex or even impossible. Jammer et al. (83) published a guideline to define patient centered outcome measures for large clinical trials in perioperative medicine. In this paper, MACE was defined as one or more of the following: non-fatal cardiac arrest, acute myocardial infarction, congestive heart failure, new cardiac arrhythmia and angina. However, guidance was only based on one publication (84) and they did not adopt a formal voting system or a Delphi consensus process. As a response to this, the 'Standardized Endpoints for Perioperative Medicine' (StEP) Group was established with the aim to come up with a consensus process of standardizing endpoints in perioperative medicine. (85) Several guidance papers came out of this initiative, (86-90), but no standardized definition on cardiovascular endpoints is published yet.

Besides the variety in predicted outcomes, we identified a large amount of different (bio)markers and prediction models added or compared to the RCRI to improve risk prediction. Most (bio) markers and prediction models that were added or compared its predictive performance to the $\mathrm{RCRI}$ were only studied once meaning that selecting promising predictors is currently not possible. Extra complexity in the comparison of different studies might arise when (bio)markers, especially derived from blood are studied on a different scale (i.e. continuous, categorical or dichotomous) using different thresholds. Imaging (bio)markers might be exposed to subjective interpretation of the assessor resulting in bias.

From the clinical perspective, usefulness of a prediction model depends not only on good predictive performance but also on health care resources. This includes costs, whether measurement is invasive or not, times to measure a certain (bio)marker and easiness to calculate predictions. If tests are for example very expensive and take a long time to be measured and interpreted, the model might not be suitable in clinical practice with limited time to make decisions. In addition, we found 44 articles that compared the predictive performance of single biomarkers to the RCRI. However, treatment decisions are normally based on information of multiple predictors and therefore, making predictions based on single (bio)markers is less relevant. (91)

\section{Agreements and disagreements with other studies or reviews}

To our knowledge, this is the first systematic review that provided a comprehensive overview of all (bio)markers and prediction models that have been added or compared its predictive performance to the RCRI to improve risk prediction in patients undergoing non-cardiac surgery. However, there is one individual patient data meta-analysis including data of six studies comparing 
the predictive performance of BNP to the RCRI in vascular surgical patients. (74) They found higher C-statistics for BNP compared to the RCRI (0.62; 95\% Cl: $0.55-0.69$ and 0.81; 95\% Cl: $0.75-0.86)$. However, authors assign this difference to the fact that the RCRI was derived from a population of predominantly non-cardiac and nonvascular surgery patients. Therefore, they recommend that further research should be undertaken to determine whether the RCRI improves pre-operative risk stratification in patients primarily risk stratified using BNP. (74)

\section{Author's conclusions}

\section{Implications for practice}

We did not find a substantial added value of any (bio)marker(s) to the RCRI to preoperatively predict postoperative adverse outcomes in patients undergoing non-cardiac surgery. No difference in predictive performance was observed for the comparison of the RCRI to a single (set of) biomarker. Also, comparative models in this field do not predict better than the RCRI.

\section{Implications for research}

As results of this systematic review are inconclusive, further research is needed on the added predictive value of (bio)markers to improve risk prediction using the RCRI. Preferably, resources in terms of costs, time to measure and measurement invasiveness of these (bio)markers should be taken into account. Consensus on the definition of MACE is mandatory to be able to compare studies and provide stronger evidence. Furthermore, individual patient data meta-analyses in this research area might be beneficial to identify (bio)markers with added value to the RCRI. 


\section{REFERENCES}

1. Rose J, Weiser TG, Hider P, Wilson L, Gruen RL, Bickler SW. Estimated need for surgery worldwide based on prevalence of diseases: a modelling strategy for the WHO Global Health Estimate. Lancet Glob Health. 2015;3 Suppl 2:S13-20.

2. Weiser TG, Haynes AB, Molina G, Lipsitz SR, Esquivel MM, Uribe-Leitz T, et al. Estimate of the global volume of surgery in 2012: an assessment supporting improved health outcomes. Lancet. 2015;385 Suppl 2:S11.

3. Global patient outcomes after elective surgery: prospective cohort study in 27 low-, middleand high-income countries. Br J Anaesth. 2016;117(5):601-9.

4. Fleisher LA, Fleischmann KE, Auerbach AD, Barnason SA, Beckman JA, Bozkurt B, et al. 2014 ACC/AHA guideline on perioperative cardiovascular evaluation and management of patients undergoing noncardiac surgery: a report of the American College of Cardiology/American Heart Association Task Force on Practice Guidelines. Circulation. 2014;130(24):e278-333.

5. Kristensen SD, Knuuti J, Saraste A, Anker S, Botker HE, De Hert S, et al. 2014 ESC/ESA Guidelines on non-cardiac surgery: cardiovascular assessment and management The Joint Task Force on non-cardiac surgery: cardiovascular assessment and management of the European Society of Cardiology (ESC) and the European Society of Anaesthesiology (ESA). European Heart Journal. 2014;35(35):2383-431.

6. Lee TH, Marcantonio ER, Mangione CM, Thomas EJ, Polanczyk CA, Cook EF, et al. Derivation and prospective validation of a simple index for prediction of cardiac risk of major noncardiac surgery. Circulation. 1999;100(10):1043-9.

7. Ford MK, Beattie WS, Wijeysundera DN. Systematic Review: Prediction of Perioperative Cardiac Complications and Mortality by the Revised Cardiac Risk Index. Annals of Internal Medicine. 2010;152(1):26-W7.

8. Gillmann H-J, Meinders A, Grosshennig A, Larmann J, Buente C, Calmer S, et al. Perioperative Levels and Changes of High-Sensitivity Troponin T Are Associated With Cardiovascular Events in Vascular Surgery Patients. Critical Care Medicine. 2014;42(6):1498-506.

9. Kopec M, Duma A, Helwani MA, Brown J, Brown F, Gage BF, et al. Improving Prediction of Postoperative Myocardial Infarction With High-Sensitivity Cardiac Troponin T and NTproBNP. Anesthesia and Analgesia. 2017;124(2):398-405.

10. Choi JH, Cho DK, Song YB, Hahn JY, Choi S, Gwon HC, et al. Preoperative NT-proBNP and CRP predict perioperative major cardiovascular events in non-cardiac surgery. Heart. 2010;96(1):56-62.

11. Scrutinio D, Guido G, Guida P, Passantino A, Angiletta D, Santoro D, et al. Combined Use of High-sensitivity C-Reactive Protein and N-Terminal Pro-B-type Natriuretic Peptide for Risk Stratification of Vascular Surgery Patients. Annals of Vascular Surgery. 2014;28(6):1522-9.

12. Noordzij PG, Boersma E, Bax JJ, Feringa HHH, Schreiner F, Schouten O, et al. Prognostic value of routine preoperative electrocardiography in patients undergoing noncardiac surgery. American Journal of Cardiology. 2006;97(7):1103-6.

13. van Klei WA, Bryson GL, Yang H, Kalkman CJ, Wells GA, Beattie WS. The value of routine preoperative electrocardiography in predicting myocardial infarction after noncardiac surgery. Annals of Surgery. 2007;246(2):165-70. 
14. Sheth T, Chan M, Butler C, Chow B, Tandon V, Nagele P, et al. Prognostic capabilities of coronary computed tomographic angiography before non-cardiac surgery: prospective cohort study. BMJ-British Medical Journal. 2015;350.

15. Katsanos S, Babalis D, Kafkas N, Mavrogenis A, Leong D, Parissis J, et al. B-type natriuretic peptide vs. cardiac risk scores for prediction of outcome following major orthopedic surgery. Journal of Cardiovascular Medicine. 2015;16(6):465-71.

16. Mercantini P, Di Somma S, Magrini L, Nava AK, Scarinci A, La Torre M, et al. Preoperative Brain Natriuretic Peptide (BNP) Is a Better Predictor of Adverse Cardiac Events Compared to Preoperative Scoring System in Patients Who Underwent Abdominal Surgery. World Journal of Surgery. 2012;36(1):24-30.

17. Weber M, Luchner A, Manfred S, Mueller C, Liebetrau C, Schlitt A, et al. Incremental value of high-sensitive troponin $\mathrm{T}$ in addition to the revised cardiac index for peri-operative risk stratification in non-cardiac surgery. European Heart Journal. 2013;34(11):853-62.

18. Park K, Kim H-K, Kim Y-J, Cho G-Y, Kim K-H, Kim K-B, et al. Incremental prognostic value of early postoperative right ventricular systolic function in patients undergoing surgery for isolated severe tricuspid regurgitation. Heart. 2011;97(16):1319-25.

19. Cohn SL, Fernandez Ros N. Comparison of 4 Cardiac Risk Calculators in Predicting Postoperative Cardiac Complications After Noncardiac Operations. American Journal of Cardiology. 2018;121(1):125-30.

20. Markovic DZ, Jevtovic-Stoimenov T, Cosic V, Stosic B, Zivkovic BM, Jankovic RJ. Addition of biomarker panel improves prediction performance of American College of Surgeons National Surgical Quality Improvement Program (ACS NSQIP) calculator for cardiac risk assessment of elderly patients preparing for major non-cardiac surgery: a pilot study. Aging Clinical and Experimental Research. 2018;30(5):419-31.

21. Gupta PK, Gupta H, Sundaram A, Kaushik M, Fang X, Miller WJ, et al. Development and Validation of a Risk Calculator for Prediction of Cardiac Risk After Surgery. Circulation. 2011;124(4):381-U146.

22. Asuzu DT, Chao GF, Pei KY. Revised cardiac risk index poorly predicts cardiovascular complications after adhesiolysis for small bowel obstruction. Surgery. 2018;23:23.

23. van Waes JAR, Grobben RB, Nathoe HM, Kemperman H, de Borst GJ, Peelen LM, et al. OneYear Mortality, Causes of Death, and Cardiac Interventions in Patients with Postoperative Myocardial Injury. Anesthesia and Analgesia. 2016;123(1):29-37.

24. Devereaux PJ, Biccard BM, Chan MTV. High-Sensitivity Troponin Levels, Ischemia, and Mortality-Reply. Jama. 2017;318(9):865.

25. Devereaux PJ, Biccard BM, Sigamani A, Xavier D, Chan MTV, Srinathan SK, et al. Association of Postoperative High-Sensitivity Troponin Levels With Myocardial Injury and 30-Day Mortality Among Patients Undergoing Noncardiac Surgery. Jama. 2017;317(16):1642-51.

26. Ekeloef S, Alamili M, Devereaux PJ, Gogenur I. Troponin elevations after non-cardiac, nonvascular surgery are predictive of major adverse cardiac events and mortality: a systematic review and meta-analysis. British Journal of Anaesthesia. 2016;117(5):559-68.

27. Devereaux PJ, Chan MTV, Alonso-Coello P, Walsh M, Berwanger O, Villar JC, et al. Association between postoperative troponin levels and 30-day mortality among patients undergoing noncardiac surgery. JAMA - Journal of the American Medical Association. 2012;307(21):2295304. 
28. Mackey WC, Fleisher LA, Haider S, Sheikh S, Cappelleri JC, Lee WC, et al. Perioperative myocardial ischemic injury in high-risk vascular surgery patients: incidence and clinical significance in a prospective clinical trial. J Vasc Surg. 2006;43(3):533-8.

29. Kip KE, Hollabaugh K, Marroquin OC, Williams DO. The problem with composite end points in cardiovascular studies: the story of major adverse cardiac events and percutaneous coronary intervention. J Am Coll Cardiol. 2008;51(7):701-7.

30. Bronheim RS, Oermann EK, Bronheim DS, Caridi JM. Revised Cardiac Risk Index vs. ASA Status as a Predictor for Non-Cardiac Events Following Posterior Lumbar Decompression. World Neurosurgery. 2018;12:12.

31. Press MJ, Chassin MR, Wang J, Tuhrim S, Halm EA. Predicting medical and surgical complications of carotid endarterectomy - Comparing the risk indexes. Archives of Internal Medicine. 2006;166(8):914-20.

32. Ehlert BA, Najafian A, Orion KC, Malas MB, Black JH, III, Abularrage CJ. Validation of a modified Frailty Index to predict mortality in vascular surgery patients. Journal of Vascular Surgery. 2016;63(6):1595-+.

33. Makary MA, Segev DL, Pronovost PJ, Syin D, Bandeen-Roche K, Patel P, et al. Frailty as a Predictor of Surgical Outcomes in Older Patients. Journal of the American College of Surgeons. 2010;210(6):901-8.

34. Palladino R, Pennino F, Finbarr M, Millett C, Triassi M. Multimorbidity And Health Outcomes In Older Adults In Ten European Health Systems, 2006-15. Health Aff (Millwood). 2019;38(4):61323.

35. Wolff JL, Starfield B, Anderson G. Prevalence, expenditures, and complications of multiple chronic conditions in the elderly. Arch Intern Med. 2002;162(20):2269-76.

36. Mayhew D, Mendonca V, Murthy BVS. A review of ASA physical status - historical perspectives and modern developments. Anaesthesia. 2019;74(3):373-9.

37. Moons KG, de Groot JA, Bouwmeester W, Vergouwe Y, Mallett S, Altman DG, et al. Critical appraisal and data extraction for systematic reviews of prediction modelling studies: the CHARMS checklist. PLoS Med. 2014;11(10):e1001744.

38. Debray TP, Damen JA, Snell KI, Ensor J, Hooft L, Reitsma JB, et al. A guide to systematic review and meta-analysis of prediction model performance. Bmj. 2017;356:i6460.

39. Riley RD, Van der Windt DA, Croft P, Moons KG. Prognosis Research in Healthcare: Concepts, Methods, and Impact. Oxford: Oxford University Press 2019.

40. Vetrugno L, Langiano N, Gisonni R, Rizzardo A, Venchiarutti PE, Divella M, et al. Prediction of early postoperative major cardiac events after elective orthopedic surgery: the role of B-type natriuretic peptide, the revised cardiac risk index, and ASA class. Bmc Anesthesiology. 2014;14.

41. Gualandro DM, Puelacher C, LuratiBuse G, Lampart A, Strunz C, Cardozo FA, et al. Comparison of high-sensitivity cardiac troponin I and T for the prediction of cardiac complications after non-cardiac surgery. American Heart Journal. 2018;203:67-73.

42. Feringa HHH, Schouten O, Dunkelgrun M, Bax JJ, Boersma E, Elhendy A, et al. Plasma $\mathrm{N}$-terminal pro-B-type natriuretic peptide as long-term prognostic marker after major vascular surgery. Heart. 2007;93(2):226-31.

43. Biomarkers and surrogate endpoints: preferred definitions and conceptual framework. Clin Pharmacol Ther. 2001;69(3):89-95. 
44. Rohrig R, Junger A, Hartmann B, Klasen J, Quinzio L, Jost A, et al. The incidence and prediction of automatically detected intraoperative cardiovascular events in noncardiac surgery. Anesthesia and Analgesia. 2004;98(3):569-77.

45. Subramaniam B, Meroz Y, Talmor D, Pomposelli FB, Berlatzky Y, Landesberg G. A LongTerm Survival Score Improves Preoperative Prediction of Survival Following Major Vascular Surgery. Annals of Vascular Surgery. 2011;25(2):197-203.

46. Geersing GJ, Bouwmeester W, Zuithoff P, Spijker R, Leeflang M, Moons KG. Search filters for finding prognostic and diagnostic prediction studies in Medline to enhance systematic reviews. PLoS One. 2012;7(2):e32844.

47. Goldman L, Caldera DL, Nussbaum SR, Southwick FS, Krogstad D, Murray B, et al. Multifactorial index of cardiac risk in noncardiac surgical procedures. N Engl J Med. 1977;297(16):845-50.

48. Detsky AS, Abrams HB, Forbath N, Scott JG, Hilliard JR. Cardiac assessment for patients undergoing noncardiac surgery. A multifactorial clinical risk index. Arch Intern Med. 1986;146(11):2131-4.

49. Vernooij LMDJVKWAMK, Peelen LM. The added value of different biomarkers to the Revised Cardiac Risk Index to predict major adverse cardiac events and all-cause mortality after noncardiac surgery. Cochrane Database of Systematic Reviews. 2018(10).

50. Moons KGM, Wolff RF, Riley RD, Whiting PF, Westwood M, Collins GS, et al. PROBAST: A Tool to Assess Risk of Bias and Applicability of Prediction Model Studies: Explanation and Elaboration. Ann Intern Med. 2019;170(1):W1-w33.

51. Wolff RF, Moons KGM, Riley RD, Whiting PF, Westwood M, Collins GS, et al. PROBAST: A Tool to Assess the Risk of Bias and Applicability of Prediction Model Studies. Ann Intern Med. 2019;170(1):51-8.

52. Steyerberg EW. Clinical Prediction Models: A Practical Approach to Development, Validation, and Updating: Springer New York; 2008.

53. Harrell FE. Regression Modeling Strategies: With Applications to Linear Models, Logistic and Ordinal Regression, and Survival Analysis: Springer International Publishing; 2015.

54. Collins GS, Reitsma JB, Altman DG, Moons KG. Transparent Reporting of a multivariable prediction model for Individual Prognosis Or Diagnosis (TRIPOD). Ann Intern Med. 2015;162(10):735-6.

55. Moons KG, Altman DG, Reitsma JB, loannidis JP, Macaskill P, Steyerberg EW, et al. Transparent Reporting of a multivariable prediction model for Individual Prognosis or Diagnosis (TRIPOD): explanation and elaboration. Ann Intern Med. 2015;162(1):W1-73.

56. Heus P, Damen J, Pajouheshnia R, Scholten R, Reitsma JB, Collins GS, et al. Poor reporting of multivariable prediction model studies: towards a targeted implementation strategy of the TRIPOD statement. BMC Med. 2018;16(1):120.

57. Bouwmeester W, Zuithoff NP, Mallett S, Geerlings MI, Vergouwe Y, Steyerberg EW, et al. Reporting and methods in clinical prediction research: a systematic review. PLoS Med. 2012;9(5):1-12.

58. Collins GS, de Groot JA, Dutton S, Omar O, Shanyinde M, Tajar A, et al. External validation of multivariable prediction models: a systematic review of methodological conduct and reporting. BMC Med Res Methodol. 2014;14:40. 
59. Collins GS, Omar O, Shanyinde M, Yu LM. A systematic review finds prediction models for chronic kidney disease were poorly reported and often developed using inappropriate methods. J Clin Epidemiol. 2013;66(3):268-77.

60. Mallett S, Royston P, Dutton S, Waters R, Altman DG. Reporting methods in studies developing prognostic models in cancer: a review. BMC Med. 2010;8:20.

61. Laupacis A, Sekar N, Stiell IG. Clinical prediction rules. A review and suggested modifications of methodological standards. Jama. 1997;277(6):488-94.

62. Viechtbauer W. Conducting Meta-Analyses in R with the metafor Package. 2010. 2010;36(3):48.

63. Debray TP, de Jong VMT. metamisc: Diagnostic and Prognostic Meta-Analysis. 2019.

64. Riley RD, Higgins JP, Deeks JJ. Interpretation of random effects meta-analyses. Bmj. 2011;342:d549.

65. Iorio A, Spencer FA, Falavigna M, Alba C, Lang E, Burnand B, et al. Use of GRADE for assessment of evidence about prognosis: rating confidence in estimates of event rates in broad categories of patients. Bmj. 2015;350:h870.

66. Foroutan F, Guyatt G, Zuk V, Vandvik PO, Alba AC, Mustafa R, et al. Use of GRADE for assessment of evidence about prognostic factors: rating certainty in identification of groups of patients with different absolute risks. J Clin Epidemiol. 2020.

67. Yancy CW, Jessup M, Bozkurt B, Butler J, Casey DE, Jr., Drazner MH, et al. 2013 ACCF/AHA guideline for the management of heart failure: a report of the American College of Cardiology Foundation/American Heart Association Task Force on practice guidelines. Circulation. 2013;128(16):e240-327.

68. Wijeysundera DN, Pearse RM, Shulman MA, Abbott TEF, Torres E, Ambosta A, et al. Assessment of functional capacity before major non-cardiac surgery: an international, prospective cohort study. Lancet. 2018;391(10140):2631-40.

69. Mair J, Lindahl B, Hammarsten O, Muller C, Giannitsis E, Huber K, et al. How is cardiac troponin released from injured myocardium? Eur Heart J Acute Cardiovasc Care. 2018;7(6):553-60.

70. Thygesen K, Alpert JS, Jaffe AS, Chaitman BR, Bax JJ, Morrow DA, et al. Fourth Universal Definition of Myocardial Infarction (2018). Circulation. 2018;138(20):e618-e51.

71. Mauermann E, Bolliger D, Seeberger E, Puelacher C, Corbiere S, Filipovic M, et al. Incremental Value of Preoperative Copeptin for Predicting Myocardial Injury. Anesthesia and Analgesia. 2016;123(6):1363-71.

72. Schrimpf C, Gillmann H-J, Sahlmann B, Meinders A, Larmann J, Wilhelmi M, et al. Renal function interferes with copeptin in prediction of major adverse cardiac events in patients undergoing vascular surgery. PLoS ONE [Electronic Resource]. 2015;10(4):e0123093.

73. Jarai R, Mahla E, Perkmann T, Jarai R, Archan S, Tentzeris I, et al. Usefulness of Pre-Operative Copeptin Concentrations to Predict Post-Operative Outcome After Major Vascular Surgery. American Journal of Cardiology. 2011;108(8):1188-95.

74. Rodseth RN, Buse GAL, Bolliger D, Burkhart CS, Cuthbertson BH, Gibson SC, et al. The Predictive Ability of Pre-Operative B-Type Natriuretic Peptide in Vascular Patients for Major Adverse Cardiac Events An Individual Patient Data Meta-Analysis. Journal of the American College of Cardiology. 2011;58(5):522-9. 
75. Biccard BM, Naidoo P. The role of brain natriuretic peptide in prognostication and reclassification of risk in patients undergoing vascular surgery. Anaesthesia. 2011;66(5):37985.

76. Pepys MB, Hirschfield GM. C-reactive protein: a critical update. J Clin Invest. 2003;111(12):180512.

77. James S, Jhanji S, Smith A, O'Brien G, Fitzgibbon M, Pearse RM. Comparison of the prognostic accuracy of scoring systems, cardiopulmonary exercise testing, and plasma biomarkers: a single-centre observational pilot study. British Journal of Anaesthesia. 2014;112(3):491-7.

78. Ray MJ, Calabro LJ, Sirisena T, Crawford SA, Crawford RW, Walters DL. Pre-operative plateletbound CD40 ligand is probably associated with peri-operative cardiac events in hip and knee arthroplasty. European Journal of Clinical Investigation. 2010;40(6):497-503.

79. Bilimoria KY, Liu Y, Paruch JL, Zhou L, Kmiecik TE, Ko CY, et al. Development and evaluation of the universal ACS NSQIP surgical risk calculator: a decision aid and informed consent tool for patients and surgeons. J Am Coll Surg. 2013;217(5):833-42.e1-3.

80. Copeland CC, Young A, Grogan T, Gabel E, Dhillon A, Gudzenko V. Preoperative risk stratification of critically ill patients. Journal of Clinical Anesthesia. 2017;39:122-7.

81. Alrezk R, Jackson N, Al Rezk M, Elashoff R, Weintraub N, Elashoff D, et al. Derivation and Validation of a Geriatric-Sensitive Perioperative Cardiac Risk Index. Journal of the American Heart Association. 2017;6(11).

82. Pantoja Muñoz HJ, Fernández Ramos H, Guevara Tovar WL. Sensitivity, specificity and predictive values of the Goldman, Detsky and Lee cardiac indices. Revista Colombiana de Anestesiologia. 2014;42(3):184-91.

83. Jammer I, Wickboldt N, Sander M, Smith A, Schultz MJ, Pelosi P, et al. Standards for definitions and use of outcome measures for clinical effectiveness research in perioperative medicine: European Perioperative Clinical Outcome (EPCO) definitions: a statement from the ESAESICM joint taskforce on perioperative outcome measures. Eur J Anaesthesiol. 2015;32(2):88105.

84. Sabate S, Mases A, Guilera N, Canet J, Castillo J, Orrego C, et al. Incidence and predictors of major perioperative adverse cardiac and cerebrovascular events in non-cardiac surgery. British Journal of Anaesthesia. 2011;107(6):879-90.

85. Myles PS, Grocott MP, Boney O, Moonesinghe SR. Standardizing end points in perioperative trials: towards a core and extended outcome set. Br J Anaesth. 2016;116(5):586-9.

86. Barnes J, Hunter J, Harris S, Shankar-Hari M, Diouf E, Jammer I, et al. Systematic review and consensus definitions for the Standardised Endpoints in Perioperative Medicine (StEP) initiative: infection and sepsis. Br J Anaesth. 2019;122(4):500-8.

87. Buggy DJ, Freeman J, Johnson MZ, Leslie K, Riedel B, Sessler DI, et al. Systematic review and consensus definitions for standardised endpoints in perioperative medicine: postoperative cancer outcomes. Br J Anaesth. 2018;121(1):38-44.

88. Haller G, Bampoe S, Cook T, Fleisher LA, Grocott MPW, Neuman M, et al. Systematic review and consensus definitions for the Standardised Endpoints in Perioperative Medicine initiative: clinical indicators. Br J Anaesth. 2019;123(2):228-37.

89. Moonesinghe SR, Jackson AIR, Boney O, Stevenson N, Chan MTV, Cook TM, et al. Systematic review and consensus definitions for the Standardised Endpoints in Perioperative Medicine initiative: patient-centred outcomes. Br J Anaesth. 2019;123(5):664-70. 
90. Myles PS, Boney O, Botti M, Cyna AM, Gan TJ, Jensen MP, et al. Systematic review and consensus definitions for the Standardised Endpoints in Perioperative Medicine (StEP) initiative: patient comfort. Br J Anaesth. 2018;120(4):705-11.

91. Moons KG, van Es GA, Michel BC, Buller HR, Habbema JD, Grobbee DE. Redundancy of single diagnostic test evaluation. Epidemiology. 1999;10(3):276-81. 


\section{SUPPLEMENTAL MATERIAL}

\section{Supplemental section 1. MEDLINE Ovid search strategy}

1. ("Revised Cardiac risk index" or RCRI or "Lee index" or "Lee-index" or "Lee's index" or "revised goldman index" or goldman or detsky or LCRI or RCl or "revised cardiac index" or "pre-operative variable*" or "preoperative variable*" or "revised cardiac risk" or "cardiac risk factor"). ti,ab,kf.

2. Reproducibility of Results/or calibration/ or Area Under Curve/ orValidation Studies.pt. or (validat* or stratification or overfit* or overpredict* or underfit* or underpredict* or overestimation or underestimation or pooled or recalibration or re-calibration or calibration or discrimination or cohort or discriminate or c-statistic* or "c statistic" or "Area under the curve*" or AUC or Indices or Algorithm or Multivariable or "added value" or incremental or "receiver operating curve" or roc or "receiver operating characteristic" or "c index" or "c-index" or "predictive accuracy" or "prognostic accuracy" or "reclassifi" or "prognostic value" or "predictive value" or MACE).ti,ab,kf.

3. 1 and 2

4. (exp animals/ not humans/) or (equine or cattle or bovine or canine or mice or mouse or rat or rats or guinea-pig* or dog).ti.

5. 3 not 4

\section{Supplemental section 2. Ovid Embase search strategy}

1. ("Revised Cardiac risk index" or RCRI or "Lee index" or "Lee-index" or "Lee's index" or "revised goldman index" or goldman or detsky or LCRI or RCl or "revised cardiac index" or "pre-operative variable*" or "preoperative variable" or "revised cardiac risk" or "cardiac risk factor").ti,ab,kw.

2. reproducibility/orvalidation study/orvalidation process/or calibration/or area under the curve/ or (validat ${ }^{\star}$ or stratification or overfit* or overpredict $^{\star}$ or underfit* or underpredict $^{\star}$ or overestimation or underestimation or pooled or recalibration or re-calibration or calibration or discrimination or cohort or discriminate or c-statistic* or "c statistic" or "Area under the curve " or AUC or Indices or Algorithm orMultivariable or "added value" or incremental or "receiver operating curve" or roc or "receiver operating characteristic" or "c index" or "c-index" or "predictive accuracy" or "prognostic accuracy" or "reclassifi" or "prognostic value" or "predictive value" or MACE).ti,ab,kw.

3. 1 and 2

4. ((exp experimental organism/ or animal tissue/ or animal cell/ or exp animal disease/ or exp carnivore disease/ or exp bird/ or exp experimental animal welfare/ or exp animal husbandry/ or animal behavior/ or exp animal cell culture/ or exp mammalian disease/ or exp mammal/ or exp marine species/ or nonhuman/or animal.hw.) not human/) or (equine or cattle or bovine or canine or mice or mouse or rat or rats or guinea-pig* or dog).ti.

5. 3 not 4

6. limit 5 to (conference abstract or conference paper or "conference review")

7. 5 not 6 


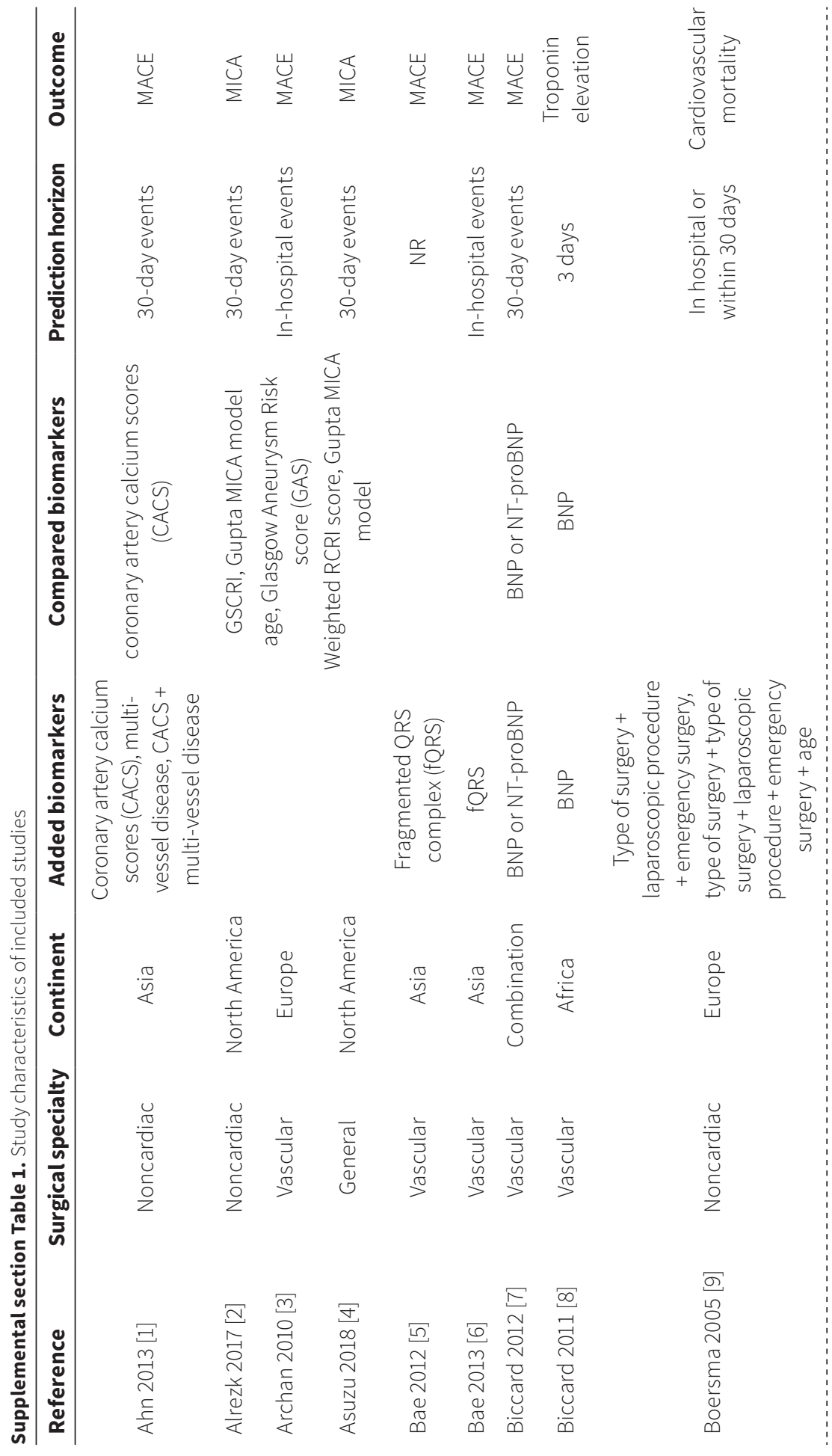


Chapter 5

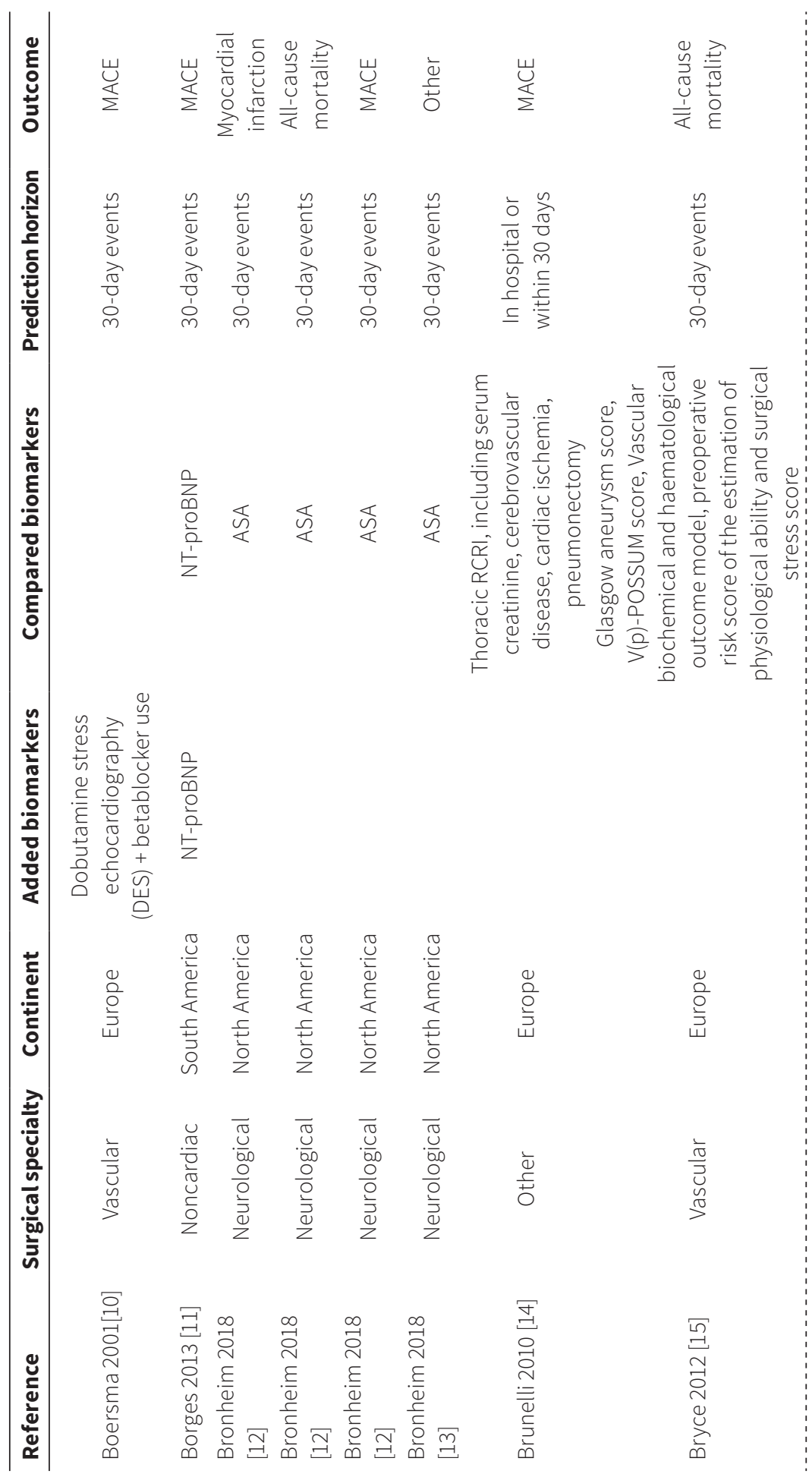




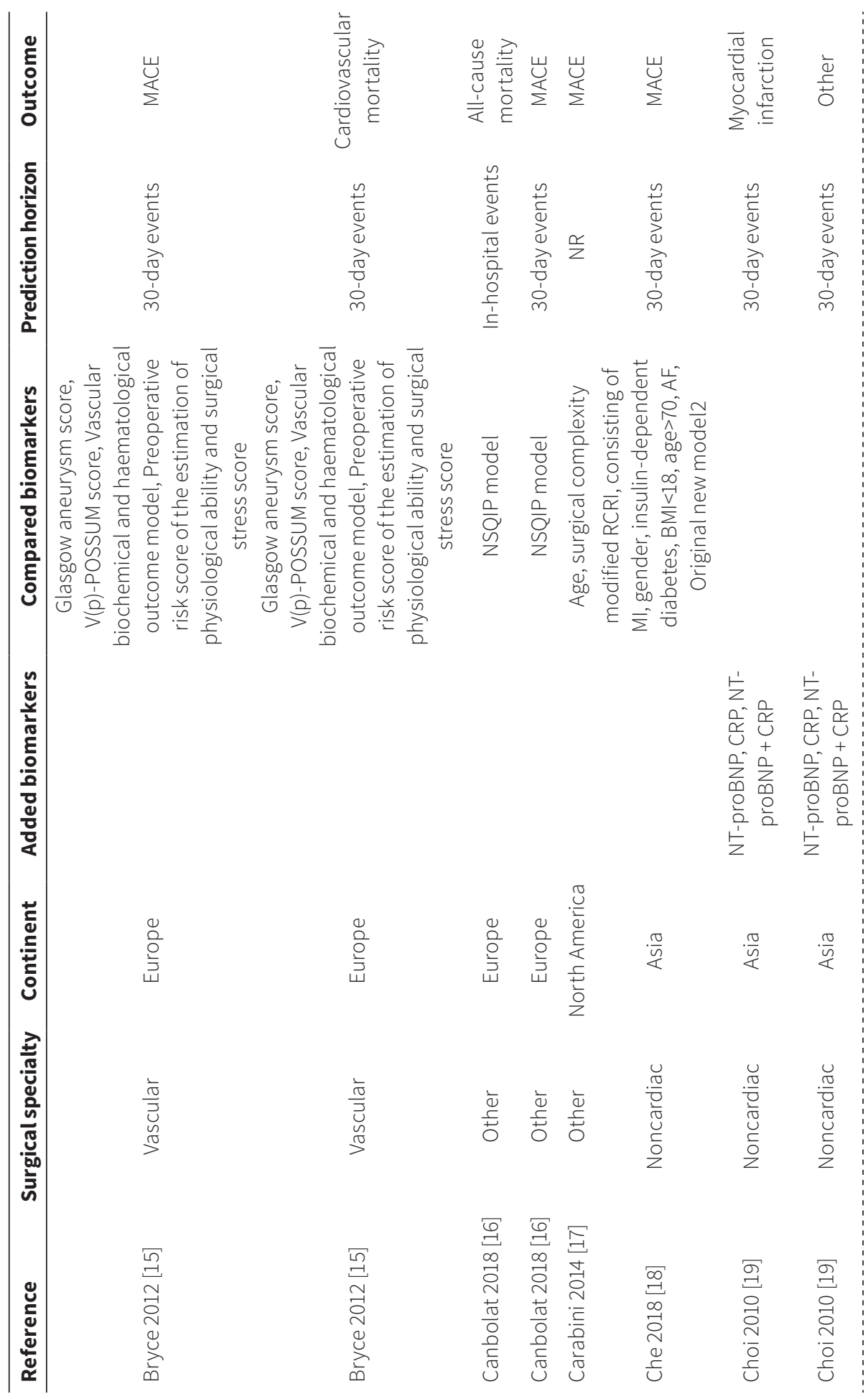




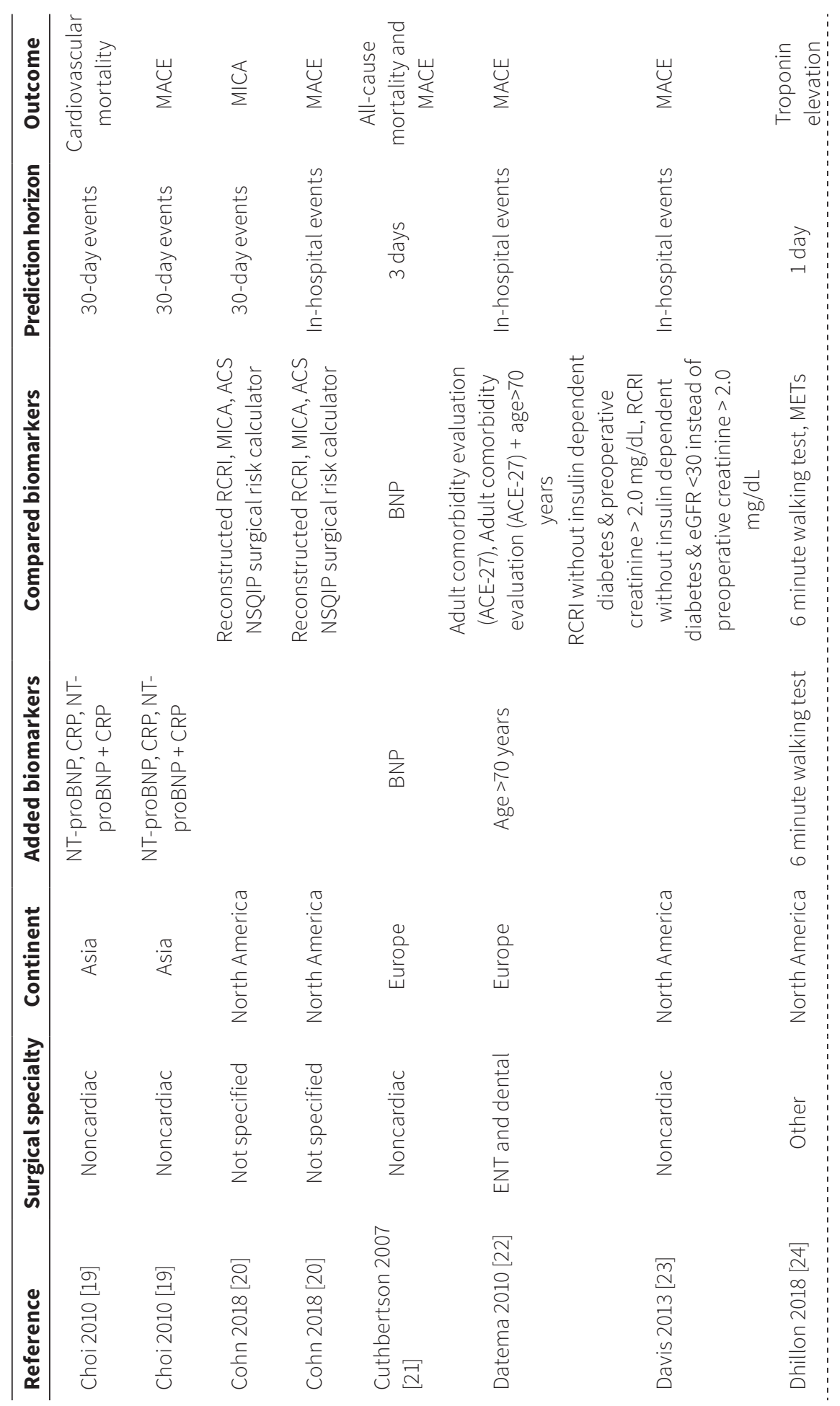




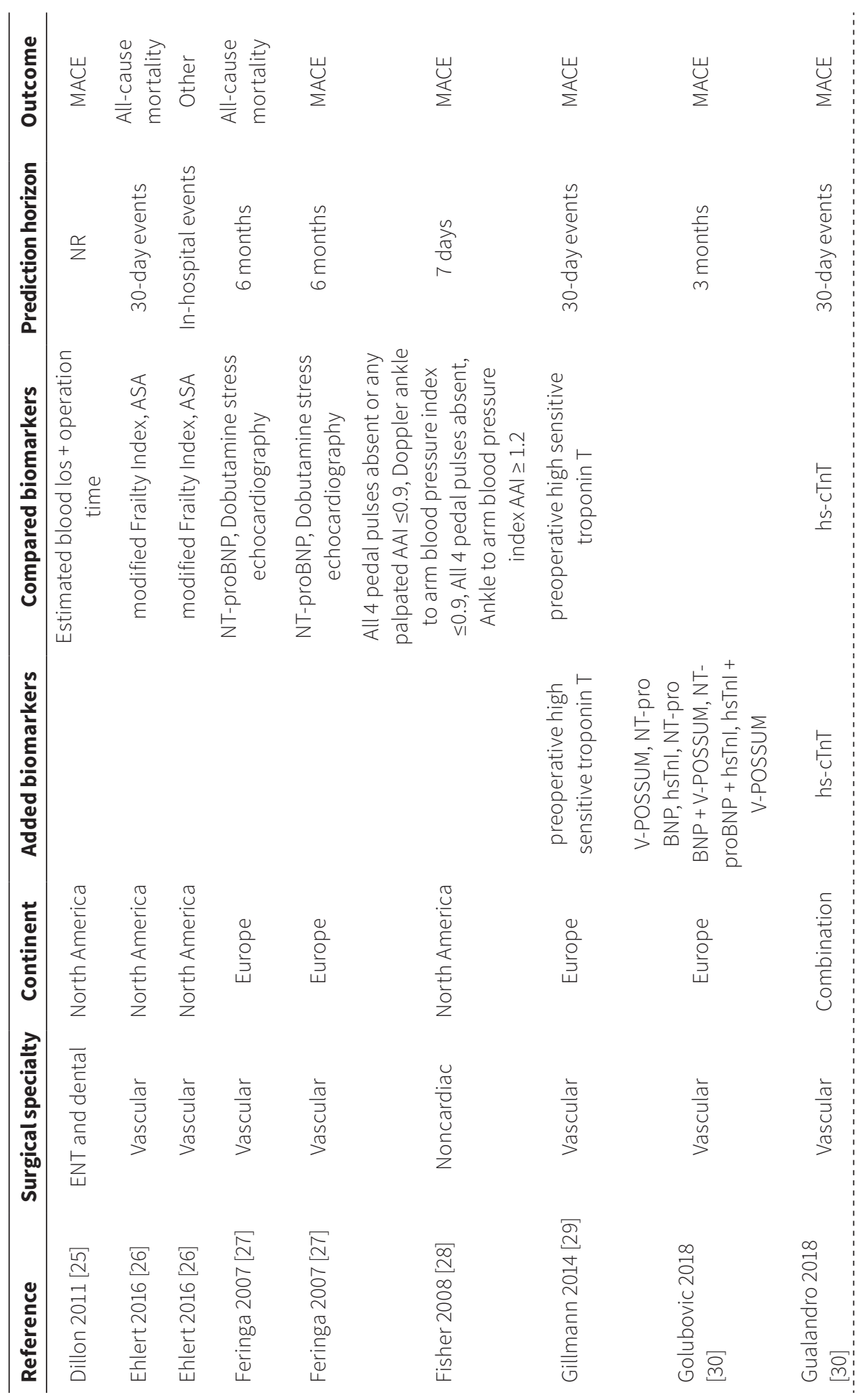




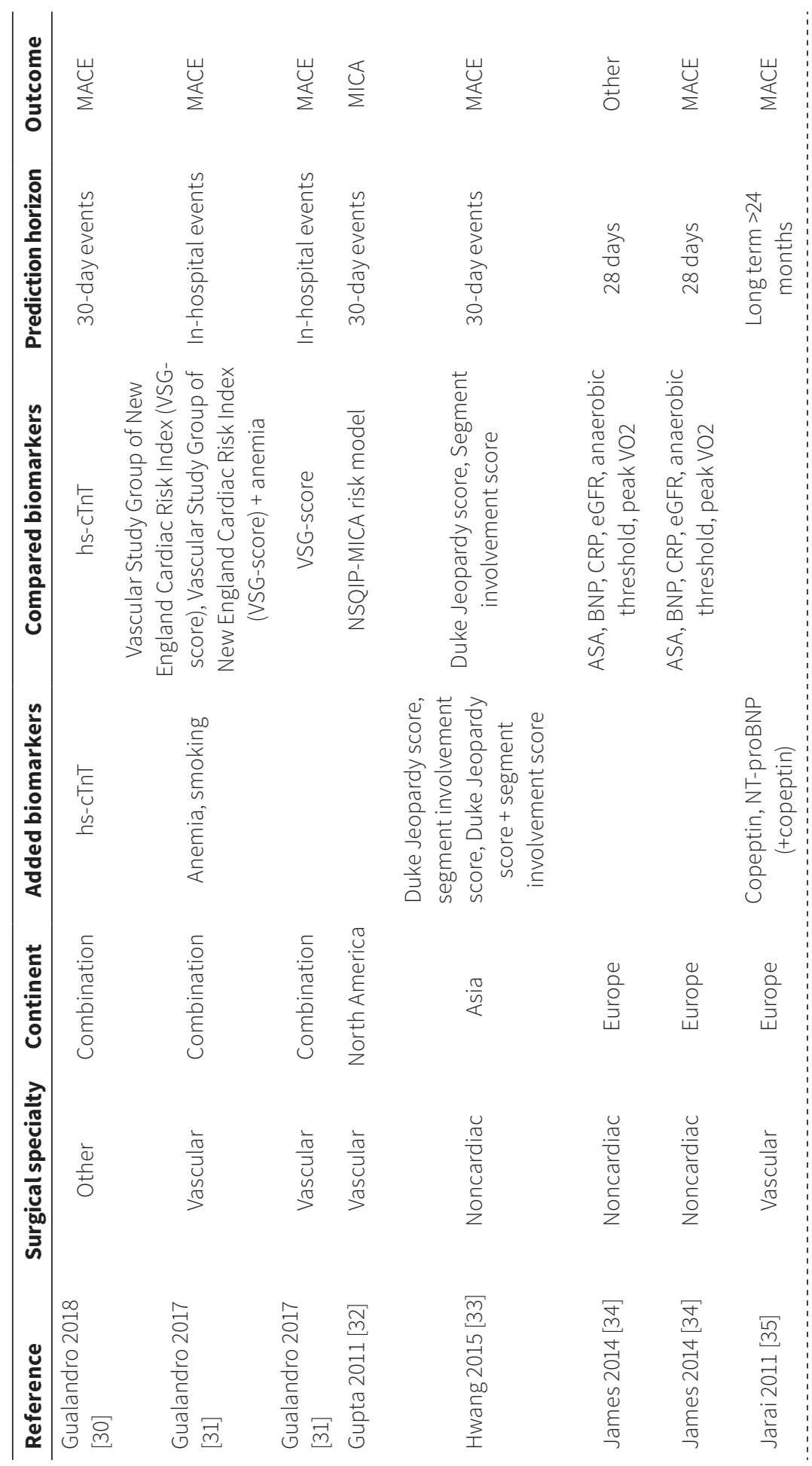




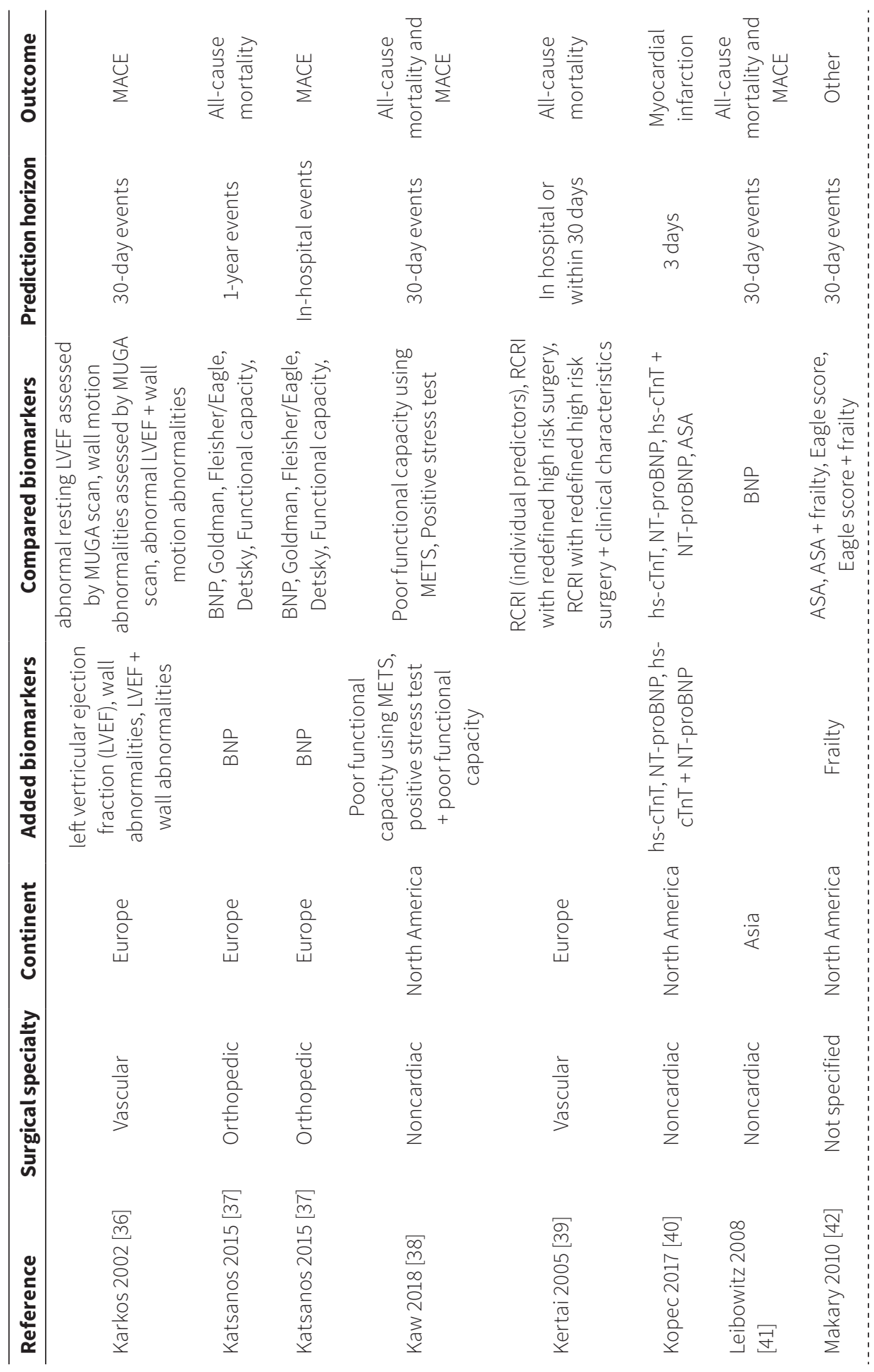




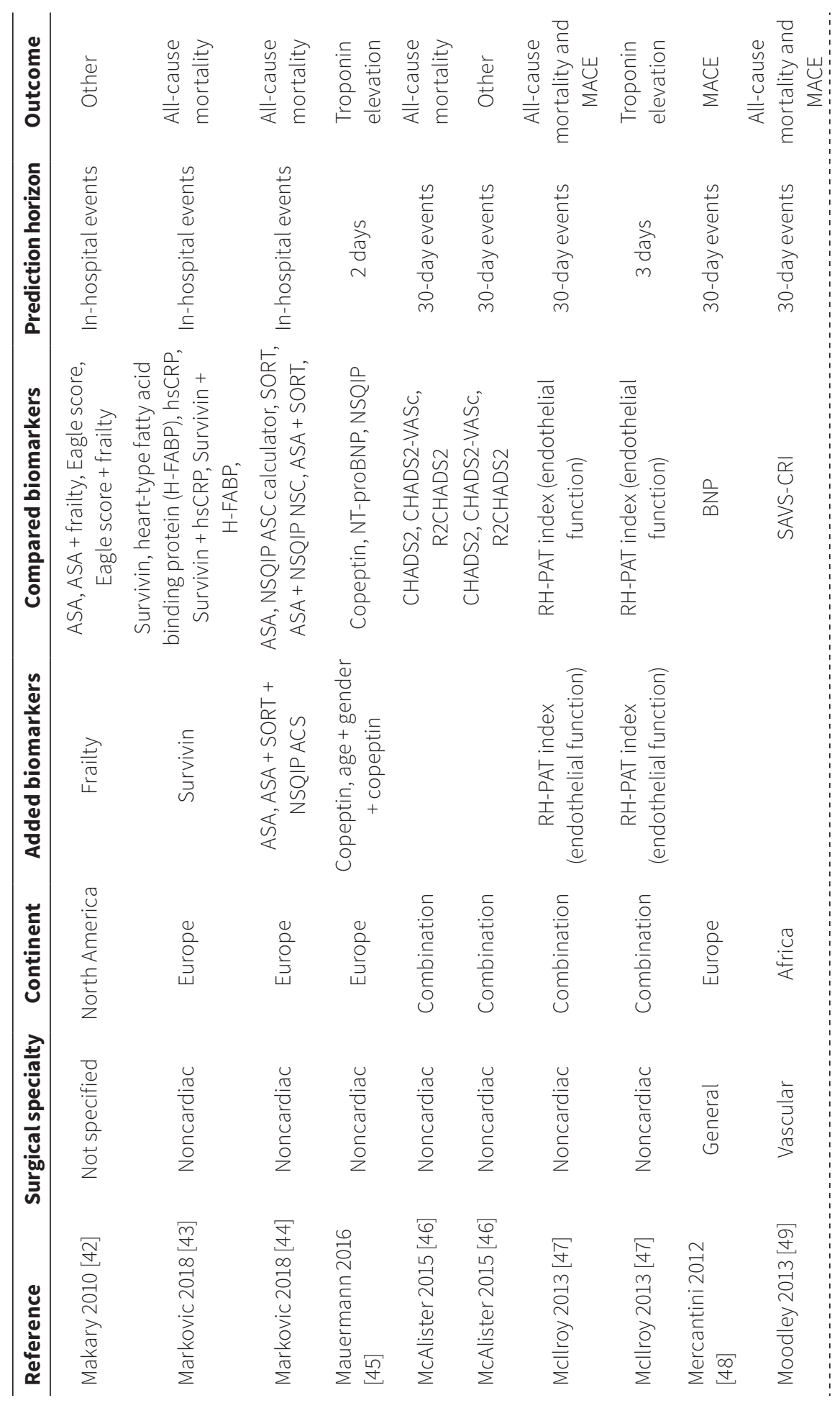




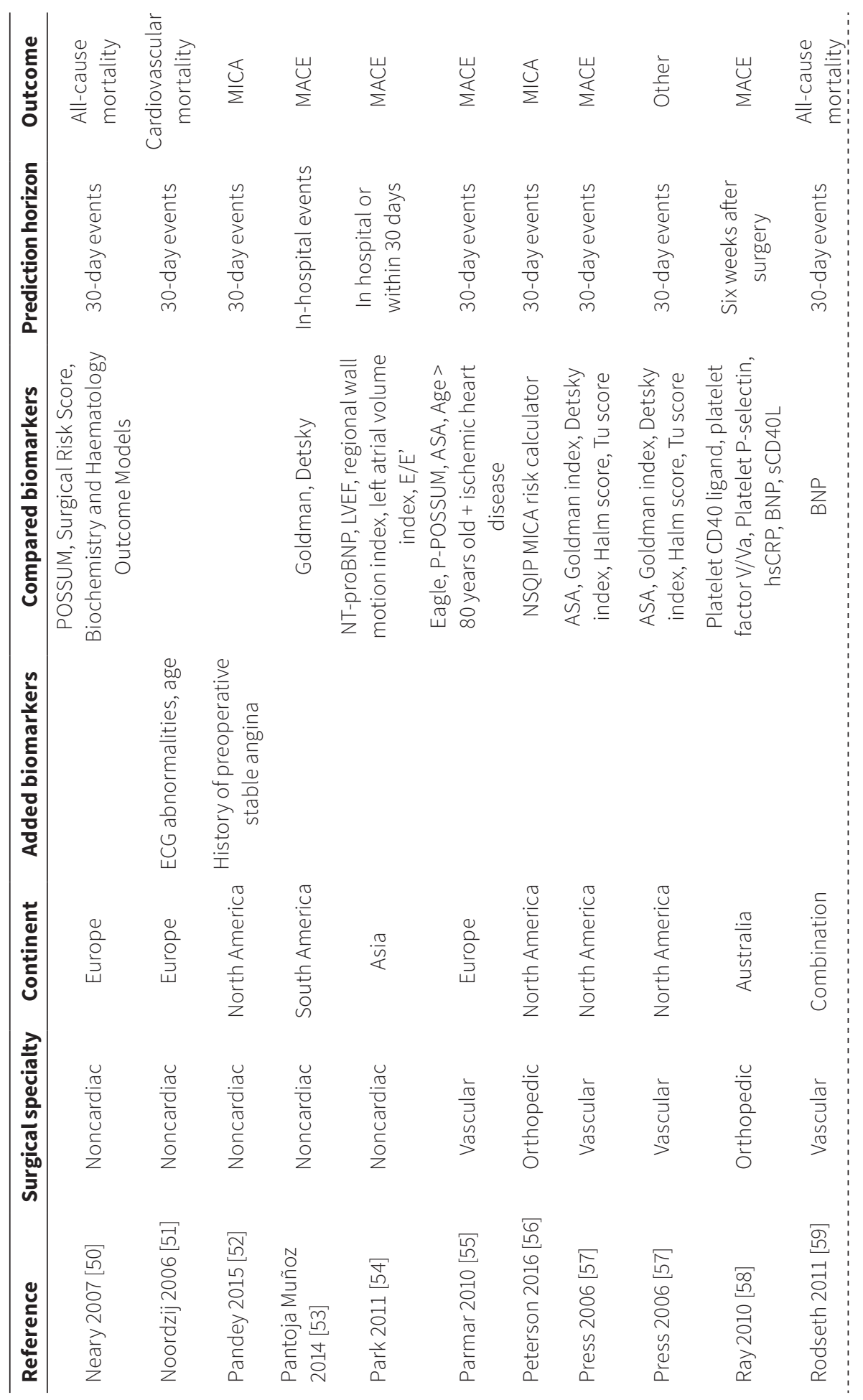




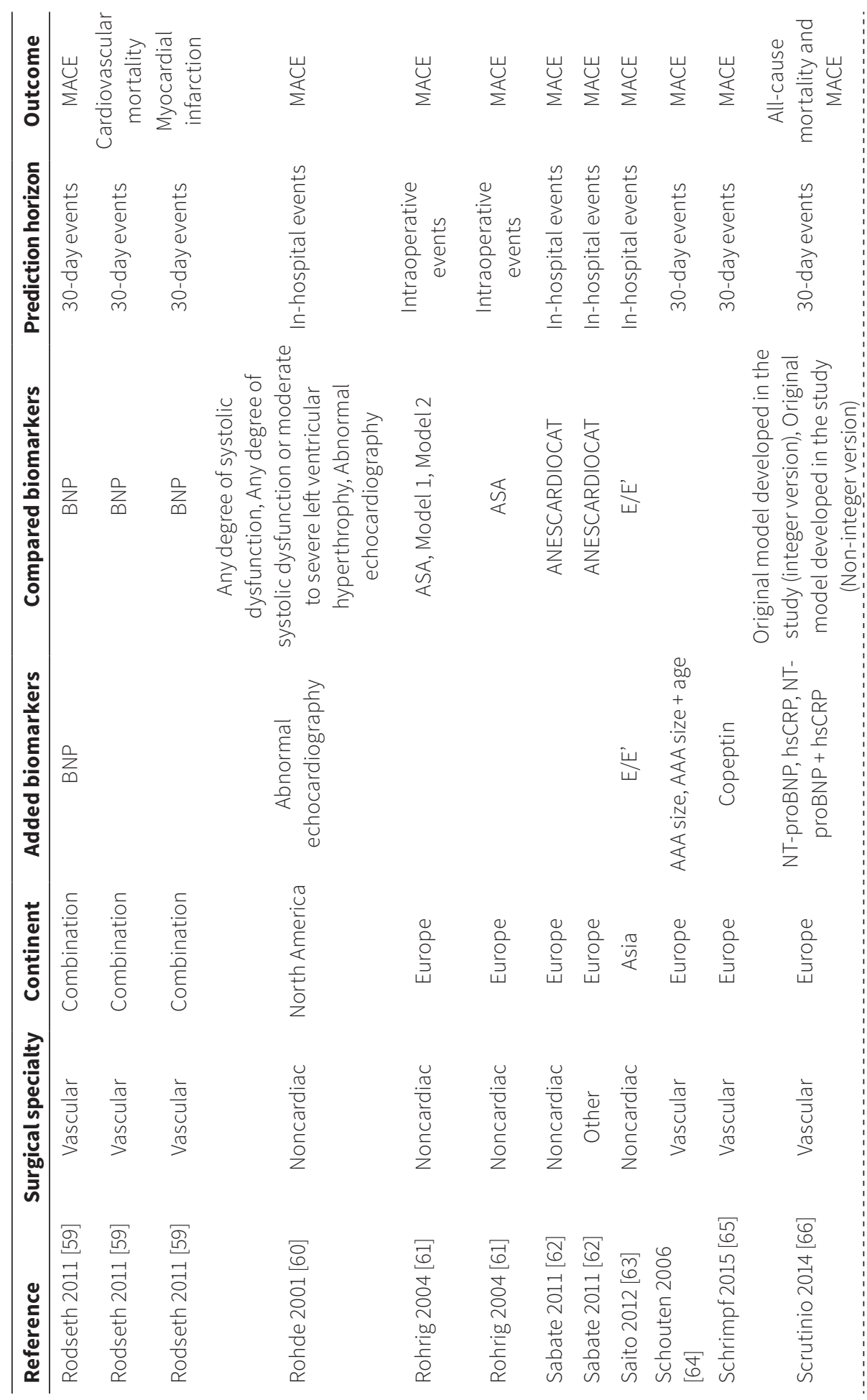




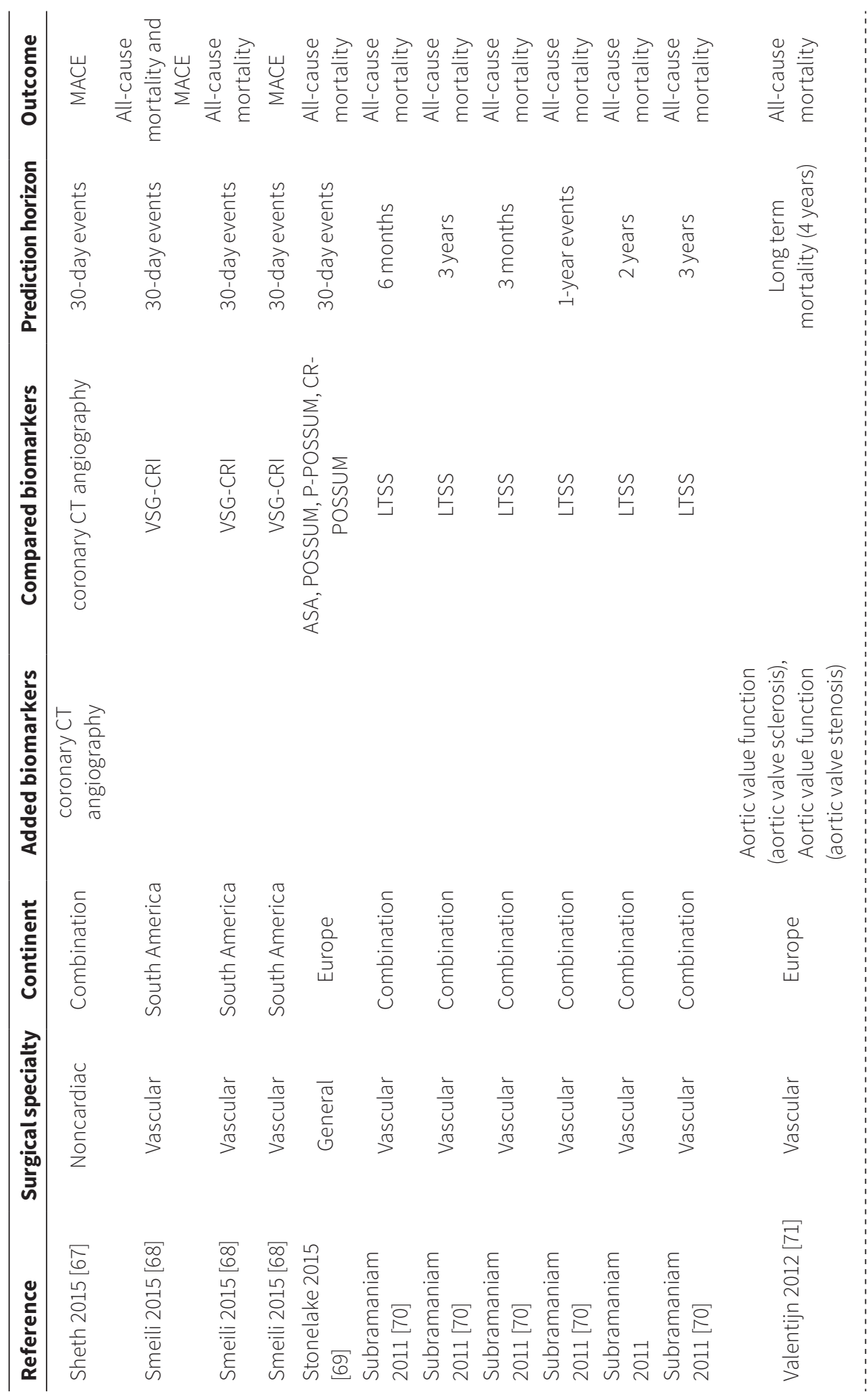




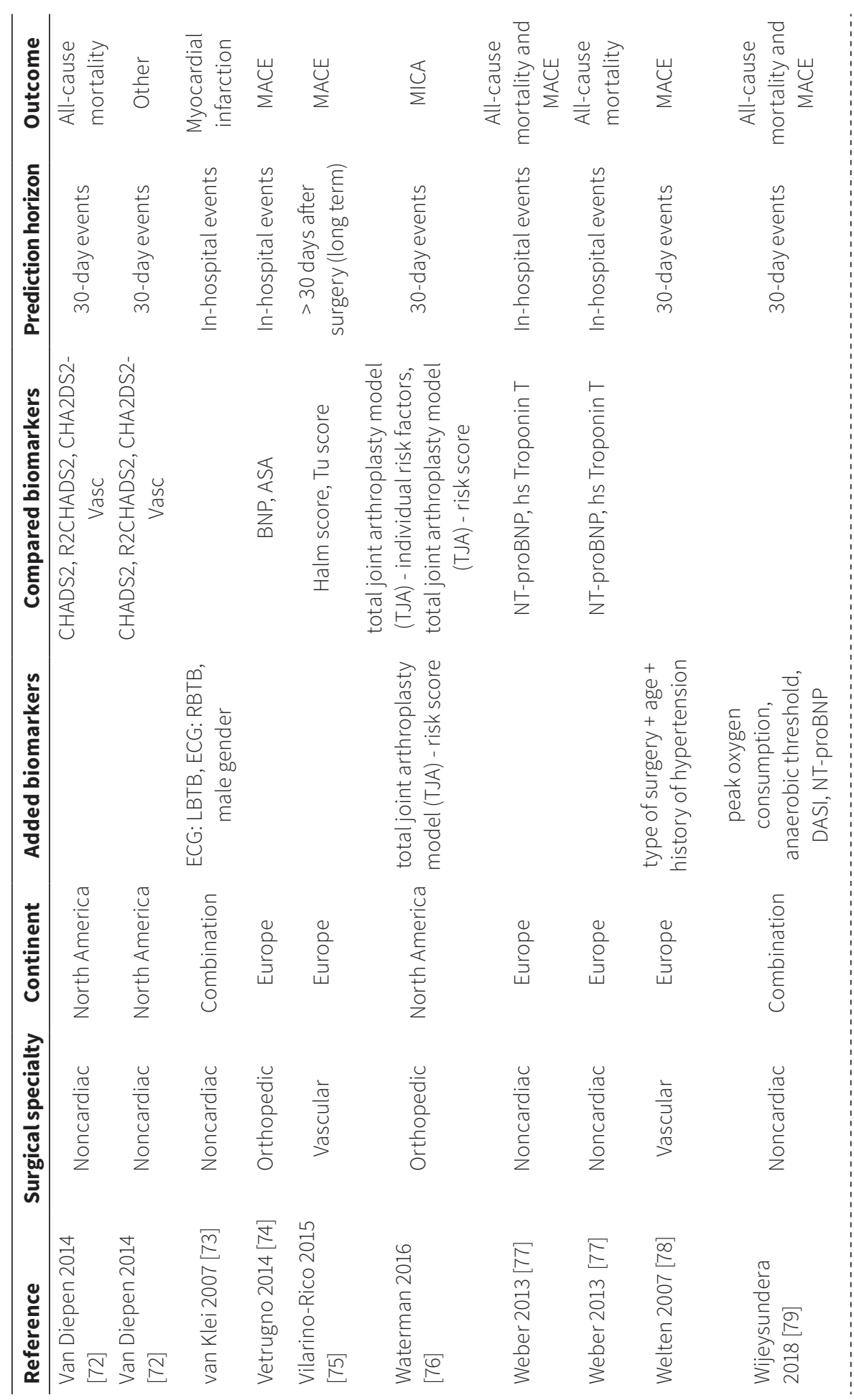




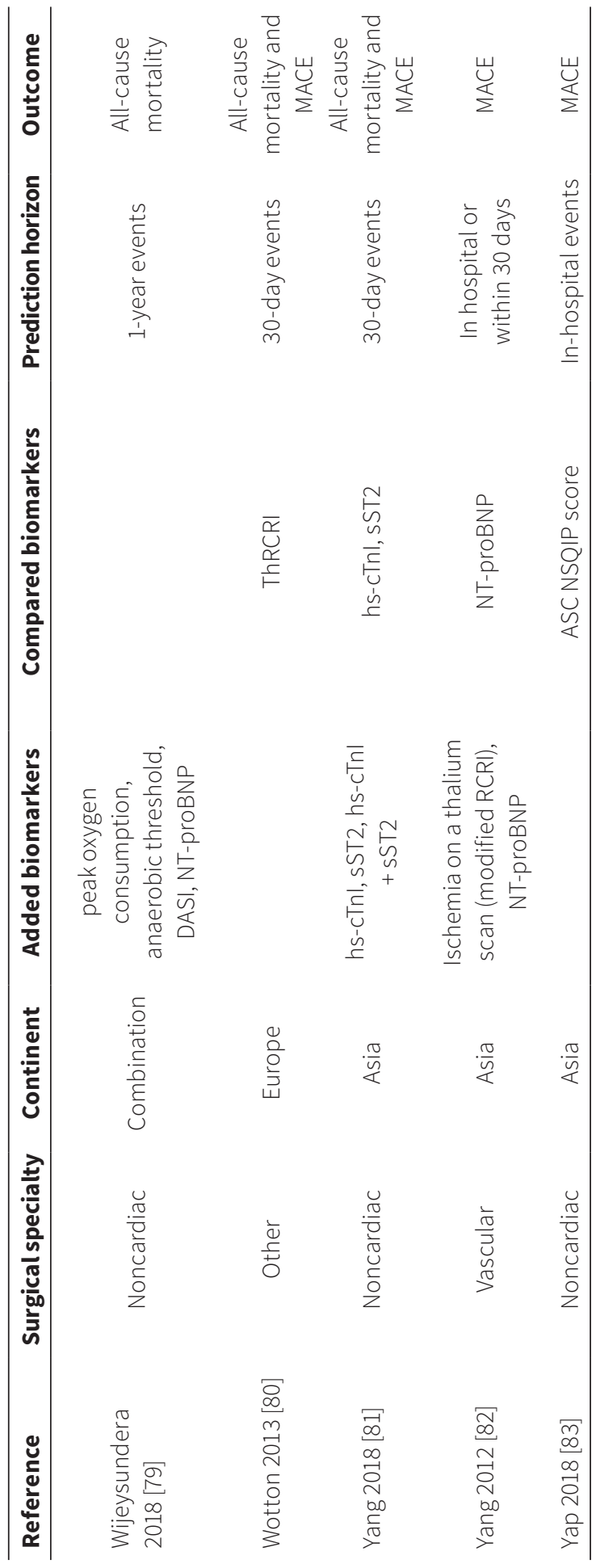


Supplemental section 4. List of included references

1. Ahn JH, Park JR, Min JH, Sohn JT, Hwang SJ, Park Y, Koh JS, Jeong YH, Kwak CH, Hwang JY: Risk stratification using computed tomography coronary angiography in patients undergoing intermediate-risk noncardiac surgery. J Am Coll Cardiol 2013, 61(6):661-668.

2. Alrezk R, Jackson N, Al Rezk M, Elashoff R, Weintraub N, Elashoff D, Fonarow GC: Derivation and Validation of a Geriatric-Sensitive Perioperative Cardiac Risk Index. Journal of the American Heart Association 2017, 6(11).

3. Archan S, Roscher CR, Fairman RM, Fleisher LA: Revised Cardiac Risk Index (Lee) and Perioperative Cardiac Events as Predictors of Long-term Mortality in Patients Undergoing Endovascular Abdominal Aortic Aneurysm Repair. Journal of Cardiothoracic and Vascular Anesthesia 2010, 24(1):84-90.

4. Asuzu DT, Chao GF, Pei KY: Revised cardiac risk index poorly predicts cardiovascular complications after adhesiolysis for small bowel obstruction. Surgery 2018, 23:23.

5. Bae MH, Choi WS, Kim KH, Park SH, Kim HW, Lee JH, Lee S-W, Yang DH, Park HS, Cho Y et al: The implications of a fragmented QRS complex and newly reclassified revised cardiac risk index including fragmented QRS in patients undergoing non-cardiac vascular surgery. International Journal of Cardiology 2012, 157(2):276-278.

6. Bae MH, Jang SY, Choi WS, Kim KH, Park SH, Lee JH, Kim HK, Yang DH, Huh S, Park HS et al: A New Revised Cardiac Risk Index Incorporating Fragmented QRS Complex as a Prognostic Marker in Patients Undergoing Noncardiac Vascular Surgery. American Journal of Cardiology 2013, 112(1):122-127.

7. Biccard BM, Buse GAL, Burkhart C, Cuthbertson BH, Filipovic M, Gibson SC, Mahla E, Leibowitz DW, Rodseth RN: The influence of clinical risk factors on pre-operative B-type natriuretic peptide risk stratification of vascular surgical patients. Anaesthesia 2012, 67(1):55-59.

8. Biccard BM, Naidoo P: The role of brain natriuretic peptide in prognostication and reclassification of risk in patients undergoing vascular surgery. Anaesthesia 2011, 66(5):379385.

9. Boersma E, Kertai MD, Schouten O, Bax JJ, Noordzij P, Steyerberg EW, Schinkel AFL, van Santen M, Simoons ML, Thomson IR et al: Perioperative cardiovascular mortality in noncardiac surgery: Validation of the Lee cardiac risk index. American Journal of Medicine 2005, 118(10):1134-1141.

10. Boersma E, Poldermans D, Bax JJ, Steyerberg EW, Thomson IR, Banga JD, van de Ven LLM, van Urk H, Roelandt J, Grp DS: Predictors of cardiac events after major vascular surgery Role of clinical characteristics, dobutamine echocardiography, and beta-blocker therapy. Jama-Journal of the American Medical Association 2001, 285(14):1865-1873.

11. Borges FK, Furtado MV, Webber Rossini AP, Bertoluci C, Gonzalez VL, Bertoldi EG, Grutcki DM, Rech LG, Magalhaes M, Polanczyk CA: Prognostic Value of Perioperative N-Terminal Pro-B-Type Natriuretic Peptide in Noncardiac Surgery. Arquivos Brasileiros De Cardiologia 2013, 100(6):561-570.

12. Bronheim RS, Oermann EK, Bronheim DS, Caridi JM: Revised Cardiac Risk Index as a Predictor for Myocardial Infarction and Cardiac Arrest Following Posterior Lumbar Decompression. Spine 2018, 12:12. 
13. Bronheim RS, Oermann EK, Bronheim DS, Caridi JM: Revised Cardiac Risk Index vs. ASA Status as a Predictor for Non-Cardiac Events Following Posterior Lumbar Decompression. World Neurosurgery 2018, 12:12.

14. Brunelli A, Varela G, Salati M, Jimenez MF, Pompili C, Novoa N, Sabbatini A: Recalibration of the Revised Cardiac Risk Index in Lung Resection Candidates. Annals of Thoracic Surgery 2010, 90(1):199-203.

15. Bryce GJ, Payne CJ, Gibson SC, Kingsmore DB, Byrne DS, Delles C: Risk Stratification Scores in Elective Open Abdominal Aortic Aneurysm Repair: Are They Suitable for Preoperative Decision Making? European Journal of Vascular and Endovascular Surgery 2012, 44(1):55-61.

16. Canbolat IP, Erdogan Y, Adali G, Kaplan O, Dayangac M, Yuzer Y, Tokat Y: The predictive value of risk indices for cardiac complications in living donor liver transplantation. Bratislava Medical Journal-Bratislavske Lekarske Listy 2018, 119(5):289-293.

17. Carabini LM, Zeeni C, Moreland NC, Gould RW, Hemmer LB, Bebawy JF, Koski TR, McClendon J, Jr., Koht A, Gupta DK: Predicting Major Adverse Cardiac Events in Spine Fusion Patients. Spine 2014, 39(17):1441-1448.

18. Che L, Xu L, Huang Y, Yu C: Clinical utility of the revised cardiac risk index in older Chinese patients with known coronary artery disease. Clinical Interventions in Aging 2018, 13:35-41.

19. Choi JH, Cho DK, Song YB, Hahn JY, Choi S, Gwon HC, Kim DK, Lee SH, Oh JK, Jeon ES: Preoperative NT-proBNP and CRP predict perioperative major cardiovascular events in noncardiac surgery. Heart 2010, 96(1):56-62.

20. Cohn SL, Fernandez Ros N: Comparison of 4 Cardiac Risk Calculators in Predicting Postoperative Cardiac Complications After Noncardiac Operations. American Journal of Cardiology 2018, 121(1):125-130.

21. Cuthbertson BH, Almiri AR, Croal BL, Rajagopalan S, Alozairi O, Brittenden J, Hillis GS: Utility of B-type natriuretic peptide in predicting perioperative cardiac events in patients undergoing major non-cardiac surgery. British Journal of Anaesthesia 2007, 99(2):170-176.

22. Datema FR, Poldermans D, de Jong RJB: INCIDENCE AND PREDICTION OF MAJOR CARDIOVASCULAR COMPLICATIONS IN HEAD AND NECK SURGERY. Head and Neck-Journal for the Sciences and Specialties of the Head and Neck 2010, 32(11):1485-1493.

23. Davis C, Tait G, Carroll J, Wijeysundera DN, Beattie WS: The Revised Cardiac Risk Index in the new millennium: a single-centre prospective cohort re-evaluation of the original variables in 9,519 consecutive elective surgical patients. Canadian Journal of Anesthesia-Journal Canadien D Anesthesie 2013, 60(9):855-863.

24. Dhillon AK, Disque AA, Nguyen-Buckley CT, Grogan TR, Russell DL, Gritsch HA, Neelankavil JP: Does A Low 6-Minute Walk Distance Predict Elevated Postoperative Troponin? Anesthesia and Analgesia 2018, 127(2):E1-E3.

25. Dillon JK, Liu SY, Patel CM, Schmidt BL: IDENTIFYING RISK FACTORS FOR POSTOPERATIVE CARDIOVASCULAR AND RESPIRATORY COMPLICATIONS AFTER MAJOR ORAL CANCER SURGERY. Head and Neck-Journal for the Sciences and Specialties of the Head and Neck2011, 33(1):112-116.

26. Ehlert BA, Najafian A, Orion KC, Malas MB, Black JH, III, Abularrage CJ:Validation of a modified Frailty Index to predict mortality in vascular surgery patients. Journal of Vascular Surgery 2016, 63(6):1595-+. 
27. Feringa HH, Vidakovic R, Karagiannis SE, de Jonge R, Lindemans J, Goei D, Schouten O, Bax $\mathrm{JJ}$, Poldermans D: Baseline natriuretic peptide levels in relation to myocardial ischemia, troponin T release and heart rate variability in patients undergoing major vascular surgery. Coronary Artery Disease 2007, 18(8):645-651.

28. Fisher BW, Ramsay G, Majumdar SR, Hrazdil CT, Finegan BA, Padwal RS, McAlister FA: The ankle-to-arm blood pressure index predicts risk of cardiac complications after noncardiac surgery. Anesthesia and Analgesia 2008, 107(1):149-154.

29. Gillmann H-J, Meinders A, Grosshennig A, Larmann J, Buente C, Calmer S, Sahlmann B, Rustum S, Aper T, Lichtinghagen Ret al: Perioperative Levels and Changes of High-Sensitivity Troponin TAre Associated With Cardiovascular Events in Vascular Surgery Patients. Critical Care Medicine 2014, 42(6):1498-1506.

30. Golubovic M, Stanojevic D, Lazarevic M, Peric V, Kostic T, Djordjevic M, Zivic S, Milic DJ: A Risk Stratification Model for Cardiovascular Complications during the 3-Month Period after Major Elective Vascular Surgery. Biomed Research International 2018.

31. Gualandro DM, Puelacher C, LuratiBuse G, Llobet GB, Yu PC, Cardozo FA, Glarner N, Zimmerli A, Espinola J, Corbiere S et al: Prediction of major cardiac events after vascular surgery. Journal of Vascular Surgery 2017, 66(6):1826-+.

32. Gupta PK, Gupta H, Sundaram A, Kaushik M, Fang X, Miller WJ, Esterbrooks DJ, Hunter CB, Pipinos II, Johanning JM et al: Development and Validation of a Risk Calculator for Prediction of Cardiac Risk After Surgery. Circulation 2011, 124(4):381-U146.

33. Hwang J-w, Kim E-K, Yang J-H, Chang S-A, Bin Song Y, Hahn J-Y, Choi SH, Gwon H-C, Lee S-H, Kim S-M et al: Assessment of Perioperative Cardiac Risk of Patients Undergoing Noncardiac Surgery Using Coronary Computed Tomographic Angiography. Circulation-Cardiovascular Imaging 2015, 8(3).

34. James S, Jhanji S, Smith A, O’Brien G, Fitzgibbon M, Pearse RM: Comparison of the prognostic accuracy of scoring systems, cardiopulmonary exercise testing, and plasma biomarkers: a single-centre observational pilot study. British Journal of Anaesthesia 2014, 112(3):491-497.

35. Jarai R, Mahla E, Perkmann T, Jarai R, Archan S, Tentzeris I, Huber K, Metzler H: Usefulness of Pre-Operative Copeptin Concentrations to Predict Post-Operative Outcome After Major Vascular Surgery. American Journal of Cardiology 2011, 108(8):1188-1195.

36. Karkos CD, Thomson GJL, Hughes R, Hollis S, Hill JC, Mukhopadhyay US: Prediction of cardiac risk before abdominal aortic reconstruction: Comparison of a revised Goldman Cardiac Risk Index and radioisotope ejection fraction. Journal of Vascular Surgery 2002, 35(5):943-949.

37. Katsanos S, Babalis D, Kafkas N, Mavrogenis A, Leong D, Parissis J, Varounis C, Makris K, van der Heijden A, Anastasiou-Nana M et al: B-type natriuretic peptide vs. cardiac risk scores for prediction of outcome following major orthopedic surgery. Journal of Cardiovascular Medicine 2015, 16(6):465-471.

38. Kaw R, Nagarajan V, Jaikumar L, Halkar M, Mohananey D, Hernandez AV, Ramakrishna H, Wijeysundera D: Predictive Value of Stress Testing, Revised Cardiac Risk Index, and Functional Status in Patients Undergoing Non Cardiac Surgery. Journal of Cardiothoracic \& Vascular Anesthesia 2018, 24:24.

39. Kertai MD, Boersma E, Klein J, van Urk H, Poldermans D: Optimizing the prediction of perioperative mortality in vascular surgery by using a customized probability model. Archives of Internal Medicine 2005, 165(8):898-904. 
40. Kopec M, Duma A, Helwani MA, Brown J, Brown F, Gage BF, Gibson DW, Miller JP, Novak E, Jaffe AS et al: Improving Prediction of Postoperative Myocardial Infarction With High-Sensitivity Cardiac Troponin T and NT-proBNP. Anesthesia and Analgesia 2017, 124(2):398-405.

41. Leibowitz D, Planer D, Rott D, Elitzur Y, Chajek-Shaul T, Weiss AT: Brain natriuretic peptide levels predict perioperative events in cardiac patients undergoing noncardiac surgery: A prospective study. Cardiology 2008, 110(4):266-270.

42. Makary MA, Segev DL, Pronovost PJ, Syin D, Bandeen-Roche K, Patel P, Takenaga R, Devgan L, Holzmueller CG, Tian J et al: Frailty as a Predictor of Surgical Outcomes in Older Patients. Journal of the American College of Surgeons 2010, $210(6): 901-908$.

43. Markovic D, Jevtovic-Stoimenov T, Cosic V, Stosic B, Dinic V, Markovic-Zivkovic B, Jankovic RJ: CLINICAL UTILITY OF SURVIVIN (BIRC5), NOVEL CARDIAC BIOMARKER, AS A PROGNOSTIC TOOL COMPARED TO HIGH-SENSITIVITY C-REACTIVE PROTEIN, HEART-TYPE FATTY ACID BINDING PROTEIN AND REVISED LEE SCORE IN ELDERLY PATIENTS SCHEDULED FOR MAJOR NON-CARDIAC SURGERY: A PROSPECTIVE PILOT STUDY. Journal of Medical Biochemistry 2018, 37(2):110-120.

44. Markovic D, Jevtovic-Stoimenov T, Stojanovic M, Vukovic A, Dinic V, Markovic-Zivkovic B, Jankovic RJ: Addition of clinical risk scores improves prediction performance of American Society of Anesthesiologists (ASA) physical status classification for postoperative mortality in older patients: a pilot study. European Geriatric Medicine 2018, 9(1):51-59.

45. Mauermann E, Bolliger D, Seeberger E, Puelacher C, Corbiere S, Filipovic M, Seeberger M, Mueller C, Buse GL: Incremental Value of Preoperative Copeptin for Predicting Myocardial Injury. Anesthesia and Analgesia 2016, 123(6):1363-1371.

46. McAlister FA, Jacka M, Graham M, Youngson E, Cembrowski G, Bagshaw SM, Pannu N, Townsend DR, Srinathan S, Alonso-Coello P et al: The prediction of postoperative stroke or death in patients with preoperative atrial fibrillation undergoing non-cardiac surgery: a VISION sub-study. Journal of Thrombosis and Haemostasis 2015, 13(10):1768-1775.

47. Mcllroy DR, Chan MTV, Wallace SK, Symons JA, Koo EGY, Chu LCY, Myles PS: Automated preoperative assessment of endothelial dysfunction and risk stratification for perioperative myocardial injury in patients undergoing non-cardiac surgery. British Journal of Anaesthesia 2014, 112(1):47-56.

48. Mercantini P, Di Somma S, Magrini L, Nava AK, Scarinci A, La Torre M, Ferri M, Ferri E, Petrucciani N, Ziparo V: Preoperative Brain Natriuretic Peptide (BNP) Is a Better Predictor of Adverse Cardiac Events Compared to Preoperative Scoring System in Patients Who Underwent Abdominal Surgery. World Journal of Surgery 2012, 36(1):24-30.

49. Moodley Y, Naidoo P, Biccard BM: The South African Vascular Surgical Cardiac Risk Index (SAVS-CRI): A prospective observational study. Samj South African Medical Journal 2013, 103(10):746-750.

50. Neary WD, Prytherch D, Foy C, Heather BP, Earnshaw JJ: Comparison of different methods of risk stratification in urgent and emergency surgery. British Journal of Surgery 2007, 94(10):1300-1305.

51. Noordzij PG, Boersma E, Bax JJ, Feringa HHH, Schreiner F, Schouten O, Kertai MD, Klein J, van Urk H, Elhendy A et al: Prognostic value of routine preoperative electrocardiography in patients undergoing noncardiac surgery. American Journal of Cardiology 2006, 97(7):11031106. 
52. Pandey A, Sood A, Sammon JD, Abdollah F, Gupta E, Golwala H, Bardia A, Kibel AS, Menon M, Trinh Q-D: Effect of Preoperative Angina Pectoris on Cardiac Outcomes in Patients With Previous Myocardial Infarction Undergoing Major Noncardiac Surgery (Data from ACSNSQIP). American Journal of Cardiology 2015, 115(8):1080-1084.

53. Pantoja Muñoz HJ, Fernández Ramos H, Guevara Tovar WL: Sensitivity, specificity and predictive values of the Goldman, Detsky and Lee cardiac indices. Revista Colombiana de Anestesiologia 2014, 42(3):184-191.

54. Park S-J, Choi J-H, Cho S-J, Chang S-A, Choi J-O, Lee S-C, Park SW, Oh JK, Kim D-K, Jeon E-S: Comparison of Transthoracic Echocardiography With N-Terminal Pro-Brain Natriuretic Peptide as a Tool for Risk Stratification of Patients Undergoing Major Noncardiac Surgery. Korean Circulation Journal 2011, 41(9):505-511.

55. Parmar CD, Torella F: Prediction of Major Adverse Cardiac Events in Vascular Surgery: Are Cardiac Risk Scores of Any Practical Value? Vascular and Endovascular Surgery 2010, 44(1):1419.

56. Peterson B, Ghahramani M, Harris S, Suchniak-Mussari K, Bedi G, Bulathsinghala C, Foy A: Usefulness of the Myocardial Infarction and Cardiac Arrest Calculator as a Discriminator of Adverse Cardiac Events After Elective Hip and Knee Surgery. American Journal of Cardiology 2016, 117(12):1992-1995.

57. Press MJ, Chassin MR, Wang J, Tuhrim S, Halm EA: Predicting medical and surgical complications of carotid endarterectomy - Comparing the risk indexes. Archives of Internal Medicine 2006, 166(8):914-920.

58. Ray MJ, Calabro LJ, Sirisena T, Crawford SA, Crawford RW, Walters DL: Pre-operative plateletbound CD40 ligand is probably associated with peri-operative cardiac events in hip and knee arthroplasty. European Journal of Clinical Investigation 2010, 40(6):497-503.

59. Rodseth RN, Buse GAL, Bolliger D, Burkhart CS, Cuthbertson BH, Gibson SC, Mahla E, Leibowitz DW, Biccard BM: The Predictive Ability of Pre-Operative B-Type Natriuretic Peptide in Vascular Patients for Major Adverse Cardiac Events An Individual Patient Data Meta-Analysis. Journal of the American College of Cardiology 2011, 58(5):522-529.

60. Rohde LE, Polanczyk CA, Goldman L, Cook EF, Lee RT, Lee TH: Usefulness of transthoracic echocardiography as a tool for risk stratification of patients undergoing major noncardiac surgery. American Journal of Cardiology 2001, 87(5):505-509.

61. Rohrig R, Junger A, Hartmann B, Klasen J, Quinzio L, Jost A, Benson M, Hempelmann G: The incidence and prediction of automatically detected intraoperative cardiovascular events in noncardiac surgery. Anesthesia and Analgesia 2004, 98(3):569-577.

62. Sabate S, Mases A, Guilera N, Canet J, Castillo J, Orrego C, Sabate A, Fita G, Parramon F, Paniagua P et al: Incidence and predictors of major perioperative adverse cardiac and cerebrovascular events in non-cardiac surgery. British Journal of Anaesthesia 2011, 107(6):879890.

63. Saito S, Takagi A, Kurokawa F, Ashihara K, Hagiwara N: Usefulness of tissue Doppler echocardiography to predict perioperative cardiac events in patients undergoing noncardiac surgery. Heart and Vessels 2012, 27(6):594-602.

64. Schouten O, Kok NFM, Hoedt MTC, van Laanen JHH, Poldermans D: The influence of aneurysm size on perioperative cardiac outcome in elective open infrarenal aortic aneurysm repair. Journal of Vascular Surgery 2006, 44(3):435-441. 
65. Schrimpf C, Gillmann H-J, Sahlmann B, Meinders A, Larmann J, Wilhelmi M, Aper T, Rustum S, Lichtinghagen $\mathrm{R}$, Theilmeier $\mathrm{G}$ et al: Renal function interferes with copeptin in prediction of major adverse cardiac events in patients undergoing vascular surgery. PLOS ONE [Electronic Resource] 2015, 10(4):e0123093.

66. Scrutinio D, Guido G, Guida P, Passantino A, Angiletta D, Santoro D, Marinazzo D, Regina G: Combined Use of High-sensitivity C-Reactive Protein and N-Terminal Pro-B-type Natriuretic Peptide for Risk Stratification of Vascular Surgery Patients. Annals of Vascular Surgery 2014, 28(6):1522-1529.

67. Sheth T, Chan M, Butler C, Chow B, Tandon V, Nagele P, Mitha A, Mrkobrada M, Szczeklik W, Faridah Y et al: Prognostic capabilities of coronary computed tomographic angiography before non-cardiac surgery: prospective cohort study. BMJ-British Medical Journal 2015, 350.

68. Avena Smeili LA, Lotufo PA: Incidence and Predictors of Cardiovascular Complications and Death after Vascular Surgery. Arquivos Brasileiros De Cardiologia 2015, 105(5):510-518.

69. Stonelake S, Thomson P, Suggett N: Identification of the high risk emergency surgical patient: Which risk prediction model should be used? Annals of Medicine and Surgery 2015, 4(3):240247.

70. Subramaniam B, Meroz Y, Talmor D, Pomposelli FB, Berlatzky Y, Landesberg G: A LongTerm Survival Score Improves Preoperative Prediction of Survival Following Major Vascular Surgery. Annals of Vascular Surgery 2011, 25(2):197-203.

71. Valentijn TM, Hoeks SE, Bakker EJ, Voute MT, Chonchol M, van de Luijtgaarden KM, Verhagen HJ, Stolker RJ: Influence of Aortic Valve Calcium on Outcome in Patients Undergoing Peripheral Vascular Surgery. American Journal of Cardiology 2012, 110(8):1195-1199.

72. van Diepen S, Youngson E, Ezekowitz JA, McAlister FA: Which risk score best predicts perioperative outcomes in nonvalvular atrial fibrillation patients undergoing noncardiac surgery? American Heart Journal 2014, 168(1):60-67.

73. van Klei WA, Bryson GL, Yang H, Kalkman CJ, Wells GA, Beattie WS: The value of routine preoperative electrocardiography in predicting myocardial infarction after noncardiac surgery. Annals of Surgery 2007, 246(2):165-170.

74. Vetrugno L, Langiano N, Gisonni R, Rizzardo A, Venchiarutti PE, Divella M, Pompei L, Causero A, Della Rocca G: Prediction of early postoperative major cardiac events after elective orthopedic surgery: the role of B-type natriuretic peptide, the revised cardiac risk index, and ASA class. Bmc Anesthesiology 2014, 14.

75. Vilarino-Rico J, Pita-Fernandez S, Joaquin Segura-Iglesias R: Clinical Predictors of Major Adverse Cardiovascular Events during Long-Term Follow-Up after Carotid Endarterectomy. Annals of Vascular Surgery 2015, 29(3).

76. Waterman BR, Belmont PJ, Jr., Bader JO, Schoenfeld AJ: The Total Joint Arthroplasty Cardiac Risk Index for Predicting Perioperative Myocardial Infarction and Cardiac Arrest After Primary Total Knee and Hip Arthroplasty. Journal of Arthroplasty 2016, 31(6):1170-1174.

77. Weber M, Luchner A, Manfred S, Mueller C, Liebetrau C, Schlitt A, Apostolovic S, Jankovic R, Bankovic D, Jovic M et al: Incremental value of high-sensitive troponin T in addition to the revised cardiac index for peri-operative risk stratification in non-cardiac surgery. European Heart Journal 2013, 34(11):853-862. 
78. Welten GMJM, Schouten O, van Domburg RT, Feringa HHH, Hoeks SE, Dunkelgrun M, van Gestel YRBM, Goei D, Bax JJ, Poldermans D: The influence of aging on the prognostic value of the revised cardiac risk index for postoperative cardiac complications in vascular surgery patients. European Journal of Vascular and Endovascular Surgery 2007, 34(6):632-638.

79. Wijeysundera DN, Pearse RM, Shulman MA, Abbott TEF, Torres E, Ambosta A, Croal BL, Granton JT, Thorpe KE, Grocott MPW et al: Assessment of functional capacity before major non-cardiac surgery: an international, prospective cohort study. Lancet 2018, 391(10140):2631-2640.

80. Wotton R, Marshall A, Kerr A, Bishay E, Kalkat M, Rajesh P, Steyn R, Naidu B: Does the revised cardiac risk index predict cardiac complications following elective lung resection? Journal of Cardiothoracic Surgery 2013, 8.

81. Yang HS, Hur M, Yi A, Kim H, Kim J: Prognostic Role of High-sensitivity Cardiac Troponin I and Soluble Suppression of Tumorigenicity-2 in Surgical Intensive Care Unit Patients Undergoing Non-cardiac Surgery. Annals of Laboratory Medicine 2018, 38(3):204-211.

82. Yang JH, Choi JH, Ki Y-W, Kim DI, Kim D-K, Park JR, Oh JK, Choi S-H: Plasma N-Terminal Pro-BType Natriuretic Peptide Is Predictive of Perioperative Cardiac Events in Patients Undergoing Vascular Surgery. Korean Journal of Internal Medicine 2012, 27(3):301-310.

83. Yap MKC, Ang KF, Gonzales-Porciuncula LA, Esposo E: Validation of the American College of Surgeons Risk Calculator for preoperative risk stratification. Heart Asia 2018, 10 (2):e010993. 


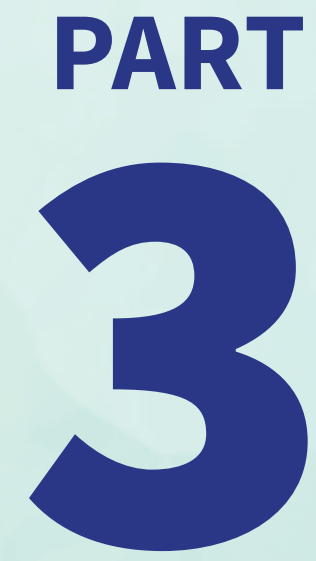

\section{TROPONIN ELEVATION AFTER MAJOR NON-CARDIAC SURGERY}




\section{CHAPTER}

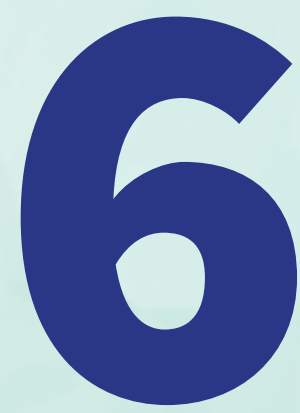

Postoperative visits by dedicated anaesthesiologists in patients with elevated troponin: a retrospective cohort study evaluating postoperative care utility and early detection of complications

Lisette M. Vernooij* Annemarie Akkermans*

Wilton A. van Klei Judith A.R. van Waes * Authors contributed equally 


\section{ABSTRACT}

Background: An elevated cardiac troponin level after non-cardiac surgery is associated with both morbidity and mortality. Guidelines suggest routine troponin monitoring in high-risk patients. We implemented a dedicated anaesthesia team to conduct follow-up on patients with postoperative troponin elevation. We hypothesized that these visits would facilitate early detection of complications. Therefore, the aim of this study was to evaluate the effect of postoperative visits by dedicated anaesthesiologists on early detection of complications and care utility.

Methods: This retrospective observational study included patients aged ${ }^{3} 60$ years with an elevated troponin within the first three days after non-cardiac surgery. Troponin elevation was detected by routine biomarker monitoring. The primary outcome was early detected myocardial infarctions by the dedicated anaesthesiologist. Other outcomes were overall detected complications, additional diagnostic tests and treatment advised by the anaesthesiologist, consultation of another medical specialist, and advised postoperative follow-up at the outpatient cardiac clinic within one week after surgery.

Results: Of the 811 patients, 509 (63\%) received a postoperative consultation by the anaesthesiologist. Anesthesiologists were involved in the early detection of $59 \%$ of all myocardial infarctions and in $12 \%$ of all complications. Besides cardiac ischemia, patients were also often diagnosed with non-cardiac complications, including respiratory failure (8.9\%), pneumonia (13.2\%) and acute kidney injury (17.5\%) within one week after surgery. In 75\% of patients, anaesthesiologists ordered additional diagnostics, most frequently existing of electrocardiograms and additional cardiac enzyme testing. Additionally, change in treatment was advised, most often a medication change, in $16 \%$ of patients.

Conclusions: Standard consultation of a dedicated anaesthesiologist resulted in an early detection of $59 \%$ of all myocardial infarctions and involved a change in treatment in a considerable number of patients with postoperative troponin elevation. Whether this may improve patient outcomes remains to be elucidated.

Keywords: troponin, perioperative anaesthesiologist, screening, myocardial infarction 


\section{INTRODUCTION}

An increased serum cardiac troponin level, as a marker for postoperative myocardial injury, has been shown to be an independent predictor of morbidity and mortality within the first year after non-cardiac surgery. (1-4) Depending on the amount of troponin elevation and the surgical population, the incidence of mortality within 30 days varies between $4 \%$ and $17 \%$. (1, 4 , 5) As only $15 \%$ of patients with postoperative myocardial injury experiences typical ischemic symptoms, (6) routine postoperative troponin surveillance is recommended by several guidelines for postoperative risk stratification to direct secondary prevention. $(7,8)$

In our centre, we implemented a routine postoperative troponin I (TnI) surveillance program in January 2011. In a previous study in which this program was evaluated, it became apparent that only $41 \%$ of all patients with postoperative myocardial injury received cardiac consultation. (9) Cardiac ethology was suspected in almost half of the consulted patients. However, troponin elevation has also been related to other disease entities including stroke, sepsis and pulmonary embolism. (3, 10-12) We hypothesized a more general approach to assess postoperative troponin elevation, rather than an approach focusing on cardiac ethology, would be beneficial. As a result, in October 2016 we implemented routine postoperative consultations by a dedicated anaesthesia team in patients detected with an elevated troponin in the surveillance program. The goal of these visits was to improve postoperative follow-up and to potentially detect or prevent complications at an early stage. We hypothesized that such routine visits would facilitate early detection of complications with only a limited use of resources. Therefore, this study aimed to evaluate the effect of these visits by dedicated anaesthesiologists on early detection of complications and postoperative care utility in patients with troponin elevation after non-cardiac surgery.

\section{METHODS}

This study was conducted in adherence to the STROBE statement for observational research. (13) The local Research Ethics Committee assessed the study protocol and waived the need for informed consent (UMC Utrecht Medical Research Ethics Committee 19-029/C).

\section{Patients}

This retrospective observational study included patients aged ${ }^{3} 60$ years with postoperative elevated troponin levels as detected by routine troponin monitoring within the first three days after non-cardiac surgery between January $1^{\text {st }}, 2017$ and December 31 $31^{\text {th }}, 2018$ at the University Medical Center Utrecht, a tertiary referral hospital. Patients admitted immediately after the procedure at the Intensive Care Unit (ICU) or the Cardiac Care Unit (CCU) for more than two days were not visited by the dedicated anaesthesiologists and were therefore excluded from the analysis, because follow-up in these patients was conducted by intensivists or cardiologists as part of our local protocol. Patients who died within 24 hours or in whom further therapy was 
withheld directly after surgery were also excluded. Patients, who underwent surgery more than once within the study period, were included as a new case. However, in case the procedure was performed within three days after the previous procedure, only the first procedure was included in the analysis.

\section{Postoperative care}

According to our local postoperative care protocol, cardiac troponin I (Tnl) is measured in all noncardiac surgical patients aged ${ }^{3} 60$ years once daily on the first three postoperative days during hospital admission. This protocol excludes ophthalmic and plastic surgery patients because of low risk of cardiac complications. Troponin elevation was defined as Tnl above the clinical cutoff level, which is the lowest value measurable with a $10 \%$ coefficient of variation above the 99 th percentile upper reference limit. (8) Two different Tnl assays were used over the years. This resulted in a clinical cut-off of Tnl $\geq 60 \mathrm{ng} \mathrm{L}^{-1}$ (AccuTnl assay, Beckman Coulter, Brea, California, USA) from January 1 $1^{\text {st }}, 2017$ until May 16 th $^{\text {th }}, 2018$ and a clinical cut-off of high-sensitive Tnl $\geq 18 \mathrm{ng} \mathrm{L}^{-1}$ (Unicel Dxl 800, Beckman Coulter, Brea, California, USA) from May 17 $7^{\text {th }}, 2018$ until December 31 $1^{\text {st }}, 2018$.

Patients with elevated Tnl were consulted within the first three postoperative days, and longer if indicated. Management of these patients was left to the judgement of the attending anaesthesiologist, which was based on a local protocol. This protocol advises to optimize myocardial oxygen supply and demand, and to conduct follow-up of troponin and an electrocardiogram (ECG) in patients with $\mathrm{Tnl}>120 \mathrm{ng} \mathrm{L}^{-1}$ in case of no evident non-ischemic cause (e.g. sepsis, stress induced cardiomyopathy) to rule out myocardial ischemia. This cut-off was based on two times the clinical cut-off of the assay used at that moment. Further, this protocol advises to consider consultation of a cardiologist, follow-up at the outpatient cardiac clinic, and prescription of antiplatelet therapy, statin or beta-blockade. In patients with troponin >600 ng $\mathrm{L}^{-1}$, i.e. more than ten times the clinical cut-off, a cardiology consultation is always recommended. Patients with mild troponin elevation were per protocol frequently not visited physically by the anaesthesiologist because of a low risk of serious complications, but were only followed up by $\mathrm{Tnl}$ and ECG. A team of ten dedicated anaesthesiologists with a particular interest in perioperative medicine were educated on the protocol.

\section{Data collection}

All data were collected from electronic medical files. These data included patient characteristics, comorbidities, revised cardiac risk index (RCRI), (14) American Society of Anesthesiologists (ASA) physical status classification (15), Metabolic Equivalent Task score (METs), (16) and surgical risk as defined by the RCRI, and by the European Society of Cardiology (ESC) and the European Society of Anaesthesiology (ESA). (16) Additionally, data on visits by the dedicated anaesthesiologist, 
diagnostics, in-hospital postoperative complications, severity of complications according to the Clavien-Dindo classification, $(17,18)$ and in-hospital mortality were collected.

\section{Outcome}

The primary outcome was early detected (i.e. $\leq 7$ days after surgery) myocardial infarction as a result of consultation by the dedicated anaesthesiologist. Myocardial infarction was defined as clinically diagnosed by the attending cardiologist. Secondary outcomes were overall detected complications, additional diagnostic tests advised by the dedicated anaesthesiologist, treatment advised by the anaesthesiologist, consultation of another medical specialist, and advised postoperative follow-up at the outpatient cardiac clinic. Potentially, a cardiologist could already have been consulted by the ward physician in patients with an elevated troponin or other cardiac complications, as the ward physicians were aware of the protocol. Therefore, we also recorded whether a cardiologist was consulted prior to or simultaneously with the visit by the dedicated anaesthesiologists.

In addition to myocardial infarction, we assessed the occurrence of the following complications within seven days after the procedure: arrhythmia diagnosed on 12-leads ECG or cardiac monitor, cardiopulmonary resuscitation, cerebrovascular accident (defined as radiologically-confirmed ischemic or haemorrhagic stroke or transient ischemic attack), radiologically-confirmed deep venous thrombosis and pulmonary embolism, sepsis as clinically diagnosed by the treating physician, pneumonia requiring antibiotics, respiratory failure requiring MCU (Medium Care Unit) or ICU admission, acute kidney injury (AKI) defined as an increase in creatinine of $26.4 \mathrm{mmol} \mathrm{L}^{-1}$ or 25\% from the preoperative creatinine value, (18) anaemia defined as haemoglobin $<6.0 \mathrm{mmol}$ $\mathrm{L}^{-1}$ (10 $\mathrm{g} \mathrm{dL}^{-1}$, according to the Dutch guideline on blood transfusion, (19) unexpected MCU or ICU admission, and unplanned reoperations. Additionally, length of hospital stay, mortality within seven days and the cause of death were assessed. Last, the Clavien-Dindo grade of the most severe complication within a week after surgery was recorded. A severe complication was defined as a Clavien-Dindo grade $\geq 3$ as this involves complications requiring a surgical, endoscopic or radiologic intervention, life threatening complications requiring care in a high-dependency or intensive care unit, or death. $(17,18)$ Additionally, we assessed the final suspected ethology of the elevated troponin as proposed by the dedicated anaesthesiologist.

\section{Statistical analyses}

Baseline characteristics were compared dependent on the amount of troponin elevation (i.e. Tnl 18-119 $\mathrm{ng} \mathrm{L}^{-1}$, Tnl 120-599 $\mathrm{ng} \mathrm{L}^{-1}$ and $\left.\mathrm{Tnl} \geq 600 \mathrm{ng} \mathrm{L}^{-1}\right)$. These thresholds were chosen based on the thresholds as defined in our local protocol. The contribution of the dedicated anaesthesiologist on the early detection of complications was assessed relative to the total number of complications. In addition, complications by their severity, length of stay, unexpected ICU or MCU admission and 
death were assessed. The consultation rates by the dedicated anaesthesiologists were evaluated, as were the number of ordered diagnostics, consulted medical specialties and advised therapies. In order to determine whether care utility and complications were dependent on the height of troponin elevation, these were evaluated in subgroups of patients with different levels of troponin (i.e. Tnl 18-119 ng L L-1 , Tnl 120-599 $n g \mathrm{~L}^{-1}$ and $T n l \geq 600 \mathrm{ng} \mathrm{L}^{-1}$ ).

Haemoglobin and creatinine measurements were missing in 103 and 204 patients, respectively. Since these variables were only used for descriptive statistics, we did not consider this an important source of bias and we did not impute the data. The statistical analyses were performed with R (Version 3.5.1 (c) 2018-07-02, R, Inc., for Windows).

\section{RESULTS}

Within the study period, 8924 patients underwent noncardiac surgery of whom 1007 patients (11.2 $\%)$ had troponin elevation. Of these patients, 811 were eligible for inclusion (Figure 1). Reason of exclusion was most often due to ICU admission for $>2$ days ( $n=122$ ). Troponin was mildly elevated, i.e. 18-119 ng L-1 in 543 patients (67\%), moderately elevated, i.e. 120-600 ng L-1 in 192 patients (24\%) and highly elevated, i.e. $\geq 600 \mathrm{ng}$ L-1 in 77 patients (9\%).

Baseline characteristics are reported in Table 1. The median age was 74 years [IQR 68-80] and $62 \%$ of the patients were males. Most patients underwent general (28\%) or vascular (24\%) surgery and surgery was emergent in $40 \%$ of the patients. There were more patients with a history of ischemic heart disease and peripheral vascular disease in the group with highly elevated troponin compared to the other two categories.

\section{Postoperative outcomes}

A total of 804 complications occurred in 462 patients within seven days after surgery (Table 2). The involvement of the dedicated anesthesiologist led to the diagnosis of 97 (12\%) of these complications in 63 patients (13\%). Postoperative complications were identified by the anesthesiologist in 18 patients (5\%) with a Tnl 18-119 ng L-1, 21 patients (13\%) with a Tnl 120-599 ng L-1 and 24 patients (33\%) with a Tnl $\geq 600$ ng L-1. The anesthesiologists were involved in the discovery of 19 (59\%) of 32 postoperative myocardial infarctions within one week after surgery. Besides cardiac ischemia, 76 (9.4\%) patients developed an arrhythmia, but patients were also often diagnosed with noncardiac complications, including respiratory failure (8.9\%), pneumonia (13.2\%) and acute kidney injury (17.5\%). Patients with higher troponin levels suffered from more complications, had more often an unexpected ICU or MCU admission and had a longer median hospital length of stay. Ten patients (1\%) died in the first week after surgery and 251 patients (31\%) had a complication graded as Clavien-Dindo $\geq 3$ (Table 2). 
Figure 1. Patient flow chart

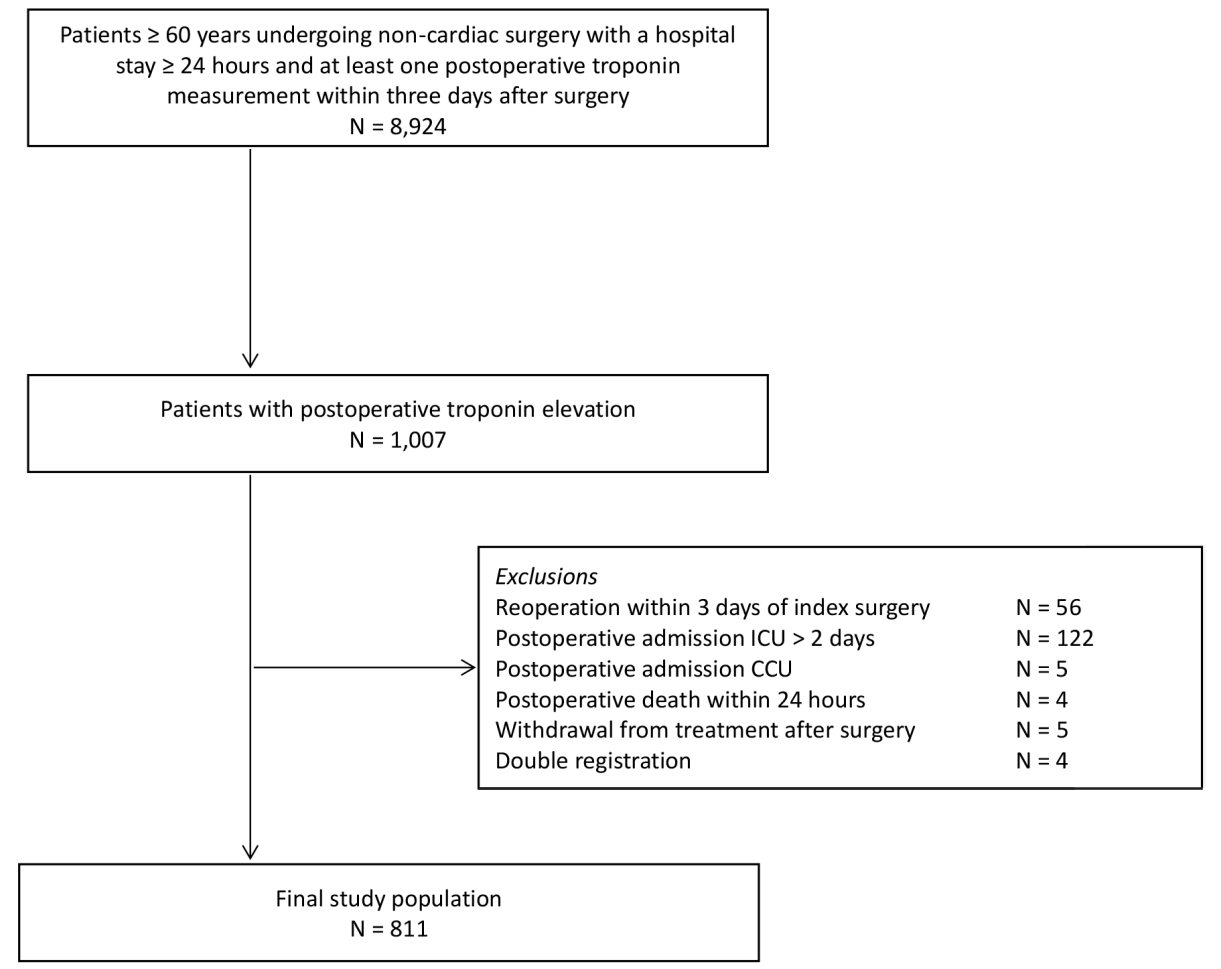

Table 1. Baseline characteristics dependent on the height of troponin elevation

\begin{tabular}{|c|c|c|c|c|}
\hline & Overall & $\begin{array}{c}\text { Tnl 18-119 } \\
n g ~ L^{-1}\end{array}$ & $\begin{array}{c}\text { Tnl } \\
120-599 \mathrm{ng} \mathrm{L}^{-1}\end{array}$ & $\begin{array}{c}\mathrm{Tnl} \geq 600 \\
n g \mathrm{~L}^{-1}\end{array}$ \\
\hline$n$ & 811 & 543 & 192 & 76 \\
\hline Age (median [IQR]) & $74[68-80]$ & $74[68-80]$ & $74[68-79]$ & $73[69-79]$ \\
\hline Males (\%) & $505(62.3)$ & $341(62.8)$ & $115(59.9)$ & $49(64.5)$ \\
\hline \multicolumn{5}{|l|}{ RCRI score (\%) } \\
\hline 0 & $196(24.2)$ & $141(26.0)$ & $45(23.4)$ & $10(13.2)$ \\
\hline 1 & $320(39.5)$ & $215(39.6)$ & $77(40.1)$ & $28(36.8)$ \\
\hline 2 & $191(23.6)$ & $120(22.1)$ & $45(23.4)$ & $26(34.2)$ \\
\hline 3 or more & $104(12.8)$ & $67(12.3)$ & $25(13.0)$ & $12(15.8)$ \\
\hline High-risk surgery (defined by RCRI) (\%) & $314(38.7)$ & $195(35.9)$ & $82(42.7)$ & $37(48.7)$ \\
\hline High-risk surgery (defined by ESC/ESA) (\%) & $124(15.3)$ & $77(14.2)$ & $35(18.2)$ & $12(15.8)$ \\
\hline History of ischemic heart disease (\%) & $235(29.0)$ & $145(26.7)$ & $58(30.2)$ & $32(42.1)$ \\
\hline History of congestive heart failure $(\%)$ & $84(10.4)$ & $60(11.0)$ & $19(9.9)$ & $5(6.6)$ \\
\hline
\end{tabular}


Table 1. Baseline characteristics dependent on the height of troponin elevation

\begin{tabular}{|c|c|c|c|c|}
\hline & Overall & $\begin{array}{c}\text { Tnl 18-119 } \\
\mathrm{ng} \mathrm{L}^{-1}\end{array}$ & $\begin{array}{c}\text { Tnl } \\
120-599 \mathrm{ng} \mathrm{L}^{-1}\end{array}$ & $\begin{array}{c}\mathrm{Tn} \mathrm{I} \geq 600 \\
\mathrm{ng} \mathrm{L}^{-1}\end{array}$ \\
\hline History of cerebrovascular disease (\%) & $201(24.8)$ & $132(24.3)$ & $48(25.0)$ & $21(27.6)$ \\
\hline Insulin dependent diabetes (\%) & $109(13.4)$ & $74(13.6)$ & $24(12.5)$ & $11(14.5)$ \\
\hline Preoperative creatinine $>2.0 \mathrm{mg} \mathrm{dL}^{-1}(\%)$ & $113(13.9)$ & $83(15.3)$ & $18(9.4)$ & $12(15.8)$ \\
\hline Arrhythmia (\%) & $185(22.8)$ & $136(25.0)$ & $42(21.9)$ & $7(9.2)$ \\
\hline ICD or pacemaker (\%) & $65(8.0)$ & $53(9.8)$ & $10(5.2)$ & $2(2.6)$ \\
\hline Valvar disease (\%) & $113(13.9)$ & $82(15.1)$ & $23(12.0)$ & $8(10.5)$ \\
\hline Peripheral vascular disease (\%) & $241(29.7)$ & $140(25.8)$ & $66(34.4)$ & $35(46.1)$ \\
\hline Hypertension (\%) & $516(63.6)$ & $347(63.9)$ & $119(62.0)$ & $50(65.8)$ \\
\hline Pulmonary disease (\%) & $210(25.9)$ & $142(26.2)$ & $54(28.1)$ & $14(18.4)$ \\
\hline Active malignancy (\%) & $255(31.4)$ & $172(31.7)$ & $60(31.2)$ & $23(30.3)$ \\
\hline Renal failure (\%) & $282(34.8)$ & $194(35.7)$ & $58(30.2)$ & $30(39.5)$ \\
\hline Diabetes mellitus (\%) & $189(23.3)$ & $129(23.8)$ & $41(21.4)$ & $19(25.0)$ \\
\hline \multicolumn{5}{|l|}{ ASA physical status (\%) } \\
\hline 1 & $4(0.5)$ & $3(0.6)$ & $0(0.0)$ & $1(1.3)$ \\
\hline 2 & $229(28.2)$ & $161(29.7)$ & $53(27.6)$ & $15(19.7)$ \\
\hline 3 & $481(59.3)$ & $319(58.7)$ & $112(58.3)$ & $50(65.8)$ \\
\hline 4 & $88(10.9)$ & $55(10.1)$ & $25(13.0)$ & $8(10.5)$ \\
\hline 5 & $9(1.1)$ & $5(0.9)$ & $2(1.0)$ & $2(2.6)$ \\
\hline \multicolumn{5}{|l|}{ METS $(\%)$} \\
\hline 1-3 METS & $224(27.6)$ & $162(29.8)$ & $42(21.9)$ & $20(26.3)$ \\
\hline 4-7 METS & $373(46.0)$ & $263(48.4)$ & $79(41.1)$ & $31(40.8)$ \\
\hline 8-10 METS & $21(2.6)$ & $10(1.8)$ & $9(4.7)$ & $2(2.6)$ \\
\hline Unknown & $193(23.8)$ & $108(19.9)$ & $62(32.3)$ & $23(30.3)$ \\
\hline Emergency surgery (\%) & $328(40.4)$ & $213(39.2)$ & $83(43.2)$ & $32(42.1)$ \\
\hline \multicolumn{5}{|l|}{ Surgical specialty (\%) } \\
\hline General & $223(27.5)$ & $149(27.4)$ & $58(30.2)$ & $16(21.1)$ \\
\hline Gynaecological & $25(3.1)$ & $18(3.3)$ & $5(2.6)$ & $2(2.6)$ \\
\hline Head and Neck & $78(9.6)$ & $54(9.9)$ & $15(7.8)$ & $9(11.8)$ \\
\hline Neurosurgery & $133(16.4)$ & 94 (17.3) & $28(14.6)$ & $11(14.5)$ \\
\hline Orthopaedic & $104(12.8)$ & $71(13.1)$ & $26(13.5)$ & $7(9.2)$ \\
\hline Urological & $57(7.0)$ & $42(7.7)$ & $9(4.7)$ & $6(7.9)$ \\
\hline Vascular & $191(23.6)$ & $115(21.2)$ & $51(26.6)$ & $25(32.9)$ \\
\hline Locoregional \& neuraxial anaesthesia (\%) & $62(7.6)$ & $45(8.3)$ & $13(6.8)$ & $4(5.3)$ \\
\hline
\end{tabular}

ASA: American Society of Anesthesiologists classification. ESA: European Society of Anaesthesiology. ESC: European Society of Cardiology. ICD: implantable cardioverter defibrillator. IQR: interquartile range. METS: metabolic equivalent of task score. RCRI: revised cardiac risk index. Tnl: troponin I. Troponin thresholds were chosen based on the thresholds as defined in the local protocol of the University Medical Center Utrecht ( TnI 18-119 $\mathrm{ng} \mathrm{L}^{-1}$, Tnl 120-599 $\mathrm{Ng} \mathrm{L}^{-1}$ and $\mathrm{Tnl} \geq 600 \mathrm{ng} \mathrm{L}^{-1}$ ).

Ischemic heart disease was defined as a history of myocardial infarction or previous revascularization and a history of congestive heart failure was defined as an estimated left ventricular ejection fraction $<40 \%$. Cerebrovascular disease was defined as a history of ischemic stroke, haemorrhagic stroke or transient ischemic attacks. Renal failure was defined as a glomerular filtration rate (GFR) $<60 \mathrm{ml} \mathrm{min} \mathrm{m}^{-1}$ in the last three months). 
Table 2. Postoperative outcomes dependent on the height of troponin elevation

\begin{tabular}{|c|c|c|c|c|c|c|}
\hline & \multicolumn{2}{|c|}{$\begin{array}{l}\text { Tnl 18-119 } \\
\text { ng L L }^{-1}\end{array}$} & \multicolumn{2}{|c|}{$\begin{array}{c}\text { Tnl } \\
120-599 \mathrm{ng} \mathrm{L}^{-1}\end{array}$} & \multicolumn{2}{|c|}{$\begin{array}{c}\mathrm{TnI}^{3} \geq 600 \\
\mathrm{ng} \mathrm{L}^{-1}\end{array}$} \\
\hline$n$ & \multicolumn{2}{|c|}{543} & \multicolumn{2}{|c|}{192} & \multicolumn{2}{|c|}{76} \\
\hline Complications $\leq 7$ days & Total & By $P O A^{c}$ & Total & By POA c & Total & By POA \\
\hline Myocardial infarction (\%) & $1(0.2)$ & $1(0.2)$ & $8(4.2)$ & $2(1.0)$ & $23(30.3)$ & $16(21.1)$ \\
\hline Arrhythmia (\%) & $46(8.5)$ & $3(0.6)$ & $22(11.5)$ & $0(0.0)$ & $9(11.8)$ & $3(3.9)$ \\
\hline CPR (\%) & $0(0.0)$ & $0(0.0)$ & $2(1.0)$ & $0(0.0)$ & $2(2.6)$ & $0(0.0)$ \\
\hline Cerebrovascular accident (\%) & $9(1.7)$ & $0(0.0)$ & $2(1.0)$ & $0(0.0)$ & $3(3.9)$ & $0(0.0)$ \\
\hline Deep venous thrombosis (\%) & $4(0.7)$ & $0(0.0)$ & $0(0.0)$ & $0(0.0)$ & $0(0.0)$ & $0(0.0)$ \\
\hline Pulmonary embolism (\%) & $10(1.8)$ & $0(0.0)$ & $5(2.6)$ & $0(0.0)$ & $3(3.9)$ & $1(1.3)$ \\
\hline Sepsis (\%) & $20(3.7)$ & $0(0.0)$ & $8(4.2)$ & $1(0.5)$ & $5(6.6)$ & $1(1.3)$ \\
\hline Pneumonia (\%) & $61(11.2)$ & $6(1.1)$ & $31(16.1)$ & $0(0.0)$ & $15(19.7)$ & $2(2.6)$ \\
\hline Respiratory failure (\%) & $33(6.1)$ & $1(0.2)$ & $25(13.0)$ & $6(3.1)$ & $14(18.4)$ & $1(1.3)$ \\
\hline Acute kidney injurya (\%) & $59(15.1)$ & $1(0.0)$ & $33(21.7)$ & $0(0.0)$ & $14(22.2)$ & $4(14.8)$ \\
\hline Anaemia (\%) & $198(36.5)$ & $6(6.6)$ & $87(45.3)$ & $28(14.6)$ & $42(55.3)$ & $14(18.4)$ \\
\hline \multicolumn{7}{|l|}{ Clavien-Dindo Classification (\%) } \\
\hline Grade 1 & \multicolumn{2}{|c|}{$73(13.4)$} & \multicolumn{2}{|c|}{$16(8.3)$} & \multicolumn{2}{|c|}{$5(6.6)$} \\
\hline Grade 2 & \multicolumn{2}{|c|}{$206(37.9)$} & \multicolumn{2}{|c|}{$67(34.9)$} & \multicolumn{2}{|c|}{$25(32.9)$} \\
\hline Grade 3a & \multicolumn{2}{|c|}{$11(2.0)$} & \multicolumn{2}{|c|}{$10(5.2)$} & \multicolumn{2}{|c|}{$1(1.3)$} \\
\hline Grade 3b & \multicolumn{2}{|c|}{$26(4.8)$} & \multicolumn{2}{|c|}{$9(4.7)$} & \multicolumn{2}{|c|}{$2(2.6)$} \\
\hline Grade 4a & \multicolumn{2}{|c|}{$74(13.6)$} & \multicolumn{2}{|c|}{$47(24.5)$} & \multicolumn{2}{|c|}{$27(35.5)$} \\
\hline Grade 4b & \multicolumn{2}{|c|}{$19(3.5)$} & \multicolumn{2}{|c|}{$7(3.6)$} & \multicolumn{2}{|c|}{$8(10.5)$} \\
\hline Grade 5 & \multicolumn{2}{|c|}{$5(0.9)$} & \multicolumn{2}{|c|}{$4(2.1)$} & \multicolumn{2}{|c|}{$1(1.3)$} \\
\hline No complications & \multicolumn{2}{|c|}{$129(23.8)$} & \multicolumn{2}{|c|}{$32(16.7)$} & \multicolumn{2}{|c|}{$7(9.2)$} \\
\hline Unexpected ICU admission (\%) & \multicolumn{2}{|c|}{$21(3.9)$} & $13(6$ & (6.8) & $11(1$ & $(14.5)$ \\
\hline Unexpected MCU admission (\%) & $91(1$ & 6.8) & $5(2$ & 8.6) & $33(4$ & (43.4) \\
\hline Reoperation (\%) & $50(9$ & 9.2) & 19( & (9.9) & $8(1$ & 10.5) \\
\hline Length of stay (median [IQR]) & $7[4-$ & & $9[5$ & $-16]$ & $11[6$ & 6-16] \\
\hline Mortality within one week (\%) & $5(0$ & & $4(2$ & & & (1.3) \\
\hline
\end{tabular}

\footnotetext{
a Acute kidney injury (AKI) was calculated based on the KDIGO criteria. (1) AKI was based on 607 patients as no pre-and/or postoperative creatinine was available in 204 patients (25\%). ${ }^{b}$ Anaemia was defined as a haemoglobin $<6.0 \mathrm{mmol} \mathrm{L}-1$ (10 g dL-1), which is based on 708 patients as haemoglobin was not measured in 103 patients (13\%). c Early detection of complication (within three days) through contribution of the dedicated team of anaesthesiologist (POA: perioperative anaesthesiologist) ${ }^{d}$ Including end-stage kidney disease and end-stage vascular disease.

CPR: cardiopulmonary resuscitation. ICU; intensive care unit. IQR; interquartile range. MCU; medium care unit. NA: not applicable. Tnl: troponin I. Troponin thresholds were chosen based on the thresholds as defined in the local protocol of the University Medical Center Utrecht (Tnl 18-119 ng L ${ }^{-1}$, Tnl $120-599 \mathrm{ng} \mathrm{L}^{-1}$ and $T \mathrm{nl} \geq 600 \mathrm{ng} \mathrm{L}^{-1}$ ).
} 


\section{Postoperative consultation}

A total of 509 patients (63\%) received consultation by a dedicated anaesthesiologist (Table 3). In 35 patients (7\%), the anaesthesiologists provided advice as recorded in the electronic medical file, without an actual physical visit. In 124 patients (24\%), the cardiologist was already involved in patient care prior to or simultaneously with the dedicated anaesthesiologist, and the cardiologist was consulted by the dedicated anaesthesiologist in 155 patients (30\%). In total, 187 patients (23\%) were referred to the cardiac outpatient clinic for further follow-up after discharge.

There were more consultations by the dedicated anaesthesiologists in patients with $T n l \geq 600$ ng $L^{-1}$ compared with the other two categories. In addition, a cardiologist was more frequently consulted (80\% vs. 45\% and 13\% for patients with $\mathrm{Tnl} \geq 600 \mathrm{ng} \mathrm{L}^{-1}$ vs. Tnl 120-599 ng L $\mathrm{L}^{-1}$ and Tnl $18-120 \mathrm{ng} \mathrm{L}^{-1}$, respectively) or already involved (33\% vs. $32 \%$ and $19 \%$, respectively) (Table 3 ).

Additional diagnostics were ordered by the anaesthesiologist in 381 patients (75\%). An ECG was most frequently ordered (73\%), followed by additional troponin (34\%) and other cardiac enzymes (20\%). A change in treatment (i.e. a change in medication or red blood cell transfusion) was initiated by the anaesthesiologist in 131 patients (16\%), with 48 patients receiving blood products (6\%). There were more diagnostic tests ordered and treatments initiated by the dedicated anaesthesiologist in patients with higher troponin levels (Table 3).

In 449 (55\%) patients, no clear cause of the troponin elevation was identified (Table 3). In 22\% of patients it was considered to be caused by unstable perioperative hemodynamic, including hypotension and tachycardia, followed by ischemic heart disease (9\%) and AKI (7\%).

Table 3. Consultations, interventions and causes of troponin elevation assigned by the dedicated anaesthesiologist, dependent on the height of troponin elevation

\begin{tabular}{|c|c|c|c|}
\hline & $\begin{array}{c}\text { Tnl 18-119 } \\
\text { ng L L }^{-1}\end{array}$ & $\begin{array}{c}\text { Tnl } \\
120-599 \mathrm{ng} \mathrm{L}^{-1}\end{array}$ & $\begin{array}{c}\mathrm{TnI} \geq 600 \\
n \mathrm{~g} \mathrm{~L}^{-1}\end{array}$ \\
\hline$n$ & 543 & 192 & 76 \\
\hline \multicolumn{4}{|l|}{ Consultation (\%) } \\
\hline No visit, advice only & $28(5.2)$ & $4(2.1)$ & $3(3.9)$ \\
\hline Only 1-time visit & $186(34.3)$ & $90(46.9)$ & $39(51.3)$ \\
\hline$>1$ visit & $88(16.2)$ & $49(25.5)$ & $22(28.9)$ \\
\hline Any diagnostics ordered (\%) & $207(38.1)$ & $126(65.6)$ & $48(63.2)$ \\
\hline ECG & $201(37.0)$ & $120(62.5)$ & $47(61.8)$ \\
\hline Echocardiography & $3(0.6)$ & $19(9.9)$ & $26(34.2)$ \\
\hline CT angiography & $2(0.4)$ & $3(1.6)$ & $4(5.3)$ \\
\hline
\end{tabular}


Table 3. Consultations, interventions and causes of troponin elevation assigned by the dedicated anaesthesiologist, dependent on the height of troponin elevation

\begin{tabular}{|c|c|c|c|}
\hline & $\begin{array}{c}\text { Tnl 18-119 } \\
n^{\prime} ~ L^{-1}\end{array}$ & $\begin{array}{c}\text { Tnl } \\
120-599 \mathrm{ng} \mathrm{L}^{-1}\end{array}$ & $\begin{array}{c}\mathrm{TnI} \geq 600 \\
\mathrm{ng} \mathrm{L}^{-1}\end{array}$ \\
\hline Additional troponin & $76(14.0)$ & $61(31.8)$ & $38(50.0)$ \\
\hline Other enzymes (i.e. CK-MB) & $23(4.2)$ & $43(22.4)$ & $36(47.4)$ \\
\hline \multicolumn{4}{|l|}{ Other specialty consulted (\%) } \\
\hline Cardiology & $39(7.2)$ & $65(33.9)$ & $51(67.1)$ \\
\hline Pulmonology & $5(0.9)$ & $2(1.0)$ & $0(0.0)$ \\
\hline Other $^{a}$ & $6(1.1)$ & $2(1.0)$ & $0(0.0)$ \\
\hline Cardiology already involved (\%) & $57(10.5)$ & $46(24.0)$ & $21(27.6)$ \\
\hline Any change in treatment (\%) & $55(10.1)$ & $47(24.5)$ & $29(38.2)$ \\
\hline Change in medication & $24(4.4)$ & $26(13.5)$ & $24(31.6)$ \\
\hline Red blood cell transfusion & $6(6.6)$ & $28(14.6)$ & $14(18.4)$ \\
\hline Follow up at outpatient cardiac clinic (\%) & $73(13.4)$ & $64(33.3)$ & $50(65.8)$ \\
\hline \multicolumn{4}{|l|}{ Cause of myocardial injury (\%) } \\
\hline Ischemic heart disease & $6(1.1)$ & $12(6.2)$ & $28(36.8)$ \\
\hline Arrhythmia & $22(4.1)$ & $8(4.2)$ & $0(0.0)$ \\
\hline Congestive heart failure & $6(1.1)$ & $2(1.0)$ & $3(3.9)$ \\
\hline Pulmonary embolism & $4(0.7)$ & $1(0.5)$ & $2(2.6)$ \\
\hline Pneumonia & $7(1.3)$ & $4(2.1)$ & $0(0.0)$ \\
\hline Respiratory failure & $11(2.0)$ & $9(4.7)$ & $1(1.3)$ \\
\hline Sepsis & $9(1.7)$ & $6(3.1)$ & $2(2.6)$ \\
\hline Acute kidney injury & $31(5.7)$ & $6(3.1)$ & $0(0.0)$ \\
\hline Anaemia & $15(2.8)$ & $8(4.2)$ & $4(5.3)$ \\
\hline Fluid overload & $4(0.7)$ & $4(2.1)$ & $0(0.0)$ \\
\hline Hypertension & $6(1.1)$ & $3(1.6)$ & $0(0.0)$ \\
\hline Perioperative hemodynamics ${ }^{b}$ & $66(12.2)$ & $37(19.3)$ & $9(11.8)$ \\
\hline Other ${ }^{c}$ & $15(2.8)$ & $8(4.2)$ & $3(3.9)$ \\
\hline Cause unknown & $341(62.8)$ & $84(43.8)$ & $24(31.6)$ \\
\hline
\end{tabular}

${ }^{a}$ Including nephrologist, haematologist, cardiothoracic surgeon and geriatrician. ${ }^{b}$ Including hypotension and tachycardia. "Including pericarditis, myocardial contusion, neurological conditions (e.g. subarachnoid haemorrhage) and fever. ECG; electrocardiogram. CT; computed tomography. CK-MB; creatine-kinase isoenzyme. MCU: surgical Medium Care Unit. PACU: Post Anaesthesia Care Unit. Tnl: troponin I. Troponin thresholds were chosen based on the thresholds as defined in the local protocol of the University Medical Center Utrecht (TnI 18-119 $n g L^{-1}$, Tnl 120-599 $n g L^{-1}$ and $T n l \geq 600 n g L^{-1}$. 


\section{DISCUSSION}

This study evaluated the contribution of follow-up by dedicated anaesthesiologists on postoperative care utility and early detection of complications in patients with elevated troponin after non-cardiac surgery. The anaesthesiologists were primarily responsible for the early detection of $12 \%$ of all postoperative complications within one week after surgery, with an especially large contribution (59\%) to detection of myocardial infarctions.

\section{Literature}

The current study found that routine postoperative troponin surveillance, supported by routine postoperative consultations by a dedicated anaesthesiologist can be used to detect postoperative complications at an early stage. Increasing rates of both cardiovascular, i.e. myocardial infarction and pulmonary embolism, and non-cardiac complications, i.e. sepsis, respiratory failure, renal failure and anaemia, were found in patients with elevated troponin in a dose-dependent manner. Substantially more patients with postoperative troponin elevation were consulted after implementation of a dedicated anaesthesia team to conduct these visits ( $41 \%$ were consulted by cardiologists in the study by Van Waes et al. (9) versus 63\% in the current study).

Around $15 \%$ of patients with elevated troponin received a change in medication, which is in accordance with a previous study. (9) Six percent received red blood cell transfusion after involvement of the dedicated anaesthesiologist, aiming at a haemoglobin level $>6 \mathrm{mmol} \mathrm{L}^{-1}$ $\left(10 \mathrm{gLL}^{-1}\right)$. Interestingly, a previous study found that the cardiologist only advised red blood cell transfusion in $2 \%$ of patients with postoperative troponin elevation. (3)

\section{Clinical implications}

Prediction of the risk of postoperative complications has been shown to be difficult using only preoperative parameters.(Wijeysundera 2016) Although preoperative patient optimization and planning of perioperative care might have a larger effect on clinical outcome and costs than postoperative visits by trained anesthesiologist solely,(Dexter and Wachtel 2014) we do believe there may be an additive beneficial effect provided by these visits. Ideally, one might argue that anesthesiologists should consult all patients receiving intermediate or high-risk surgery, but resources are scarce. By identifying patients at risk not only before surgery, but again in the postoperative phase using additional data, resources can be directed towards where needed most. Troponin surveillance may be an efficient manner to conduct this postoperative selection. (Devereaux et al. 2017; van Waes et al. 2016; Weber et al. 2013) Therefore, we focused on patients with elevated troponins to select those at risk of postoperative complications and to first determine the effect of this intervention, before unrolling it to a broader patient population. 
The present study found that follow-up by dedicated anesthesiologists enabled early detection of $12 \%$ of all complications and $59 \%$ of all postoperative myocardial infarctions within one week after surgery. The experience of anesthesiologists with procedure-related complications in addition to their knowledge of the cardiopulmonary and central nervous system can provide a valuable contribution to postoperative care by surgeons and care by cardiologists in case of postoperative troponin elevation. In our institution, the resources needed for a dedicated team of anesthesiologists conducting postoperative visits in this group of patients are low. One dedicated anesthesiologist on call spends on average one hour per day on screening and followup of patients with postoperative troponin elevation. In case of any emergent complications caught by this anesthesiologist, additional time is needed for extra diagnostics, consultation of other medical specialties and follow-up. The remaining time is spent on clinical tasks at the outpatient clinic not evaluated in this study, such as preoperative assessment of patients and multidisciplinary meetings. In our center, no extra personnel needed to be employed to implement this program. In addition, a cardiologist was consulted in only $30 \%$ of patients instead of $41 \%$ as reported previously.(van Waes et al. 2016) Although additional diagnostic procedures were performed, these were mostly low-cost such as an ECG, while the number of patients in whom a complication was diagnosed and medical treatment was changed, was substantial. Importantly, in a majority of the patients (55\%) a clear cause of troponin elevation could not be identified.

Therefore, treatment options currently are often limited. Prospective studies are necessary to further determine the potential effect of postoperative visits by dedicated anesthesiologist on patient outcome.

\section{Strengths and Limitations}

To the best of our knowledge, this is the first study reporting on the effect of postoperative anaesthesia visits on resource utility and detection of postoperative complications in patients with postoperative troponin elevation. It was conducted in the non-cardiac surgery population, making these results generalizable to a large group of patients.

This study has some important limitations. First, because of the retrospective design of the study and limited number of patients, we were unable to estimate whether the implementation of routine postoperative anaesthesia visits resulted in better patient outcomes. Second, two different troponin assays, i.e. normal Tnl and the high sensitive Tnl, were used during the inclusion period. As the second assay is more sensitive, this might have resulted in a higher incidence of troponin elevation. However, there were no differences in preoperative characteristics, but we did find more myocardial infarctions in the period the normal Tnl assay was used together with more cardiology consultations, extra ordered diagnostics and change in medication. Third, many patients were referred to the cardiac outpatient clinic for further follow-up, but often this was conducted in other institutions, since the UMC Utrecht is a tertiary referral hospital. This 
hampered our ability to track down diagnostics and interventions instituted during these followup visits. Fourth, there were some missing values for creatinine and haemoglobin. Incidence rates of postoperative anaemia and AKI are probably overestimated as their markers were mostly missing in healthier patients. Fifth, because myocardial infarction was defined as a clinical diagnosis made by a cardiologist, the incidence of myocardial infarction may have been higher when it retrospectively would have been based on the $4^{\text {th }}$ universal definition (8), as previously shown. $(3,6,11)$ Finally, only documented consultations by the anaesthesiologist were recorded as consultation, which potentially explains why $37 \%$ of the patients did not receive a consultation. As part of the local protocol, anaesthesiologists determined whether consultation was necessary based on clinical course reported in the electronic medical files and consultation of treating physicians by phone. We presume that in patients with elevated but low troponin levels (especially with Tnl 19-120 ng/l), an ECG was remotely assessed by the anaesthesiologist and discussed with the treating physician. In case of no abnormalities nothing was documented in the electronical medical files, although in fact remote consultation did occur.

\section{CONCLUSION}

Implementation of routine postoperative consultations by dedicated anaesthesiologists resulted in early detection and treatment of complications in patients with postoperative elevated troponin levels. 


\section{REFERENCES}

1. Devereaux PJ, Biccard BM, Sigamani A, Xavier D, Chan MTV, Srinathan SK, et al. Association of Postoperative High-Sensitivity Troponin Levels With Myocardial Injury and 30-Day Mortality Among Patients Undergoing Noncardiac Surgery. Jama. 2017;317(16):1642-51.

2. Levy M, Heels-Ansdell D, Hiralal R, Bhandari M, Guyatt G, Yusuf S, et al. Prognostic value of troponin and creatine kinase muscle and brain isoenzyme measurement after noncardiac surgery: a systematic review and meta-analysis. Anesthesiology. 2011;114(4):796-806.

3. van Waes JAR, Grobben RB, Nathoe HM, Kemperman H, de Borst GJ, Peelen LM, et al. OneYear Mortality, Causes of Death, and Cardiac Interventions in Patients with Postoperative Myocardial Injury. Anesthesia and Analgesia. 2016;123(1):29-37.

4. van Waes JAR, Nathoe HM, de Graaff JC, Kemperman H, de Borst GJ, Peelen LM, et al. Myocardial Injury After Noncardiac Surgery and its Association With Short-Term Mortality. Circulation. 2013;127(23):2264-71.

5. Devereaux PJ, Chan MTV, Alonso-Coello P, Walsh M, Berwanger O, Villar JC, et al. Association between postoperative troponin levels and 30-day mortality among patients undergoing noncardiac surgery. JAMA - Journal of the American Medical Association. 2012;307(21):2295304.

6. Devereaux PJ, Xavier D, Pogue J, Guyatt G, Sigamani A, Garutti I, et al. Characteristics and short-term prognosis of perioperative myocardial infarction in patients undergoing noncardiac surgery: a cohort study. Ann Intern Med. 2011;154(8):523-8.

7. Duceppe E, Parlow J, MacDonald P, Lyons K, McMullen M, Srinathan S, et al. Canadian Cardiovascular Society Guidelines on Perioperative Cardiac Risk Assessment and Management for Patients Who Undergo Noncardiac Surgery. Canadian Journal of Cardiology. 2017;33(1):17-32.

8. Thygesen K, Alpert JS, Jaffe AS, Chaitman BR, Bax JJ, Morrow DA, et al. Fourth Universal Definition of Myocardial Infarction (2018). Circulation. 2018;138(20):e618-e51.

9. van Waes JAR, van Klei WA, Wijeysundera DN, van Wolfswinkel L, Lindsay TF, Beattie WS. Association between Intraoperative Hypotension and Myocardial Injury after Vascular Surgery. Anesthesiology. 2016;124(1):35-44.

10. Akkermans A, Peelen LM, van Waes JA, Rinkel GJ, van Klei WA. Cardiac events within one year after a subarachnoid haemorrhage: The predictive value of troponin elevation after aneurysm occlusion. Eur J Prev Cardiol. 2019;26(4):420-8.

11. Beattie WS, Wijeysundera DN, Chan MTV, Peyton PJ, Leslie K, Paech MJ, et al. Implication of Major Adverse Postoperative Events and Myocardial Injury on Disability and Survival: A Planned Subanalysis of the ENIGMA-II Trial. Anesth Analg. 2018;127(5):1118-26.

12. Noordzij PG, van Geffen O, Dijkstra IM, Boerma D, Meinders AJ, Rettig TCD, et al. High-sensitive cardiac troponin T measurements in prediction of non-cardiac complications after major abdominal surgery. British Journal of Anaesthesia. 2015;114(6):909-18.

13. von Elm E, Altman DG, Egger M, Pocock SJ, Gotzsche PC, Vandenbroucke JP. The Strengthening the Reporting of Observational Studies in Epidemiology (STROBE) statement: guidelines for reporting observational studies. J Clin Epidemiol. 2008;61(4):344-9. 
14. Lee TH, Marcantonio ER, Mangione CM, Thomas EJ, Polanczyk CA, Cook EF, et al. Derivation and prospective validation of a simple index for prediction of cardiac risk of major noncardiac surgery. Circulation. 1999;100(10):1043-9.

15. Anesthesiologists ASo. ASA Physical Status Classification System. 2014.

16. Kristensen SD, Knuuti J, Saraste A, Anker S, Botker HE, De Hert S, et al. 2014 ESC/ESA Guidelines on non-cardiac surgery: cardiovascular assessment and management: The Joint Task Force on non-cardiac surgery: cardiovascular assessment and management of the European Society of Cardiology (ESC) and the European Society of Anaesthesiology (ESA). Eur J Anaesthesiol. 2014;31(10):517-73.

17. Dindo D, Demartines N, Clavien PA. Classification of surgical complications: a new proposal with evaluation in a cohort of 6336 patients and results of a survey. Ann Surg. 2004;240(2):20513.

18. Jammer I, Wickboldt N, Sander M, Smith A, Schultz MJ, Pelosi P, et al. Standards for definitions and use of outcome measures for clinical effectiveness research in perioperative medicine: European Perioperative Clinical Outcome (EPCO) definitions: a statement from the ESAESICM joint taskforce on perioperative outcome measures. Eur J Anaesthesiol. 2015;32(2):88105.

19. CBO. Richtlijn Bloedtransfusie (Guideline blood transfusion). 2011;182-3.

20. Wijeysundera DN. Predicting outcomes: Is there utility in risk scores? Canadian Journal of Anesthesia-Journal Canadien D Anesthesie. 2016;63(2):148-58.

21. Dexter F, Wachtel RE. Strategies for net cost reductions with the expanded role and expertise of anesthesiologists in the perioperative surgical home. Anesth Analg. 2014;118(5):1062-71.

22. Weber M, Luchner A, Manfred S, Mueller C, Liebetrau C, Schlitt A, et al. Incremental value of high-sensitive troponin $\mathrm{T}$ in addition to the revised cardiac index for peri-operative risk stratification in non-cardiac surgery. European Heart Journal. 2013;34(11):853-62. 


\section{CHAPTER}

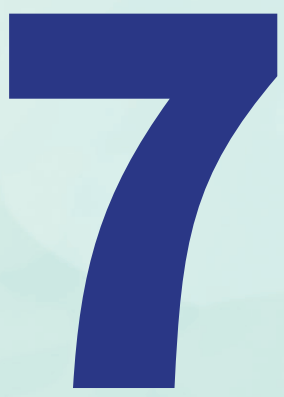

Association between postoperative myocardial injury phenotypes and disability-free survival in patients undergoing non-cardiac surgery

Lisette M. Vernooij Judith A.R. van Waes

Wilton A. van Klei Linda M. Peelen W. Scott Beattie 


\section{ABSTRACT}

Background: Postoperative myocardial injury (PMI) is associated with postoperative morbidity and mortality. The most common phenotype of PMI is myocardial infarction (MI), however PMI has also been related to other cardiac and non-cardiac pathologies. The patient centred impact of these PMI phenotypes is largely unknown. Therefore, we examined the independent effects of PMI phenotypes on disability-free survival following non-cardiac surgery.

Methods: This multicentre prospective cohort study included 653 patients ( $\geq 50$ years) undergoing elective major non-cardiac surgery. Patients were stratified based on PMI and the occurrence of postoperative adverse events. This resulted in five groups, i.e. no adverse events (1), non-MI events with PMI (2), MI (3), isolated PMI (4) PMI, and (5) non-MI events with PMI. The primary outcome was disability-free survival 6 months after surgery. Multivariable Poisson regression was used to investigate the association between PMI phenotypes and disability-free survival.

Results: PMI occurred in 125 (19\%) patients and 485 (74\%) had disability-free survival at 6 months. None of the PMI phenotypes were associated with a reduction in disability-free survival, i.e. myocardial infarction (OR: 1.5, 95\% Cl 0.7-2.9, $\mathrm{p}=0.26$ ), non-MI complications with PMI (OR: 1.6, 95\% Cl 0.8-2.8, p=0.15) non-MI complications without PMI (OR: 1.5, 95\% Cl: 1.0-2.3, p=0.06) and isolated PMI (OR: 1.1, 95\% Cl 0.6-1.8, p=0.78). Besides cardiac ischemia, patients with PMI were also often diagnosed with pneumonia, respiratory failure and acute kidney injury.

Conclusion: In our study, postoperative complications were found to increase death and disability. No association was found for the PMI phenotypes probably due the low number of patients in these groups. Early recognition and management of cardiac and non-cardiac complications in patients at high risk might benefit disability-free survival on the long-term. 


\section{INTRODUCTION}

Annually, several hundred million patients undergo non-cardiac surgery worldwide. $(1,2)$ Despite the potential benefits aimed for, approximately 1 out of 6 patients undergoing surgery will develop a complication. (3) Complications most commonly have an infectious (33\%) or cardiac origin (19\%). (3) Perioperative cardiovascular events are a leading cause of morbidity and mortality after non-cardiac surgery (4-10) and are associated with prolonged hospitalization and increased medical costs. (11) However, cardiac complications are hard to diagnose, as typical symptoms are often not present in postoperative patients (e.g., chest pain may be masked by the analgesic and sedative effects of anaesthetics and distracting pain from the surgical wound). (7) Consequently, postoperative troponin surveillance is recommended to identify patients at risk for such complications. $(12,13)$

Postoperative myocardial injury (PMI), defined as elevated troponin, occurs in 11-19\% of patients and is independently associated with morbidity and mortality, in a concentration-dependent manner. (4-10) PMI has a heterogeneous appearance with a broad spectrum of different phenotypes. (12) The best known phenotype of PMI is myocardial infarction (MI). Other PMI phenotypes are among others, cardiac pathologies related to heart failure and arrhythmia and non-cardiac disease entities including respiratory failure, pulmonary embolism, renal failure and sepsis. $(4,10,12)$

Nowadays, the patient-centred outcomes including disability status after surgery seem to be more important than clinical outcomes such as myocardial infarction, especially as the consequences of different clinical outcomes on disability on the long term differ considerably. (10, 14) Disability is defined as difficulties experienced by a patient in different functional domains, including cognition, mobility, self-care, getting along, life activities and participation during the previous 30 days. (15)

The long term consequences of experiencing any type of PMI with respect to disability are not yet understood. Therefore, our primary objective was to investigate and compare the independent effects of the different PMI phenotypes on 6-month disability-free survival in patients undergoing non-cardiac surgery. We aimed to diagnose the different PMI phenotypes using clinical evaluation and biomarkers. Our primary hypothesis was that any postoperative troponin elevation regardless of its association with myocardial infarction or non-cardiac adversity is independently associated with an increased risk of death and disability. 


\section{METHODS}

\section{Study design and population}

This multicentre prospective cohort study included patients undergoing non-cardiac surgery at the University Health Network Toronto (UHNT), Toronto Canada from June 26, 2014 to June 12, 2015 and at the University Medical Center Utrecht (UMCU), Utrecht, the Netherlands, from January 29, 2018 to June 18, 2019. Eligible patients ( $\geq 50$ years) were those scheduled for elective intermediate to high risk non-cardiac surgery under general or spinal anaesthesia with an expected postoperative hospital stay of at least 24 hours. In the UMCU, only patients $\geq 60$ years were included as those patients are part of a standard postoperative troponin monitoring protocol.

All participants provided written informed consent and the local ethics committees of both hospitals approved the study protocol prior to patient recruitment (Utrecht Medical Research Ethics Committee, protocol number 17-673/M). Before initiation, the study was registered on ClinicalTrials.gov (NCT02146560 and NCT03408522)

\section{Procedures}

Before and after surgery, patients underwent daily blood sampling until the third postoperative day or hospital discharge (whichever came first). At postoperative day 1, an electrocardiogram (ECG) was made and blindly assessed by a cardiologist for signs of ischemia. Biomarkers measured were high sensitive troponin I (hsTnl) and creatinine. Creatinine was analysed using the Creatinine assay and hsTnl was analysed using the ARCHITECT STAT High Sensitive Troponin-I assay (Abbott Diagnostics, Lisnamuck, Longford, Ireland). In the analysis, the highest value of all postoperative measurements for each patient was used. Treating physicians were blinded to the biomarker results except if they requested measurement of (one or more) biomarkers as part of clinical care. In the UMCU, hSTnl is routinely measured for all participants as part of a standard protocol and accordingly is always available in clinical practice. Postoperative myocardial injury (PMI) was defined as elevated hsTnl exceeding the $99^{\text {th }}$ percentile of the used assay with a $10 \%$ coefficient of variation. Additional diagnostic tests or initiation of therapy was left at the cardiologist's discretion which was done in deliberation with the treating physician.

\section{Data collection}

All data were collected from electronic medical and administrative records. Data were obtained for demographics, comorbidities including active malignancy and the Revised Cardiac Risk factors, (16) chronic medication use (i.e. beta blockers, calcium channel blockers, angiotensin converting enzyme inhibitors, anti-platelets and statins), surgical characteristics (i.e. specialty, open or laparoscopic procedure, type of anaesthesia), and in-hospital complications. History 
of ischemic heart disease was defined as previous myocardial infarction and/or coronary revascularization, systolic heart failure was defined as a left ventricular ejection fraction $<40 \%$. Complications collected were both cardiac and non-cardiac adverse events. These included myocardial infarction defined by the fourth universal definition, (12) heart failure requiring consultation and treatment by a cardiologist, arrhythmia diagnosed on 12-leads ECG or cardiac monitor, cerebrovascular accident (defined as radiologically confirmed ischemic or haemorrhagic stroke or transient ischemic attack), radiologically confirmed deep venous thrombosis and pulmonary embolism, sepsis as clinically diagnosed by the treating physician and requiring ICU admission, respiratory failure requiring ICU admission, pneumonia requiring antibiotics and acute kidney injury (AKI) defined as an increase in creatinine of $26.4 \mathrm{mmol} / \mathrm{L}$ or $25 \%$ from the preoperative creatinine value. (17) In addition, we also collected data on intraoperative blood loss, length of hospital stay, reoperations and in-hospital mortality.

\section{Outcomes}

Disability was assessed using the 12-item World Health Organization Disability Assessment Score 2.0 (WHODAS 2.0).(15) The WHODAS 2.0 is an easy to use and clinically valid tool for measuring postoperative disability. It was surveyed twice, i.e. before and at 6 months after surgery, and scored as previously described and calculated to a percentage. (15) A WHODAS 2.0 score of 0\% was defined as having no disability. Patients who died were defined as fully disabled and were therefore appointed a score of 100\%. (15) Data on 6-months mortality were retrieved through the electronic medical records and the municipal personal records database. Moderate disability was defined as a score $\geq 25 \%$ and $<50 \%$ and severe disability as a score $\geq 50 \%$. (15)

The primary outcome of this study was (new) disability-free survival at 6 months defined as:

being alive

postoperative WHODAS 2.0 score $<25 \%$ that has not increased by $\geq 8 \%$ from baseline; postoperative WHODAS score $\geq 25 \%$ that has improved from baseline (14)

This means, for example, that a patient with a preoperative score of $80 \%$ who improved to a score of $40 \%$ at 6 months was classified as having disability-free survival, whereas a patient with a preoperative score of $11 \%$ and a score of $20 \%$ at 6 months was classified as being disabled. As a secondary outcome, we used the change in WHODAS score at 6 months relative to the preoperative measurement. A change of $\geq 8 \%$ was interpreted as clinically relevant. (14)

\section{Statistical analysis}

Patients were divided into groups based on predefined PMI phenotypes, which entailed the combination of an elevation in hsTnl and the occurrence of postoperative adverse events. 
Postoperative adverse events included in-hospital death, myocardial infarction, heart failure, arrhythmia, deep venous thrombosis, cerebrovascular accident, pulmonary embolism, acute kidney injury, respiratory failure, pneumonia, sepsis and reoperation. This resulted in the categorization of patients into five groups, i.e. no adverse events (1), non-MI events without PMI (2), MI (3), isolated PMI (4) PMI, and (5) non-MI events with PMI. Baseline characteristics were compared among the different groups. Categorical variables were compared using the Chi-square test and continuous variables were compared using the one-way ANOVA and Kruskal-Wallis test, as appropriate.

Univariable regression analysis was conducted to investigate the crude association between PMI phenotypes and disability-free survival. Thereafter, multivariable Poisson regression analysis was performed for the same association with adjustment of predefined confounders. These included preoperative demographics such as age, sex, preoperative WHODAS 2.0 score, the separate RCRI factors, (16) cancer diagnosis and surgical specialty. Surgical specialties included are ear, nose, throat (ENT) and dental, general, gynaecology and urology, neurosurgery, orthopaedics, thoracic and vascular surgery. Patients without complications served as the reference group. As the interpretation of the estimates for disability-free survival is more difficult because it is a positive outcome, the associations are reported for no disability-free survival. The results are presented as risk ratios (RR's) with 95\% confidence intervals.

For the secondary outcome, we investigated the association between the PMI phenotypes and change in WHODAS 2.0 at 6 months relative to before surgery using general linear regression analyses. Univariable and multivariable regression analyses were performed as described in the previous analysis. The results are expressed as linear regression coefficients ( $\beta$ ) with 95\% confidence intervals $(95 \% \mathrm{Cl}$ ). Furthermore, an alluvial plot was made to visually present the change in disability status from preoperative to 6 months after surgery dependent on the different aforementioned PMI phenotypes. In short, alluvial plots are a type of flow diagram originally developed to represent changes in network structure over time which is especially suitable for categorical data. A p-value of 0.05 was considered as statistically significant. The statistical analyses were performed using R statistics (Version 3.5.1 @ 2018-07-02, R, Inc., for Windows).

\section{RESULTS}

\section{Patient flow and baseline characteristics}

In total, 764 patients undergoing intermediate to high risk non-cardiac surgery provided written informed consent, of which 111 (14.5\%) were excluded. Reasons for exclusions were lost-tofollow up ( $n=51,46 \%$ ), data was not available at time of analysis ( $n=44,40 \%)$, refuse to fill out the 6 -months WHODAS 2.0 ( $n=11,10 \%)$ or discharge at the same day as hospitalization $(n=5,5 \%)$. 
Thus, 653 patients were included in the analysis, of which 422 patients were treated at the UMCU and 231 patients at the UHNT.

Troponin was elevated in 125 (19\%) patients and 101 (16\%) and 29 (4\%) were moderately and severely disabled before surgery, respectively (Figure 1). During hospitalization, 140 (21\%) patients suffered from a complication of which 24 (17\%) developed a myocardial infarction. In these patients with infarction, troponin was found to be elevated before surgery in 1 patient (4\%), which was not different compared to all included patients ( $n=26,4 \%)$. Non-Ml events without PMI occurred in 83 (13\%) patients. Isolated PMI, i.e. troponin elevation in absence of myocardial infarction and other events, occurred in 68 patients (10\%) and 33 patients (5\%) had non-Ml events with PMI. Patient characteristics are described in Table 1. Included patients were more often males (59\%), underwent more frequently high risk surgery (58\%) and were $48 \%$ of patients were diagnosed with cancer. Patients with PMI (i.e. isolated PMI, MI and non-MI events with PMI) were older and had a higher RCRI score. We observed no differences among the PMI phenotypes in the specialty for which patients underwent surgery. No difference was found in the number of patients with preoperative moderate disability $(p=0.66)$, however patients with PMI were more often preoperatively disabled $(p=0.008)$. 


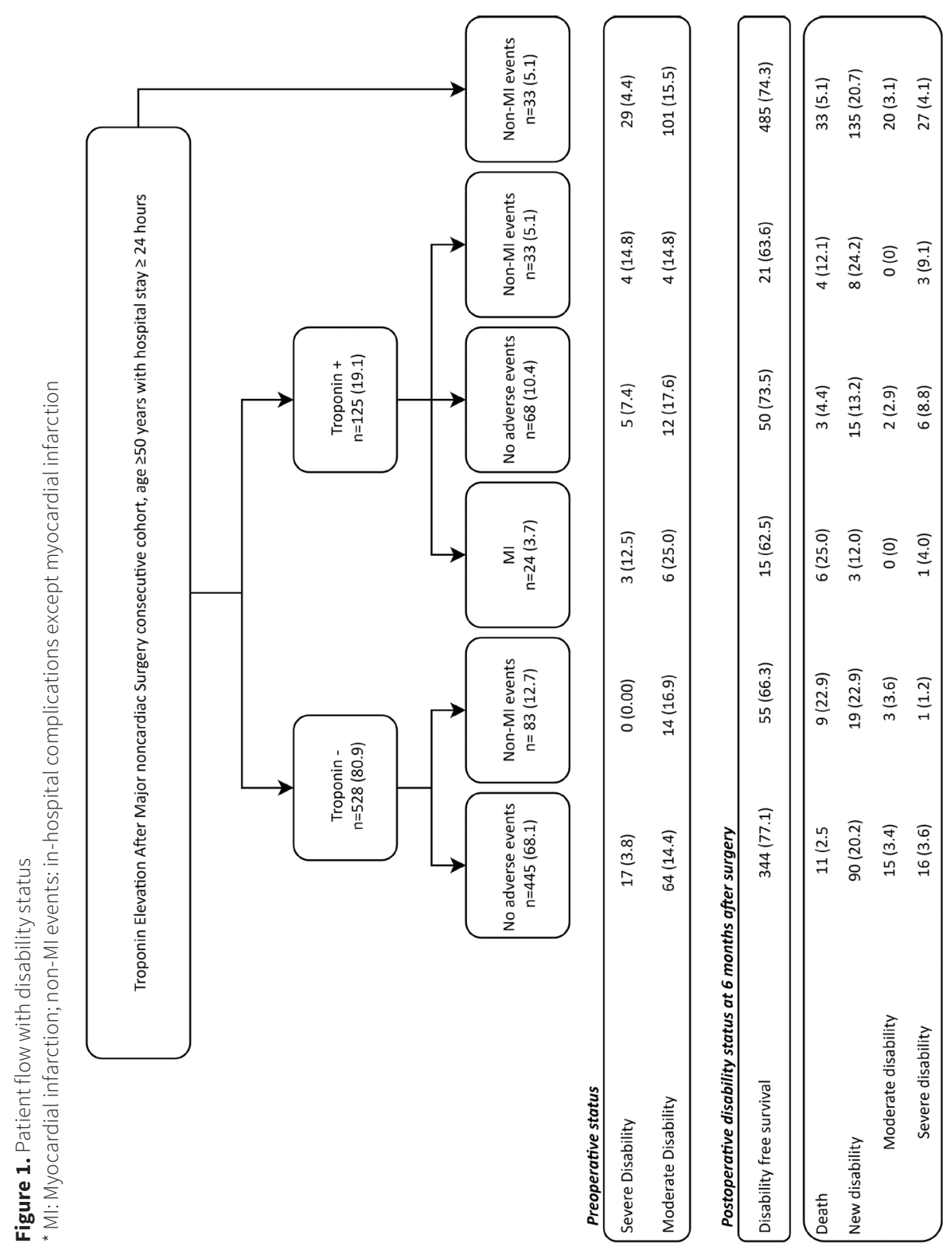




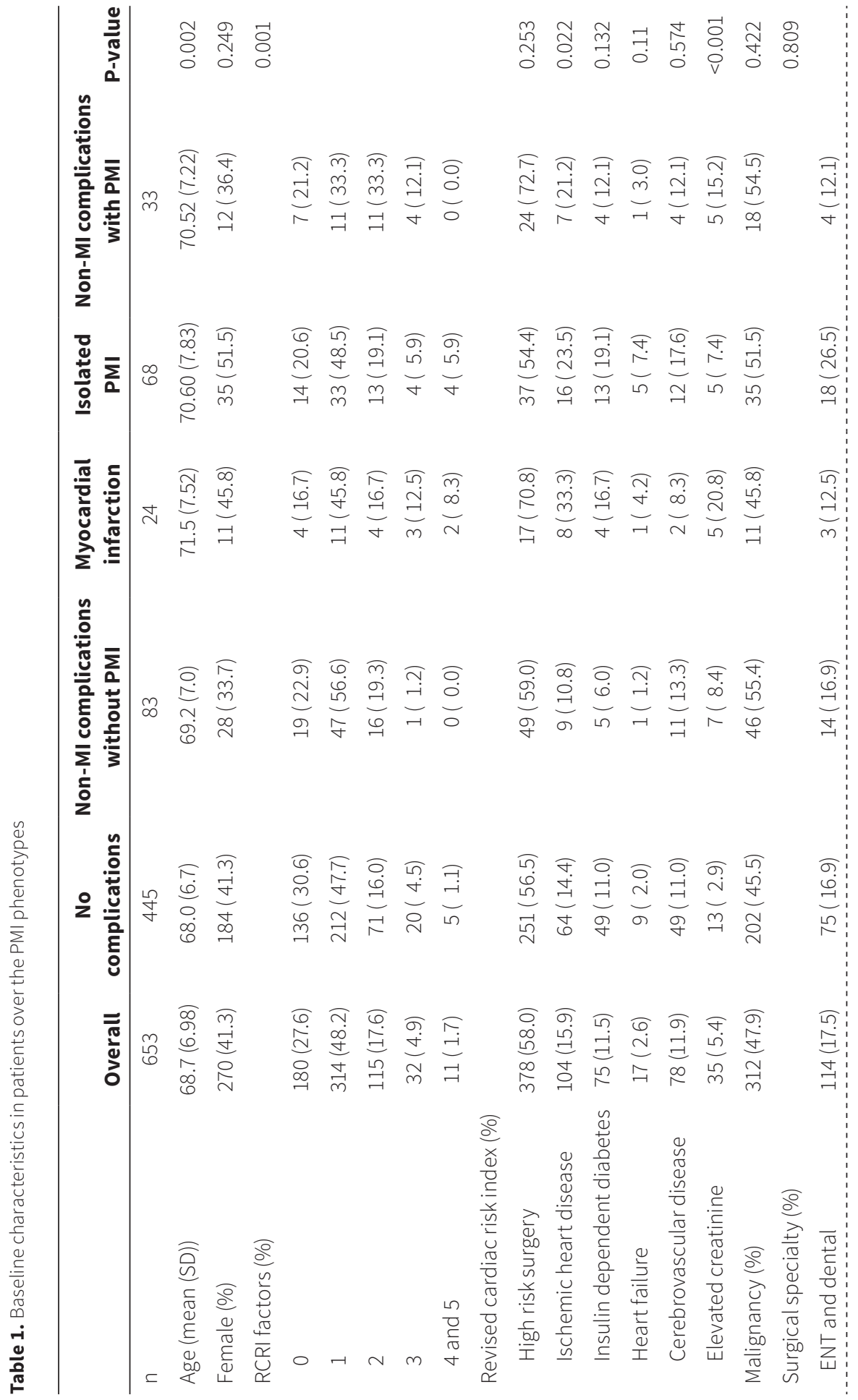




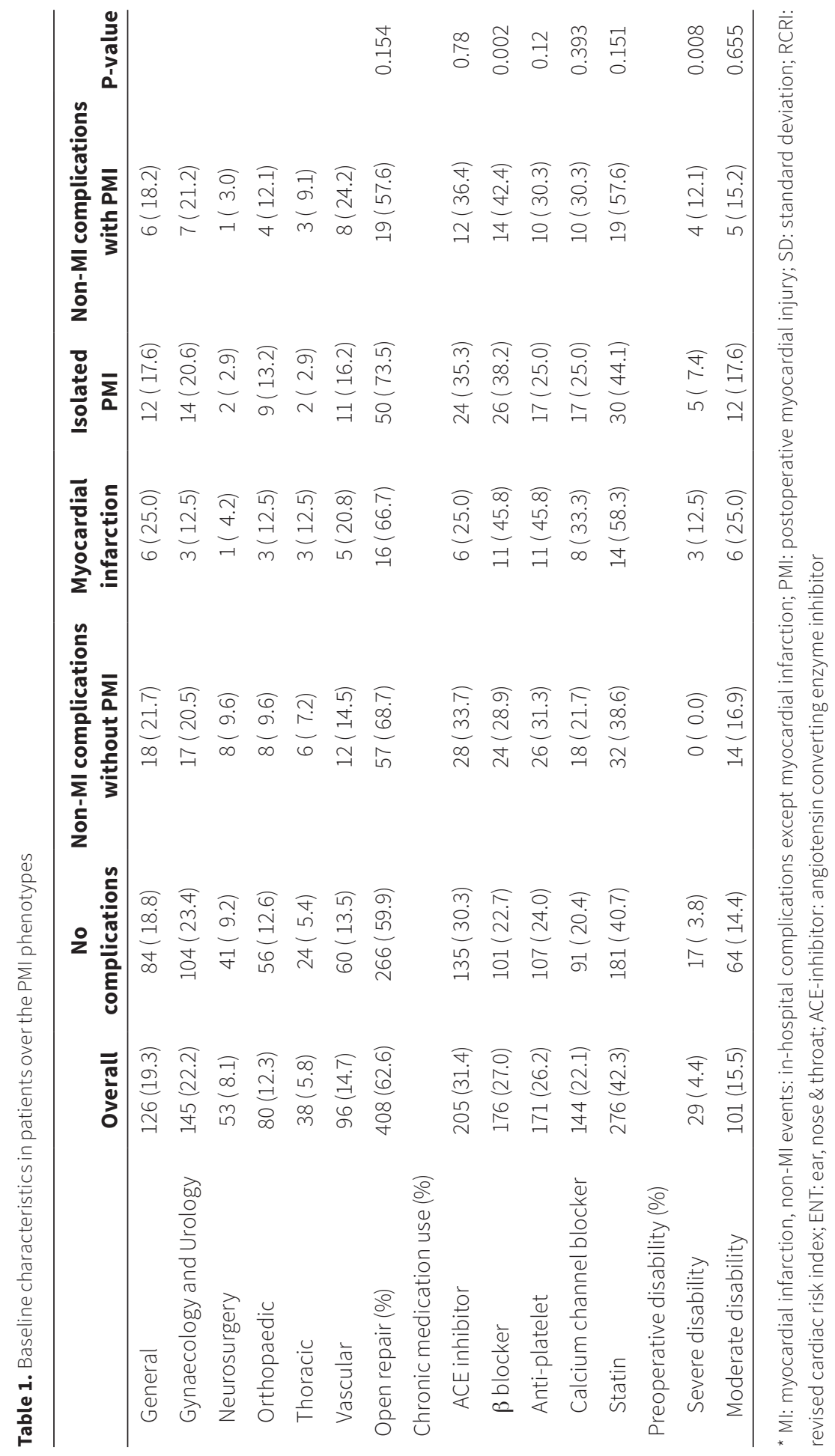




\section{In-hospital complications}

In total, 234 complications occurred in 140 patients, of which pneumonia ( $n=44,19 \%)$, reoperation $(n=37,16 \%)$ and acute kidney injury ( $n=35,15 \%)$ were diagnosed most frequently (Table 2$)$. In patients with PMI, 24 (19\%) suffered a myocardial infarction. However besides cardiac ischemia, patients with PMI also suffered more often other cardiac and non-cardiac complications compared to patients without PMI including pneumonia ( $14 \%$ and 5\%, respectively), respiratory failure (12\% and $<1 \%$ ) and arrhythmia (9\% and 2\%). Estimated blood loss during surgery was larger in patients with PMI compared to patients without PMI.

\section{Disability-free survival}

Four hundred eighty-five patients (74\%) had disability-free survival at 6 months. Figure 2 shows the trajectories of patients based on disability status from before to 6 months after surgery dependent on their PMI phenotypes during hospitalization (frequencies per stratum is available in Supplemental Table 1). Patients who died during the follow-up period were classified as severely disabled. Fortunately, the largest part of patients without preoperative disability, but also patients with preoperative moderate and severe disability had disability-free survival. Patients without in-hospital complications (i.e. no adverse events and isolated PMI) had increased disabilityfree survival compared to patients with adverse events, but this was not statistically significant $(p=0.071)$. After adjustment for confounders on the association between the PMI phenotypes and reduced disability-free survival, no statistically significant association was found for any of the PMI phenotypes. Using patients without complications as a reference, effect estimates were comparable for patients with MI (RR: 1.5 (95\% Cl 0.7-2.9, p=0.26), patients with non-MI events with (RR: 1.6 (95\% Cl 0.2-2.8, p=0.15) and without PMI (RR: 1.5 (95\% Cl 1.0-2.3, p=0.06). We found no association between isolated PMI and reduced disability-free survival (RR: 1.1 (95\% Cl 0.6-1.8, $p=0.78$ ). More information on the association with confounders can be found in Supplemental Table 2.

\section{Change in WHODAS 2.0 score}

Patients diagnosed with myocardial infarction had a 15 point ( $95 \% \mathrm{Cl}: 5.3-24.2, \mathrm{p}=0.002$ ) higher change in WHODAS 2.0 at 6 months compared to patients without complications. Significant associations were also found for patients with non-MI complications with ( $\beta$ : 9.2, Cl 95\%: 1.2-17.3, $\mathrm{p}=0.025)$ and without PMI ( $\beta$ : 7.1, CI 95\%: 1.8-12.4, p=0.009), although the latter was not interpreted as clinically relevant (i.e. change $>8 \%$ ). Again, isolated PMI was not associated with disability ( $\beta$ : 1.2, Cl 95\%: -4.7-7.0, $p=0.69$ ). 


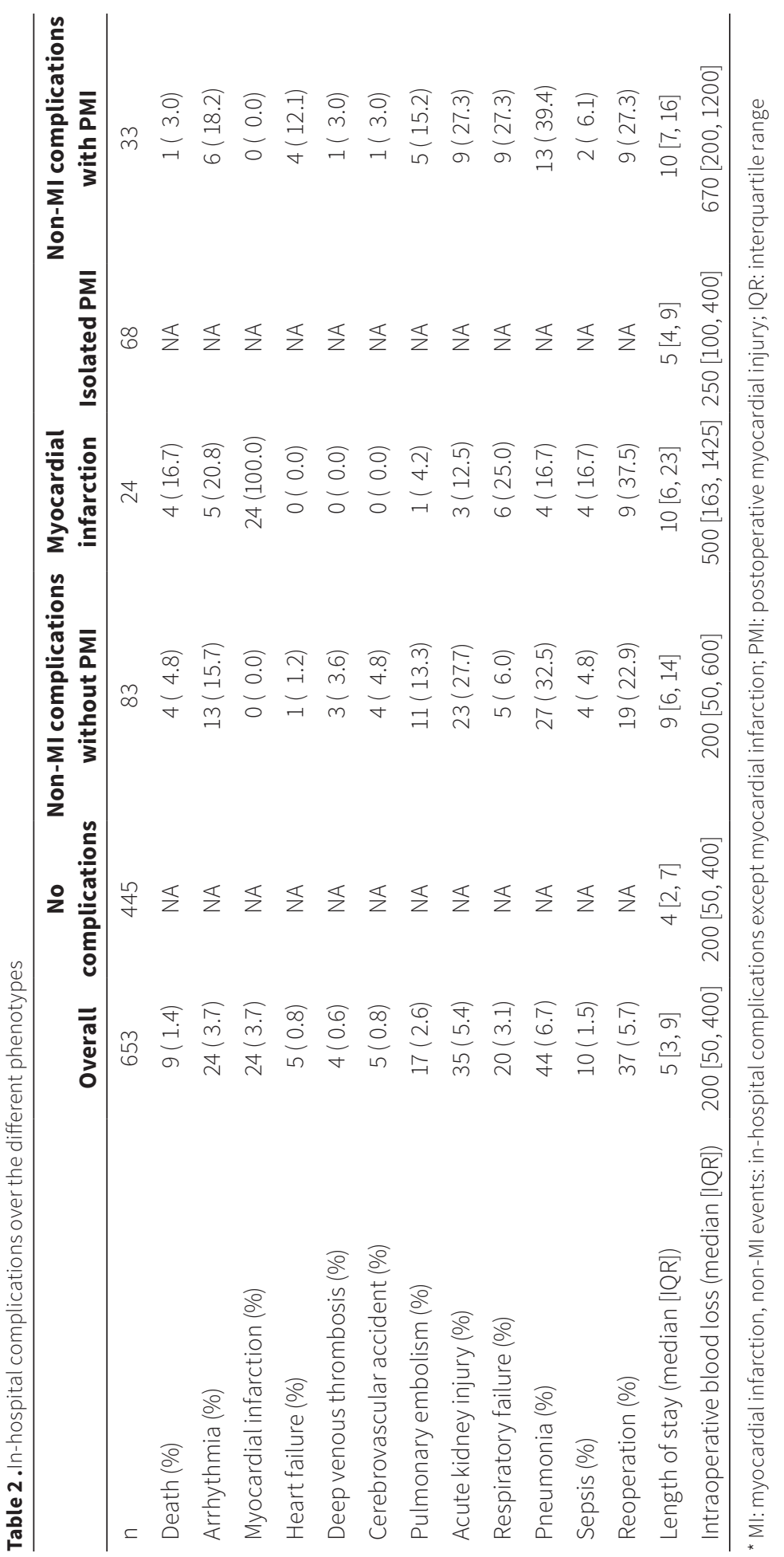



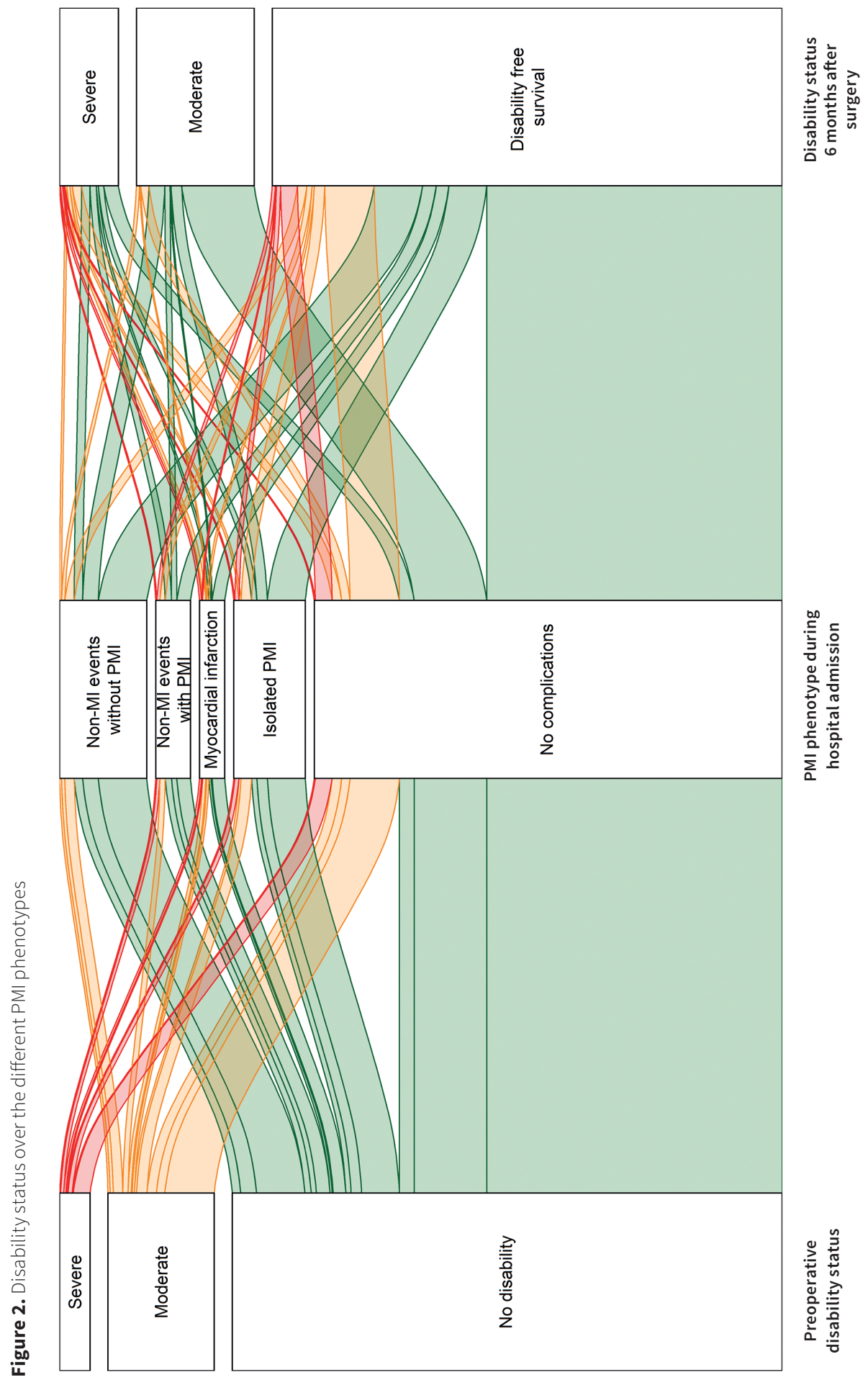

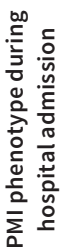




\section{DISCUSSION}

This multicentre prospective cohort study investigated the relationship between different PMI phenotypes and disability-free survival after 6 -months in patients undergoing intermediate to high risk non-cardiac surgery. Disability-free survival at 6 months was observed in 485 patients (74\%). No statistically significant differences were found among the different PMI phenotypes for its association with reduced disability-free survival. Comparable effect estimates were found in patients with complications, i.e. patients diagnosed with MI and patients with non-MI events with and without PMI. However, a clinically relevant change in WHODAS after surgery was found for patients with myocardial infarction (15\%) and patients with non-MI events with PMI (9\%). We observed no association between isolated PMI and disability, i.e. neither for disability-free survival nor for change in WHODAS 2.0 score. Patients with PMI were, besides cardiac ischemia, also often diagnosed with other cardiac and non-cardiac complications such as pneumonia, respiratory failure and acute kidney injury.

PMI has been associated with a broad spectrum of different phenotypes of which myocardial infarction is the best known. $(4,12,18)$ As most of these complications are commonly not recognized because typical symptoms are lacking, several guidelines have recommended routine postoperative cardiac biomarker surveillance to early detect postoperative complications. (12, 19) In our population, 125 (20\%) patients had an elevated troponin and without routine troponin surveillance, many complications would not have been recognized and consequently not have been treated. Patients with MI also often suffered an arrhythmia (21\%), respiratory failure (25\%), pneumonia (17\%) and sepsis (17\%) and were more frequently re-operated compared to the other phenotypes (38\% and 23\%, 27\% and 0\% for non-MI events without and with PMI and no adverse events, respectively). Besides the belief that PMI due to ischemia is only adversely associated, (7) other events including arrhythmia, respiratory failure, acute kidney injury and sepsis were also often diagnosed in patients with PMI. Therefore, we should look at PMI as a marker of adversity in patients at high risk for complications (i.e. both due to cardiac ischemia, but also other cardiac and non-cardiac events) and disability or death at the long term.

Currently, there is a shift going on from traditional outcome measures, i.e. mostly in-hospital complications, to patient reported outcome measures including return to full health, optimal functional capacity and disability free survival on the long term. (20-22) Patient reported outcomes tend to be more important as the impact of complications on long term recovery, such as myocardial infarction or stroke, may vary considerably. (14) Disability free survival has been of particular interest in multiple studies, $(23,24)$ however comparison of different types of patients in a heterogeneous surgical population is challenging. For example, a patient undergoing a hip replacement, who is disabled before surgery as he/she cannot walk, experiences disability free survival on the long term as surgery resolves the disability. Such a patient is compared to someone 
undergoing surgery for cancer treatment, who has no disability before surgery, but becomes severely disabled postoperatively due to disease progression. Beattie and colleagues (10) found that each of the PMI subtypes were associated with reduced disability free survival one year after surgery. However, the current investigation cannot reproduce this finding as no differences among the PMI phenotypes were observed. This may be due to the different tools that were used to assess disability-free survival, a different follow-up period (i.e. 6 months versus one year) and the considerably lower sample size of the current study. However, a clinical relevant difference was found for in change in WHODAS 2.0 for patients who suffered a myocardial infarction and patients with non-MI event with PMI. Larger number of patients in each of the PMI groups is needed to be able to compare its effect on disability-free survival. There are no other investigations looking at differences in disability free survival and complications after surgery based on PMI.

To the best of our knowledge, this is the first study reporting on the effect of different PMI phenotypes on disability free survival 6 months after surgery. This study is a prospective, multicentre and multicountry cohort study in the non-cardiac surgery population, making these results generalizable to a large group of patients.

Several limitations must be addressed. Due to the relatively small subgroups based on PMI phenotype, the associations found are most likely underpowered. The study was originally powered on 1000 patients and recruitment and follow-up is still ongoing at the UHNT, Canada, and the Erasmus Medical Center in Rotterdam, the Netherlands. However, the incidences of both MI and PMI in the current analyses follow similar incidences as reported in recent literature. $(4-8,10)$ Secondly, some in-hospital complications, such as pulmonary embolism or myocardial infarction occurring after the third postoperative day, might have been missed as these were not recognized in clinical practice. $(7,25,26)$ This might have resulted in an underestimation of the true incidence of these complications. Third, the postoperative WHODAS 2.0 score was missing in 111 (15\%) patients, of whom 50\% refused to participate or were lost-to-follow up. Unfortunately, we had to exclude these patients from the analysis which might have resulted in potential selection bias. This might have underestimated the current results as patients who refused to participate could be more severely ill due to disease progression. However, comparison of baseline characteristics among patients with and without missing postoperative WHODAS 2.0 did not show any differences (data not shown).

To conclude, no statistically significant differences were found on the association between PMI phenotypes and disability-free survival probably due the low number of patients in these groups. Clinical relevant differences were found for change in WHODAS 2.0 in patients with MI and patient with non-MI events with PMI. Isolated PMI was not associated with long term disability. Early recognition and subsequent management of cardiac and non-cardiac complications in patients at high risk might benefit disability free survival on the long-term. 


\section{REFERENCES}

1. Rose J, Weiser TG, Hider P, Wilson L, Gruen RL, Bickler SW. Estimated need for surgery worldwide based on prevalence of diseases: a modelling strategy for the WHO Global Health Estimate. Lancet Glob Health. 2015;3 Suppl 2:S13-20.

2. Weiser TG, Haynes AB, Molina G, Lipsitz SR, Esquivel MM, Uribe-Leitz T, et al. Estimate of the global volume of surgery in 2012: an assessment supporting improved health outcomes. Lancet. 2015;385 Suppl 2:S11.

3. Global patient outcomes after elective surgery: prospective cohort study in 27 low-, middleand high-income countries. Br J Anaesth. 2016;117(5):601-9.

4. van Waes JAR, Grobben RB, Nathoe HM, Kemperman H, de Borst GJ, Peelen LM, et al. OneYear Mortality, Causes of Death, and Cardiac Interventions in Patients with Postoperative Myocardial Injury. Anesthesia and Analgesia. 2016;123(1):29-37.

5. van Waes JAR, Nathoe HM, de Graaff JC, Kemperman H, de Borst GJ, Peelen LM, et al. Myocardial Injury After Noncardiac Surgery and its Association With Short-Term Mortality. Circulation. 2013;127(23):2264-71.

6. Beattie WS, Karkouti K, Tait G, Steel A, Yip P, McCluskey S, et al. Use of clinically based troponin underestimates the cardiac injury in non-cardiac surgery: a single-centre cohort study in 51,701 consecutive patients. Can J Anaesth. 2012;59(11):1013-22.

7. Devereaux PJ, Biccard BM, Sigamani A, Xavier D, Chan MTV, Srinathan SK, et al. Association of Postoperative High-Sensitivity Troponin Levels With Myocardial Injury and 30-Day Mortality Among Patients Undergoing Noncardiac Surgery. Jama. 2017;317(16):1642-51.

8. Devereaux PJ, Chan MTV, Alonso-Coello P, Walsh M, Berwanger O, Villar JC, et al. Association between postoperative troponin levels and 30-day mortality among patients undergoing noncardiac surgery. JAMA - Journal of the American Medical Association. 2012;307(21):2295304.

9. Beattie WS, Wijeysundera DN, Chan MTV, Peyton PJ, Leslie K, Paech MJ, et al. Survival After Isolated Post-Operative Troponin Elevation. J Am Coll Cardiol. 2017;70(7):907-8.

10. Beattie WS, Wijeysundera DN, Chan MTV, Peyton PJ, Leslie K, Paech MJ, et al. Implication of Major Adverse Postoperative Events and Myocardial Injury on Disability and Survival: A Planned Subanalysis of the ENIGMA-II Trial. Anesth Analg. 2018;127(5):1118-26.

11. Mackey WC, Fleisher LA, Haider S, Sheikh S, Cappelleri JC, Lee WC, et al. Perioperative myocardial ischemic injury in high-risk vascular surgery patients: incidence and clinical significance in a prospective clinical trial. J Vasc Surg. 2006;43(3):533-8.

12. Thygesen K, Alpert JS, Jaffe AS, Chaitman BR, Bax JJ, Morrow DA, et al. Fourth Universal Definition of Myocardial Infarction (2018). Circulation. 2018;138(20):e618-e51.

13. Kristensen SD, Knuuti J, Saraste A, Anker S, Botker HE, De Hert S, et al. 2014 ESC/ESA Guidelines on non-cardiac surgery: cardiovascular assessment and management: The Joint Task Force on non-cardiac surgery: cardiovascular assessment and management of the European Society of Cardiology (ESC) and the European Society of Anaesthesiology (ESA). Eur J Anaesthesiol. 2014;31(10):517-73.

14. Shulman MA, Myles PS, Chan MT, Mcllroy DR, Wallace S, Ponsford J. Measurement of disability-free survival after surgery. Anesthesiology. 2015;122(3):524-36. 
15. Ustun TB, Kostanjesek N, Chatterji S, Rehm J, World Health O. Measuring health and disability : manual for WHO Disability Assessment Schedule (WHODAS 2.0) / edited by T.B. Üstün, N. Kostanjsek, S. Chatterji, J.Rehm. Geneva: World Health Organization; 2010.

16. Lee TH, Marcantonio ER, Mangione CM, Thomas EJ, Polanczyk CA, Cook EF, et al. Derivation and prospective validation of a simple index for prediction of cardiac risk of major noncardiac surgery. Circulation. 1999;100(10):1043-9.

17. Mehta RL, Kellum JA, Shah SV, Molitoris BA, Ronco C, Warnock DG, et al. Acute Kidney Injury Network: report of an initiative to improve outcomes in acute kidney injury. Crit Care. 2007;11(2):R31.

18. Noordzij PG, van Geffen O, Dijkstra IM, Boerma D, Meinders AJ, Rettig TCD, et al. High-sensitive cardiac troponin $T$ measurements in prediction of non-cardiac complications after major abdominal surgery. British Journal of Anaesthesia. 2015;114(6):909-18.

19. Fleisher LA, Fleischmann KE, Auerbach AD, Barnason SA, Beckman JA, Bozkurt B, et al. 2014 ACC/AHA guideline on perioperative cardiovascular evaluation and management of patients undergoing noncardiac surgery: a report of the American College of Cardiology/American Heart Association Task Force on Practice Guidelines. Circulation. 2014;130(24):e278-333.

20. Myles PS. More than just morbidity and mortality - quality of recovery and long-term functional recovery after surgery. Anaesthesia. 2020;75 Suppl 1:e143-e50.

21. Moonesinghe SR, Jackson AIR, Boney O, Stevenson N, Chan MTV, Cook TM, et al. Systematic review and consensus definitions for the Standardised Endpoints in Perioperative Medicine initiative: patient-centred outcomes. Br J Anaesth. 2019;123(5):664-70.

22. Ladha KS, Wijeysundera DN. Role of patient-centred outcomes after hospital discharge: a state-of-the-art review. Anaesthesia. 2020;75 Suppl 1:e151-e7.

23. Joosten A, Delaporte A, Mortier J, Ickx B, Van Obbergh L, Vincent JL, et al. Long-term Impact of Crystalloid versus Colloid Solutions on Renal Function and Disability-free Survival after Major Abdominal Surgery. Anesthesiology. 2019;130(2):227-36.

24. Shulman MA, Cuthbertson BH, Wijeysundera DN, Pearse RM, Thompson B, Torres E, et al. Using the 6-minute walk test to predict disability-free survival after major surgery. $\mathrm{Br} \mathrm{J}$ Anaesth. 2019;122(1):111-9.

25. Grobben RB, van Waes JAR, Leiner T, Peelen LM, de Borst GJ, Vogely HC, et al. Unexpected Cardiac Computed Tomography Findings in Patients With Postoperative Myocardial Injury. Anesth Analg. 2018;126(5):1462-8.

26. Landesberg G, Beattie WS, Mosseri M, Jaffe AS, Alpert JS. Perioperative Myocardial Infarction. Circulation. 2009;119(22):2936-44. 


\section{SUPPLEMENTAL MATERIAL}

Supplemental Table 1. Frequencies of each of the strata represented in the alluvial plot

\begin{tabular}{|c|c|c|c|}
\hline $\begin{array}{l}\text { Preoperative } \\
\text { disability status }\end{array}$ & PMI phenotype & $\begin{array}{c}\text { Postoperative disability } \\
\text { status }\end{array}$ & Frequency \\
\hline No disability & No complications & Disability free survival & $281(43.0)$ \\
\hline No disability & Isolated PMI & Disability free survival & $36(5.5)$ \\
\hline No disability & Myocardial infarction & Disability free survival & $12(1.8)$ \\
\hline No disability & Non-MI events with PMI & Disability free survival & $13(2.0)$ \\
\hline No disability & $\begin{array}{l}\text { Non-Ml events without } \\
\text { PMI }\end{array}$ & Disability free survival & $46(7.0)$ \\
\hline No disability & No complications & Moderate & 69 (10.6) \\
\hline No disability & Isolated PMI & Moderate & $10(1.5)$ \\
\hline No disability & Myocardial infarction & Moderate & $1(0.2)$ \\
\hline No disability & Non-MI events with PMI & Moderate & $5(0.8)$ \\
\hline No disability & $\begin{array}{l}\text { Non-Ml events without } \\
\text { PMI }\end{array}$ & Moderate & $15(2.3)$ \\
\hline No disability & No complications & Severe & $14(2.1)$ \\
\hline No disability & Isolated PMI & Severe & $5(0.8)$ \\
\hline No disability & Myocardial infarction & Severe & $2(0.3)$ \\
\hline No disability & Non-MI events with PMI & Severe & $6(0.9)$ \\
\hline No disability & $\begin{array}{l}\text { Non-Ml events without } \\
\text { PMI }\end{array}$ & Severe & $8(1.2)$ \\
\hline Moderate & No complications & Disability free survival & $47(7.2)$ \\
\hline Moderate & Isolated PMI & Disability free survival & $10(1.5)$ \\
\hline Moderate & Myocardial infarction & Disability free survival & $2(0.3)$ \\
\hline Moderate & Non-MI events with PMI & Disability free survival & $5(0.8)$ \\
\hline Moderate & $\begin{array}{l}\text { Non-MI events without } \\
\text { PMI }\end{array}$ & Disability free survival & $9(1.4)$ \\
\hline Moderate & No complications & Moderate & $8(1.2)$ \\
\hline Moderate & Isolated PMI & Moderate & $0(0.0)$ \\
\hline Moderate & Myocardial infarction & Moderate & $1(0.2)$ \\
\hline Moderate & Non-MI events with PMI & Moderate & $0(0.0)$ \\
\hline Moderate & $\begin{array}{l}\text { Non-Ml events without } \\
\text { PMI }\end{array}$ & Moderate & $3(0.5)$ \\
\hline Moderate & No complications & Severe & $9(1.4)$ \\
\hline
\end{tabular}


Supplemental Table 1. Frequencies of each of the strata represented in the alluvial plot

\begin{tabular}{|c|c|c|c|}
\hline $\begin{array}{l}\text { Preoperative } \\
\text { disability status }\end{array}$ & PMI phenotype & $\begin{array}{c}\text { Postoperative disability } \\
\text { status }\end{array}$ & Frequency \\
\hline Moderate & Isolated PMI & Severe & $2(0.3)$ \\
\hline Moderate & Myocardial infarction & Severe & $3(0.5)$ \\
\hline Moderate & Non-MI events with PMI & Severe & $0(0.0)$ \\
\hline Moderate & $\begin{array}{l}\text { Non-MI events without } \\
\text { PMI }\end{array}$ & Severe & $2(0.3)$ \\
\hline Severe & No complications & Disability free survival & $16(2.5)$ \\
\hline Severe & Isolated PMI & Disability free survival & $4(0.6)$ \\
\hline Severe & Myocardial infarction & Disability free survival & $1(0.2)$ \\
\hline Severe & Non-MI events with PMI & Disability free survival & $3(0.5)$ \\
\hline Severe & $\begin{array}{l}\text { Non-Ml events without } \\
\text { PMI }\end{array}$ & Disability free survival & $0(0.0)$ \\
\hline Severe & No complications & Moderate & $0(0.0)$ \\
\hline Severe & Isolated PMI & Moderate & $0(0.0)$ \\
\hline Severe & Myocardial infarction & Moderate & $0(0.0)$ \\
\hline Severe & Non-MI events with PMI & Moderate & $0(0.0)$ \\
\hline Severe & $\begin{array}{l}\text { Non-MI events without } \\
\text { PMI }\end{array}$ & Moderate & $0(0.0)$ \\
\hline Severe & No complications & Severe & $1(0.2)$ \\
\hline Severe & Isolated PMI & Severe & $1(0.2)$ \\
\hline Severe & Myocardial infarction & Severe & $2(0.3)$ \\
\hline Severe & Non-MI events with PMI & Severe & $1(0.2)$ \\
\hline Severe & $\begin{array}{c}\text { Non-Ml events without } \\
\text { PMI }\end{array}$ & Severe & $0(0.0)$ \\
\hline
\end{tabular}


Supplemental Table 2. Adjusted associations between PMI phenotypes and disability

\begin{tabular}{|c|c|c|c|c|c|c|}
\hline & \multicolumn{3}{|c|}{$\begin{array}{c}\text { No new disability free } \\
\text { survival }\end{array}$} & \multicolumn{3}{|c|}{$\begin{array}{l}\text { Change in WHODAS } 2.0 \\
\text { score }\end{array}$} \\
\hline & $\mathbf{R R}$ & $95 \% \mathrm{Cl}$ & P-value & $\beta$ & $95 \% \mathrm{Cl}$ & P-value \\
\hline No complications & ref & & & ref & & \\
\hline Myocardial infarction & 1.51 & $0.69-2.93$ & 0.259 & 14.76 & $5.34-24.18$ & 0.002 \\
\hline Non-MI complications with PMI & 1.56 & $0.80-2.77$ & 0.154 & 9.21 & $1.16-17.27$ & 0.025 \\
\hline $\begin{array}{l}\text { Non-Ml complications without } \\
\text { PMI }\end{array}$ & 1.50 & $0.96-2.26$ & 0.061 & 7.06 & $1.76-12.36$ & 0.009 \\
\hline Isolated troponin & 1.08 & $0.62-1.76$ & 0.781 & 1.18 & $-4.67-7.03$ & 0.693 \\
\hline $\begin{array}{l}\text { Preoperative WHODAS } 2.0 \\
\text { score }\end{array}$ & 1.01 & $1.00-1.01$ & 0.300 & -0.4 & $-0.52--0.28$ & $<0.001$ \\
\hline Age & 1.00 & $0.97-1.02$ & 0.841 & 0.06 & $-2.77-0.35$ & 0.651 \\
\hline Sex (females) & 1.25 & $0.90-1.72$ & 0.177 & 1.97 & $-1.84-5.63$ & 0.292 \\
\hline Malignancy & 1.23 & $0.83-1.85$ & 0.307 & 3.62 & $-0.85-8.09$ & 0.113 \\
\hline \multicolumn{7}{|l|}{ RCRI factors } \\
\hline High risk surgery & 1.39 & $0.61-4.02$ & 0.483 & 6.26 & $-2.77-15.3$ & 0.194 \\
\hline Ischemic heart disease & 1.36 & $0.87-2.05$ & 0.158 & 1.28 & $-3.92-6.48$ & 0.432 \\
\hline History of heart failure & 0.65 & $0.19-1.62$ & 0.409 & -4.24 & $-15.56-7.09$ & 0.490 \\
\hline $\begin{array}{l}\text { History of cerebrovascular m } \\
\text { disease }\end{array}$ & 1.60 & $1.04-2.38$ & 0.026 & 9.28 & $3.77-14.79$ & 0.001 \\
\hline Insulin dependent diabetes & 1.16 & $0.71-1.84$ & 0.531 & 1.1 & $-4.54-6.74$ & 0.546 \\
\hline Elevated creatinine & 0.89 & $0.41-1.75$ & 0.763 & -1.23 & $-9.33-6.87$ & 0.852 \\
\hline \multicolumn{7}{|l|}{ Surgical specialty } \\
\hline ENT and dental & ref & & & ref & & \\
\hline General & 0.94 & $0.30-2.44$ & 0.905 & -2.44 & $-12.69-7.81$ & 0.641 \\
\hline Gynaecology and urology & 0.86 & $0.27-2.27$ & 0.778 & -6.64 & $-16.95-3.68$ & 0.208 \\
\hline Neurosurgery & 1.82 & $0.95-3.43$ & 0.067 & 3.52 & $-4.28-11.32$ & 0.377 \\
\hline Orthopaedics & 1.24 & $0.61-2.50$ & 0.555 & 1.24 & $-6.32-8.79$ & 0.749 \\
\hline Thoracic & 0.80 & $0.22-2.47$ & 0.710 & -3.96 & $-16.25-8.33$ & 0.528 \\
\hline Vascular & 0.96 & $0.29-2.65$ & 0.943 & -6.06 & $-17.06-4.95$ & 0.281 \\
\hline
\end{tabular}

* The percentages reported are the number of patient in that strata divided by the total number of included patients, i.e. 653 . The number of patients with no preoperative disability, moderate and severe disability were 523 (80\%), 101 (16\%) and 29 (4\%), respectively.

* MI: myocardial infarction, non-MI events: in-hospital complications except myocardial infarction; PMI: postoperative myocardial injury; WHODAS: world health organization disability assessment score; RCRI: revised cardiac risk index; ENT: ear, nose \& throat; ACE-inhibitor: angiotensin converting enzyme inhibitor; ARB: Angiotensin II receptor blocker; OR: odds ratio; Cl: confidence interval 


The aim of surgery is to cure disease or to relieve symptoms, thereby increasing life expectancy and improving quality of life. The perioperative period entails the period from the pre-anaesthesia assessment to hospital discharge, and may also include the recovery phase at home after discharge. Perioperative care includes individual patient risk assessment, optimizing preoperative status, and managing intra- and postoperative care, in order to avoid patient discomfort, to mitigate complications, and to improve recovery.

Research in this field is challenging. Such challenges exist in for example the large number of repeated measurements during hospital admission or even only during surgery alone, but also in the number and variety of events that may occur, which each could affect the patient's prognosis. Another challenge is the heterogeneity of the patient population that enters the operating room. The latter can be explained by the broad spectrum of surgical specialties for which a patient could undergo anaesthesia, but is also due to the variation in patient characteristics such as age, sex or comorbidities among these surgical patients. In this thesis, some of these methodologically challenging aspects were investigated. Throughout the chapters of the thesis the following lessons were learned:

In anaesthesia literature, there is considerable variation in methods used to express the severity of $\mathrm{IOH}$ exposure. Clear reporting guidance for research purposes is needed to improve reproducibility and comparability among studies reporting on $\mathrm{IOH}$. (Chapter 2) Different methods of modelling $\mathrm{IOH}$ yielded varying effects estimates with highly different levels of significance. This indicates that the method to model $\mathrm{OOH}$ strongly influences the magnitude of the association that is found. (Chapter $\mathbf{3}$ )

A large number of biomarkers and prediction models exist that were added or compared to the RCRI to improve the prediction of postoperative outcomes in patients undergoing non-cardiac surgery. The identified data were not sufficient to conclude that any of the biomarkers or prediction models shows improved predictive performance on top of the RCRI or as a replacement of the RCRI compared to the predictive accuracy of the RCRI alone. Pooling of results was not possible due to large heterogeneity in investigated biomarkers and prediction models, surgical population used, prediction horizons, the way the biomarker was studied and the varying composition of the outcome in especially MACE. (Chapter $\mathbf{4}$

\section{and 5).}

Implementation of routine standard consultation of a dedicated anaesthesiologist in patients with PMI resulted in an early detection of 59\% of all myocardial infarctions and involved a change in treatment in a considerable number of patients with postoperative troponin elevation. Whether this may improve patient outcomes on the long term remains to be elucidated. (Chapter $\mathbf{6}$ ) 
Postoperative complications were found to increase death and disability. No association was found for the different PMI phenotypes probably due the low number of included patients. Early recognition and management of cardiac and non-cardiac complications in patients at high risk might benefit disability free survival on the long-term. (Chapter $\mathbf{7}$ )

The sections below comprise a reflection of these studies in a broader context. Throughout this general discussion, two different patients are described as a clinical case example (Box 1).

\section{Box 1 Clinical case examples}

Patient A: A 75-year old patient with well-regulated hypertension and a history of myocardial infarction undergoes elective hip replacement. He is chronically treated with a beta blocker, an ACE-inhibitor, aspirin and a statin. Preoperatively, he has one RCRI factor (i.e. only history of ischemic heart disease, RCRI class 2), but an estimated poor functional capacity and a WHODAS 2.0 score of $60 \%$, meaning that he is severely disabled before surgery. The surgical procedure and postoperative course are both without any complications and the patient is discharged after 4 days. Six months after surgery, he has recovered well and the WHODAS score has improved to $30 \%$. This means that, according to the definition in the TEAMS study, the patient is classified as disability free survival after his hip surgery.

Patient B: A 68-year old patient with esophageal cancer and no other comorbidities undergoes elective esophagectomy. He does not use any medication chronically. Before surgery, he has RCRI class 2 (i.e. one RCRI factor; high risk surgery), good functional capacity and a WHODAS 2.0 score of $0 \%$, i.e. he has no preoperative disability. The surgery proceeds smoothly without any complications, but after surgery troponin elevation is detected and he suffers from pneumonia. He is discharged after 12 days. At six months after surgery, the cancer is metastasized to his brain and lungs and the patient is therefore admitted to a hospice. The WHODAS score is worsened to 63\%, meaning that the patient is severely disabled. He dies one month later.

\section{Intraoperative hypotension as a risk factor for postoperative adverse events}

During surgery, different vital parameters are measured repeatedly, including blood pressure. If the intraoperative blood pressure becomes too low (i.e. intraoperative hypotension, $1 \mathrm{OH}$ ), the anaesthesiologist could decide to treat this by administering for example vasopressors or extra fluids. $\mathrm{IOH}$ is associated with various postoperative in-hospital complications, including myocardial injury, acute kidney injury and death (1-3), and is hence an important topic of research within the domain of anaesthesia. 
However, the question remains 'which blood pressure is 'too low' and when is this a problem?' The recently published consensus statement on the definition of $\mathrm{IOH}$ states that blood pressure should be maintained above a certain threshold (i.e. systolic arterial pressure above $100 \mathrm{~mm} \mathrm{Hg}$ and a mean arterial pressure above $60-70 \mathrm{~mm} \mathrm{Hg}$ ), but it does not consider the severity of $\mathrm{IOH}$, e.g. in terms of duration and depth. (4) In Chapter $\mathbf{2}$ we have shown that in the anaesthesia literature a plethora of methods have been used to summarize the blood pressure values measured during the surgical procedure into a single measure to capture overall $1 \mathrm{OH}$ exposure. (5) Examples of such methods are the total duration below the threshold, lowest measured blood pressure or a combination of both reflecting the area under the threshold. Subsequently, in Chapter $\mathbf{3}$ we investigated whether associations between $\mathrm{IOH}$ and outcomes differ depending on the method used to express $1 \mathrm{OH}$ exposure. (6) We found that based on the modelling technique used, effect estimates for the association between $\mathrm{IOH}$ and outcomes varied considerably, which may lead to different conclusions and thereby hamper comparison of studies investigating the same association. In addition, the severity of $\mathrm{IOH}$ is also dependent on blood pressure management by anaesthesiologists. However, treatment to resolve $\mathrm{IOH}$ is most commonly not incorporated in research on $\mathrm{IOH}$ (Chapter 2).

One could argue that the differences in effect estimates we found are relatively small. This is potentially a reflection of the fact that the occurrence of postoperative myocardial injury, and other postoperative adverse events, is a multifactorial process. Intraoperative factors such as blood loss, surgical manipulation and duration of surgery are related to $\mathrm{IOH}$ in a dose-dependent manner. (3, 7-10) Longer surgical durations with extensive blood loss will likely result in more severe $\mathrm{IOH}$ compared to procedures with a shorter duration and no blood loss. Furthermore, preoperative patient characteristics including age, sex and comorbidities are also related to both the occurrence of $\mathrm{IOH}$ and in-hospital complications. (3, 7-10) Such factors but perhaps also other unmeasured factors are commonly more strongly related with adverse outcomes than $\mathrm{IOH} .(2,3)$ Furthermore, the incidence of $\mathrm{IOH}$ also depends on patient characteristics, as the anaesthesiologist may in practice adapt the threshold for intraoperative blood pressures based on those patient characteristics. For example, intraoperative blood pressures of patient A with preoperative comorbidities and use of anti-hypertensive drugs may have been strictly regulated as the use of anti-hypertensives such as angiotensin converting enzyme inhibitors (ACE-inhibitors) and beta blockers has been found to increase the incidence of post-induction hypotension. (1113) As a result, the probability of $\mathrm{IOH}$ might be lower for patient $A$ when compared to patient $B$.

Currently, research on $\mathrm{IOH}$ is of particular interest as it is one of the few intraoperative risk factors for adverse outcomes that can potentially be treated. Several studies aimed to avoid IOH using critical alerts, $(14,15)$ goal-directed hemodynamic therapy (16-18) or higher target intraoperative blood pressure (19) to reduce clinical outcomes. Thus far, no research has been published on 
blood pressure management to prevent $\mathrm{IOH}$ in order to improve patient centred outcomes in a broader patient population. The hypothesis is that more strict management of blood pressure will result in a reduction in the incidence of hypotension and in a subsequent reduction in functional disability of patients after surgery. Several international research groups are currently designing studies or recruiting patients to fill this research gap. (NCT03629418, and two studies granted by Netherlands Organisation for Health Research and Development in the start-up phase.)

\section{Risk stratification of the high risk patient}

As our population is aging and the incidence of multimorbidity is increasing, early identification of patients at high risk for complications is desirable to direct healthcare towards the most vulnerable patients. (20) Risk stratification, most commonly performed during the pre-anaesthesia assessment, has become a valuable tool for the development of individualized care planning. In risk stratification, prediction models and routine measurements of biomarkers are increasingly used. Multiple prognostic models have been developed for the prediction of postoperative outcomes. $(21,22)$ One of the most commonly used prediction models in clinical practice is the Revised Cardiac Risk Index (RCRI). The RCRI estimates the probability of in-hospital major adverse cardiac events (MACE) with moderate discriminative ability. $(23,24)$ Several authors aimed to improve the performance of the RCRI. (25-29) Chapter $\mathbf{4}$ and $\mathbf{5}$ provides a systematic review to identify studies that added biomarkers to the RCRI to increase the predictive accuracy, and studies that compared the predictive performance of biomarkers and other prediction models to the RCRI. In 84 included articles, we identified 56 unique biomarkers that were added to the RCRI to improve risk prediction. In addition, we found 47 biomarkers and 44 prediction models of which the predictive performance was compared to the RCRI. Due to large heterogeneity in investigated biomarkers and prediction models, surgical population used, prediction horizons, the scale how the biomarker was studied (i.e. continuous, dichotomous or categorical) and the varying composition of the outcome in especially MACE, pooling of the results was not possible (Chapter 5). As further research is recommended, several aspects should be considered.

First, to be useful for clinicians and to improve patient outcomes and consequently to increase cost-effectiveness, a prognostic model should not only provide validated and accurate predictions but also take health care resources into account. (30) Accordingly, focus should be on biomarkers that are low in costs, have low measurement invasiveness, have a short measuring time, and can be easily used to calculate predictions. (30) Second, research on single biomarkers for the prediction of outcomes should be avoided as clinical decisions are based on information from multiple factors, making predictions of single markers less relevant. $(31,32)$ Third, there should be consensus on the definition of outcomes including its composites for research purposes to be able to compare studies reporting similar predictions. For example, MACE was used as the primary outcome in approximately half of the included articles but almost all used another 
definition (Chapter 5). In an effort to improve standardized outcomes, the 'Standardized Endpoints for Perioperative Medicine' (StEP) Group was established with the aim to arrive at a set of standardized endpoints in perioperative medicine through a consensus process. (33-38) A standardized definition on cardiovascular endpoints has not been published yet. Fourth, as the reporting of prediction performance measures and the predictor definitions were poor, one should adhere to the "Transparent reporting of a multivariable prediction model for individual prognosis or diagnosis" (TRIPOD) guideline to be able to judge the validity and applicability of the studied prediction models. (39) Fifth, the RCRI does not predict well in patients undergoing vascular surgery, but provides moderate predictions for the non-cardiac nonvascular surgical population. (24) This means that addition of a particular biomarker to the RCRI could be highly predictive in one subgroup but not in another. For our case examples, the estimated risk of MACE using the RCRI is similar for both patients, i.e. one RCRI factor (class 2) reflects a probability of $0.9 \%$. However, it could be possible that the probability of developing MACE for patient A could be improved by adding cardiac biomarkers such as troponin or NT-proBNP to the model, whereas for patient B for example functional capacity may be more important. Finally, individual participant data (IPD) meta-analyses may be useful to investigate the added value of a particular biomarker. As studying the added predictive ability of a biomarker to the RCRI is commonly not part of the primary aim of the study, performing an IPD meta-analysis increases statistical power and is able to identify sources of variation within and across studies. (40)

\section{Troponin as a marker for adversity}

Perioperative routine surveillance of troponin is recommended by several guidelines. (41-43) The Canadian guideline on perioperative cardiac risk assessment and management advises to measure troponin daily in "patients with an elevated NT-proBNP/BNP measurement before surgery or in those who have a RCRI score $\geq 1$, age 45-64 years with significant cardiovascular disease, or age 65 years or older" (41), while the European guideline advises to measure troponin in "patients with METS $\leq 4$ or with a revised cardiac risk index value $>1$ for vascular surgery and $>2$ for non-vascular surgery" (43) and the 'Fourth Definition of Myocardial Infarction' expert consensus document suggests to measure troponin in "high risk" patients, but does not define this 'high risk'. (42) Based on these criteria, both patient A and B would apply to troponin monitoring using the recommendations of the Canadian guideline, while only patient A would apply according to the European guideline. For the Fourth Definition of Myocardial Infarction recommendations, this is even not clear. In the UMC Utrecht, troponin is measured in all patients aged $\geq 60$ years undergoing non-cardiac surgery with at least one overnight hospital stay.

Previously, we showed that elevated troponins were associated with 30-day (44) and one year mortality (45) although the cause of death was most commonly not due to cardiac events, at least in patients in whom the cause of death was known. In patients with postoperative myocardial 
injury (i.e. troponin elevation, PMI), only less than half of them were consulted by a cardiologist. (45) As a result, we implemented routine postoperative consultations by a dedicated anaesthesia team in patients with PMI. These consultations resulted in an early detection of $59 \%$ of myocardial infarctions, treatment was changed in terms of initiation of medication and blood transfusions in a considerable number of patients, and one out of four patients were referred to the cardiac outpatient clinic (Chapter 6). Unfortunately, we were not able to investigate whether this improves patient outcomes on the long term because follow up information was often missing as most patients were followed-up in their local hospital. In most patients, no clear cause of PMI was identified making treatment options difficult. Several studies aimed to prevent PMI by pharmacological suppression of the surgical stress response but this has not proven beneficial yet. (46) Recently, one large randomized controlled trial was undertaken in an attempt to treat patients with PMI using anticoagulants (i.e. dabigatran) and found reduced rates of vascular complications in the treated group with no statistical difference in the safety endpoint (i.e. the composite of lifethreatening, major, or critical organ bleeding). However, many patients with important bleeding problems, even requiring transfusion, were not included in this definition making these results less generalizable to real-world clinical practice. $(47,48)$ Altogether, we may conclude that PMI as detected by troponin surveillance has been associated with both short- and long-term mortality but that treatment options are currently limited.

PMI has been related to both cardiac disease, i.e. myocardial infarction, arrhythmia and heart failure, and non-cardiac disease subtypes including pulmonary embolism, acute kidney injury and sepsis. (Chapter 6 and 7, (45, 49, 50)) Two studies on PMI, including >15.000 patients each, considered injury only as caused by ischemia and excluded patients with PMI due non-ischemic postoperative complications (e.g. sepsis, pulmonary embolus, atrial fibrillation), although in both groups PMI was associated with 30-day mortality $(9,10)$ Beattie and colleagues, who did not exclude patients with PMI due to non-ischemic postoperative complications, showed that PMI with non-ischemic postoperative complications was associated with long-term mortality and disability in a dose-dependent manner. (50) In Chapter 7, similar PMI phenotypes as in the article of Beattie et al. (50) were compared in association with disability free survival at 6 months. We were not able to find differences among the PMI phenotypes and reduced disability free survival. The numbers of patients with myocardial infarction and non-MI complications with PMI were rather low (i.e. 24 and 33, respectively), resulting in wide confidence intervals and probably in non-significant results. As the study is currently still recruiting patients (i.e. the study size was determined at 1000 patients), no strong conclusions on the association between PMI phenotypes and disability free survival can be drawn yet from the results as presented in chapter $\mathbf{7}$. Furthermore, compared to the study of Beattie and colleagues (50) we used another tool for measuring disability free survival, and follow-up was performed at six months instead of one year. 
PMI should be considered as a marker of adversity and accordingly, health care resources should go to these patients at highest risk for complications and reduced disability free survival on the long term (Chapter $\mathbf{6}$ and $\mathbf{7}$ ). Early identification of complications by means of troponin surveillance might prevent morbidity and mortality on the long term and thereby decreasing costs. $(51,52)$ More severe complications result in prolonged hospitalization and thereby increasing health care costs. $(51,53)$ Selection of patients at high risk could be done by using, for example, prediction models such as the RCRI (23) or the NSQIP MICA (54) or by selection of patients who might benefit from troponin monitoring. Currently, such selection criteria are not available based on clinical data yet. This strategy could reduce costs as troponin in will not be measured in patients at low risk patients and health-care resources could be directed towards patients in need the most.

To get back to our case examples, patient B had an elevated troponin during hospital admission and was therefore consulted by the dedicated anaesthesiologist. An ECG was made, but no signs of ischemia were observed. On postoperative day 4 he developed cough and fever, and pneumonia was diagnosed. He was treated with antibiotics and was discharged a week later. Unfortunately, patient B worsened in his disability score due to disease progression and its effect on his emotional and physical well-being. He was classified as severely disabled. In patient A, no extra diagnostics were performed as his recovery during admission was as expected. At 6 months, he was mobile again resulting in more social interaction and an improved disability score meaning that he reached disability free survival.

Patient centred outcomes for research purposes have gained more importance compared to more traditional outcomes. Examples of traditional outcomes are medically important events including death, myocardial infarction, stroke and sepsis, but also length of hospital stay and reoperations. Such outcomes are especially important for treating physicians and health care insurers. (37, $55,56)$ However, outcomes important for patients are rather related to quality of life, functional status and life-impact measures (i.e. discharge to a nursing home). (37) Dependent on someone's position in the care process, an anaesthesiologist especially might focus on outcomes occurring intraoperatively or during hospital admission. A surgeon shares interest in similar outcomes, but likely may want to also include events occurring in the early recovery at home after discharge. A patient however focusses more on the long-term effects of surgery. For our case examples, both the anaesthesiologist and the surgeon agree that hospital admission for both patient $A$ and $B$ went smoothly, but patient B would argue whether this treatment strategy was a success as he became severely disabled 6 months after surgery. Therefore, focus should not only be on survival but also in the quality of that survival in terms of level of independence and function (i.e. work, self-care, engaging social activities and emotional well-being). (56) 
For making decisions on treatment strategies which could include surgery, the patient together with his treating physician aims for an improvement in quality of life, disability free survival, disease free survival or all mentioned before. However, such outcomes could differ depending on the diagnosis for which a patient undergoes surgery. For example, for patient A who undergoes hip replacement the most important aim is to improve disabilities and quality of life, whereas for patient $B$ who is diagnosed with cancer increasing life expectancy may be more important. Accordingly, comparing these different types of patients in a heterogeneous surgical population is complex. Patients undergoing surgery for cancer treatment most commonly do not experience any disabilities before surgery but might become disabled due to e.g. disease progression and side effects of chemotherapy (i.e. increase in disability score), whereas patients undergoing orthopaedic surgery are commonly disabled before surgery but improve in their disability score. For research purposes, including these patients with opposite trends in disability score in the same analysis might result in no difference in disability score for the whole population, while in fact this might not be the case. One solution would be to perform a subgroup analysis among surgical patients with and without cancer to explore differences between both groups. Another possibility would be to change the follow-up period to a shorter period, such as 3 months as the worsened disability score in patient B was probably not related to the procedure but due to disease progression.

\section{From diagnosis to hospital admission to full recovery}

At the diagnosis of a particular disease or health condition, a physician decides whether and what treatment strategy should be initiated for this individual patient as he aims for curing disease or relieving distressing symptoms, which hopefully results in disease free survival, disability free survival or both. Along this trajectory from diagnosis to full recovery, the risk of disease free survival and disability free survival are both likely to change. For example, at the time of diagnosis the chance of disability free survival for both patient $A$ and $B$ is considered to improve if the surgery is performed, despite the risk of intraoperative and postoperative complications which are both adversely associated with patient centred outcomes. Fortunately, for both patient A and $B$ the procedure itself was without complications but patient B suffered from postoperative troponin elevation and developed pneumonia during hospital admission. As a result, the probability of disability free survival on the long term changed during the postoperative phase. After discharge, patient A has fully recovered with disability free survival, but in patient B, the cancer has progressed and thereby reduced the probability of disability free survival.

Altogether, the probability for a particular outcome is not fixed and should be interpreted as dynamic. Accordingly, treatment decisions should be based on these dynamic risks. Further research is needed to be able to make such dynamic individualized predictions readily available in clinical practice along the course of treatment using statistical techniques such as multi-state transition models $(57,58)$ and machine learning. $(59,60)$ 


\section{REFERENCES}

1. Wesselink EM, Kappen TH, Torn HM, Slooter AJC, van Klei WA. Intraoperative hypotension and the risk of postoperative adverse outcomes: a systematic review. Br J Anaesth. 2018;121(4):706-21.

2. Salmasi V, Maheshwari K, Yang D, Mascha EJ, Singh A, Sessler DI, et al. Relationship between Intraoperative Hypotension, Defined by Either Reduction from Baseline or Absolute Thresholds, and Acute Kidney and Myocardial Injury after Noncardiac Surgery: A Retrospective Cohort Analysis. Anesthesiology. 2017;126(1):47-65.

3. van Waes JAR, van Klei WA, Wijeysundera DN, van Wolfswinkel L, Lindsay TF, Beattie WS. Association between Intraoperative Hypotension and Myocardial Injury after Vascular Surgery. Anesthesiology. 2016;124(1):35-44.

4. Sessler DI, Bloomstone JA, Aronson S, Berry C, Gan TJ, Kellum JA, et al. Perioperative Quality Initiative consensus statement on intraoperative blood pressure, risk and outcomes for elective surgery. Br J Anaesth. 2019;122(5):563-74.

5. Vernooij LM, van Klei WA, Moons KGM, van Waes JA, Peelen LM. Methods to express intraoperative hypotension exposure in the anaesthesia literature. Br J Anaesth. 2020.

6. Vernooij LM, van Klei WA, Machina M, Pasma W, Beattie WS, Peelen LM. Different methods of modelling intraoperative hypotension and their association with postoperative complications in patients undergoing non-cardiac surgery. British Journal of Anaesthesia. 2018;120(5):1080-9.

7. Reich DL, Hossain S, Krol M, Baez B, Patel P, Bernstein A, et al. Predictors of hypotension after induction of general anesthesia. Anesth Analg. 2005;101(3):622-8, table of contents.

8. Kawasaki S, Kiyohara C, Tokunaga S, Hoka S. Prediction of hemodynamic fluctuations after induction of general anesthesia using propofol in non-cardiac surgery: a retrospective cohort study. BMC Anesthesiol. 2018;18(1):167.

9. Devereaux PJ, Biccard BM, Sigamani A, Xavier D, Chan MTV, Srinathan SK, et al. Association of Postoperative High-Sensitivity Troponin Levels With Myocardial Injury and 30-Day Mortality Among Patients Undergoing Noncardiac Surgery. Jama. 2017;317(16):1642-51.

10. Devereaux PJ, Chan MTV, Alonso-Coello P, Walsh M, Berwanger O, Villar JC, et al. Association between postoperative troponin levels and 30-day mortality among patients undergoing noncardiac surgery. JAMA - Journal of the American Medical Association. 2012;307(21):2295304.

11. Kheterpal S, Khodaparast O, Shanks A, O'Reilly M, Tremper KK. Chronic angiotensinconverting enzyme inhibitor or angiotensin receptor blocker therapy combined with diuretic therapy is associated with increased episodes of hypotension in noncardiac surgery. $J$ Cardiothorac Vasc Anesth. 2008;22(2):180-6.

12. Hollmann C, Fernandes NL, Biccard BM. A Systematic Review of Outcomes Associated With Withholding or Continuing Angiotensin-Converting Enzyme Inhibitors and Angiotensin Receptor Blockers Before Noncardiac Surgery. Anesth Analg. 2018;127(3):678-87.

13. Blessberger H, Lewis SR, Pritchard MW, Fawcett LJ, Domanovits H, Schlager O, et al. Perioperative beta-blockers for preventing surgery-related mortality and morbidity in adults undergoing cardiac surgery. Cochrane Database Syst Rev. 2019;9:Cd013435. 
14. Panjasawatwong K, Sessler DI, Stapelfeldt WH, Mayers DB, Mascha EJ, Yang D, et al. A Randomized Trial of a Supplemental Alarm for Critically Low Systolic Blood Pressure. Anesth Analg. 2015;121(6):1500-7.

15. McCormick PJ, Levin MA, Lin HM, Sessler DI, Reich DL. Effectiveness of an Electronic Alert for Hypotension and Low Bispectral Index on 90-day Postoperative Mortality: A Prospective, Randomized Trial. Anesthesiology. 2016;125(6):1113-20.

16. Sun Y, Chai F, Pan C, Romeiser JL, Gan TJ. Effect of perioperative goal-directed hemodynamic therapy on postoperative recovery following major abdominal surgery-a systematic review and meta-analysis of randomized controlled trials. Crit Care. 2017;21(1):141.

17. Chong MA, Wang Y, Berbenetz NM, McConachie I. Does goal-directed haemodynamic and fluid therapy improve peri-operative outcomes?: A systematic review and meta-analysis. Eur J Anaesthesiol. 2018;35(7):469-83.

18. Michard F, Giglio MT, Brienza N. Perioperative goal-directed therapy with uncalibrated pulse contour methods: impact on fluid management and postoperative outcome. Br J Anaesth. 2017;119(1):22-30.

19. Futier E, Lefrant JY, Guinot PG, Godet T, Lorne E, Cuvillon P, et al. Effect of Individualized vs Standard Blood Pressure Management Strategies on Postoperative Organ Dysfunction Among High-Risk Patients Undergoing Major Surgery: A Randomized Clinical Trial. Jama. 2017;318(14):1346-57.

20. Wolff JL, Starfield B, Anderson G. Prevalence, expenditures, and complications of multiple chronic conditions in the elderly. Arch Intern Med. 2002;162(20):2269-76.

21. Wright DE, Knuesel SJ, Nagarur A, Philpotts LL, Greenwald JL. Examining Risk: A Systematic Review of Perioperative Cardiac Risk Prediction Indices. Mayo Clin Proc. 2019;94(11):2277-90.

22. Bose S, Talmor D. Who is a high-risk surgical patient? Curr Opin Crit Care. 2018;24(6):547-53.

23. Lee TH, Marcantonio ER, Mangione CM, Thomas EJ, Polanczyk CA, Cook EF, et al. Derivation and prospective validation of a simple index for prediction of cardiac risk of major noncardiac surgery. Circulation. 1999;100(10):1043-9.

24. Ford MK, Beattie WS, Wijeysundera DN. Systematic Review: Prediction of Perioperative Cardiac Complications and Mortality by the Revised Cardiac Risk Index. Annals of Internal Medicine. 2010;152(1):26-W7.

25. Kopec M, Duma A, Helwani MA, Brown J, Brown F, Gage BF, et al. Improving Prediction of Postoperative Myocardial Infarction With High-Sensitivity Cardiac Troponin T and NTproBNP. Anesthesia and Analgesia. 2017;124(2):398-405.

26. Choi JH, Cho DK, Song YB, Hahn JY, Choi S, Gwon HC, et al. Preoperative NT-proBNP and CRP predict perioperative major cardiovascular events in non-cardiac surgery. Heart. 2010;96(1):56-62.

27. Scrutinio D, Guido G, Guida P, Passantino A, Angiletta D, Santoro D, et al. Combined Use of High-sensitivity C-Reactive Protein and N-Terminal Pro-B-type Natriuretic Peptide for Risk Stratification of Vascular Surgery Patients. Annals of Vascular Surgery. 2014;28(6):1522-9.

28. Gillmann H-J, Meinders A, Grosshennig A, Larmann J, Buente C, Calmer S, et al. Perioperative Levels and Changes of High-Sensitivity Troponin T Are Associated With Cardiovascular Events in Vascular Surgery Patients. Critical Care Medicine. 2014;42(6):1498-506. 
29. Wijeysundera DN, Pearse RM, Shulman MA, Abbott TEF, Torres E, Ambosta A, et al. Assessment of functional capacity before major non-cardiac surgery: an international, prospective cohort study. Lancet. 2018;391(10140):2631-40.

30. Steyerberg EW, Moons KG, van der Windt DA, Hayden JA, Perel P, Schroter S, et al. Prognosis Research Strategy (PROGRESS) 3: prognostic model research. PLoS Med. 2013;10(2):e1001381.

31. Moons KG, van Es GA, Michel BC, Buller HR, Habbema JD, Grobbee DE. Redundancy of single diagnostic test evaluation. Epidemiology. 1999;10(3):276-81.

32. Linnet K, Bossuyt PM, Moons KG, Reitsma JB. Quantifying the accuracy of a diagnostic test or marker. Clin Chem. 2012;58(9):1292-301.

33. Myles PS, Grocott MP, Boney O, Moonesinghe SR. Standardizing end points in perioperative trials: towards a core and extended outcome set. Br J Anaesth. 2016;116(5):586-9.

34. Barnes J, Hunter J, Harris S, Shankar-Hari M, Diouf E, Jammer I, et al. Systematic review and consensus definitions for the Standardised Endpoints in Perioperative Medicine (StEP) initiative: infection and sepsis. Br J Anaesth. 2019;122(4):500-8.

35. Buggy DJ, Freeman J, Johnson MZ, Leslie K, Riedel B, Sessler DI, et al. Systematic review and consensus definitions for standardised endpoints in perioperative medicine: postoperative cancer outcomes. Br J Anaesth. 2018;121(1):38-44.

36. Haller G, Bampoe S, Cook T, Fleisher LA, Grocott MPW, Neuman M, et al. Systematic review and consensus definitions for the Standardised Endpoints in Perioperative Medicine initiative: clinical indicators. Br J Anaesth. 2019;123(2):228-37.

37. Moonesinghe SR, Jackson AIR, Boney O, Stevenson N, Chan MTV, Cook TM, et al. Systematic review and consensus definitions for the Standardised Endpoints in Perioperative Medicine initiative: patient-centred outcomes. Br J Anaesth. 2019;123(5):664-70.

38. Myles PS, Boney O, Botti M, Cyna AM, Gan TJ, Jensen MP, et al. Systematic review and consensus definitions for the Standardised Endpoints in Perioperative Medicine (StEP) initiative: patient comfort. Br J Anaesth. 2018;120(4):705-11.

39. Collins GS, Reitsma JB, Altman DG, Moons KG. Transparent Reporting of a multivariable prediction model for Individual Prognosis Or Diagnosis (TRIPOD). Ann Intern Med. 2015;162(10):735-6.

40. Debray TPA, de Jong VMT, Moons KGM, Riley RD. Evidence synthesis in prognosis research. Diagn Progn Res. 2019;3:13.

41. Duceppe E, Parlow J, MacDonald P, Lyons K, McMullen M, Srinathan S, et al. Canadian Cardiovascular Society Guidelines on Perioperative Cardiac Risk Assessment and Management for Patients Who Undergo Noncardiac Surgery. Canadian Journal of Cardiology. 2017;33(1):17-32.

42. Thygesen K, Alpert JS, Jaffe AS, Chaitman BR, Bax JJ, Morrow DA, et al. Fourth Universal Definition of Myocardial Infarction (2018). Circulation. 2018;138(20):e618-e51.

43. Fleisher LA, Fleischmann KE, Auerbach AD, Barnason SA, Beckman JA, Bozkurt B, et al. 2014 ACC/AHA guideline on perioperative cardiovascular evaluation and management of patients undergoing noncardiac surgery: a report of the American College of Cardiology/American Heart Association Task Force on Practice Guidelines. Circulation. 2014;130(24):e278-333.

44. van Waes JAR, Nathoe HM, de Graaff JC, Kemperman H, de Borst GJ, Peelen LM, et al. Myocardial Injury After Noncardiac Surgery and its Association With Short-Term Mortality. Circulation. 2013;127(23):2264-71. 
45. van Waes JAR, Grobben RB, Nathoe HM, Kemperman H, de Borst GJ, Peelen LM, et al. OneYear Mortality, Causes of Death, and Cardiac Interventions in Patients with Postoperative Myocardial Injury. Anesthesia and Analgesia. 2016;123(1):29-37.

46. Verbree-Willemsen L, Grobben RB, van Waes JA, Peelen LM, Nathoe HM, van Klei WA, et al. Causes and prevention of postoperative myocardial injury. Eur J Prev Cardiol. 2019;26(1):5967.

47. Devereaux PJ, Duceppe E, Guyatt G, Tandon V, Rodseth R, Biccard BM, et al. Dabigatran in patients with myocardial injury after non-cardiac surgery (MANAGE): an international, randomised, placebo-controlled trial. Lancet. 2018;391(10137):2325-34.

48. Adriaenssens T, Sinnaeve P. Direct oral anticoagulants for postoperative myocardial injury. Lancet. 2018;391(10137):2297-8.

49. Beattie WS, Wijeysundera DN, Chan MTV, Peyton PJ, Leslie K, Paech MJ, et al. Survival After Isolated Post-Operative Troponin Elevation. J Am Coll Cardiol. 2017;70(7):907-8.

50. Beattie WS, Wijeysundera DN, Chan MTV, Peyton PJ, Leslie K, Paech MJ, et al. Implication of Major Adverse Postoperative Events and Myocardial Injury on Disability and Survival: A Planned Subanalysis of the ENIGMA-II Trial. Anesth Analg. 2018;127(5):1118-26.

51. Patel AS, Bergman A, Moore BW, Haglund U. The economic burden of complications occurring in major surgical procedures: a systematic review. Appl Health Econ Health Policy. 2013;11(6):577-92.

52. Buse GL, Manns B, Lamy A, Guyatt G, Polanczyk CA, Chan MTV, et al. Troponin T monitoring to detect myocardial injury after noncardiac surgery: a cost-consequence analysis. Can J Surg. 2018;61(3):185-94.

53. Landais A, Morel M, Goldstein J, Loriau J, Fresnel A, Chevalier C, et al. Evaluation of financial burden following complications after major surgery in France: Potential return after perioperative goal-directed therapy. Anaesth Crit Care Pain Med. 2017;36(3):151-5.

54. Bilimoria KY, Liu Y, Paruch JL, Zhou L, Kmiecik TE, Ko CY, et al. Development and evaluation of the universal ACS NSQIP surgical risk calculator: a decision aid and informed consent tool for patients and surgeons. J Am Coll Surg. 2013;217(5):833-42.e1-3.

55. Myles PS. More than just morbidity and mortality - quality of recovery and long-term functional recovery after surgery. Anaesthesia. 2020;75 Suppl 1:e143-e50.

56. Ladha KS, Wijeysundera DN. Role of patient-centred outcomes after hospital discharge: a state-of-the-art review. Anaesthesia. 2020;75 Suppl 1:e151-e7.

57. Putter H, Fiocco M, Geskus RB. Tutorial in biostatistics: competing risks and multi-state models. Stat Med. 2007;26(11):2389-430.

58. Manzini G, Ettrich TJ, Kremer M, Kornmann M, Henne-Bruns D, Eikema DA, et al. Advantages of a multi-state approach in surgical research: how intermediate events and risk factor profile affect the prognosis of a patient with locally advanced rectal cancer. BMC Med Res Methodol. 2018;18(1):23.

59. Connor CW. Artificial Intelligence and Machine Learning in Anesthesiology. Anesthesiology. 2019;131(6):1346-59.

60. Fritz BA, Chen Y, Murray-Torres TM, Gregory S, Ben Abdallah A, Kronzer A, et al. Using machine learning techniques to develop forecasting algorithms for postoperative complications: protocol for a retrospective study. BMJ Open. 2018;8(4):e020124. 



\section{APPENDICES}

Summary

Nederlandse samenvatting

List of publications

Dankwoord

About the author 


\section{SUMMARY}

Every year, several million patients undergo surgery with the aim to cure disease or relieve symptoms thereby increasing life expectancy and improving quality of life. The perioperative period entails the period from the pre-anesthesia assessment to hospital discharge, and may also include the recovery phase at home after discharge. Research in the perioperative period is challenging due to e.g. the large number repeated measurements with multiple events that follow each other rapidly and the heterogeneous group of patients that enter the operating room. In this thesis, some of these methodologically challenging aspects were investigated.

Part I addresses how to summarize repeated measured intraoperative blood pressure measurements in research on hypotension. Hypotension refers to a blood pressure which is too low and is a common side effect of anaesthesia during surgery. It has been associated with adverse postoperative complications, including mortality, myocardial injury, stroke and acute kidney injury. Despite a recent consensus definition of intraoperative hypotension (IOH) for clinical purposes, there is currently no standardized methodology on how to incorporate severity of $\mathrm{IOH}$ in research. As there is considerable variation in the methods to express $\mathrm{IOH}$ exposure, we conducted a systematic review to investigate which methods have been used to express and analyse the magnitude of $\mathrm{IOH}$ in recent anaesthesia literature (Chapter 2). Screening of 1,282 titles and abstracts resulted in 237 included articles. The most frequently used category was Incidence (90\%) followed by Duration (28\%) and Lowest value (27\%). Incidence of hypotension $(72 \%)$ and Vasopressor administration (49\%) were the most commonly used methods. Reporting rates were higher when more complex methods were used. Standardized definitions of $\mathrm{IOH}$ including clear reporting guidance for research purposes is needed to improve reproducibility and comparability among studies. In Chapter 3, we examined whether using different methods to model $\mathrm{IOH}$ exposure affected the association with postoperative myocardial injury (PMI) and acute kidney injury (AKI). We included 10,432 patients aged $\geq 50$ years undergoing non-cardiac surgery. Twelve different methods to express the severity of $\mathrm{IOH}$ (representing presence, depth, duration and area under the threshold (AUT)) were applied to examine the association with PMI and AKI. Different methods to model $\mathrm{IOH}$ yielded effect estimates differing in size and statistical significance. Methods with the highest odds were absolute maximum fall in blood pressure (BP) and mean episode AUT (odds ratio (OR) with 99\% confidence interval (CI): 1.43 (1.15-1.77) and 1.69 (0.99-2.88), respectively, for the absolute mean arterial pressure $50 \mathrm{~mm} \mathrm{Hg}$ threshold). After standardization (i.e. comparing each of the methods on the same scale), the highest standardized ORs were obtained for depth related methods (OR with 99\% Cl: 1.12 (1.05-1.20)). No single method always yielded the highest effect estimate in every setting. This makes different studies on $\mathrm{IOH}$ less comparable and hampers clinical application of reported results. 
Part II investigates preoperative risk stratification of patients undergoing non-cardiac surgery at risk for postoperative cardiovascular complications. The Revised Cardiac Risk index (RCRI) is a predictive tool that estimates the probability of in-hospital major adverse cardiac events (MACE) in patients undergoing major non-cardiac surgery. Although the RCRI is commonly used in clinical practice, its predictive performance is only moderate. As a result, different biomarkers and other prediction models were added or compared to the RCRI to improve the predictive performance of this model. To investigate what biomarkers and prediction models have been added or compared to the RCRI and to quantify the predictive value of these biomarkers and prediction models to the RCRI to predict major adverse cardiac events (MACE), we conducted a systematic review (Chapter $\mathbf{4}$ and 5). We included studies among patients who underwent major non-cardiac surgery, reporting on the external validation of the original RCRI model alone and either the addition of one (of more) predictor(s) to the RCRI, the comparison of the predictive accuracy of (bio)marker to the RCRI or the comparison of the RCRI to other prediction models. We included 84 articles, of which 43 articles reported on the added value of predictors to the RCRI and 44 and 36 articles compared the predictive performance of the RCRI to biomarkers and other prediction models, respectively. However, we did not find added predictive value of biomarker(s) to the RCRI or other prediction models with better predictive performance compared to the RCRI alone to predict MACE in patients undergoing non-cardiac surgery. Further research is needed on the added predictive value of biomarkers to improve risk prediction using the RCRI. Preferably, focus should be on biomarker(s) that are low in costs, measurement duration should be short and not be invasiveness. Individual patient data meta-analyses in this research area might be beneficial to identify biomarkers with added value to the RCRI.

Routine postoperative cardiac biomarker surveillance is recommended by several guidelines to early identify patients with postoperative myocardial infarction. Postoperative myocardial infarction is often not diagnosed as its presentation is most often asymptomatic due to analgesic effects of anesthesia and distracting pain of the surgical wound. Postoperative myocardial injury, i.e. elevated cardiac troponins, is independently associated with morbidity and mortality in a dose dependent manner. Besides cardiac ischemia, release of troponin has also been related to other cardiac diseases, i.e. arrhythmia and heart failure, but also non-cardiac pathologies such as sepsis, pulmonary embolism, acute kidney injury and stroke. In the UMC Utrecht, troponin is routinely measured in all postoperative non-cardiac surgical patients $\geq 60$ years old with at least one overnight hospital stay. Part III focuses on the effect of postoperative myocardial injury on disability-free survival and health care resources during hospitalization. To conduct follow-up on patients with postoperative troponin elevation, we implemented a dedicated anesthesia team (Chapter 6). We hypothesized that these visits would facilitate early detection of complications. We included 811 patients of whom 509 (63\%) received a postoperative consultation by the 
anesthesiologist, which was higher compared to the consultation rates by a cardiologist (i.e. $41 \%$ ) before implementation. Anesthesiologists were involved in the early detection of $59 \%$ of all myocardial infarctions and in $12 \%$ of all complications. In $75 \%$ of patients, anesthesiologists ordered additional diagnostics, most frequently existing of electrocardiograms and additional cardiac enzyme testing. Additionally, change in treatment was advised, most often a medication change, in 16\% of patients and $23 \%$ were referred to the cardiac outpatient clinic for further followup after discharge. Improvement in patient outcomes remains to be elucidated as we were not able to obtain these follow-up as most were performed at local hospitals.

The independent effects of PMI phenotypes and on disability-free survival following noncardiac surgery were investigated in Chapter $\mathbf{7}$. The most common phenotype of postoperative myocardial injury s myocardial infarction, though PMI has also been related to other cardiac and non-cardiac pathologies. In this study, patients were stratified based on PMI and the occurrence of postoperative adverse events. This resulted in five groups, i.e. no adverse events, isolated PMI, myocardial infarction (MI), and complications with or without PMI but no myocardial infarction. The patient centered impact in terms of disability for these PMI phenotypes are largely unknown. This multicenter prospective cohort study included 653 patients ( $\geq 50$ years) undergoing elective major non-cardiac. PMI occurred in 125 (19\%) patients and 485 (74\%) had disability-free survival at 6 months. We were not able to find differences in the association between PMI phenotypes and disability-free survival. However, a clinically relevant change in disability score after surgery was found for patients with myocardial infarction and patients with non-MI events with PMI. Early recognition and management of cardiac and non-cardiac complications in patients at high risk might benefit disability-free survival on the long-term.

To conclude, this thesis has focused on different methodologically challenging aspects of research in the perioperative period, i.e. from preoperative assessment to surgery, to postoperative recovery in the hospital and at home. Future research should focus on the handling of a large number of measurements in terms of summarizing measures that could clearly reflect the whole period of interest or dynamic risk predictions as risk of a particular outcome could change along the perioperative period. Furthermore, more emphasis should be on selection of patients to direct health care resources to the most in need during both hospital admission and the recovery phase afterwards that also includes long term outcomes. 


\section{NEDERLANDSE SAMENVATTING}

Elkjaar worden er wereldwijd miljoenen patiënten geopereerd. Het ultieme doel van een operatie is om van ziekte te genezen of symptomen te verminderen om daarbij de levensverwachting te verhogen en de kwaliteit van leven te verbeteren. De perioperatieve periode is de periode rondom de operatie. Deze omvat onder andere het screenen van de patiënt voor de operatie, de operatie zelf, het ontslag uit het ziekenhuis en kan zelfs worden doorgetrokken naar de herstelfase die thuis plaatsvindt. Het uitvoeren van onderzoek tijdens deze perioperatieve periode is complex. Allereerst is de groep van patiënten die een operatie ondergaat buitengewoon heterogeen, van verschillen in de medische voorgeschiedenis tot de indicatie om te opereren. Daarnaast worden er grote hoeveelheden metingen uitgevoerd in een relatief korte periode, bijvoorbeeld de bloeddrukmetingen dat tijdens de operatie elke minuut plaatsvindt en de diverse bepalingen uit het bloed na de operatie. Ten slotte kunnen er bij patiënten in de perioperatieve periode ingrijpende gebeurtenissen zoals complicaties, optreden. In dit proefschrift zijn een aantal onderdelen van deze methodologisch uitdagende aspecten onderzocht.

Deel I van dit proefschrift richt zich op manieren om het grote aantal van herhaalde bloeddrukmetingen tijdens een operatie samen te vatten in onderzoek naar intra-operatieve hypotensie. Intra-operatieve hypotensie is een te lage bloeddruk tijdens de operatie. Het is gerelateerd aan het optreden van verschillende complicaties na de operaties zoals dood, een hartinfarct, een beroerte en acute nierschade. Aangezien intra-operatieve hypotensie vaak voorkomt en het tijdens de operatie potentieel vóórkomen kan worden, wordt hier veel onderzoek naar gedaan. Ondanks dat er nu een overeenstemming is over wat een té lage bloeddruk is, is er nog geen standaard methodologie om de ernst van intra-operatieve hypotensie vast te stellen en te gebruiken voor onderzoeksdoeleinden. Een standaard methodologie is belangrijk om verschillende onderzoeken naar intra-operatieve hypotensie met elkaar te kunnen vergelijken. Aangezien er veel variatie zit in deze methoden, hebben we in hoofdstuk $\mathbf{2}$ systematisch in de literatuur gezocht naar methoden die worden gebruikt om de ernst van intra-operatieve hypotensie samen te vatten voor onderzoeksdoeleinden binnen het anesthesiologische vakgebied. De zoekopdracht resulteerde in 1,282 artikelen, waarvan 237 artikelen minstens één methode beschreven om de ernst van intra-operatieve hypotensie samen te vatten. De gevonden methoden waren het vaakst gerelateerd aan de incidentie (90\%), ofwel "Heeft de patiënt minstens één keer tijdens de operatie een té lage bloeddruk gehad?". In 28\% van gevonden methoden werd de duur van de intra-operatieve hypotensie gebruikt als een weergave van ernst en in 27\% de laagst gemeten bloeddrukwaarde. Om verschillende artikelen met elkaar te kunnen vergelijken en vervolgens eventueel te kunnen reproduceren is het belangrijk dat de methoden goed beschreven worden. Slechts wanneer de methoden complex waren, werden de methoden vaker beschreven. In hoofdstuk $\mathbf{3}$ hebben we twaalf verschillende methoden geselecteerd om te onderzoeken of de sterkte van de associatie tussen intra-operatieve hypotensie met schade 
aan de hartspier en acute nierschade afhangt van de samenvattingsmaat die wordt gebruikt om de ernst van intra-operatieve hypotensie weer te geven. Voor dit onderzoek hebben we meer dan 10,000 patiënten van 50 jaar en ouder geselecteerd die een niet-hart gerelateerde operatie hebben ondergaan in het UMC Utrecht en Universtity Health Network Toronto, Canada. Drie voorbeelden van geselecteerde methoden waren het meten van de laagst gemeten bloeddrukwaarde, en de aanwezigheid en de totale duur van intra-operatieve hypotensie. Het bleek dat elk van de methoden om de ernst van intra-operatieve hypotensie samen te vatten geassocieerd was met complicaties na de operatie, waarbij de sterkte van de associatie verschillend was onder de twaalf methoden. Hierdoor zijn onderzoeken waarin verschillende methoden gebruikt worden om de ernst van intra-operatieve hypotensie uit te drukken niet goed met elkaar te vergelijken. Tevens bemoeilijkt dit de vertaalslag van de gevonden resultaten naar de kliniek.

Deel $\mathbf{2}$ focust zich op het voorspellen van het risico op het krijgen van hart gerelateerde complicaties bij patiënten die een niet-hart gerelateerde operatie ondergaan. De Revised Cardiac Risk Index (RCRI) is voorspeltool dat voor de operatie de kans het krijgen van een hart-gerelateerde complicatie na de operatie kan voorspellen bij patiënten die een operatie ondergaan maar niet aan het hart. Deze tool gebruikt informatie uit de medische voorgeschiedenis (bijvoorbeeld het gebruik van insuline, het doorgemaakt hebben van een hartinfarct, beroerte en/of TIA) om een voorspelling te kunnen maken. Ondanks dat deze tool in de klinische praktijk al veel wordt gebruikt, zijn de voorspellingen niet nauwkeurig. Om de nauwkeuringheid van de voorspellingen te vergroten hebben verschillende onderzoekers informatie van biomarkers toegevoegd aan de RCRI. Biomarkers zijn biologische metingen zoals bloedwaarden en beeldvormende informatie. Om te onderzoeken welke biomarkers er zijn toegevoegd aan de RCRI of vergeleken met de RCRI om de voorspelling van hart gerelateerde complicaties voor de operatie te kunnen verbeteren, hebben we systematisch in de literatuur gezocht (Hoofdstuk 4 en 5). We hebben artikelen geselecteerd van patiënten die een niet-hart gerelateerde operatie hebben ondergaan, waarbij de voorspellingen van de RCRI zelf werden vergeleken met de RCRI met toegevoegde biomarker(s) of alleen de biomarker(s) zonder RCRI. We hebben in totaal 84 artikelen geselecteerd waarvan 43 artikelen voorspellingen vergeleken van de RCRI ten opzichte van de RCRI plus biomarker(s) en 44 artikelen die de voorspellende waarde van alleen de biomarker(s) vergeleken met de RCRI. Ondanks de hoge diversiteit tussen onderzochte biomarkers, was geen enkele biomarker in staat om de voorspelling van hart-gerelateerde complicaties voor de operatie te kunnen verbeteren. Dit betekent dat er is meer onderzoek nodig is om de toegevoegde voorspellende waarde van biomarkers op de RCRI om risico inschatting op hart gerelateerde complicaties te kunnen verbeteren. Focus zou dan bij voorkeur gegeven moeten worden aan goedkope biomarkers met een snelle en niet-invasieve meetwijze. 
Een belangrijk biomarker die schade aan de hartspier kan identificeren is troponine. Deze biomarker komt vrij wanneer het hart tijdelijk te weinig zuurstof heeft ontvangen. Een verhoogd troponine kan wijzen op het doormaken van een hartinfarct na de operatie. Verschillende richtlijnen adviseren om na de operatie een biomarker te meten die schade aan de hartspier weergeeft om patiënten met een hartinfarct te kunnen identificeren. Het doormaken van een hartinfarct na een operatie wordt vaak niet opgemerkt omdat veel patiënten geen typische symptomen vertonen, zoals pijn op de borst. Dit komt doordat pijnklachten worden gemaskeerd door pijnmedicatie of door pijn bij de operatiewond. Naast de relatie van een verhoogd troponine en het optreden van een hartinfarct, is er een verband tussen een verhoogd troponine en het optreden van andere complicaties aan zowel het hart als andere organen. Voorbeelden zijn hartritmestoornissen, hartfalen, maar ook het optreden van een longembolie, een beroerte, acute nierschade en sepsis. Daarom wordt in het UMC Utrecht sinds 2011 bij alle patiënten van 60 jaar en ouder die een niet-hartgerelateerde operatie ondergaan dagelijks tot drie dagen na de operatie troponine gemeten. Om patiënten met een verhoogd troponine beter in de gaten te kunnen houden, is er een team van anesthesiologen die deze patiënten tijdens de ziekenhuisopname volgt om eerder complicaties te identificeren en eventueel behandeling aan te passen. Deel $\mathbf{3}$ van dit proefschrift onderzoekt de effecten van het optreden van schade aan de hartspier na de operatie op het gebruik van ziekenhuisresources en beperking in het dagelijks functioneren na zes maanden. In hoofdstuk $\mathbf{6}$ hebben we gekeken of er een effect is van een consultatie door de anesthesioloog bij patiënten met een verhoogd troponine op het vroegtijdig identificeren van complicaties en of dit resulteerde in een verhoogd gebruik van ziekenhuisresources. Voor dit onderzoek hebben we 811 patiënten met een verhoogd troponine na een niet-hart gerelateerde operatie geselecteerd. Bij 63\% van de patiënten heeft de anesthesioloog geconsulteerd. Consultatie door een anesthesioloog gebeurde vaker ten opzichte van de periode daarvoor waarin de cardioloog consulten uitvoerde (41\%). Daarnaast waren anesthesiologen betrokken bij de diagnose van 59\% van alle opgetreden hartinfarcten en in 12\% van alle opgetreden complicaties. Bij 75\% van de geconsulteerde patiënten werd extra diagnostiek uitgevoerd zoals het maken van een hartfilmpje (ECG) en het meten van extra hart-gerelateerde bloedwaarden. Een wijziging in behandeling geadviseerd (bijv. toedienen van een bloedtransfusie of wijziging in het gebruik van medicatie) werd in 16\% van de patiënten geadviseerd en $23 \%$ van de patiënten werd doorverwezen voor een polibezoek naar de cardioloog na ontslag uit het ziekenhuis. In hoofdstuk $\mathbf{7}$ is onderzocht of een verhoogd troponine in combinatie met het optreden van complicaties (zoals hartinfarct, longembolie, nierfalen, sepsis en beroerte) tijdens de ziekenhuisopname is gerelateerd aan een beperking in het dagelijks functioneren tot zes maanden na de operatie. Dit is vooral voor patiënten erg belangrijk aangezien het doel van de operatie is om van ziekte te genezen en/of van belastende symptomen af te komen om de kwaliteit van leven te verbeteren. Voor dit onderzoek hebben we 653 patiënten geselecteerd van 50 jaar en ouder die een geplande niet-hart gerelateerde operatie hebben ondergaan in het UMC Utrecht en University 
Health Network Toronto, Canada. Op basis van een verhoogd troponine en het optreden van complicaties, hebben we hebben patiënten in vijf groepen verdeeld:

1) patiënten met een normale troponine waarde zonder complicaties

2) patiënten met alleen een verhoogd troponine maar geen complicatie

3) patiënten met een hartinfarct en dus een verhoogd troponine

4) patiënten met een verhoogd troponine met een complicatie (geen hartinfarct)

5) patiënten zonder een verhoogd troponine met een complicatie (geen hartinfarct)

In totaal werd in 125 (19\%) van de patiënten een verhoogd troponine gevonden en 168 (26\%) ontwikkelde een beperking in het dagelijks functioneren zes maanden na de operatie. We waren niet in staat om verschillen in beperking in het dagelijks functioneren tussen de groepen aan te tonen. Echter vonden we wel een klinisch relevant verschil in de beperking in het dagelijks functioneren bij patiënten met een hartinfarct (groep 3) en patiënten met een verhoogd troponine met een complicatie (groep 4) ten opzichte van patiënten zonder complicaties (groep 1). Vroege herkenning en behandeling van complicaties (zowel hart- als niet hart gerelateerd) zou kunnen bijdragen in het voorkomen van beperking in het dagelijks functioneren 6 maanden na de operatie.

In conclusie, dit proefschrift focust zich op verschillende methodologisch complexe aspecten van onderzoek in de perioperatieve periode. Toekomstig onderzoek is nodig om de grote hoeveelheid aan meetpunten binnen eenzelfde patiënt beter samen te kunnen vatten voor zowel onderzoeksdoeleinden als voor gebruik binnen de patiëntenzorg. Hierbij zou gedacht kunnen worden aan dynamische voorspellingen, die bijvoorbeeld over de tijd voorspellingen kunnen optimaliseren door steeds nieuwe meetwaarden aan de voorspelling toe te voegen. Daarbij is belangrijk dat er overeenstemming komt in welke standaard methodologieën er gebuikt zouden moeten worden om onderzoeken beter met elkaar te kunnen vergelijken. Daarnaast zou er meer onderzoek plaats kunnen vinden in de selectie van hoog risico patiënten om de zorg voor deze patiënten te optimaliseren. Dit geldt voor zowel tijdens de ziekenhuisopname en de periode van herstel daarna om beperking het dagelijks functioneren zo veel mogelijk te kunnen voorkomen. 


\section{LIST OF PUBLICATIONS}

1. Vernooij LM, van Klei WA, Machina M, Pasma W, Beattie WS, Peelen LM. Different methods of modelling intraoperative hypotension and their association with postoperative complications in patients undergoing non-cardiac surgery. Br J Anaesth. 2018;120(5):10801089. doi:10.1016/j.bja.2018.01.033

2. Vernooij LM, Damen JAAG, van Klei WA, Moons K, Peelen LM. The added value of different biomarkers to the Revised Cardiac Risk Index to predict major adverse cardiac events and all-cause mortality after noncardiac surgery. Cochrane Database of Systematic Reviews 2018, Issue 10. Art. No.: CD013139. DOI: 10.1002/14651858.CD013139.

3. Vernooij LM, van Klei WA, Moons KGM, van Waes JA, Peelen LM. Methods to express intraoperative hypotension exposure in the anaesthesia literature. $\mathrm{Br} J$ Anaesth. 2020;124(3):e35-e37. doi:10.1016/j.bja.2019.12.004

4. Akkermans A, Vernooij LM, van Klei WA, van Waes JA. Postoperative visits by dedicated anesthesiologists in patients with elevated troponin: a retrospective cohort study evaluating postoperative care utility and early detection of complications. Perioper Med (Lond). 2020;9:22. Published 2020 Jul 16. doi:10.1186/s13741-020-00152-6

5. Vernooij LM, Moons KGM, van Klei WA, Takada T, van Waes JAR, Damen JAAG. The added value of biomarkers to the Revised Cardiac Risk Index to preoperatively predict major adverse cardiac events in patients who undergo non-cardiac surgery: a systematic review. In preparation.

6. Vernooij LM, van Waes JAR, van Klei WA, Peelen LM, Beattie WS. Association between postoperative myocardial injury phenotypes and disability-free survival in patients undergoing non-cardiac surgery. In preparation. 


\section{DANKWOORD}

Het zit erop. Na iets meer dan drie jaar hard werken is het schrijven van het dankwoord het laatste onderdeel van mijn promotie, op mijn verdediging en de feestelijkheden die volgen na dan. Hoewel het op dit moment bijzondere en onzekere tijden zijn, ben ik voornamelijk erg dankbaar. Dankbaar voor de mensen om mij heen en de kansen die ik de afgelopen periode heb gekregen en hopelijk nog meer zal gaan krijgen. Ik had mijn promotietraject dan ook niet met succes af kunnen ronden zonder de steun van een hoop collega's, vrienden en familie. Een aantal zou ik graag in het bijzonder bedanken.

Beste Wilton, dank voor al het vertrouwen dat je mij de afgelopen jaren hebt gegeven. Dit begon eigenlijk al voor mijn promotietraject toen ik deel van mijn epidemiologiestage heb mogen doen in Toronto bij prof. Beattie. Het was een voorrecht dat ik de kans kreeg om mijn masterscriptie uit te kunnen breiden tot een zelf-samengesteld promotietraject. Wanneer het mij af en toe te heet onder de voeten werd, zorgde jouw rust ervoor dat ik snel weer de juiste richting op ging. En ook zelfs na mijn promotietraject kreeg ik het vertrouwen om door te gaan als epidemioloog binnen de afdeling Vitale Functies. Ik ben enorm dankbaar.

Beste Carl, dank voor alle inspirerende jaren dat ik onderdeel mocht uitmaken van de Methoden groep binnen het Julius Centrum. Het bijzonder hoge kennisniveau binnen deze saamhorige groep stimuleerde mij om 'on top of my game' te blijven. Bij onze overleggen hield je mij met beide benen op de grond en nam je een stapje terug om weer naar de kern van het onderzoek te gaan. Dat hielp mij om de focus te bewaren. Dank dat ik van je heb mogen leren om een betere onderzoeker te kunnen worden.

Beste Judith, wat ben ik ontzettend blij dat je onderdeel bent geworden van mijn promotieteam. Naast dat ik ons overleg altijd erg gezellig vond, kon ik altijd laagdrempelig bij je terecht met vragen over logistieke zaken rondom de TEAMS studie en 'dokters'-vragen vanuit de kliniek. Je straalt rust uit, bent praktisch ingesteld en zoekt naar de meest efficiënte oplossingen. Ik hoop dat ik dit zelf ook over kan brengen op de promovendi die ik zal bijstaan in hun onderzoek. Ik kijk uit naar alle volgende onderzoeken die we samen aan kunnen pakken. Dank dat je mijn promotietraject zo'n succes heb laten worden!

Lieve Linda, ik kan niet in woorden omschrijven hoe dankbaar ik ben dat ik bij jou mijn onderzoeksstage van de master Epidemiologie mocht doen. Je hebt mij niet alleen doen groeien als wetenschapper, maar ook zeker als mens. Zonder jouw wijsheid binnen de Epidemiologie maar ook zeker in het leven, had ik dit eindresultaat niet kunnen bereiken en was ik niet zo trots geweest op dit proefschrift. Je nam mij in bescherming vooral tegen mijzelf wanneer ik het nodig had, maar gaf ook het zetje op het juist moment om te kunnen 'shinen'. Je zorgt ervoor dat ik net dat stapje extra zet om mijn doel te kunnen behalen. Dat waardeer ik enorm. Dank daarvoor! 
Dear Scott, thank you for the opportunity to work at your research group. Your ideas and experience inspire me to become a better researcher. I'd love to make some more apple pie when you come over for a visit (or give you the recipe).

Beste Martine, Mathilde, Nikki, Tessa, Lynn, Matthijs, Esther, Mariel, Corien, Kirsten, Ivo, Diana, Emma, Wietze, Meri, Marlies, Ilse, Simone en andere onderzoekers van Vitale Functies, dank voor jullie gezelligheid en het luisterend oor bij de moeilijke maar ook zeker bij de mooie momenten. Wietze en Jacqueline, bedankt voor het datamanagement van de TEAMS studie en het maken van de datasets voor de andere onderzoeksprojecten. Dank Frouke en Annemarie voor de fijne samenwerking binnen onze eigen onderzoeksprojecten. Jullie zijn allen voorlopig nog niet van mij af ;-)

Beste Ada, Gea, Joanna en José van het trialbureau, ik wil jullie ontzettend bedanken voor jullie inzet binnen de TEAMS studie. Zonder jullie input en doorzettingsvermogen was dit onderzoek in dit tempo niet mogelijk geweest. Jullie zijn toppers!

Beste collega's van het Julius Centrum STR 6.101 en MvG 5.11, dank voor de adviezen rondom de opzet van een nieuwe studie, de hulp rondom het kraken van de R-code, maar vooral de gezelligheid onder het genot van een hoop koffies van het Micafé. Beste collega's van het epimethoden team, dank voor de gezellige uitjes, de vele gebakken taarten tijdens de weekstart en de inspirerende methodologische praatjes. Lieve Anneke, wat fijn dat jij samen met mij de uitdaging aanging van het schrijven van een Cochrane review. Zonder jouw expertise en precisie had het niet in zo'n ver stadium kunnen zijn. Ik kijk nog steeds uit naar onze overleggen en vind het ontzettend fijn dat ik ook bij carrière gerelateerde zaken bij je terecht kan. Ik hoop op nog een hoop productieve en gezellige samenwerkingen.

Dank aan mijn collega's van het Antonius Ziekenhuis Nieuwegein om mij de ruimte te geven om mijn proefschrift af te kunnen ronden. Lisa, Britta, Rosa, Ellen, Peter, Heleen en Eric, ik ben ontzettend blij dat ik deel mag uitmaken van jullie onderzoeksgroep.

Lieve Karen, sinds het moment dat wij elkaar al meer dan tien jaar geleden ontmoetten ben jij een van mijn beste vriendinnen. Je bent altijd vrolijk, bent de meest sociale persoon die ik ken en gaat uitdagingen niet de weg. Dat bewonder ik aan jou. Ik kijk uit naar de vele (wandel)vakanties en gezellige etentjes die nog zullen gaan komen. Fijn dat je aan mijn zijde wilt staan als paranimf! Lieve Marieke, waar we een aantal jaar geleden nog vaak samen op stap gingen, gaan we nu liever samen op het terras zitten voor een kopje koffie. Ik waardeer jouw eerlijkheid en zorgzaamheid. Dank dat ookjij mijn paranimf wilt zijn. 
Lieve Lisa en Renée, het is alweer meer dan vijf jaar geleden dat we samen aan de master Epidemiologie begonnen. Naast dat we elkaar nog regelmatig om epi-advies vragen, errors in $\mathrm{R}$ overwinnen en ik stiekem met jullie het hardst om (Ryan Gosling) statistiekgrappen kan lachen, heb ik onze breiavonden, etentjes en tennispotjes erg gewaardeerd. Ik hoop dat er nog vele zullen volgen! Lieve Yvonne, jij bent mijn oudste vriendin en dat gaat niemand meer in kunnen halen. Naast dat we zo veel op elkaar lijken, zijn we ook heel verschillend. Dank dat ik bij jou zo mijzelf kan zijn.

Lieve Wim, Ella, Petra en Eric, dank dat jullie mij vijf jaar geleden met veel warmte in jullie familie hebben opgenomen. De etentjes, spontane visites, leuke kaartjes via de post en familieweekenden zijn altijd erg gezellig (hoewel die gekke regels van het sjoelen en yahtzee mij nog steeds niet lekker zitten;-)). Tot de volgende!

Lieve oma, ook al kom ik niet zo vaak langs, dat betekent niet dat ik niet vaak aan jou (en aan opa) denk. Ik hoop dat ik ooit net zo wijs en sterk oud mag worden zoals jij dat nu doet.

Lieve papa en mama, dank dat jullie altijd voor mij klaar staan, mij steunen ook als het even niet zo makkelijk gaat en onvoorwaardelijk trots op mij zijn. Ik zou de woensdagen samen niet meer willen missen. Tim, Koen en Marleen, wat is het fijn om heerlijk bij elkaar op de bank te ploffen om een potje voetbal te kijken met een biertje in de hand of gezellig met elkaar te BBQen. Ik geniet van die momenten dat het allemaal even niet zo serieus is. Dank dat jullie altijd voor mij klaarstaan!

Lieve Robin, op het moment van schrijven zijn we alweer vijf jaar samen en ik zou mij geen dag meer in willen denken zonder jou. Jouw vertrouwen in mij, de steun die je mij geeft en het geduld dat je voor mij hebt hebben geleid tot een hoop persoonlijke overwinningen, waaronder de afronden van dit proefschrift. Samen gaan we de volgende uitdaging aan. Ik houd van je! 


\section{ABOUT THE AUTHOR}

Lisette Vernooij was born on 23rd May 1991 in Odijk. She completed secondary school at De Breul in Zeist and started her bachelor in Biomedical Sciences at the University Utrecht in 2009. She completed her bachelor thesis at the department of Reproductive Medicine and Gynaecology at the UMC Utrecht on the influence of early ovarian hyperresponse on embryo quality and ongoing pregnancies in pre-implantation genetic diagnoses (PGD) patients. She gained interest in research on a healthcare and patient level while working as a project assistant at Mediquest, an independent company performing research on the quality of health care in the Netherlands. In 2015, she began her master Epidemiology at the University Utrecht and

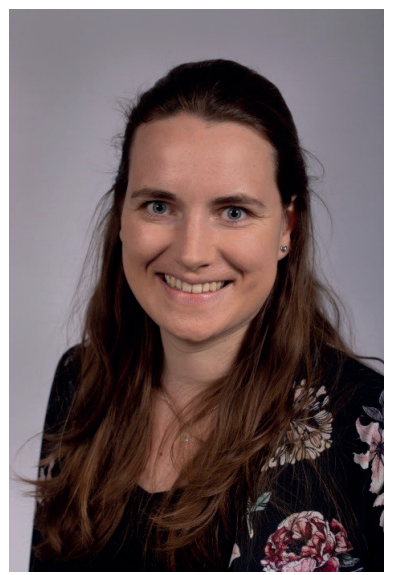
conducted her 13-month internship at the department of Anesthesiology under supervision of dr. Linda Peelen and prof. Wilton van Klei. Her master thesis was on different methods to model intraoperative hypotension and its association with postoperative complications in patients undergoing non-cardiac surgery (Chapter 3 of this thesis). She performed 3 months of this internship at the University Health Network Toronto in Canada under supervision of prof. W. Scott Beattie. In addition, she did a 3 month internship at GlaxoSmithKline in Zeist on the effectivity and safety of Benlysta (belimumab) for the treatment of patients with systemic lupus erythematosus (SLE) under supervision of dr. Stefan Vegter.

In 2016, she was able to extend her research activities during her master thesis into a PhD trajectory on methods in the perioperative period under supervision of prof. Wilton van Klei, prof. Carl Moons, dr. Linda Peelen and dr. Judith van Waes. After finishing her thesis, she started working as an epidemiologist at the department of Anesthesiology at the UMC Utrecht and the St. Antonius Hospital in Nieuwegein. 
